\title{
Rh(III)-Catalyzed [5+1] Annulation of Indole-enaminones with Diazo Compounds to Form Highly Functionalized Carbazoles
}

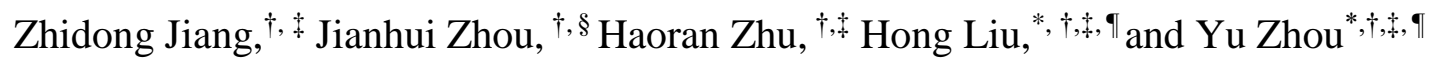

${ }^{\dagger}$ State Key Laboratory of Drug Research, Shanghai Institute of Materia Medica,

Chinese Academy of Sciences, Shanghai 201203, China

\$University of Chinese Academy of Sciences, Beijing 100049, China.

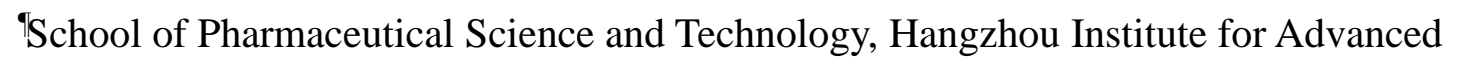
Study, University of Chinese Academy of Sciences, Hangzhou 310024, China

${ }^{\S}$ College of Pharmacy, Nanjing University of Chinese Medicine, Nanjing 210023, China. 


\section{Table of Contents}

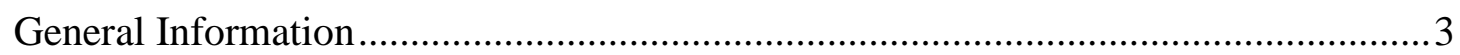

General procedure for synthesis of indole-enaminones and their spectral data..............

General procedure for the synthesis of products and their spectral data .....................12

General methods for mechanism study and their spectral data................................27

General methods for applications and their spectral data .......................................2

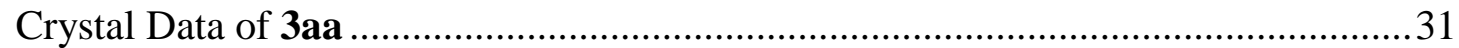

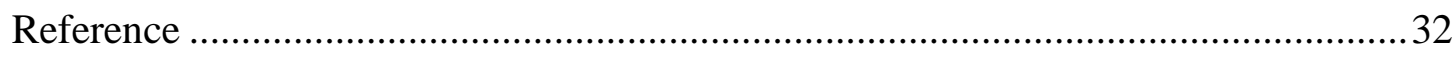

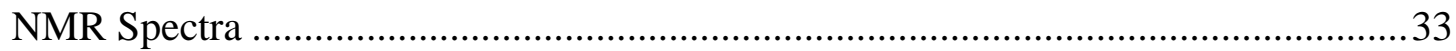




\section{General Information}

Unless otherwise specified, commercially available reagents were purchased from commercial sources (J\&K, Sigma-Aldrich, Adamas-beta, Energy, Bidepharm, Accela, etc.), and used without further purification. Analytical thin layer chromatography (TLC) was performed on HSGF 254 (0.15-0.2 mm thickness), visualized by irradiation with UV light $(254 \mathrm{~nm})$. Column chromatography was performed on silica gel FCP $200-400$ or 300-400 using ethyl acetate (EA)/petroleum ether (PE). All products were characterized by their NMR and HRMS spectra. ${ }^{1} \mathrm{H}$ and ${ }^{13} \mathrm{C}$ NMR spectra were recorded on a 500 , or $600 \mathrm{MHz}$ instrument. The chemical shifts were reported in parts per million (ppm, $\delta$ ) downfield from tetramethylsilane (TMS). Proton coupling patterns were described as singlet (s), doublet (d), triplet (t), quartet (q), multiplet (m), doublet of doublets (dd), and broad (br). High-resolution mass spectra (HRMS) were measured on a Micromass Ultra Q-TOF spectrometer.

\section{General procedure for synthesis of indole-enaminones and their}

\section{spectral data}

\section{Preparation of 1a-1r}

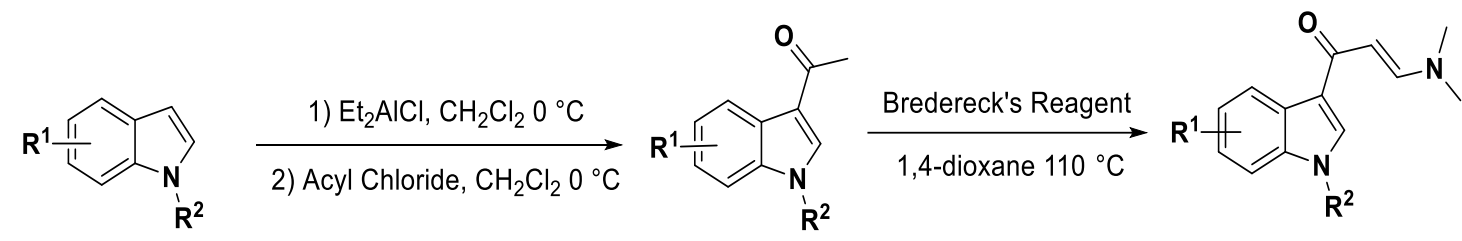

A

B

General procedure of acylation of indoles at the 3-position (B) ${ }^{1}$<smiles>[R]n1ccc2c[R1]#ccc21</smiles>

A

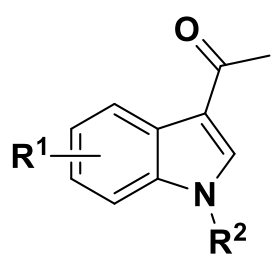

B

To a $\mathrm{CH}_{2} \mathrm{Cl}_{2}$ solution $(13 \mathrm{~mL})$ of indole $(\mathrm{A}, 3 \mathrm{mmol})$ was added $2.26 \mathrm{~mL}(1.5$ 
equiv) of $\mathrm{Et} 2 \mathrm{AlCl}(2 \mathrm{~mol} / \mathrm{L}$ in hexane $)$ dropwise at $0{ }^{\circ} \mathrm{C}$. The mixture was stirred at $0{ }^{\circ} \mathrm{C}$ for $30 \mathrm{~min}$. Then $\mathrm{CH}_{2} \mathrm{Cl}_{2}$ solution $(10 \mathrm{~mL})$ of acetyl chloride (1.5 equiv) was added dropwise at $0{ }^{\circ} \mathrm{C}$. The resulting solution was stirred at same temperature for an appropriate of time (2-4 hrs). After completion of the reaction it was quenched with aqueous buffer ( $\mathrm{pH}$ 7). After usual workup, the crude product was purified by either trituration or flash column chromatography to provide the 3-acetylindole, B (60-94\%yield).

\section{General procedure of substrates $(1)^{2}$}

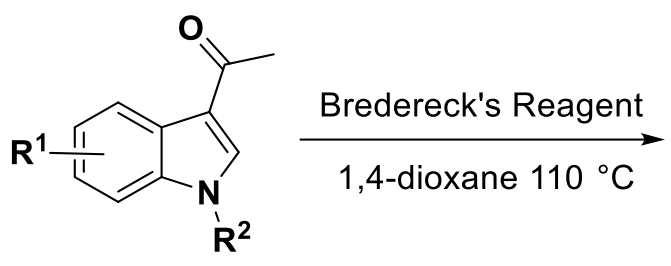

B

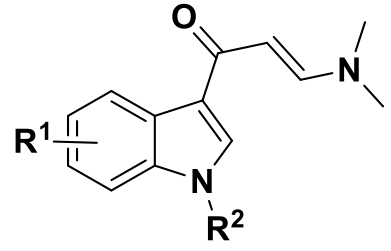

1

To a solution of 3-Acetylindole (B, $2 \mathrm{mmol})$ in 1,4-dioxane $(20 \mathrm{~mL})$ was added Bredereck's reagent $\left(3\right.$ equiv). The reaction mixture was stirred at $110^{\circ} \mathrm{C}$ in an oil bath for $12 \mathrm{~h}$. After that, the solvent was removed under reduced pressure and the residue was washed by EA, then filtered and collect solids to provide product 1(80-90\%yield).

\section{Preparation of $2 \mathrm{a}-2 \mathrm{f}$}

To a solution of ketonic ester or 1,3 diketone (5 mmol) in $\mathrm{CH}_{3} \mathrm{CN}, 6 \mathrm{mmol} \mathrm{TsN}_{3}$ was added. Then the reaction mixture was cooled to $0{ }^{\circ} \mathrm{C}$, and a solution of TEA (6 mmol) in $\mathrm{CH}_{3} \mathrm{CN}$ was added dropwise. Next, the reaction temperature was raised to room temperature. After stirring for $5 \mathrm{~h}$, the residue was extracted with ethyl acetate for 3 times. The combined organic layers were washed with water and brine sequentially, dried over $\mathrm{Na}_{2} \mathrm{SO}_{4}$, filtered, and concentrated. The crude product was purified by flash chromatography on silica gel to afford the corresponding product in $70-90 \%$ yield. 
Characterization of substrates (1a-1r)

(E)-3-(Dimethylamino)-1-(1H-indol-3-yl)prop-2-en-1-one (1a)<smiles>CN(C)/C=C/C(=O)c1c[nH]c2ccccc12</smiles>

yellow solid (300 mg, 70\% overall yield), mp 239-241 ${ }^{\circ} \mathrm{C}$; ${ }^{1} \mathbf{H}$ NMR (600 MHz, DMSO-d6) $\delta 8.21(\mathrm{~d}, J=7.5 \mathrm{~Hz}, 1 \mathrm{H}), 8.12(\mathrm{~s}, 1 \mathrm{H}), 7.48$ (d, $J=12.5 \mathrm{~Hz}, 1 \mathrm{H}), 7.39$ (d, $J=7.8 \mathrm{~Hz}, 1 \mathrm{H}), 7.08-6.95(\mathrm{~m}, 2 \mathrm{H}), 5.77(\mathrm{~d}, J=12.5 \mathrm{~Hz}, 1 \mathrm{H}), 2.93(\mathrm{~s}, 6 \mathrm{H}) .{ }^{13} \mathrm{C}$ NMR (150 MHz, DMSO-d6) $\delta$ 183.8, 150.7, 140.7, 135.3, 127.8, 121.8, 121.0, 120.0, 117.8, 113.7, 94.5, 38.7, 25.7; HRMS m/z $[\mathrm{M}+\mathrm{H}]^{+}$calculated for $\mathrm{C}_{13} \mathrm{H}_{15} \mathrm{~N}_{2} \mathrm{O}$ : 215.1179, found: 215.1173 .

(E)-3-(Dimethylamino)-1-(5-methyl-1H-indol-3-yl)prop-2-en-1-one (1b)<smiles>Cc1ccc2[nH]cc(C(=O)/C=C/N(C)C)c2c1</smiles>

yellow solid (370 mg, 81\% overall yield), mp 210-212 ${ }^{\circ} \mathrm{C} ;{ }^{\mathbf{1}} \mathbf{H}$ NMR (500 MHz, DMSO-d6) $\delta 11.47(\mathrm{~s}, 1 \mathrm{H}), 8.10(\mathrm{~d}, \mathrm{~J}=3.5 \mathrm{~Hz}, 2 \mathrm{H}), 7.52(\mathrm{~d}, \mathrm{~J}=12.5 \mathrm{~Hz}, 1 \mathrm{H}), 7.29$ $(\mathrm{d}, \mathrm{J}=8.2 \mathrm{~Hz}, 1 \mathrm{H}), 6.96(\mathrm{~d}, \mathrm{~J}=8.2 \mathrm{~Hz}, 1 \mathrm{H}), 5.76(\mathrm{~d}, \mathrm{~J}=12.5 \mathrm{~Hz}, 1 \mathrm{H}), 2.96(\mathrm{~s}, 6 \mathrm{H})$, 2.40 (s, 3H). ${ }^{13}$ C NMR (125 MHz, DMSO-d6) $\delta$ 185.5, 152.7, 136.8, 132.1, 131.0, 128.3, 125.3, 123.5, 119.5, 113.2, 95.4, 23.3; HRMS m/z $[\mathrm{M}+\mathrm{H}]^{+}$calculated for $\mathrm{C}_{14} \mathrm{H}_{17} \mathrm{~N}_{2} \mathrm{O}: 229.1335$, found: 229.1334 .

methyl (E)-3-(3-(Dimethylamino)acryloyl)-1H-indole-5-carboxylate (1c)<smiles>COC(=O)c1ccc2[nH]cc(C(=O)/C=C/N(C)C)c2c1</smiles>

yellow solid (408 mg, 75\% overall yield), mp 230-232 ${ }^{\circ} \mathrm{C} ;{ }^{\mathbf{1}} \mathbf{H}$ NMR (500 MHz, DMSO-d6) $\delta 8.79(\mathrm{~s}, 1 \mathrm{H}), 8.08(\mathrm{~s}, 1 \mathrm{H}), 7.42(\mathrm{~d}, \mathrm{~J}=8.4 \mathrm{~Hz}, 1 \mathrm{H}), 7.31(\mathrm{dd}, \mathrm{J}=20.4$, $10.6 \mathrm{~Hz}, 2 \mathrm{H}), 5.75(\mathrm{~d}, \mathrm{~J}=12.7 \mathrm{~Hz}, 1 \mathrm{H}), 3.79$ (s, 3H), 2.91 (s, 6H). ${ }^{13} \mathrm{C}$ NMR (125 
MHz, DMSO-d6) $\delta 182.8,168.6,152.1,148.6,147.7,129.2,123.2,118.7,117.5$, 117.3, 116.2, 95.0, 51.0; HRMS m/z $[\mathrm{M}+\mathrm{H}]^{+}$calculated for $\mathrm{C}_{15} \mathrm{H}_{17} \mathrm{~N}_{2} \mathrm{O}_{3}: 273.1234$, found: 273.1231 .

(E)-3-(Dimethylamino)-1-(5-fluoro-1H-indol-3-yl)prop-2-en-1-one (1d)<smiles>CN(C)/C=C/C(=O)c1c[nH]c2ccc(F)cc12</smiles>

yellow solid (381 mg, 82\% overall yield), mp 250-252 ${ }^{\circ} \mathrm{C} ;{ }^{1} \mathbf{H}$ NMR (600 MHz, DMSO-d6) $\delta 11.71(\mathrm{~s}, 1 \mathrm{H}), 8.23(\mathrm{~s}, 1 \mathrm{H}), 7.96(\mathrm{dd}, J=10.3,2.6 \mathrm{~Hz}, 1 \mathrm{H}), 7.54$ (d, $J=$ $12.5 \mathrm{~Hz}, 1 \mathrm{H}), 7.41(\mathrm{dd}, J=8.8,4.6 \mathrm{~Hz}, 1 \mathrm{H}), 6.99(\mathrm{td}, J=9.1,2.6 \mathrm{~Hz}, 1 \mathrm{H}), 5.76(\mathrm{~d}, J$ $=12.5 \mathrm{~Hz}, 1 \mathrm{H}), 2.97(\mathrm{~s}, 6 \mathrm{H}) .{ }^{13} \mathrm{C}$ NMR (150 MHz, DMSO-d6) $\delta 183.8,158.4(\mathrm{~d}, \mathrm{~J}=$ $232.9 \mathrm{~Hz}), 151.7,133.6,132.3,127.2(\mathrm{~d}, \mathrm{~J}=11.0 \mathrm{~Hz}), 118.5,113.2(\mathrm{~d}, \mathrm{~J}=9.8 \mathrm{~Hz})$, $110.5(\mathrm{~d}, \mathrm{~J}=26.3 \mathrm{~Hz}), 106.9(\mathrm{~d}, \mathrm{~J}=24.2 \mathrm{~Hz}), 93.5 . \mathbf{H R M S ~ m} / \mathbf{z}[\mathrm{M}+\mathrm{H}]^{+}$calculated for $\mathrm{C}_{13} \mathrm{H}_{14} \mathrm{FN}_{2} \mathrm{O}$ : 233.1085, found: 233.1083.

(E)-1-(5-Chloro-1H-indol-3-yl)-3-(dimethylamino)prop-2-en-1-one (1e)<smiles>CN(C)/C=C/C(=O)c1c[nH]c2ccc(Cl)cc12</smiles>

yellow solid (388 mg, 78\% overall yield), mp 267-269 ${ }^{\circ} \mathrm{C}$; ${ }^{1} \mathbf{H}$ NMR (500 MHz, DMSO-d6) $\delta 11.82(\mathrm{~s}, 1 \mathrm{H}), 8.30(\mathrm{~d}, \mathrm{~J}=2.0 \mathrm{~Hz}, 1 \mathrm{H}), 8.24(\mathrm{~s}, 1 \mathrm{H}), 7.56(\mathrm{~d}, \mathrm{~J}=12.5$ $\mathrm{Hz}, 1 \mathrm{H}), 7.43(\mathrm{~d}, \mathrm{~J}=8.6 \mathrm{~Hz}, 1 \mathrm{H}), 7.15(\mathrm{dd}, \mathrm{J}=8.6,2.1 \mathrm{~Hz}, 1 \mathrm{H}), 5.77(\mathrm{~d}, \mathrm{~J}=12.5 \mathrm{~Hz}$, 1H), 2.98 (s, 6H). ${ }^{13}$ C NMR (125 MHz, DMSO-d6) $\delta$ 186.8, 151.8, 135.4, 132.0, 127.8, 125.7, 122.3, 121.4, 118.1, 113.7, 93.4; HRMS m/z $[\mathrm{M}+\mathrm{H}]^{+}$calculated for $\mathrm{C}_{13} \mathrm{H}_{14} \mathrm{ClN}_{2} \mathrm{O}: 249.0789$, found: 249.0795. 
(E)-1-(5-Bromo-1H-indol-3-yl)-3-(dimethylamino)prop-2-en-1-one (1f)<smiles>CN(C)/C=C/C(=O)c1c[nH]c2ccc(Br)cc12</smiles>

yellow solid (364 mg, 62\% overall yield), mp 268-270 ${ }^{\circ} \mathrm{C} ;{ }^{1} \mathbf{H}$ NMR (500 MHz, DMSO-d6) $\delta 8.14$ (s, 1H), $7.97(\mathrm{~s}, 1 \mathrm{H}), 7.33$ (d, J = 12.7 Hz, 1H), 7.23 (d, J = 8.4 Hz, 1H), $6.83(\mathrm{dd}, \mathrm{J}=8.4,2.2 \mathrm{~Hz}, 1 \mathrm{H}), 5.68(\mathrm{~d}, \mathrm{~J}=12.7 \mathrm{~Hz}, 1 \mathrm{H}), 2.89$ (s, 6H). ${ }^{13} \mathbf{C ~ N M R}$ (125 MHz, DMSO-d6) $\delta$ 183.4, 149.4, 147.9, 147.0, 132.2, 122.6, 120.2, 118.9, 116.2, 111.1, 95.0; HRMS m/z $[\mathrm{M}+\mathrm{H}]^{+}$calculated for $\mathrm{C}_{13} \mathrm{H}_{14} \mathrm{BrN}_{2} \mathrm{O}: 293.0284$, found: 293.0290 .

(E)-3-(Dimethylamino)-1-(5-iodo-1H-indol-3-yl)prop-2-en-1-one (1g)<smiles>CN(C)/C=C/C(=O)c1c[nH]c2ccc(I)cc12</smiles>

yellow solid (497 mg, 73\% overall yield), mp 272-274 ${ }^{\circ} \mathrm{C}$; ${ }^{\mathbf{1}} \mathbf{H}$ NMR (500 MHz, DMSO-d6) $\delta 11.76(\mathrm{~s}, 1 \mathrm{H}), 8.67(\mathrm{~d}, \mathrm{~J}=1.6 \mathrm{~Hz}, 1 \mathrm{H}), 8.17(\mathrm{~s}, 1 \mathrm{H}), 7.55(\mathrm{~d}, \mathrm{~J}=12.5$ $\mathrm{Hz}, 1 \mathrm{H}), 7.41(\mathrm{dd}, \mathrm{J}=8.5,1.7 \mathrm{~Hz}, 1 \mathrm{H}), 7.28(\mathrm{~d}, \mathrm{~J}=8.5 \mathrm{~Hz}, 1 \mathrm{H}), 5.76(\mathrm{~d}, \mathrm{~J}=12.5 \mathrm{~Hz}$, 1H), 2.98 (s, 6H). 13C NMR (125 MHz, DMSO-d6) $\delta$ 183.7, 151.8, 136.0, 131.4, 130.8, 130.3, 129.3, 117.7, 114.6, 93.5, 85.34; HRMS m/z $[\mathrm{M}+\mathrm{H}]^{+}$calculated for $\mathrm{C}_{13} \mathrm{H}_{14} \mathrm{IN}_{2} \mathrm{O}: 341.0145$, found: 341.0142 .

(E)-3-(Dimethylamino)-1-(6-methyl-1H-indol-3-yl)prop-2-en-1-one (1h)<smiles>Cc1ccc2c(C(=O)/C=C/N(C)C)c[nH]c2c1</smiles>

yellow solid (356 mg, 78\% overall yield), mp 210-212 ${ }^{\circ} \mathrm{C}$; ${ }^{1} \mathbf{H}$ NMR (500 MHz, DMSO-d6) $\delta 11.44(\mathrm{~s}, 1 \mathrm{H}), 8.15(\mathrm{~d}, \mathrm{~J}=8.1 \mathrm{~Hz}, 1 \mathrm{H}), 8.06(\mathrm{~s}, 1 \mathrm{H}), 7.52(\mathrm{~d}, \mathrm{~J}=12.5$ $\mathrm{Hz}, 1 \mathrm{H}), 7.20$ (s, 1H), 6.93 (dd, J = 8.1, 1.0 Hz, 1H), 5.76 (d, J = 12.5 Hz, 1H), 2.96 (s, 6H), 2.39 (s, 3H). ${ }^{13}$ C NMR (125 MHz, DMSO-d6) $\delta 185.4,152.8,138.9,132.8$, 
131.5, 125.9, 124.1, 123.4, 119.9, 113.3, 95.3, 23.2; $\mathbf{H R M S ~ m / z ~}[\mathrm{M}+\mathrm{H}]^{+}$calculated for $\mathrm{C}_{14} \mathrm{H}_{17} \mathrm{~N}_{2} \mathrm{O}: 229.1335$, found: 229.1334 .

methyl (E)-3-(3-(Dimethylamino)acryloyl)-1H-indole-6-carboxylate (1i)<smiles>COC(=O)c1ccc2c(C(=O)/C=C/N(C)C)c[nH]c2c1</smiles>

yellow solid (354 mg, 65\% overall yield), mp 235-237 ${ }^{\circ} \mathrm{C} ;{ }^{1} \mathbf{H}$ NMR (500 MHz, DMSO-d6) $\delta 11.97$ (s, 1H), 8.38 (s, 2H), 8.07 (s, 1H), 7.73 (d, J = $7.1 \mathrm{~Hz}, 1 \mathrm{H}), 7.58$ $(\mathrm{d}, \mathrm{J}=11.9 \mathrm{~Hz}, 1 \mathrm{H}), 5.79(\mathrm{~d}, \mathrm{~J}=11.8 \mathrm{~Hz}, 1 \mathrm{H}), 3.87$ (s, 3H), 2.99 (s, 6H). ${ }^{13} \mathbf{C}$ NMR (125 MHz, DMSO-d6) $\delta$ 183.6, 167.5, 152.0, 136.4, 133.8, 130.3, 123.4, 122.1, 121.6, 118.8, 114.0, 93.6, 52.3; HRMS m/z $[\mathrm{M}+\mathrm{H}]^{+}$calculated for $\mathrm{C}_{15} \mathrm{H}_{17} \mathrm{~N}_{2} \mathrm{O}_{3}$ : 273.1234, found: 273.1238 .

(E)-3-(Dimethylamino)-1-(6-fluoro-1H-indol-3-yl)prop-2-en-1-one (1j)<smiles>CN(C)/C=C/C(=O)c1c[nH]c2cc(F)ccc12</smiles>

yellow solid (358 mg, 77\% overall yield), mp 246-248 ${ }^{\circ} \mathrm{C}$; ${ }^{1} \mathbf{H}$ NMR (500 MHz, DMSO-d6) $\delta 11.65$ (s, 1H), $8.28(\mathrm{~s}, 1 \mathrm{H}), 8.16(\mathrm{~s}, 1 \mathrm{H}), 7.55$ (d, J = $12.0 \mathrm{~Hz}, 1 \mathrm{H}), 7.19$ $(\mathrm{d}, \mathrm{J}=8.8 \mathrm{~Hz}, 1 \mathrm{H}), 6.96(\mathrm{~s}, 1 \mathrm{H}), 5.77(\mathrm{~d}, \mathrm{~J}=12.1 \mathrm{~Hz}, 1 \mathrm{H}), 2.97(\mathrm{~s}, 6 \mathrm{H}) .{ }^{13} \mathbf{C}$ NMR (125 MHz, DMSO-d6) $\delta 183.8,159.4$ (d, J = 235.5 Hz), 151.7, 136.9 (d, J = 12.4 Hz), 131.1, 123.4, 123.4, 118.5, 109.3 (d, J = 23.7 Hz), 98.2 (d, J = 25.6 Hz), 93.6; HRMS $\mathbf{m} / \mathbf{z}[\mathrm{M}+\mathrm{H}]^{+}$calculated for $\mathrm{C}_{13} \mathrm{H}_{14} \mathrm{FN}_{2} \mathrm{O}: 233.1085$, found: 233.1084 .

(E)-1-(6-Chloro-1H-indol-3-yl)-3-(dimethylamino)prop-2-en-1-one (1k)<smiles>CN(C)/C=C/C(=O)c1c[nH]c2cc(Cl)ccc12</smiles>

yellow solid (388 mg, 78\% overall yield), mp 259-261 ${ }^{\circ} \mathrm{C} ;{ }^{1} \mathbf{H}$ NMR (500 MHz, 
DMSO-d6) $\delta 11.70(\mathrm{~s}, 1 \mathrm{H}), 8.28(\mathrm{~d}, \mathrm{~J}=8.5 \mathrm{~Hz}, 1 \mathrm{H}), 8.20(\mathrm{~s}, 1 \mathrm{H}), 7.56(\mathrm{~d}, \mathrm{~J}=12.5$ $\mathrm{Hz}, 1 \mathrm{H}), 7.46(\mathrm{~d}, \mathrm{~J}=1.9 \mathrm{~Hz}, 1 \mathrm{H}), 7.12(\mathrm{dd}, \mathrm{J}=8.5,1.9 \mathrm{~Hz}, 1 \mathrm{H}), 5.77$ (d, J = $12.5 \mathrm{~Hz}$, 1H), 2.97 (s, 6H). ${ }^{13}$ C NMR (125 MHz, DMSO-d6) $\delta$ 183.7, 151.8, 137.4, 131.5, 127.0, 125.4, 123.6, 121.3, 118.5, 111.8, 93.5; HRMS m/z $[\mathrm{M}+\mathrm{H}]^{+}$calculated for $\mathrm{C}_{13} \mathrm{H}_{14} \mathrm{ClN}_{2} \mathrm{O}$ : 249.0789 , found: 249.0788 .

(E)-3-(Dimethylamino)-1-(7-methoxy-1H-indol-3-yl)prop-2-en-1-one (11)<smiles>COc1cccc2c(C(=O)/C=C/N(C)C)c[nH]c12</smiles>

yellow solid (283 mg, 58\% overall yield), mp 230-232 ${ }^{\circ} \mathrm{C} ;{ }^{\mathbf{1}} \mathbf{H}$ NMR (500 MHz, DMSO-d6) $\delta 11.71(\mathrm{~s}, 1 \mathrm{H}), 8.05(\mathrm{~s}, 1 \mathrm{H}), 7.87(\mathrm{~d}, \mathrm{~J}=7.9 \mathrm{~Hz}, 1 \mathrm{H}), 7.53(\mathrm{~d}, \mathrm{~J}=12.4$ $\mathrm{Hz}, 1 \mathrm{H}), 7.02$ (t, J = 7.7 Hz, 1H), 6.71 (d, J = 7.5 Hz, 1H), 5.79 (d, J = 12.4 Hz, 1H), 3.92 (s, 3H), 2.96 (s, 6H). ${ }^{13}$ C NMR (125 MHz, DMSO-d6) $\delta 184.05,151.5,146.5$, $130.1,128.2,127.0,121.6,119.1,115.1,103.0,94.0,55.6 ; \mathbf{H R M S ~ m} / \mathbf{z}[\mathrm{M}+\mathrm{H}]^{+}$ calculated for $\mathrm{C}_{14} \mathrm{H}_{17} \mathrm{~N}_{2} \mathrm{O}_{2}: 245.1285$, found: 245.1281 .

(E)-3-(Dimethylamino)-1-(7-fluoro-1H-indol-3-yl)prop-2-en-1-one (1m)<smiles>CN(C)/C=C/C(=O)c1c[nH]c2c(F)cccc12</smiles>

yellow solid (330 mg, 71\% overall yield), mp 252-254 ${ }^{\circ} \mathrm{C}$; ${ }^{1} \mathbf{H}$ NMR (500 MHz, DMSO-d6) $\delta 12.10(\mathrm{~s}, 1 \mathrm{H}), 8.23(\mathrm{~s}, 1 \mathrm{H}), 8.11(\mathrm{~d}, \mathrm{~J}=7.9 \mathrm{~Hz}, 1 \mathrm{H}), 7.57(\mathrm{~d}, \mathrm{~J}=12.5$ $\mathrm{Hz}, 1 \mathrm{H}), 7.07(\mathrm{td}, \mathrm{J}=7.9,5.0 \mathrm{~Hz}, 1 \mathrm{H}), 6.98(\mathrm{dd}, \mathrm{J}=11.4,7.8 \mathrm{~Hz}, 1 \mathrm{H}), 5.81(\mathrm{~d}, \mathrm{~J}=$ $12.5 \mathrm{~Hz}, 1 \mathrm{H}), 2.98$ (s, 6H). ${ }^{13} \mathrm{C}$ NMR (125 MHz, DMSO-d6) $\delta 185.2,153.3,151.0$ (d, $\mathrm{J}=243.4 \mathrm{~Hz}), 132.9,131.9(\mathrm{~d}, \mathrm{~J}=4.9 \mathrm{~Hz}), 126.2(\mathrm{~d}, \mathrm{~J}=12.9 \mathrm{~Hz}), 122.8(\mathrm{~d}, \mathrm{~J}=6.0$ Hz), 120.8, 119.9 (d, J = 3.1 Hz), $108.6(\mathrm{~d}, \mathrm{~J}=15.7 \mathrm{~Hz}), 95.1 . \mathbf{H R M S} \mathbf{m} / \mathbf{z}[\mathrm{M}+\mathrm{H}]^{+}$ calculated for $\mathrm{C}_{13} \mathrm{H}_{14} \mathrm{FN}_{2} \mathrm{O}$ : 233.1085, found: 233.1089 . 
(E)-1-(7-Bromo-1H-indol-3-yl)-3-(dimethylamino)prop-2-en-1-one (1n)<smiles>CN(C)/C=C/C(=O)c1c[nH]c2c(Br)cccc12</smiles>

orange solid (340 mg, 58\% overall yield), mp 260-262 ${ }^{\circ} \mathrm{C} ;{ }^{1} \mathbf{H}$ NMR (500 MHz, DMSO-d6) $\delta 11.80(\mathrm{~s}, 1 \mathrm{H}), 8.32(\mathrm{~d}, \mathrm{~J}=7.7 \mathrm{~Hz}, 1 \mathrm{H}), 8.22(\mathrm{~s}, 1 \mathrm{H}), 7.57$ (d, J = 12.4 Hz, 1H), 7.37 (d, J = 7.6 Hz, 1H), 7.06 (t, J = 7.8 Hz, 1H), 5.83 (d, J = 12.4 Hz, 1H), 2.94 (s, 6H). ${ }^{13}$ C NMR (125 MHz, DMSO-d6) $\delta$ 183.8, 152.0, 135.3, 131.5, 128.4, 125.0, 122.5, 121.9, 119.5, 104.7, 93.6; HRMS $\mathbf{m} / \mathbf{z}[\mathrm{M}+\mathrm{H}]^{+}$calculated for $\mathrm{C}_{13} \mathrm{H}_{14} \mathrm{BrN}_{2} \mathrm{O}: 293.0284$, found: 293.0284 .

(E)-3-(Dimethylamino)-1-(4-methyl-1H-indol-3-yl)prop-2-en-1-one (10)<smiles>Cc1cccc2[nH]cc(C(=O)/C=C/N(C)C)c12</smiles>

yellow solid (315 mg, 69\% overall yield), mp 208-210 ${ }^{\circ} \mathrm{C} ;{ }^{1} \mathbf{H}$ NMR (500 MHz, DMSO-d6) $\delta 11.47$ (s, 1H), $7.88(\mathrm{~s}, 1 \mathrm{H}), 7.50(\mathrm{~d}, \mathrm{~J}=12.5 \mathrm{~Hz}, 1 \mathrm{H}), 7.24$ (d, J = 7.8 $\mathrm{Hz}, 1 \mathrm{H}), 7.03(\mathrm{t}, \mathrm{J}=7.4 \mathrm{~Hz}, 1 \mathrm{H}), 6.84(\mathrm{~d}, \mathrm{~J}=6.7 \mathrm{~Hz}, 1 \mathrm{H}), 5.61(\mathrm{~d}, \mathrm{~J}=12.5 \mathrm{~Hz}, 1 \mathrm{H})$, 2.95 (s, 6H), 2.65 (s, 3H). ${ }^{13}$ C NMR (125 MHz, DMSO-d6) $\delta$ 184.2, 151.6, 136.6, $130.8,129.2,124.1,121.4 \times 2,119.7,108.8,95.4,21.7 ; \mathbf{H R M S ~ m / z ~}[\mathrm{M}+\mathrm{H}]^{+}$ calculated for $\mathrm{C}_{14} \mathrm{H}_{17} \mathrm{~N}_{2} \mathrm{O}: 229.1335$, found: 229.1331 .

(E)-3-(Dimethylamino)-1-(4-fluoro-1H-indol-3-yl)prop-2-en-1-one (1p)<smiles>CN(C)/C=C/C(=O)c1c[nH]c2cccc(F)c12</smiles>

yellow solid (376 mg, 81\% overall yield), mp 238-240 ${ }^{\circ} \mathrm{C} ;{ }^{1} \mathbf{H}$ NMR (600 MHz, DMSO-d6) $\delta 11.76(\mathrm{~s}, 1 \mathrm{H}), 7.89(\mathrm{~s}, 1 \mathrm{H}), 7.44(\mathrm{~d}, \mathrm{~J}=12.5 \mathrm{~Hz}, 1 \mathrm{H}), 7.15$ (d, J = 8.1 $\mathrm{Hz}, 1 \mathrm{H}), 7.01(\mathrm{td}, \mathrm{J}=7.9,4.8 \mathrm{~Hz}, 1 \mathrm{H}), 6.73(\mathrm{dd}, \mathrm{J}=11.7,7.8 \mathrm{~Hz}, 1 \mathrm{H}), 5.65(\mathrm{~d}, \mathrm{~J}=$ $12.5 \mathrm{~Hz}, 1 \mathrm{H}), 2.85$ (s, 6H). ${ }^{13} \mathrm{C}$ NMR (150 MHz, DMSO-d6) $\delta 182.6,156.3$ (d, J = 
$248.9 \mathrm{~Hz}), 152.3,140.2(\mathrm{~d}, \mathrm{~J}=11.7 \mathrm{~Hz}), 131.0,123.0(\mathrm{~d}, \mathrm{~J}=7.9 \mathrm{~Hz}), 119.0(\mathrm{~d}, \mathrm{~J}=5.1$ Hz), 113.7 (d, J = 20.1 Hz), 108.8 (d, J = 3.3 Hz), 106.5 (d, J = 21.6 Hz), 95.2; HRMS $\mathbf{m} / \mathbf{z}[\mathrm{M}+\mathrm{H}]^{+}$calculated for $\mathrm{C}_{13} \mathrm{H}_{14} \mathrm{FN}_{2} \mathrm{O}: 233.1085$, found: 233.1081 .

(E)-1-(5,6-Dimethoxy-1H-indol-3-yl)-3-(dimethylamino)prop-2-en-1-one (1q)<smiles>COc1cc2[nH]cc(C(=O)/C=C/N(C)C)c2cc1OC</smiles>

brown solid (384 mg, 70\% overall yield), mp 242-244 ${ }^{\circ} \mathrm{C} ;{ }^{1} \mathbf{H}$ NMR (600 MHz, DMSO-d6) $\delta 11.24(\mathrm{~s}, 1 \mathrm{H}), 7.86(\mathrm{~d}, \mathrm{~J}=2.6 \mathrm{~Hz}, 1 \mathrm{H}), 7.70(\mathrm{~s}, 1 \mathrm{H}), 7.38(\mathrm{~d}, \mathrm{~J}=12.5$ $\mathrm{Hz}, 1 \mathrm{H}), 6.81(\mathrm{~s}, 1 \mathrm{H}), 5.63(\mathrm{~d}, \mathrm{~J}=12.5 \mathrm{~Hz}, 1 \mathrm{H}), 3.66(\mathrm{~s}, 3 \mathrm{H}), 3.66(\mathrm{~s}, 3 \mathrm{H}), 2.84(\mathrm{~s}$, 6H). ${ }^{13}$ C NMR (150 MHz, DMSO-d6) $\delta$ 184.2, 151.2, 147.1, 145.9, 131.2, 128.8, 119.6, 118.4, 104.5, 95.6, 93.8, 56.2, 56.0; HRMS m/z $[\mathrm{M}+\mathrm{H}]^{+}$calculated for $\mathrm{C}_{15} \mathrm{H}_{19} \mathrm{~N}_{2} \mathrm{O}_{3}: 275.1390$, found: 275.1387.

(E)-1-(5-Bromo-1-methyl-1H-indol-3-yl)-3-(dimethylamino)prop-2-en-1-one (1r)<smiles>CN(C)/C=C/C(=O)c1cn(C)c2ccc(Br)cc12</smiles>

yellow solid (338 mg, 55\% overall yield), mp 250-252 ${ }^{\circ} \mathrm{C} ;{ }^{1} \mathbf{H}$ NMR (500 MHz, DMSO-d6) $\delta 8.46(\mathrm{~d}, \mathrm{~J}=2.0 \mathrm{~Hz}, 1 \mathrm{H}), 8.22(\mathrm{~s}, 1 \mathrm{H}), 7.56(\mathrm{~d}, \mathrm{~J}=12.4 \mathrm{~Hz}, 1 \mathrm{H}), 7.46(\mathrm{~d}$, $\mathrm{J}=8.7 \mathrm{~Hz}, 1 \mathrm{H}), 7.33(\mathrm{dd}, \mathrm{J}=8.7,2.0 \mathrm{~Hz}, 1 \mathrm{H}), 5.70(\mathrm{~d}, \mathrm{~J}=12.5 \mathrm{~Hz}, 1 \mathrm{H}), 3.83(\mathrm{~s}, 3 \mathrm{H})$, 2.98 (s, 6H). ${ }^{13}$ C NMR (125 MHz, DMSO-d6) $\delta$ 183.2, 151.82, 136.3, 135.7, 128.8, 124.9, 124.6, 116.8, 114.2, 112.8, 93.3, 33.54; HRMS m/z $[\mathrm{M}+\mathrm{H}]^{+}$calculated for $\mathrm{C}_{14} \mathrm{H}_{16} \mathrm{BrN}_{2} \mathrm{O}$ : 307.0441, found: 307.0441. 


\section{General procedure for the synthesis of products and their spectral}

\section{data}

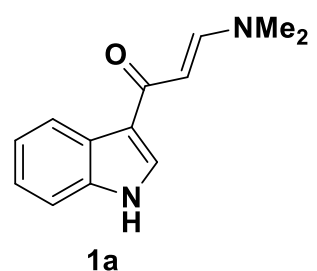

A

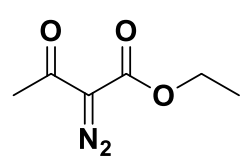

$2 a$

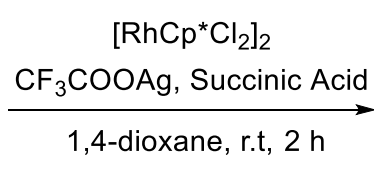

was

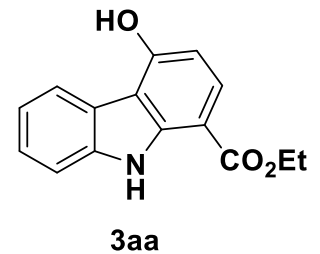

with

(E)-3-(dimethylamino)-1-(1H-indol-3-yl)prop-2-en-1-one (1a, $64.3 \mathrm{mg}, 0.3 \mathrm{mmol})$ and ethyl 2-diazo-3-oxobutanoate (2a, $70.3 \mathrm{mg}, 0.45 \mathrm{mmol}),\left[\mathrm{Cp}^{*} \mathrm{RhCl}_{2}\right]_{2}(9.3 \mathrm{mg}$, $5 \mathrm{~mol} \%), \mathrm{CF}_{3} \mathrm{COOAg}(13.3 \mathrm{mg}, 20 \mathrm{~mol} \%)$ Succinic acid (70.9 mg, $\left.0.6 \mathrm{mmol}\right)$ and 1,4-dioxane $(1 \mathrm{~mL})$. The reaction mixture was stirred at room temperature for $2 \mathrm{~h}$. After that, the solvent was removed under reduced pressure and the residue was purified by silica gel chromatography using PE/EA (10:1) to afford the product 3aa. mp 196-198 ${ }^{\circ} \mathrm{C}$; ${ }^{1}$ H NMR (500 MHz, DMSO-d6) $\delta 11.22$ (s, 1H), 11.06 (s, 1H), 8.18 (d, J = $7.8 \mathrm{~Hz}, 1 \mathrm{H}), 7.87$ (d, J = 8.4 Hz, 1H), 7.70 (d, J = 8.1 $\mathrm{Hz}, 1 \mathrm{H}), 7.40-7.32(\mathrm{~m}, 1 \mathrm{H}), 7.18(\mathrm{t}, \mathrm{J}=7.5 \mathrm{~Hz}, 1 \mathrm{H}), 6.69(\mathrm{~d}, \mathrm{~J}=8.5 \mathrm{~Hz}, 1 \mathrm{H})$, $4.40(\mathrm{q}, \mathrm{J}=7.1 \mathrm{~Hz}, 2 \mathrm{H}), 1.37(\mathrm{t}, \mathrm{J}=7.1 \mathrm{~Hz}, 3 \mathrm{H}) .{ }^{13} \mathbf{C}$ NMR (125 MHz, DMSO-d6) $\delta 165.9,158.5,141.4,139.2,129.4,124.8,121.9,121.4,119.4,111.6$, 111.2, 105.0, 103.4, 59.8, 14.5; HRMS m/z $[\mathrm{M}-\mathrm{H}]^{-}$calculated for $\mathrm{C}_{15} \mathrm{H}_{12} \mathrm{NO}_{3}$ : 254.0823, found: 254.0824 .

\section{Characterization Data of Products}

Ethyl 4-hydroxy-6-methyl-9H-carbazole-1-carboxylate (3ba)<smiles>CCOC(=O)c1ccc(O)c2c1[nH]c1ccc(C)cc12</smiles>

Following by general procedure for the synthesis of $\mathbf{3 b a}$. White solid, $77 \mathrm{mg}$, yield: $95 \%$ (purified by silica gel chromatography using PE/EA 10:1). mp 210-212 ${ }^{\circ} \mathrm{C} ;{ }^{1} \mathbf{H}$ 
NMR (500 MHz, DMSO-d6) $\delta 11.07$ (s, 1H), 10.97 (s, 1H), 7.97 (s, 1H), 7.83 (d, J = $8.4 \mathrm{~Hz}, 1 \mathrm{H}), 7.55(\mathrm{~d}, \mathrm{~J}=8.2 \mathrm{~Hz}, 1 \mathrm{H}), 7.16(\mathrm{~d}, \mathrm{~J}=8.2 \mathrm{~Hz}, 1 \mathrm{H}), 6.64(\mathrm{~d}, \mathrm{~J}=8.5 \mathrm{~Hz}$, 1H), 4.37 (q, J = 7.1 Hz, 2H), 2.44 (s, 3H), 1.35 (t, J = 7.1 Hz, 3H). ${ }^{13}$ C NMR (125 MHz, DMSO-d6) $\delta 165.9,158.5,141.6,137.4,129.3,128.0,126.1,121.9,121.6$, 111.3, 111.1, 104.8, 103.4, 59.8, 21.2, 14.5; HRMS m/z [M - H] $]^{-}$calculated for $\mathrm{C}_{16} \mathrm{H}_{14} \mathrm{NO}_{3}: 268.0979$, found: 268.0976.

\section{1-Ethyl 6-methyl 4-hydroxy-9H-carbazole-1,6-dicarboxylate (3ca)}<smiles>CCOC(=O)c1ccc(O)c2c1[nH]c1ccc(C(=O)OC)cc12</smiles>

Following by general procedure for the synthesis of 3ca. White solid, $38 \mathrm{mg}$, yield: $40 \%$ (purified by silica gel chromatography using PE/EA 10:1). mp 212-214 ${ }^{\circ} \mathrm{C} ;{ }^{1} \mathbf{H}$ NMR (600 MHz, DMSO-d6) $\delta 11.61$ (s, 1H), 11.26 (s, 1H), 8.78 (s, 1H), 7.99 - 7.96 (m, 1H), 7.89 (d, J = 8.5 Hz, 1H), $7.73(\mathrm{~d}, \mathrm{~J}=8.5 \mathrm{~Hz}, 1 \mathrm{H}), 6.73(\mathrm{~d}, \mathrm{~J}=8.5 \mathrm{~Hz}, 1 \mathrm{H}), 4.38$ (q, J = $7.1 \mathrm{~Hz}, 2 \mathrm{H}), 3.85(\mathrm{~s}, 3 \mathrm{H}), 1.35$ (t, J = 7.1 Hz, 3H). ${ }^{13} \mathrm{C}$ NMR (150 MHz, DMSO-d6) $\delta 167.4,166.1,159.0,142.7,142.5,130.7,126.6,124.3,121.7,121.2$, 112.0, 111.6, 106.3, 104.5, 60.5, 52.3, 14.9; HRMS m/z $[\mathrm{M}-\mathrm{H}]^{-}$calculated for $\mathrm{C}_{17} \mathrm{H}_{14} \mathrm{NO}_{5}$ : 312.0877 , found: 312.0880 .

\section{Ethyl 6-fluoro-4-hydroxy-9H-carbazole-1-carboxylate (3da)}<smiles>CCOC(=O)c1ccc(O)c2c1[nH]c1ccc(F)cc12</smiles>

Following by general procedure for the synthesis of 3da. White solid, $78 \mathrm{mg}$, yield: 95\% (purified by silica gel chromatography using PE/EA 10:1). mp 186-188 ${ }^{\circ} \mathrm{C} ;{ }^{1} \mathbf{H}$ NMR (500 MHz, DMSO-d6) $\delta 11.28$ (s, 1H), $11.18(\mathrm{~s}, 1 \mathrm{H}), 7.88$ (d, J = 8.4 Hz, 1H), $7.82(\mathrm{dd}, \mathrm{J}=9.3,2.6 \mathrm{~Hz}, 1 \mathrm{H}), 7.67(\mathrm{dd}, \mathrm{J}=8.8,4.6 \mathrm{~Hz}, 1 \mathrm{H}), 7.21(\mathrm{td}, \mathrm{J}=9.4,2.7 \mathrm{~Hz}$, $1 \mathrm{H}), 6.65(\mathrm{~d}, \mathrm{~J}=8.5 \mathrm{~Hz}, 1 \mathrm{H}), 4.38(\mathrm{q}, \mathrm{J}=7.1 \mathrm{~Hz}, 2 \mathrm{H}), 1.35(\mathrm{t}, \mathrm{J}=7.1 \mathrm{~Hz}, 3 \mathrm{H}) .{ }^{13} \mathrm{C}$ NMR (125 MHz, DMSO-d6) $\delta$ 165.7, 158.6, 156.8 (d, J = 232.6 Hz), 142.3, 135.7, 
130.2, $121.8(\mathrm{~d}, \mathrm{~J}=10.5 \mathrm{~Hz}), 112.6(\mathrm{~m}), 112.4,111.1(\mathrm{~d}, \mathrm{~J}=3.8 \mathrm{~Hz}), 107.0(\mathrm{~d}, \mathrm{~J}=$ 24.4 Hz), 104.8, 103.6, 59.9, 14.5. HRMS m/z [M - H] $]^{-}$calculated for $\mathrm{C}_{15} \mathrm{H}_{11} \mathrm{FNO}_{3}$ : 272.0728, found: 272.0722 .

Ethyl 6-chloro-4-hydroxy-9H-carbazole-1-carboxylate (3ea)<smiles>CCOC(=O)c1ccc(O)c2c1[nH]c1ccc(Cl)cc12</smiles>

Following by general procedure for the synthesis of 3ea. White solid, 77 mg, yield: 89\% (purified by silica gel chromatography using PE/EA 10:1). mp 196-198 ${ }^{\circ} \mathrm{C}$; ${ }^{1} \mathbf{H}$ NMR (500 MHz, DMSO-d6) $\delta 11.38(\mathrm{~s}, 1 \mathrm{H}), 11.24(\mathrm{~s}, 1 \mathrm{H}), 8.09$ (d, J = $2.2 \mathrm{~Hz}, 1 \mathrm{H}), 7.88$ $(\mathrm{d}, \mathrm{J}=8.5 \mathrm{~Hz}, 1 \mathrm{H}), 7.68(\mathrm{~d}, \mathrm{~J}=8.6 \mathrm{~Hz}, 1 \mathrm{H}), 7.37(\mathrm{dd}, \mathrm{J}=8.6,2.2 \mathrm{~Hz}, 1 \mathrm{H}), 6.68(\mathrm{~d}, \mathrm{~J}$ $=8.5 \mathrm{~Hz}, 1 \mathrm{H}), 4.38(\mathrm{q}, \mathrm{J}=7.1 \mathrm{~Hz}, 2 \mathrm{H}), 1.35(\mathrm{t}, \mathrm{J}=7.1 \mathrm{~Hz}, 3 \mathrm{H}) .{ }^{\mathbf{1 3}} \mathbf{C}$ NMR (125 MHz, DMSO-d6) $\delta 165.7,158.6,141.9,137.8,130.3,124.7,123.6,122.6,120.9,113.2$, 110.5, 105.2, 103.7, 60.0, 14.5; HRMS m/z $[\mathrm{M}-\mathrm{H}]^{-}$calculated for $\mathrm{C}_{15} \mathrm{H}_{11} \mathrm{ClNO}_{3}$ : 288.0433, found: 288.0431 .

Ethyl 6-bromo-4-hydroxy-9H-carbazole-1-carboxylate (3fa)<smiles>CCOC(=O)c1ccc(O)c2c1[nH]c1ccc(Br)cc12</smiles>

Following by general procedure for the synthesis of $\mathbf{3 f a}$. White solid, $89 \mathrm{mg}$, yield: $89 \%$ (purified by silica gel chromatography using PE/EA 10:1). mp 212-214 ${ }^{\circ} \mathrm{C} ;{ }^{1} \mathbf{H}$ NMR (500 MHz, DMSO-d6) $\delta 11.41(\mathrm{~s}, 1 \mathrm{H}), 11.25$ (s, 1H), 8.26 (d, J = 2.0 Hz, 1H), 7.91 $(\mathrm{d}, \mathrm{J}=8.5 \mathrm{~Hz}, 1 \mathrm{H}), 7.66(\mathrm{~d}, \mathrm{~J}=8.6 \mathrm{~Hz}, 1 \mathrm{H}), 7.51(\mathrm{dd}, \mathrm{J}=8.6,2.1 \mathrm{~Hz}, 1 \mathrm{H}), 6.71(\mathrm{~d}, \mathrm{~J}$ $=8.5 \mathrm{~Hz}, 1 \mathrm{H}), 4.41(\mathrm{q}, \mathrm{J}=7.1 \mathrm{~Hz}, 2 \mathrm{H}), 1.38(\mathrm{t}, \mathrm{J}=7.1 \mathrm{~Hz}, 3 \mathrm{H}) .{ }^{13} \mathrm{C}$ NMR (125 MHz, DMSO-d6) $\delta 166.1,159.1,142.2,138.5,130.8,127.8,124.3,123.7,114.1,111.9$, 110.8, 105.7, 104.2, 60.4, 14.9; HRMS m/z [M - H] calculated for $\mathrm{C}_{15} \mathrm{H}_{11} \mathrm{BrNO}_{3}$ : 331.9928, found: 331.9922 . 
Ethyl 4-hydroxy-6-iodo-9H-carbazole-1-carboxylate (3ga)<smiles>CCOC(=O)c1ccc(O)c2c1[nH]c1ccc(I)cc12</smiles>

Following by general procedure for the synthesis of 3ga. White solid, $81 \mathrm{mg}$, yield: $71 \%$ (purified by silica gel chromatography using PE/EA 10:1). mp 216-218 ${ }^{\circ} \mathrm{C} ;{ }^{1} \mathbf{H}$ NMR (500 MHz, DMSO-d6) $\delta 11.37$ (s, 1H), 11.22 (s, 1H), 8.44 (d, J = 1.6 Hz, 1H), $7.88(\mathrm{~d}, \mathrm{~J}=8.5 \mathrm{~Hz}, 1 \mathrm{H}), 7.63(\mathrm{dd}, \mathrm{J}=8.5,1.8 \mathrm{~Hz}, 1 \mathrm{H}), 7.53(\mathrm{~d}, \mathrm{~J}=8.5 \mathrm{~Hz}, 1 \mathrm{H}), 6.68$ $(\mathrm{d}, \mathrm{J}=8.5 \mathrm{~Hz}, 1 \mathrm{H}), 4.37(\mathrm{q}, \mathrm{J}=7.1 \mathrm{~Hz}, 2 \mathrm{H}), 1.35(\mathrm{t}, \mathrm{J}=7.1 \mathrm{~Hz}, 3 \mathrm{H}) .{ }^{13} \mathbf{C}$ NMR (125 MHz, DMSO-d6) $\delta 165.7,158.6,141.4,138.5,132.8,130.2,130.0,124.1,114.2$, 110.1, 105.3, 103.7, 82.6, 60.0, 14.5; HRMS m/z [M - H] calculated for $\mathrm{C}_{15} \mathrm{H}_{11} \mathrm{INO}_{3}$ : 379.9789, found: 379.9782 .

Ethyl 4-hydroxy-7-methyl-9H-carbazole-1-carboxylate (3ha)<smiles>CCOC(=O)c1ccc(O)c2c1[nH]c1cc(C)ccc12</smiles>

Following by general procedure for the synthesis of 3ha. White solid, $69 \mathrm{mg}$, yield: $85 \%$ (purified by silica gel chromatography using PE/EA 10:1). mp 208-210 ${ }^{\circ} \mathrm{C} ;{ }^{\mathbf{1}} \mathbf{H}$ NMR (500 MHz, DMSO-d6) $\delta 11.07$ (s, 1H), 10.92 (s, 1H), 8.01 (d, J = 7.9 Hz, 1H), $7.80(\mathrm{~d}, \mathrm{~J}=8.4 \mathrm{~Hz}, 1 \mathrm{H}), 7.47(\mathrm{~s}, 1 \mathrm{H}), 6.99(\mathrm{~d}, \mathrm{~J}=7.9 \mathrm{~Hz}, 1 \mathrm{H}), 6.63(\mathrm{~d}, \mathrm{~J}=8.4 \mathrm{~Hz}$, 1H), 4.37 (q, J = 7.1 Hz, 2H), 2.44 (s, 3H), 1.36 (t, J = 7.1 Hz, 3H). ${ }^{13}$ C NMR (125 MHz, DMSO-d6) $\delta 165.9,158.1,141.5,139.7,134.2,128.9,121.6,121.0,119.2$, 111.6, 111.3, 105.0, 103.4, 59.8, 21.8, 14.5; HRMS m/z $[\mathrm{M}-\mathrm{H}]^{-}$calculated for $\mathrm{C}_{16} \mathrm{H}_{14} \mathrm{NO}_{3}: 268.0979$, found: 268.0972 .

Ethyl 7-methyl 4-hydroxy-9H-carbazole-1,7-dicarboxylate (3ia)<smiles>CCOC(=O)c1ccc(O)c2c1[nH]c1cc(C(=O)OC)ccc12</smiles> 
Following by general procedure for the synthesis of 3ia. White solid, $62 \mathrm{mg}$, yield: $66 \%$ (purified by silica gel chromatography using PE/EA 10:1). mp 210-212 ${ }^{\circ} \mathrm{C} ;{ }^{1} \mathbf{H}$ NMR (500 MHz, DMSO-d6) $\delta 11.49$ (s, 1H), 11.31 (s, 1H), 8.36 (d, J = 0.9 Hz, 1H), 8.23 $(\mathrm{d}, \mathrm{J}=8.2 \mathrm{~Hz}, 1 \mathrm{H}), 7.93(\mathrm{~d}, \mathrm{~J}=8.4 \mathrm{~Hz}, 1 \mathrm{H}), 7.81(\mathrm{dd}, \mathrm{J}=8.2,1.5 \mathrm{~Hz}, 1 \mathrm{H}), 6.70(\mathrm{~d}, \mathrm{~J}$ $=8.5 \mathrm{~Hz}, 1 \mathrm{H}), 4.39(\mathrm{q}, \mathrm{J}=7.1 \mathrm{~Hz}, 2 \mathrm{H}), 3.88(\mathrm{~s}, 3 \mathrm{H}), 1.37(\mathrm{t}, \mathrm{J}=7.1 \mathrm{~Hz}, 3 \mathrm{H}) .{ }^{13} \mathrm{C}$ NMR (125 MHz, DMSO-d6) $\delta$ 167.0, 165.6, 159.2, 142.6, 138.7, 131.1, 125.7, 125.2, $121.7,120.4,113.2,110.8,105.2,103.8,60.0,52.0,14.5 ; \mathbf{H R M S ~ m / z ~}[\mathrm{M}-\mathrm{H}]^{-}$ calculated for $\mathrm{C}_{17} \mathrm{H}_{14} \mathrm{NO}_{5}: 312.0877$, found: 312.0875 .

\section{Ethyl 7-fluoro-4-hydroxy-9H-carbazole-1-carboxylate (3ja)}<smiles>CCOC(=O)c1ccc(O)c2c1[nH]c1cc(F)ccc12</smiles>

Following by general procedure for the synthesis of $\mathbf{3 j a}$. White solid, $71 \mathrm{mg}$, yield: $87 \%$ (purified by silica gel chromatography using PE/EA 10:1). mp 160-162 ${ }^{\circ} \mathrm{C} ;{ }^{1} \mathbf{H}$ NMR (500 MHz, DMSO-d6) $\delta 11.34$ (s, 1H), 11.07 (s, 1H), 8.10 (dd, J = 8.5, 5.7 Hz, 1H), $7.83(\mathrm{~d}, \mathrm{~J}=8.4 \mathrm{~Hz}, 1 \mathrm{H}), 7.42(\mathrm{dd}, \mathrm{J}=10.1,2.4 \mathrm{~Hz}, 1 \mathrm{H}), 7.00(\mathrm{ddd}, \mathrm{J}=9.8,8.6,2.4$ $\mathrm{Hz}, 1 \mathrm{H}), 6.67(\mathrm{~d}, \mathrm{~J}=8.5 \mathrm{~Hz}, 1 \mathrm{H}), 4.38(\mathrm{q}, \mathrm{J}=7.1 \mathrm{~Hz}, 2 \mathrm{H}), 1.35$ (t, J = 7.1 Hz, 3H). ${ }^{13}$ C NMR (125 MHz, DMSO-d6) $\delta$ 165.8, 160.5 (d, J = 237.9 Hz), 158.1, 142.0, $140.0(\mathrm{~d}, \mathrm{~J}=13.1 \mathrm{~Hz}), 129.2,123.0(\mathrm{~d}, \mathrm{~J}=10.2 \mathrm{~Hz}), 118.2,110.8,107.3(\mathrm{~d}, \mathrm{~J}=23.8$ Hz), 105.4, 103.6, 98.2 (d, J = 26.5 Hz), 59.9, 14.5. HRMS m/z [M - H] ${ }^{-}$calculated for $\mathrm{C}_{15} \mathrm{H}_{11} \mathrm{FNO}_{3}$ : 272.0728, found: 272.0721 .

\section{Ethyl 7-chloro-4-hydroxy-9H-carbazole-1-carboxylate (3ka)}

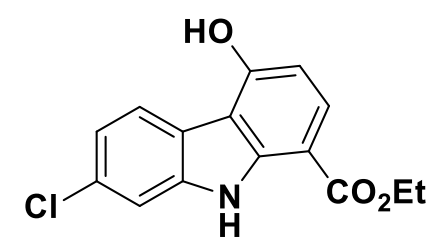

Following by general procedure for the synthesis of 3ka. White solid, $66 \mathrm{mg}$, yield: $76 \%$ (purified by silica gel chromatography using PE/EA 10:1). mp 198-200 ${ }^{\circ} \mathrm{C} ;{ }^{1} \mathbf{H}$ NMR (500 MHz, DMSO-d6) $\delta 11.37$ (s, 1H), 11.17 (s, 1H), 8.11 (d, J = 8.3 Hz, 1H), 
$7.87(\mathrm{~d}, \mathrm{~J}=8.5 \mathrm{~Hz}, 1 \mathrm{H}), 7.71(\mathrm{~d}, \mathrm{~J}=1.8 \mathrm{~Hz}, 1 \mathrm{H}), 7.19(\mathrm{dd}, \mathrm{J}=8.3,2.0 \mathrm{~Hz}, 1 \mathrm{H}), 6.69$ $(\mathrm{d}, \mathrm{J}=8.5 \mathrm{~Hz}, 1 \mathrm{H}), 4.38(\mathrm{q}, \mathrm{J}=7.1 \mathrm{~Hz}, 2 \mathrm{H}), 1.35(\mathrm{t}, \mathrm{J}=7.1 \mathrm{~Hz}, 3 \mathrm{H}) .{ }^{13} \mathrm{C}$ NMR $(125$ MHz, DMSO-d6) $\delta 165.7,158.4,141.8,140.0,129.9,129.2,123.1,120.3,119.6$, 111.4, 110.8, 105.4, 103.7, 60.0, 14.5; HRMS m/z $[\mathrm{M}-\mathrm{H}]^{-}$calculated for $\mathrm{C}_{15} \mathrm{H}_{11} \mathrm{ClNO}_{3}: 288.0433$, found: 288.0430 .

Ethyl 4-hydroxy-8-methoxy-9H-carbazole-1-carboxylate (3la)<smiles>CCOC(=O)c1ccc(O)c2c1[nH]c1c(OC)cccc12</smiles>

Following by general procedure for the synthesis of 3la. White solid, $65 \mathrm{mg}$, yield: 76\% (purified by silica gel chromatography using PE/EA 10:1). mp 208-210 ${ }^{\circ} \mathrm{C} ;{ }^{1} \mathbf{H}$ NMR (500 MHz, DMSO-d6) $\delta 11.13$ (s, 1H), 9.97 (s, 1H), 7.87 (d, J = 8.4 Hz, 1H), 7.79 (d, $\mathrm{J}=7.8 \mathrm{~Hz}, 1 \mathrm{H}), 7.17(\mathrm{t}, \mathrm{J}=7.9 \mathrm{~Hz}, 1 \mathrm{H}), 7.02(\mathrm{~d}, \mathrm{~J}=7.6 \mathrm{~Hz}, 1 \mathrm{H}), 6.71(\mathrm{~d}, \mathrm{~J}=8.4 \mathrm{~Hz}$, 1H), 4.39 (q, J = $7.1 \mathrm{~Hz}, 2 \mathrm{H}), 1.37$ (t, J = 7.1 Hz, 3H). ${ }^{13} \mathbf{C}$ NMR (125 MHz, DMSO-d6) $\delta 166.8,159.1,145.6,141.6,130.0,128.5,123.1,121.2,115.2,112.2$, 106.7, 106.0, 103.9, 60.6, 56.1, 14.8; HRMS m/z [M - H] $]^{-}$calculated for $\mathrm{C}_{16} \mathrm{H}_{14} \mathrm{NO}_{4}$ : 284.0928, found: 284.0923 .

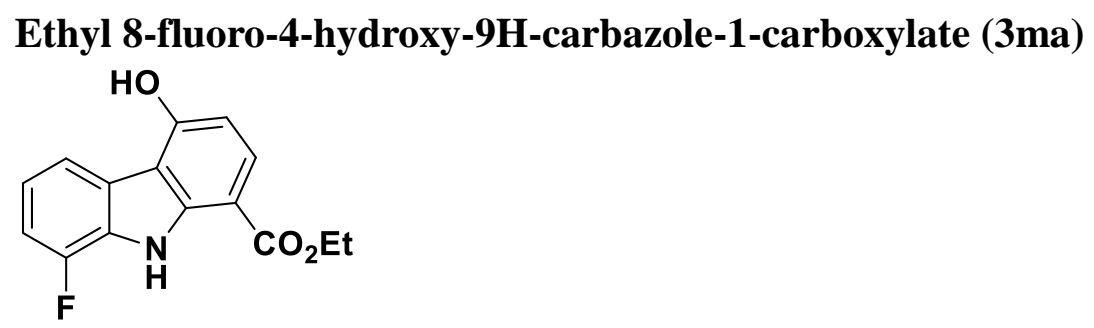

Following by general procedure for the synthesis of 3ma. White solid, $66 \mathrm{mg}$, yield: $81 \%$ (purified by silica gel chromatography using PE/EA 10:1). mp 166-168 ${ }^{\circ} \mathrm{C} ;{ }^{1} \mathbf{H}$ NMR (600 MHz, DMSO-d6) $\delta 11.23$ (s, 1H), 10.80 (s, 1H), 7.98 (d, J = 7.7 Hz, 1H), $7.89(\mathrm{~d}, \mathrm{~J}=8.4 \mathrm{~Hz}, 1 \mathrm{H}), 7.22(\mathrm{dd}, \mathrm{J}=11.2,7.9 \mathrm{~Hz}, 1 \mathrm{H}), 7.16(\mathrm{td}, \mathrm{J}=7.8,4.6 \mathrm{~Hz}, 1 \mathrm{H})$, $6.71(\mathrm{~d}, \mathrm{~J}=8.4 \mathrm{~Hz}, 1 \mathrm{H}), 4.38(\mathrm{q}, \mathrm{J}=7.1 \mathrm{~Hz}, 2 \mathrm{H}), 1.34(\mathrm{t}, \mathrm{J}=7.1 \mathrm{~Hz}, 3 \mathrm{H}) .{ }^{13} \mathbf{C} \mathbf{N M R}$ (150 MHz, DMSO-d6) $\delta$ 166.2, 159.1, 148.7 (d, J = 243.7 Hz), 142.0, 130.9, 126.8 (d, $\mathrm{J}=12.8 \mathrm{~Hz}), 125.9(\mathrm{~d}, \mathrm{~J}=5.1 \mathrm{~Hz}), 120.8(\mathrm{~d}, \mathrm{~J}=5.8 \mathrm{~Hz}), 118.6,112.0,111.0(\mathrm{~d}, \mathrm{~J}=$ 
16.3 Hz), 106.2, 104.6, 60.6, 14.9. HRMS m/z $[\mathrm{M}-\mathrm{H}]^{-}$calculated for $\mathrm{C}_{15} \mathrm{H}_{11} \mathrm{FNO}_{3}$ : 272.0728, found: 272.0724 .

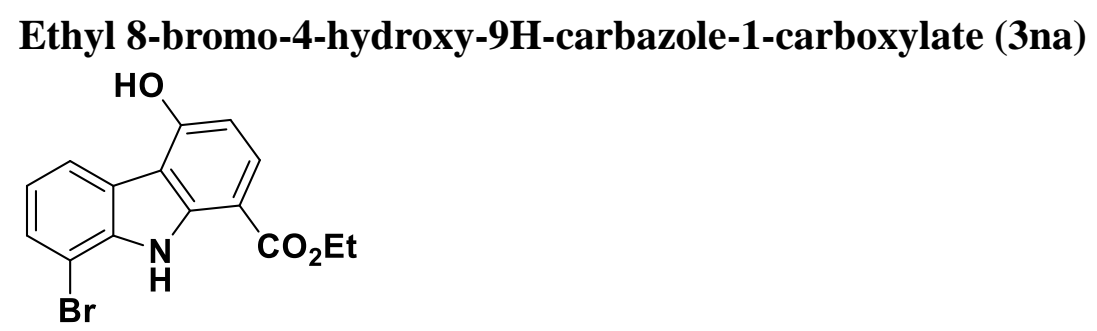

Following by general procedure for the synthesis of 3na. White solid, $65 \mathrm{mg}$, yield: $65 \%$ (purified by silica gel chromatography using PE/EA 10:1). mp 208-210 ${ }^{\circ} \mathrm{C} ;{ }^{1} \mathbf{H}$ NMR (500 MHz, DMSO-d6) $\delta 11.39$ (s, 1H), 9.98 (s, 1H), 8.18 (d, J = 7.7 Hz, 1H), $7.92(\mathrm{~d}, \mathrm{~J}=8.5 \mathrm{~Hz}, 1 \mathrm{H}), 7.64(\mathrm{~d}, \mathrm{~J}=7.8 \mathrm{~Hz}, 1 \mathrm{H}), 7.21(\mathrm{t}, \mathrm{J}=7.8 \mathrm{~Hz}, 1 \mathrm{H}), 6.77(\mathrm{~d}, \mathrm{~J}=$ $8.5 \mathrm{~Hz}, 1 \mathrm{H}), 4.40$ (q, J = 7.1 Hz, 2H), 1.40 (t, J = 7.1 Hz, 3H). ${ }^{13} \mathbf{C}$ NMR (125 MHz, DMSO-d6) $\delta 166.6,159.4,141.8,137.0,130.7,127.9,123.6,122.2,122.0,112.0$, 106.8, 104.1, 103.7, 60.9, 14.8; HRMS m/z $[\mathrm{M}-\mathrm{H}]^{-}$calculated for $\mathrm{C}_{15} \mathrm{H}_{11} \mathrm{BrNO}_{3}$ : 331.9928, found: 331.9922 .

\section{Ethyl 4-hydroxy-5-methyl-9H-carbazole-1-carboxylate (3oa)}<smiles>CCOC(=O)c1ccc(O)c2c1[nH]c1cccc(C)c12</smiles>

Following by general procedure for the synthesis of 3oa. White solid, $51 \mathrm{mg}$, yield: $63 \%$ (purified by silica gel chromatography using PE/EA 10:1). mp 206-208 ${ }^{\circ} \mathrm{C} ;{ }^{\mathbf{1}} \mathbf{H}$ NMR (600 MHz, DMSO-d6) $\delta 11.25$ (s, 1H), 10.82 (s, 1H), 7.82 (d, J = 8.4 Hz, 1H), $7.53(\mathrm{~d}, \mathrm{~J}=8.0 \mathrm{~Hz}, 1 \mathrm{H}), 7.19(\mathrm{t}, \mathrm{J}=7.6 \mathrm{~Hz}, 1 \mathrm{H}), 6.90(\mathrm{~d}, \mathrm{~J}=7.2 \mathrm{~Hz}, 1 \mathrm{H}), 6.61(\mathrm{~d}, \mathrm{~J}=$ $8.4 \mathrm{~Hz}, 1 \mathrm{H}), 4.35(\mathrm{q}, \mathrm{J}=7.1 \mathrm{~Hz}, 2 \mathrm{H}), 2.92(\mathrm{~s}, 3 \mathrm{H}), 1.34(\mathrm{t}, \mathrm{J}=7.1 \mathrm{~Hz}, 3 \mathrm{H}) .{ }^{13} \mathbf{C} \mathbf{N M R}$ (150 MHz, DMSO-d6) $\delta$ 166.5, 157.9, 142.5, 140.4, 132.6, 129.8, 125.6, 122.6, 120.9, 111.9, 110.0, 105.9, 103.3, 60.3, 24.2, 14.9; HRMS m/z [M - H] ${ }^{-}$calculated for $\mathrm{C}_{16} \mathrm{H}_{14} \mathrm{NO}_{3}$ : 268.0979, found: 268.0974. 
Ethyl 5-fluoro-4-hydroxy-9H-carbazole-1-carboxylate (3pa)<smiles>CCOC(=O)c1ccc(O)c2c1[nH]c1cccc(F)c12</smiles>

Following by general procedure for the synthesis of 3pa. White solid, $78 \mathrm{mg}$, yield: 95\% (purified by silica gel chromatography using PE/EA 10:1). mp 144-146 ${ }^{\circ} \mathrm{C} ;{ }^{1} \mathbf{H}$ NMR (500 MHz, DMSO-d6) $\delta 11.51$ (s, 1H), 10.81 (s, 1H), 7.89 (d, J = 8.5 Hz, 1H), $7.53(\mathrm{~d}, \mathrm{~J}=8.0 \mathrm{~Hz}, 1 \mathrm{H}), 7.33(\mathrm{td}, \mathrm{J}=8.0,4.9 \mathrm{~Hz}, 1 \mathrm{H}), 7.00-6.79(\mathrm{~m}, 1 \mathrm{H}), 6.69(\mathrm{~d}, \mathrm{~J}$ $=8.5 \mathrm{~Hz}, 1 \mathrm{H}), 4.38(\mathrm{q}, \mathrm{J}=7.1 \mathrm{~Hz}, 2 \mathrm{H}), 1.36(\mathrm{t}, \mathrm{J}=7.1 \mathrm{~Hz}, 3 \mathrm{H}) .{ }^{\mathbf{1 3}} \mathbf{C}$ NMR (125 MHz, DMSO-d6) $\delta 165.8,157.8,156.1(\mathrm{~d}, \mathrm{~J}=250.3 \mathrm{~Hz}), 142.0(\mathrm{~d}, \mathrm{~J}=10.2 \mathrm{~Hz}), 141.6$, 130.2, $126.2(\mathrm{~d}, \mathrm{~J}=8.0 \mathrm{~Hz}), 109.1,109.0(\mathrm{~d}, \mathrm{~J}=3.9 \mathrm{~Hz}), 108.1(\mathrm{~d}, \mathrm{~J}=3.1 \mathrm{~Hz}), 105.8$, 105.6, 103.2, 60.0, 14.5; HRMS m/z [M - H] calculated for $\mathrm{C}_{15} \mathrm{H}_{11} \mathrm{FNO}_{3}$ : 272.0728, found: 272.0723 .

\section{Ethyl 4-hydroxy-6,7-dimethoxy-9H-carbazole-1-carboxylate (3qa)}<smiles>CCOC(=O)c1ccc(O)c2c1[nH]c1cc(OC)c(OC)cc12</smiles>

Following by general procedure for the synthesis of 3qa. White solid, $90 \mathrm{mg}$, yield: 95\% (purified by silica gel chromatography using PE/EA 10:1). mp 220-222 ${ }^{\circ} \mathrm{C} ;{ }^{1} \mathbf{H}$ NMR (500 MHz, DMSO-d6) $\delta 10.94$ (s, 1H), 10.81 (s, 1H), 7.74 (d, J = 8.4 Hz, 1H), $7.62(\mathrm{~s}, 1 \mathrm{H}), 7.29$ (s, 1H), 6.60 (d, J = 8.4 Hz, 1H), 4.37 (q, J = 7.1 Hz, 2H), 3.82 (s, 3H), 3.81 (s, 3H), 1.35 (t, J = 7.1 Hz, 3H). ${ }^{13}$ C NMR (125 MHz, DMSO-d6) $\delta 166.0$, 157.4, 148.5, 144.0, 141.0, 134.0, 127.9, 113.4, 111.7, 104.9, 104.6, 103.5, 95.6, 59.8, 56.1, 55.6, 14.5; HRMS m/z [M - H] calculated for $\mathrm{C}_{17} \mathrm{H}_{16} \mathrm{NO}_{5}: 314.1034$, found: 314.1031. 
Ethyl 6-bromo-4-hydroxy-9-methyl-9H-carbazole-1-carboxylate (3ra)<smiles>CCOc1ccc(O)c2c3cc(Br)ccc3n(C)c12</smiles>

Following by general procedure for the synthesis of 3ra. White solid, $64 \mathrm{mg}$, yield: $62 \%$ (purified by silica gel chromatography using PE/EA 10:1). mp 208-210 ${ }^{\circ} \mathrm{C}$; ${ }^{1} \mathbf{H}$ NMR (500 MHz, DMSO-d6) $\delta 11.17$ (s, 1H), $8.31(\mathrm{~s}, 1 \mathrm{H}), 7.75$ (d, J= 8.4 Hz, 1H), 7.57 (d, $J=1.8 \mathrm{~Hz}, 2 \mathrm{H}), 6.70(\mathrm{~d}, J=8.4 \mathrm{~Hz}, 1 \mathrm{H}), 4.33(\mathrm{q}, J=7.1 \mathrm{~Hz}, 2 \mathrm{H}), 3.82(\mathrm{~s}, 3 \mathrm{H}), 1.34$ (t, $J=7.1 \mathrm{~Hz}, 3 \mathrm{H}) .{ }^{13} \mathrm{C}$ NMR (125 MHz, DMSO-d6) $\delta$ 166.2, 157.5, 141.3, 140.1, 131.5, 127.5, 124.1, 123.2, 111.9, 111.6, 111.0, 107.0, 105.1, 60.5, 33.8, 14.2; HRMS $\mathbf{m} / \mathbf{z}[\mathrm{M}-\mathrm{H}]^{-}$calculated for $\mathrm{C}_{16} \mathrm{H}_{13} \mathrm{BrNO}_{3}: 346.0084$, found: 346.0080 .

Methyl 4-hydroxy-9H-carbazole-1-carboxylate (3ab)<smiles>COC(=O)c1ccc(O)c2c1[nH]c1ccccc12</smiles>

Following by general procedure for the synthesis of 3ab. White solid, $61 \mathrm{mg}$, yield: $84 \%$ (purified by silica gel chromatography using PE/EA 10:1). mp 188-190 ${ }^{\circ} \mathrm{C} ;{ }^{1} \mathbf{H}$ NMR (500 MHz, DMSO-d6) $\delta 11.23$ (s, 1H), 11.03 (s, 1H), 8.18 (d, J = 7.7 Hz, 1H), $7.87(\mathrm{~d}, \mathrm{~J}=8.4 \mathrm{~Hz}, 1 \mathrm{H}), 7.69(\mathrm{~d}, \mathrm{~J}=8.1 \mathrm{~Hz}, 1 \mathrm{H}), 7.37(\mathrm{t}, \mathrm{J}=7.1 \mathrm{~Hz}, 1 \mathrm{H}), 7.19$ (t, J = $7.1 \mathrm{~Hz}, 1 \mathrm{H}), 6.69$ (d, J = 8.4 Hz, 1H), 3.93 (s, 3H). ${ }^{13}$ C NMR (125 MHz, DMSO-d6) $\delta 166.8,159.0,141.7,139.7,130.1,125.3,122.4,121.9,119.9,112.0,111.7,105.5$, 103.8, 51.9; HRMS m/z [M - H] calculated for $\mathrm{C}_{14} \mathrm{H}_{10} \mathrm{NO}_{3}$ : 240.0666, found: 240.0664 .

Methyl 6-fluoro-4-hydroxy-9H-carbazole-1-carboxylate (3db)<smiles>COC(=O)c1ccc(O)c2c1[nH]c1ccc(F)cc12</smiles> 
Following by general procedure for the synthesis of $\mathbf{3 d b}$. White solid, $51 \mathrm{mg}$, yield: $66 \%$ (purified by silica gel chromatography using PE/EA 10:1). mp 200-202 ${ }^{\circ} \mathrm{C} ;{ }^{1} \mathbf{H}$ NMR (600 MHz, DMSO-d6) $\delta 11.30$ (s, 1H), 11.21 (s, 1H), 7.88 (d, J = 8.4 Hz, 1H), $7.82(\mathrm{dd}, \mathrm{J}=9.2,2.5 \mathrm{~Hz}, 1 \mathrm{H}), 7.66(\mathrm{dd}, \mathrm{J}=8.8,4.6 \mathrm{~Hz}, 1 \mathrm{H}), 7.22(\mathrm{td}, \mathrm{J}=9.3,2.6 \mathrm{~Hz}$, 1H), 6.66 (d, J = 8.4 Hz, 1H), 3.90 (s, 3H). ${ }^{13}$ C NMR (150 MHz, DMSO-d6) $\delta 166.2$, 158.7, 156.7 (d, J = 232.6 Hz), 142.1, 135.7, 130.4, 121.8 (d, J = 10.5 Hz), 112.6 (d, J $=11.6 \mathrm{~Hz}), 112.4(\mathrm{~d}, \mathrm{~J}=4.1 \mathrm{~Hz}), 111.1(\mathrm{~d}, \mathrm{~J}=3.8 \mathrm{~Hz}), 107.1(\mathrm{~d}, \mathrm{~J}=24.4 \mathrm{~Hz}), 104.8$, 103.4, 51.4; HRMS m/z [M - H] calculated for $\mathrm{C}_{14} \mathrm{H}_{9} \mathrm{FNO}_{3}$ : 258.0572, found: 258.0567 .

\section{Isopropyl 4-hydroxy-9H-carbazole-1-carboxylate (3ac)}

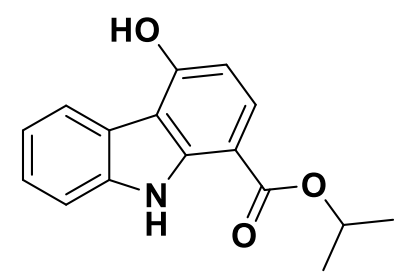

Following by general procedure for the synthesis of 3ac. White solid, $64 \mathrm{mg}$, yield: 79\% (purified by silica gel chromatography using PE/EA 10:1). mp 197-199 ${ }^{\circ} \mathrm{C} ;{ }^{1} \mathbf{H}$ NMR (500 MHz, DMSO-d6) $\delta 11.20$ (s, 1H), 11.00 (s, 1H), 8.17 (d, J = 7.7 Hz, 1H), 7.85 $(\mathrm{d}, \mathrm{J}=8.4 \mathrm{~Hz}, 1 \mathrm{H}), 7.71(\mathrm{~d}, \mathrm{~J}=8.1 \mathrm{~Hz}, 1 \mathrm{H}), 7.36(\mathrm{t}, \mathrm{J}=7.5 \mathrm{~Hz}, 1 \mathrm{H}), 7.19$ (t, J = 7.5 Hz, 1H), $6.67(\mathrm{~d}, \mathrm{~J}=8.4 \mathrm{~Hz}, 1 \mathrm{H}), 5.24$ (hept, 1H), $1.39(\mathrm{~d}, \mathrm{~J}=6.2 \mathrm{~Hz}, 6 \mathrm{H}) .{ }^{13} \mathbf{C} \mathbf{N M R}$ (125 MHz, DMSO-d6) $\delta$ 166.0, 158.8, 142.0, 139.7, 129.7, 125.2, 122.4, 121.8, 119.9, 112.2, 111.7, 105.3, 104.2, 67.6, 22.3×2; HRMS m/z [M - H] $]^{-}$calculated for $\mathrm{C}_{16} \mathrm{H}_{14} \mathrm{NO}_{3}$ : 268.0979, found: 268.0975.

\section{Isopropyl 6-fluoro-4-hydroxy-9H-carbazole-1-carboxylate (3dc)<smiles>CC(C)OC(=O)c1ccc(O)c2c1[nH]c1ccc(F)cc12</smiles>

Following by general procedure for the synthesis of 3dc. White solid, $66 \mathrm{mg}$, yield: $76 \%$ (purified by silica gel chromatography using PE/EA 10:1). mp 210-212 ${ }^{\circ} \mathrm{C} ;{ }^{1} \mathbf{H}$ NMR (500 MHz, DMSO-d6) $\delta 11.27$ (s, 1H), 11.15 (s, 1H), 7.85 (d, J = 8.4 Hz, 1H), 
$7.80(\mathrm{dd}, \mathrm{J}=9.3,2.6 \mathrm{~Hz}, 1 \mathrm{H}), 7.67(\mathrm{dd}, \mathrm{J}=8.8,4.6 \mathrm{~Hz}, 1 \mathrm{H}), 7.20(\mathrm{td}, \mathrm{J}=9.4,2.7 \mathrm{~Hz}$,

1H), $6.64(\mathrm{~d}, \mathrm{~J}=8.5 \mathrm{~Hz}, 1 \mathrm{H}), 5.20$ (hept, $\mathrm{J}=6.2 \mathrm{~Hz}, 1 \mathrm{H}), 1.36(\mathrm{~d}, \mathrm{~J}=6.3 \mathrm{~Hz}, 6 \mathrm{H})$.

${ }^{13}$ C NMR (125 MHz, DMSO-d6) $\delta$ 165.3, 158.6, 156.8 (d, J = 232.6 Hz), 142.4, 135.8, 130.0, 121.7 (d, J = 10.5 Hz), $112.6(\mathrm{~d}, \mathrm{~J}=9.2 \mathrm{~Hz}), 112.4(\mathrm{~d}, \mathrm{~J}=25.1 \mathrm{~Hz})$, $111.1(\mathrm{~d}, \mathrm{~J}=3.7 \mathrm{~Hz}), 107.0(\mathrm{~d}, \mathrm{~J}=24.3 \mathrm{~Hz}), 104.6,103.9,67.2,21.9 \times 2 ; \mathbf{H R M S ~ m} / \mathbf{z}$ $[\mathrm{M}-\mathrm{H}]^{-}$calculated for $\mathrm{C}_{16} \mathrm{H}_{13} \mathrm{FNO}_{3}: 286.0885$, found: 286.0878 .

\section{Isopropyl 4-hydroxy-7-methyl-9H-carbazole-1-carboxylate (3hc)}

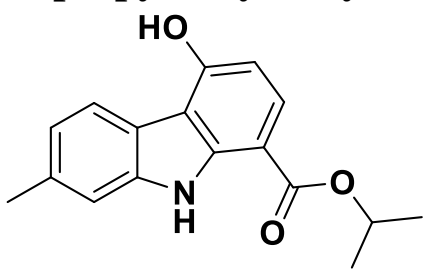

Following by general procedure for the synthesis of 3hc. White solid, $81 \mathrm{mg}$, yield: 95\% (purified by silica gel chromatography using PE/EA 10:1). mp 212-214 ${ }^{\circ} \mathrm{C} ;{ }^{\mathbf{1}} \mathbf{H}$ NMR (600 MHz, DMSO-d6) $\delta 11.08$ (s, 1H), 10.92 (s, 1H), 8.02 (d, J = 7.9 Hz, 1H), $7.80(\mathrm{~d}, \mathrm{~J}=8.4 \mathrm{~Hz}, 1 \mathrm{H}), 7.49(\mathrm{~s}, 1 \mathrm{H}), 6.99(\mathrm{~d}, \mathrm{~J}=7.8 \mathrm{~Hz}, 1 \mathrm{H}), 6.63(\mathrm{~d}, \mathrm{~J}=8.4 \mathrm{~Hz}$, 1H), 5.21 (hept, $\mathbf{J}=6.2 \mathrm{~Hz}, 1 \mathrm{H}), 2.44(\mathrm{~s}, 3 \mathrm{H}), 1.36(\mathrm{~d}, \mathrm{~J}=6.3 \mathrm{~Hz}, 6 \mathrm{H}) .{ }^{\mathbf{1 3}} \mathbf{C} \mathbf{~ N M R}$ (150 MHz, DMSO-d6) $\delta$ 165.5, 158.0, 141.6, 139.7, 134.2, 128.8, 121.6, 120.9, 119.2, 111.7, 111.3, 104.8, 103.7, 67.1, 21.9×2, 21.8; HRMS m/z [M - H] ${ }^{-}$calculated for $\mathrm{C}_{17} \mathrm{H}_{16} \mathrm{NO}_{3}$ : 282.1136, found: 282.1133 .

\section{Tert-butyl 4-hydroxy-9H-carbazole-1-carboxylate (3ad) HO<smiles>CCc1ccc(C(=O)OC(C)(C)C)c2[nH]c3ccccc3c12</smiles>

Following by general procedure for the synthesis of 3ad. White solid, $72 \mathrm{mg}$, yield: $85 \%$ (purified by silica gel chromatography using PE/EA 10:1). mp 160-162 ${ }^{\circ} \mathrm{C} ;{ }^{1} \mathbf{H}$ NMR (500 MHz, DMSO-d6) $\delta 11.23$ (s, 1H), 10.95 (s, 1H), 8.18 (d, J = 7.7 Hz, 1H), $7.81(\mathrm{~d}, \mathrm{~J}=8.4 \mathrm{~Hz}, 1 \mathrm{H}), 7.71(\mathrm{~d}, \mathrm{~J}=8.1 \mathrm{~Hz}, 1 \mathrm{H}), 7.35(\mathrm{t}, \mathrm{J}=7.1 \mathrm{~Hz}, 1 \mathrm{H}), 7.18(\mathrm{t}, \mathrm{J}=$ $7.1 \mathrm{~Hz}, 1 \mathrm{H}), 6.66$ (d, J = $8.4 \mathrm{~Hz}, 1 \mathrm{H}), 1.63$ (s, 9H). ${ }^{13}$ C NMR (125 MHz, DMSO-d6) $\delta 166.0,158.6,142.1,139.7,129.8,125.2,122.4,121.8,119.8,112.2,111.7,105.2$, 
105.2, 80.3, 28.6×3; HRMS m/z [M - H] $]^{-}$calculated for $\mathrm{C}_{17} \mathrm{H}_{16} \mathrm{NO}_{3}: 282.1136$, found: 282.1132 .

Tert-butyl 6-fluoro-4-hydroxy-9H-carbazole-1-carboxylate (3dd)<smiles>CC(C)(C)OC(=O)c1ccc(O)c2c1[nH]c1ccc(F)cc12</smiles>

Following by general procedure for the synthesis of 3dd. White solid, $57 \mathrm{mg}$, yield: $63 \%$ (purified by silica gel chromatography using PE/EA 10:1). mp 182-184 ${ }^{\circ} \mathrm{C} ;{ }^{1} \mathbf{H}$ NMR (500 MHz, DMSO-d6) $\delta 11.31$ (s, 1H), 11.10 (s, 1H), 7.82 (dd, J = 8.9, 3.6 Hz, 2H), $7.68(\mathrm{dd}, \mathrm{J}=8.8,4.6 \mathrm{~Hz}, 1 \mathrm{H}), 7.21(\mathrm{td}, \mathrm{J}=9.3,2.7 \mathrm{~Hz}, 1 \mathrm{H}), 6.64(\mathrm{~d}, \mathrm{~J}=8.4 \mathrm{~Hz}$, 1H), 1.62 (s, 9H). ${ }^{13} \mathbf{C}$ NMR (125 MHz, DMSO-d6) $\delta 165.9,158.8,157.2(\mathrm{~d}, \mathrm{~J}=$ 232.4 Hz), 142.9, 136.2, 130.5, 122.1 (d, J = 10.5 Hz), 113.0 (d, J = 9.2 Hz), 112.8 (d, $\mathrm{J}=25.0 \mathrm{~Hz}), 111.5(\mathrm{~d}, \mathrm{~J}=3.8 \mathrm{~Hz}), 107.4(\mathrm{~d}, \mathrm{~J}=24.3 \mathrm{~Hz}), 105.4,104.9,80.4,28.6 \times 3$;

HRMS m/z [M - H] $]^{-}$calculated for $\mathrm{C}_{17} \mathrm{H}_{15} \mathrm{FNO}_{3}: 300.1041$, found: 300.1034 .

\section{Tert-butyl 4-hydroxy-7-methyl-9H-carbazole-1-carboxylate (3hd)}

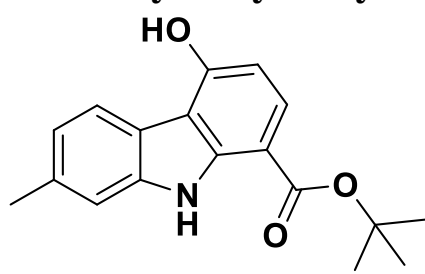

Following by general procedure for the synthesis of 3hd. White solid, $49 \mathrm{mg}$, yield: $55 \%$ (purified by silica gel chromatography using PE/EA 10:1). mp 188-190 ${ }^{\circ} \mathrm{C} ;{ }^{1} \mathbf{H}$ NMR (600 MHz, DMSO-d6) $\delta 11.09$ (s, 1H), 10.85 (s, 1H), 7.99 (d, J = 7.9 Hz, 1H), $7.73(\mathrm{~d}, \mathrm{~J}=8.4 \mathrm{~Hz}, 1 \mathrm{H}), 7.46(\mathrm{~s}, 1 \mathrm{H}), 6.98(\mathrm{~d}, \mathrm{~J}=7.9 \mathrm{~Hz}, 1 \mathrm{H}), 6.60(\mathrm{~d}, \mathrm{~J}=8.4 \mathrm{~Hz}$, 1H), 2.44 (s, 3H), 1.60 (s, 9H). ${ }^{13}$ C NMR (150 MHz, DMSO-d6) $\delta$ 165.6, 157.8, $141.6,139.7,134.1,128.9,121.6,120.9,119.1,111.6,111.2,104.7,104.6,79.8$, 28.1×3, 21.8; HRMS m/z [M - H] calculated for $\mathrm{C}_{18} \mathrm{H}_{18} \mathrm{NO}_{3}$ : 296.1292, found: 296.1290. 


\section{Benzyl 4-hydroxy-9H-carbazole-1-carboxylate (3ae)}<smiles>O=C(OCc1ccccc1)c1ccc(O)c2c1[nH]c1ccccc12</smiles>

Following by general procedure for the synthesis of 3ae. White solid, $80 \mathrm{mg}$, yield: $84 \%$ (purified by silica gel chromatography using PE/EA 10:1). mp 198-200 ${ }^{\circ} \mathrm{C} ;{ }^{1} \mathbf{H}$ NMR (500 MHz, DMSO-d6) $\delta 11.33$ (s, 1H), 11.08 (s, 1H), 8.18 (d, J = 7.7 Hz, 1H), 7.91 $(\mathrm{d}, \mathrm{J}=8.5 \mathrm{~Hz}, 1 \mathrm{H}), 7.70(\mathrm{~d}, \mathrm{~J}=8.1 \mathrm{~Hz}, 1 \mathrm{H}), 7.52(\mathrm{~d}, \mathrm{~J}=7.2 \mathrm{~Hz}, 2 \mathrm{H}), 7.44-7.32(\mathrm{~m}$, 4H), 7.19 (t, J = 7.1 Hz, 1H), 6.69 (d, J = 8.5 Hz, 1H), $5.45(\mathrm{~s}, 2 \mathrm{H}) .{ }^{13} \mathbf{C}$ NMR (125 MHz, DMSO-d6) $\delta$ 166.1, 159.2, 142.0, 139.7, 137.4, 130.0, 129.0×2, 128.4, $128.3 \times 2,125.3,122.4,121.9,120.0,112.1,111.7,105.6,103.5,65.7 ; \mathbf{H R M S ~ m / z ~ [ M ~}$ - H] $]^{-}$calculated for $\mathrm{C}_{20} \mathrm{H}_{14} \mathrm{NO}_{3}: 316.0979$, found: 316.0978 .

\section{Benzyl 6-fluoro-4-hydroxy-9H-carbazole-1-carboxylate (3de)}

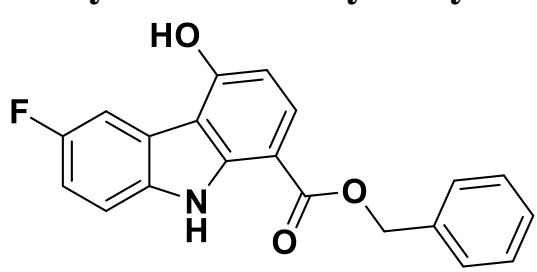

Following by general procedure for the synthesis of 3de. White solid, $73 \mathrm{mg}$, yield: $73 \%$ (purified by silica gel chromatography using PE/EA 10:1). mp 208-210 ${ }^{\circ} \mathrm{C} ;{ }^{1} \mathbf{H}$ NMR (500 MHz, DMSO-d6) $\delta 11.39$ (s, 1H), 11.22 (s, 1H), 7.92 (d, J = 8.5 Hz, 1H), $7.81(\mathrm{dd}, \mathrm{J}=9.3,2.7 \mathrm{~Hz}, 1 \mathrm{H}), 7.67(\mathrm{dd}, \mathrm{J}=8.8,4.6 \mathrm{~Hz}, 1 \mathrm{H}), 7.48(\mathrm{~d}, \mathrm{~J}=7.4 \mathrm{~Hz}, 2 \mathrm{H})$, $7.38(\mathrm{t}, \mathrm{J}=7.4 \mathrm{~Hz}, 2 \mathrm{H}), 7.32(\mathrm{t}, \mathrm{J}=7.3 \mathrm{~Hz}, 1 \mathrm{H}), 7.24-7.18(\mathrm{~m}, 1 \mathrm{H}), 6.66(\mathrm{~d}, \mathrm{~J}=8.5$ $\mathrm{Hz}, 1 \mathrm{H}), 5.42$ (s, 2H). ${ }^{13} \mathrm{C}$ NMR (125 MHz, DMSO-d6) $\delta 165.5,158.9,156.8$ (d, J = $232.5 \mathrm{~Hz}), 142.4,136.9,135.8,130.4,128.5 \times 2,127.9,127.8 \times 2,121.8(\mathrm{~d}, \mathrm{~J}=10.5$ $\mathrm{Hz}), 112.6(\mathrm{~d}, \mathrm{~J}=2.1 \mathrm{~Hz}), 112.5(\mathrm{~d}, \mathrm{~J}=18.7 \mathrm{~Hz}), 111.1(\mathrm{~d}, \mathrm{~J}=4.0 \mathrm{~Hz}), 107.1(\mathrm{~d}, \mathrm{~J}=$ 24.4 Hz), 104.9, 103.2, 65.3. HRMS m/z $[\mathrm{M}-\mathrm{H}]^{-}$calculated for $\mathrm{C}_{20} \mathrm{H}_{13} \mathrm{FNO}_{3}$ : 334.0885, found: 334.0882 . 
Benzyl 4-hydroxy-7-methyl-9H-carbazole-1-carboxylate (3he)<smiles>Cc1ccc2c(c1)[nH]c1c(C(=O)OCc3ccccc3)ccc(O)c12</smiles>

Following by general procedure for the synthesis of 3he. White solid, $65 \mathrm{mg}$, yield: $65 \%$ (purified by silica gel chromatography using PE/EA 10:1). mp 210-212 ${ }^{\circ} \mathrm{C} ;{ }^{1} \mathbf{H}$ NMR (500 MHz, DMSO-d6) $\delta 11.19$ (s, 1H), 10.98 (s, 1H), 8.04 (d, J = 7.9 Hz, 1H), $7.87(\mathrm{~d}, \mathrm{~J}=8.5 \mathrm{~Hz}, 1 \mathrm{H}), 7.53-7.49(\mathrm{~m}, 3 \mathrm{H}), 7.41(\mathrm{t}, \mathrm{J}=7.4 \mathrm{~Hz}, 2 \mathrm{H}), 7.34$ (t, J = 7.3 Hz, 1H), $7.02(\mathrm{~d}, \mathrm{~J}=7.9 \mathrm{~Hz}, 1 \mathrm{H}), 6.66(\mathrm{~d}, \mathrm{~J}=8.5 \mathrm{~Hz}, 1 \mathrm{H}), 5.44(\mathrm{~s}, 2 \mathrm{H}), 2.47(\mathrm{~s}, 3 \mathrm{H})$. ${ }^{13}$ C NMR (125 MHz, DMSO-d6) $\delta$ 166.1, 158.7, 142.1, 140.1, 137.4, 134.7, 129.5, $128.9 \times 2,128.34,128.26 \times 2,122.1,121.4,119.6,112.1,111.8,105.5,103.5,65.6,22.2$

HRMS m/z [M - H] $]^{-}$calculated for $\mathrm{C}_{21} \mathrm{H}_{16} \mathrm{NO}_{3}: 330.1136$, found: 330.1132 .

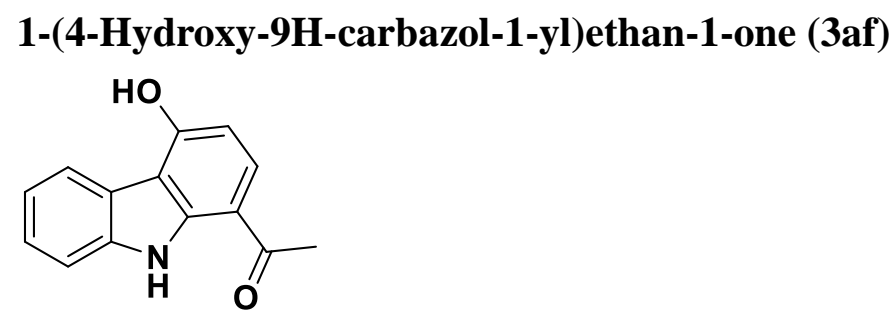

Following by general procedure for the synthesis of 3af. White solid, $48 \mathrm{mg}$, yield: $71 \%$ (purified by silica gel chromatography using PE/EA 3:1). mp 220-222 ${ }^{\circ} \mathrm{C} ;{ }^{1} \mathbf{H}$ NMR (500 MHz, DMSO-d6) $\delta 11.58$ (s, 1H), 11.12 (s, 1H), 8.17 (d, J = 7.7 Hz, 1H), 7.95 $(\mathrm{d}, \mathrm{J}=8.5 \mathrm{~Hz}, 1 \mathrm{H}), 7.73(\mathrm{~d}, \mathrm{~J}=8.1 \mathrm{~Hz}, 1 \mathrm{H}), 7.35(\mathrm{t}, \mathrm{J}=7.6 \mathrm{~Hz}, 1 \mathrm{H}), 7.19(\mathrm{t}, \mathrm{J}=7.5$ $\mathrm{Hz}, 1 \mathrm{H}), 6.68(\mathrm{~d}, \mathrm{~J}=8.4 \mathrm{~Hz}, 1 \mathrm{H}), 2.61(\mathrm{~s}, 3 \mathrm{H}) .{ }^{13} \mathrm{C}$ NMR (125 MHz, DMSO-d6) $\delta$ $196.8,158.8,140.6,139.3,131.1,124.7,121.8,121.0,119.6,112.6,111.9,111.0$, 104.6, 26.4; HRMS m/z $[\mathrm{M}-\mathrm{H}]^{-}$calculated for $\mathrm{C}_{14} \mathrm{H}_{10} \mathrm{NO}_{2}$ : 224.0717, found: 224.0712 .

1-(4-Hydroxy-7-methyl-9H-carbazol-1-yl)ethan-1-one (3hf)<smiles>CC(=O)c1ccc(O)c2c1[nH]c1cc(C)ccc12</smiles> 
Following by general procedure for the synthesis of 3df. White solid, $61 \mathrm{mg}$, yield: 85\% (purified by silica gel chromatography using PE/EA 3:1). mp 226-228 ${ }^{\circ} \mathrm{C}$; ${ }^{1} \mathbf{H}$ NMR (500 MHz, DMSO-d6) $\delta 11.44$ (s, 1H), 11.02 (s, 1H), 8.02 (d, J = 7.9 Hz, 1H), 7.90 $(\mathrm{d}, \mathrm{J}=8.5 \mathrm{~Hz}, 1 \mathrm{H}), 7.52(\mathrm{~s}, 1 \mathrm{H}), 7.02(\mathrm{~d}, \mathrm{~J}=7.9 \mathrm{~Hz}, 1 \mathrm{H}), 6.65(\mathrm{~d}, \mathrm{~J}=8.4 \mathrm{~Hz}, 1 \mathrm{H})$, 2.60 (s, 3H), 2.46 (s, 3H). ${ }^{13}$ C NMR (125 MHz, DMSO-d6) $\delta$ 197.2, 158.8, 141.1, $140.2,134.5,131.0,122.0,121.5,119.3,113.1,112.3,111.6,105.1,26.9,22.2$; HRMS m/z [M - H] ${ }^{-}$calculated for $\mathrm{C}_{15} \mathrm{H}_{12} \mathrm{NO}_{2}: 238.0874$, found: 238.0870 . 


\section{General methods for mechanism study and their spectral data}

\section{H/D exchange experiment}<smiles>CN(C)/C=C/C(=O)c1c[nH]c2ccccc12</smiles>

$1 \mathrm{a}$

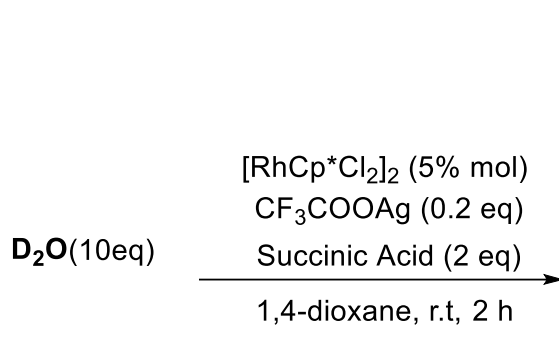

tube

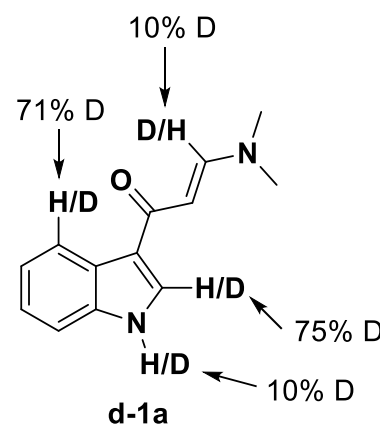

charged

with

(E)-3-(dimethylamino)-1-(1H-indol-3-yl)prop-2-en-1-one (1a, $64.3 \mathrm{mg}, 0.3 \mathrm{mmol})$ and $\mathrm{D}_{2} \mathrm{O}(60.1 \mathrm{mg}, 3 \mathrm{mmol}),\left[\mathrm{Cp}^{*} \mathrm{RhCl}_{2}\right]_{2}(9.3 \mathrm{mg}, 5 \mathrm{~mol} \%), \mathrm{CF}_{3} \mathrm{COOAg}(13.3 \mathrm{mg}$, $20 \mathrm{~mol} \%)$ Succinic acid $(70.9 \mathrm{mg}, 0.6 \mathrm{mmol})$ and 1,4-dioxane $(1 \mathrm{~mL})$. The reaction mixture was stirred at room temperature for $2 \mathrm{~h}$. After that, the solvent was removed under reduced pressure and the residue was purified by silica gel chromatography using DCM/MeOH (15:1) to afford the product d-1a as yellow solid. Yield: 70\%.

\section{${ }^{1}$ H NMR (500 MHz, DMSO-d6)}

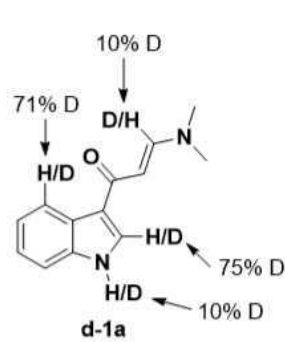

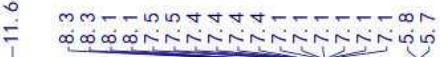

i

d-1a
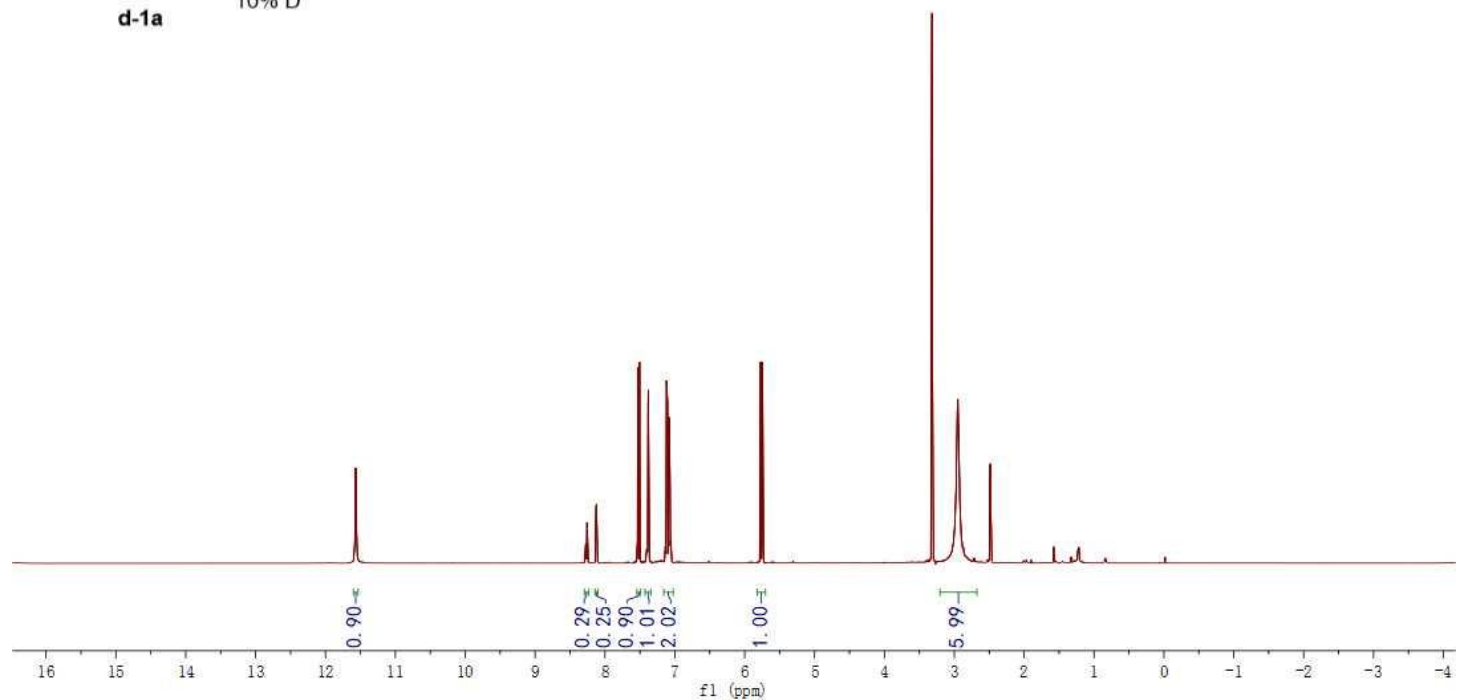


\section{Competition experiment}<smiles></smiles>

1b, $\mathrm{R}^{1}=\mathrm{CH}_{3}, 0.3 \mathrm{mmol}$ 1d, $R^{2}=F, 0.3 \mathrm{mmol}$

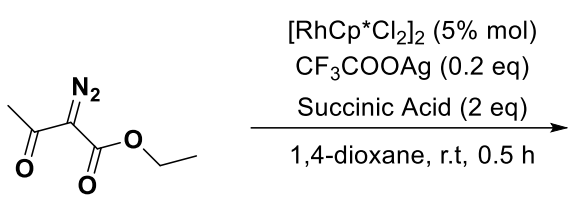

2a, $0.45 \mathrm{mmol}$<smiles>[R]C(=[W])c1ccc2[nH]c3c(C(=O)OCC)ccc(O)c3c2c1</smiles>

$3 \mathbf{b a} / 3 \mathbf{d a}=0.66$
A
pressure
tube
was
charged
with

(E)-3-(dimethylamino)-1-(5-methyl-1H-indol-3-yl)prop-2-en-1-one (1b, $68.5 \mathrm{mg}, 0.3$ mmol), (E)-3-(dimethylamino)-1-(5-fluoro-1H-indol-3-yl)prop-2-en-1-one (1d, 69.6 $\mathrm{mg}, \quad 0.3 \mathrm{mmol}$ ) and ethyl 2-diazo-3-oxobutanoate (2a, $70.3 \mathrm{mg}, 0.45 \mathrm{mmol}$ ), $\left[\mathrm{Cp}^{*} \mathrm{RhCl}_{2}\right]_{2}(9.3 \mathrm{mg}, 5 \mathrm{~mol} \%), \mathrm{CF}_{3} \mathrm{COOAg}(13.3 \mathrm{mg}, 20 \mathrm{~mol} \%)$ Succinic acid (70.9 $\mathrm{mg}, 0.6 \mathrm{mmol})$ and 1,4-dioxane $(1 \mathrm{~mL})$. The reaction mixture was stirred at room temperature for $0.5 \mathrm{~h}$. After that, the solvent was removed under reduced pressure and the residue was purified by silica gel chromatography using PE/EA (10:1) to afford the mixture of 3ba and 3da as white solid. The ratio of 3ba: 3da $=0.66: 1$ was determined on the basis of ${ }^{1} \mathrm{H}$ NMR analysis.

${ }^{1}$ H NMR (600 MHz, DMSO-d6)

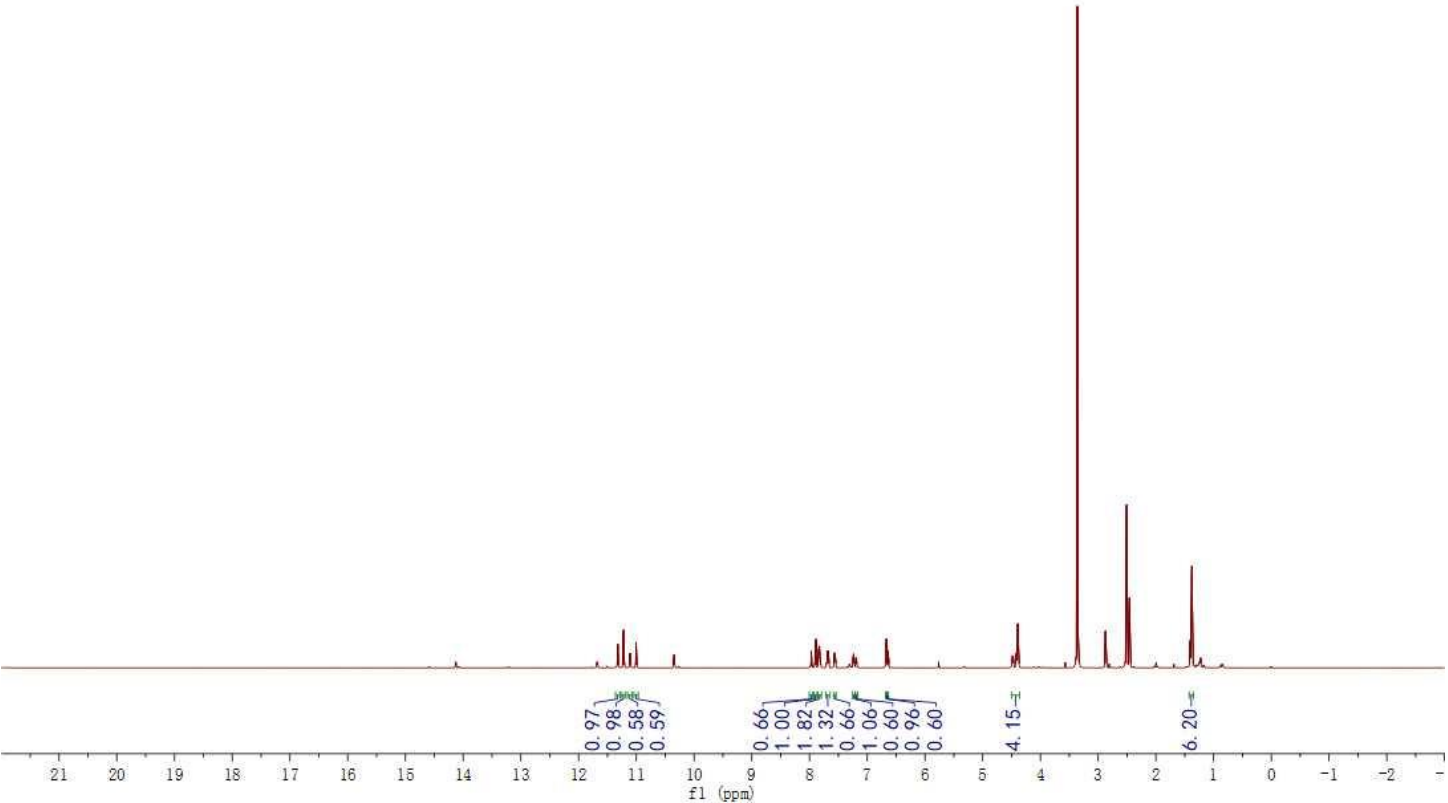




\section{General methods for applications and their spectral data}

\section{4-Hydroxy-9H-carbazole-1-carboxylic acid (4)}<smiles>CCOC(=O)c1ccc(O)c2c1[nH]c1ccccc12</smiles>

3aa

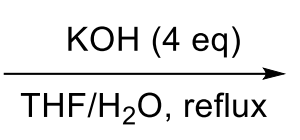

$\mathrm{HF} / \mathrm{H}_{2} \mathrm{O}$, reflux<smiles>O=C(O)c1ccc(O)c2c1[nH]c1ccccc12</smiles>

4

A tube was charged with 3aa $(1 \mathrm{mmol})$, potassium hydroxide $(4 \mathrm{mmol})$ and $\mathrm{THF} / \mathrm{H}_{2} \mathrm{O}(20 \mathrm{~mL})$. The reaction mixture was stirred under reflux in an oil bath until the starting material was fully consumed $(48 \mathrm{~h})$. After that, the solvent was removed under reduced pressure and the residue was purified by silica gel chromatography using PE/EA (3:1) to afford the product as white solid. Yield: $80 \%$. mp 230-232 ${ }^{\circ} \mathrm{C}$; ${ }^{1}$ H NMR (500 MHz, DMSO-d6) $\delta 12.60$ (s, 1H), 11.15 (s, 1H), 10.95 (s, 1H), 8.17 (d, $\mathrm{J}=7.7 \mathrm{~Hz}, 1 \mathrm{H}), 7.84(\mathrm{~d}, \mathrm{~J}=8.4 \mathrm{~Hz}, 1 \mathrm{H}), 7.71(\mathrm{~d}, \mathrm{~J}=8.1 \mathrm{~Hz}, 1 \mathrm{H}), 7.35(\mathrm{t}, \mathrm{J}=7.7 \mathrm{~Hz}$, 1H), $7.17(\mathrm{t}, \mathrm{J}=7.5 \mathrm{~Hz}, 1 \mathrm{H}), 6.67(\mathrm{~d}, \mathrm{~J}=8.4 \mathrm{~Hz}, 1 \mathrm{H}) .{ }^{13} \mathrm{C}$ NMR (125 MHz, DMSO-d6) $\delta 167.2,157.7,141.1,138.6,129.3,124.1,121.3,120.9,118.7,111.1$, 110.6, 104.2, 103.6; HRMS m/z [M - H] ${ }^{-}$calculated for $\mathrm{C}_{13} \mathrm{H}_{8} \mathrm{NO}_{3}: 226.0510$, found: 226.0508 .

6-Hydroxy-3,4-diphenylpyrano[3,4-a]carbazol-1(11H)-one (5)<smiles>O=C(O)c1ccc(O)c2c1[nH]c1ccccc12</smiles>

4

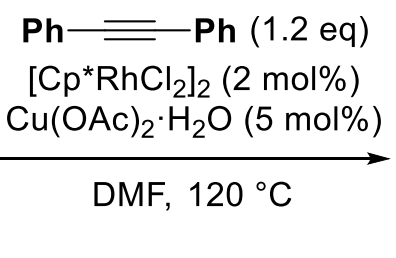

$\left[\mathrm{Cp}^{*} \mathrm{RhCl}_{2}\right]_{2}(2 \mathrm{~mol} \%)$

DMF, $120{ }^{\circ} \mathrm{C}$

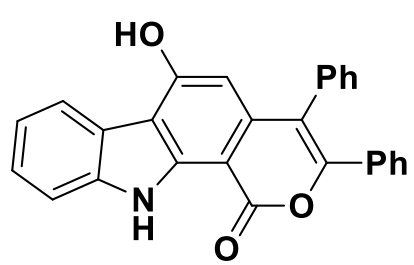

5

A pressure tube was charged with $4(68 \mathrm{mg}, 0.3 \mathrm{mmol})$, 1,2-diphenylethyne (64 mg, $0.36 \mathrm{mmol}),\left[\mathrm{Cp} * \mathrm{RhCl}_{2}\right]_{2}(3.7 \mathrm{mg}, 2 \mathrm{~mol} \%), \mathrm{Cu}(\mathrm{OAc})_{2} \cdot \mathrm{H}_{2} \mathrm{O}(3 \mathrm{mg}, 5 \% \mathrm{~mol})$ and DMF $(1 \mathrm{~mL})$. The reaction mixture was stirred at $120^{\circ} \mathrm{C}$ in an oil bath for $12 \mathrm{~h}$. After that, the solvent was removed under reduced pressure and the residue was purified by silica gel chromatography using PE/EA (10:1) to afford the product as white solid. Yield: 43\%. mp 200-202 ${ }^{\circ} \mathrm{C} ;{ }^{1}$ H NMR (500 MHz, DMSO-d6) $\delta 11.84$ (s, 1H), 11.28 
(s, 1H), $8.17(\mathrm{~d}, \mathrm{~J}=7.7 \mathrm{~Hz}, 1 \mathrm{H}), 7.83(\mathrm{~d}, \mathrm{~J}=8.1 \mathrm{~Hz}, 1 \mathrm{H}), 7.52-7.22(\mathrm{~m}, 12 \mathrm{H}), 6.36$ (s, 1H). ${ }^{13}$ C NMR (125 MHz, DMSO-d6) $\delta$ 161.1, 160.3, 150.7, 141.1, 140.1, 139.7, $135.4,133.8,131.7 \times 2,129.5 \times 2,129.4 \times 2,129.3,128.5,128.4 \times 2,125.5,122.2,121.7$, 120.6, 117.9, 112.8, 111.4, 101.5, 96.8; HRMS m/z $[\mathrm{M}+\mathrm{H}]^{+}$calculated for $\mathrm{C}_{27} \mathrm{H}_{18} \mathrm{NO}_{3}: 404.1281$, found: 404.1271 .

Diethyl 2-amino-4-phenyl-4,7-dihydropyrano[3,2-c]carbazole-3,6-dicarboxylate (6)<smiles>CCOC(=O)c1ccc(O)c2c1[nH]c1ccccc12</smiles>

3aa
1) $\mathrm{PhCHO}$, pyrrolidine, $\mathrm{Et}_{3} \mathrm{~N}$, toluene, $120^{\circ} \mathrm{C}$

2) $\mathrm{NCCH}_{2} \mathrm{COOEt}$, $\mathrm{MeCN}, 50^{\circ} \mathrm{C}$

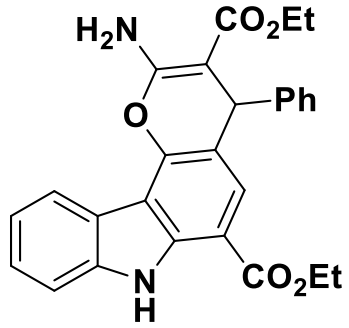

6

A mixture of 3aa $(200 \mathrm{mg}, 0.78 \mathrm{mmol})$, benzaldehyde $(83.1 \mathrm{mg}, 0.78 \mathrm{mmol}$ ), pyrrolidine $(55.6 \mathrm{mg}, 0.78 \mathrm{mmol})$ and triethylamine $(79.2 \mathrm{mg}, 0.78 \mathrm{mmol})$ were stirred in tolune $(5 \mathrm{ml})$ at $120^{\circ} \mathrm{C}$ in an oil bath. After completion of the reaction (TLC), the solvent was washed with brine, dried with anhydrous sodium sulfate, and concentrated to obtain yellow oil which was used directly without other purification. The yellow oil and ethyl 2-cyanoacetate $(88.6 \mathrm{mg}, 0.78 \mathrm{mmol})$ was stirred in acetonitrile $(5 \mathrm{~mL})$ at $50{ }^{\circ} \mathrm{C}$ in an oil bath until completion of the reaction. After completion of the reaction, $15 \mathrm{~mL}$ of water was added to the reaction mixture and extracted with ethylacetate $(3 \times 15 \mathrm{~mL})$. The combined organic layers were dried over anhydrous $\mathrm{Na}_{2} \mathrm{SO}_{4}$, concentrated in vacuo and the residue was purified by column chromatography over silica gel with eluent hexane-ethyl acetate to yield the product. Yield: 42\%. mp 230-232 ${ }^{\circ} \mathrm{C}$; ${ }^{1}$ H NMR (500 MHz, DMSO-d6) $\delta 11.44$ (s, 1H), 8.66 (d, J = 7.8 Hz, 1H), 7.99 (s, 2H), 7.79 (s, 1H), 7.75 (d, J = 8.1 Hz, 1H), 7.48 (t, J = 7.5 $\mathrm{Hz}, 1 \mathrm{H}), 7.32-7.20(\mathrm{~m}, 5 \mathrm{H}), 7.10(\mathrm{t}, \mathrm{J}=6.5 \mathrm{~Hz}, 1 \mathrm{H}), 5.11(\mathrm{~s}, 1 \mathrm{H}), 4.41(\mathrm{~m}, 2 \mathrm{H}), 4.01$ $(\mathrm{m}, 2 \mathrm{H}), 1.36(\mathrm{t}, \mathrm{J}=7.0 \mathrm{~Hz}, 3 \mathrm{H}), 1.11(\mathrm{t}, \mathrm{J}=7.0 \mathrm{~Hz}, 3 \mathrm{H}) .{ }^{13} \mathrm{C}$ NMR (125 MHz, DMSO-d6) $\delta 170.1,167.1,162.1,150.6,149.2,142.0,141.0,130.4,130.2 \times 2$, $129.1 \times 2,128.1,127.8,125.1,121.7,121.6,117.8,113.9,113.1,110.5,78.7,62.4$, 
60.6, 39.6, 16.3, 16.1; HRMS m/z $[\mathrm{M}+\mathrm{H}]^{+}$calculated for $\mathrm{C}_{27} \mathrm{H}_{25} \mathrm{~N}_{2} \mathrm{O}_{5}: 457.1758$, found: 457.1760 .

\section{Crystal Data of 3aa}

Single crystal of 3aa was obtained by slowly diffusing acetone vapor into the atmosphere. X-ray diffractions of all single crystals were carried out at 100(2) K on a 'Bruker APEX-II CCD' diffractometer using Mo-K $\alpha$ radiation $(\lambda=0.71073 \AA)$. X-ray crystallographic data of 3aa were solutions at Temperature $=140.0 \mathrm{~K}$. Empirical formula: $\mathrm{C}_{17} \mathrm{H}_{19} \mathrm{NO}_{4} \mathrm{~S}$, formula weight $=333.39$, crystal system: orthorhombic, space group: $\quad \mathrm{P} 2{ }_{1} 2{ }_{1}{ }_{1}, \mathrm{a}=6.0036(2) \AA, \mathrm{b}=16.1582(7) \AA, \mathrm{c}=16.6086(7) \AA, \alpha=90^{\circ}, \beta=$ $90^{\circ}, \gamma=90^{\circ}$, volume $=1611.16(11) \AA^{3}, Z=4$. Displacement ellipsoids are drawn at the $50 \%$ probability level.

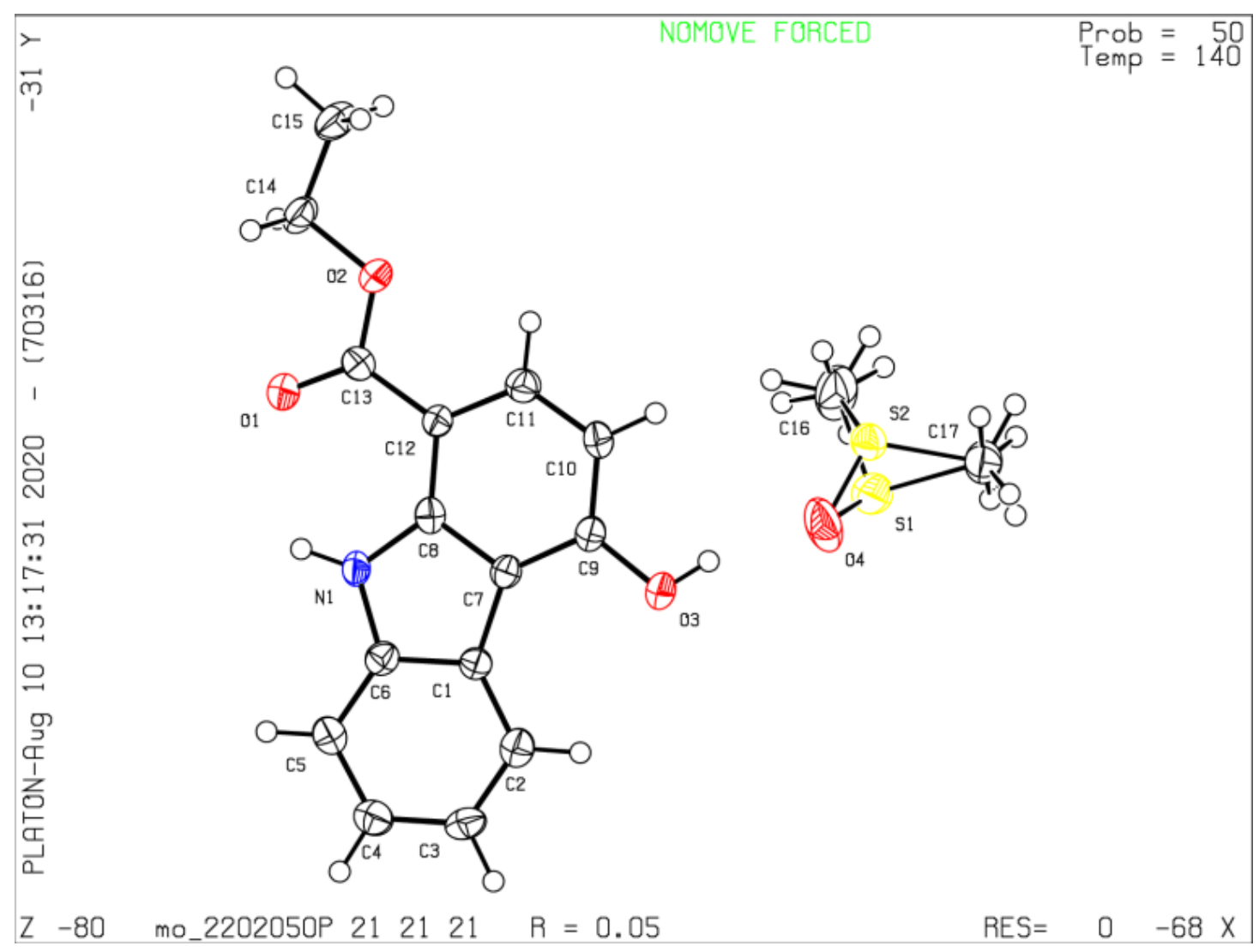




\section{Reference}

(1)Borah, A. J.;Shi, Z. Chem. Commun. 2017, 53, 3945.

(2)Diab, S.;Abdelaziz, A. M.;Li, P.;Teo, T.;Basnet, S. K. C.;Noll, B.;Rahaman, M. H.;Lu, J.;Hou, J.;Yu, M.;Le, B. T.;Albrecht, H.;Milne, R. W.;Wang, S. Eur. J. Med. Chem. 2017, 139, 762. 


\section{NMR Spectra}

${ }^{1}$ H NMR (600 MHz, DMSO-d6)
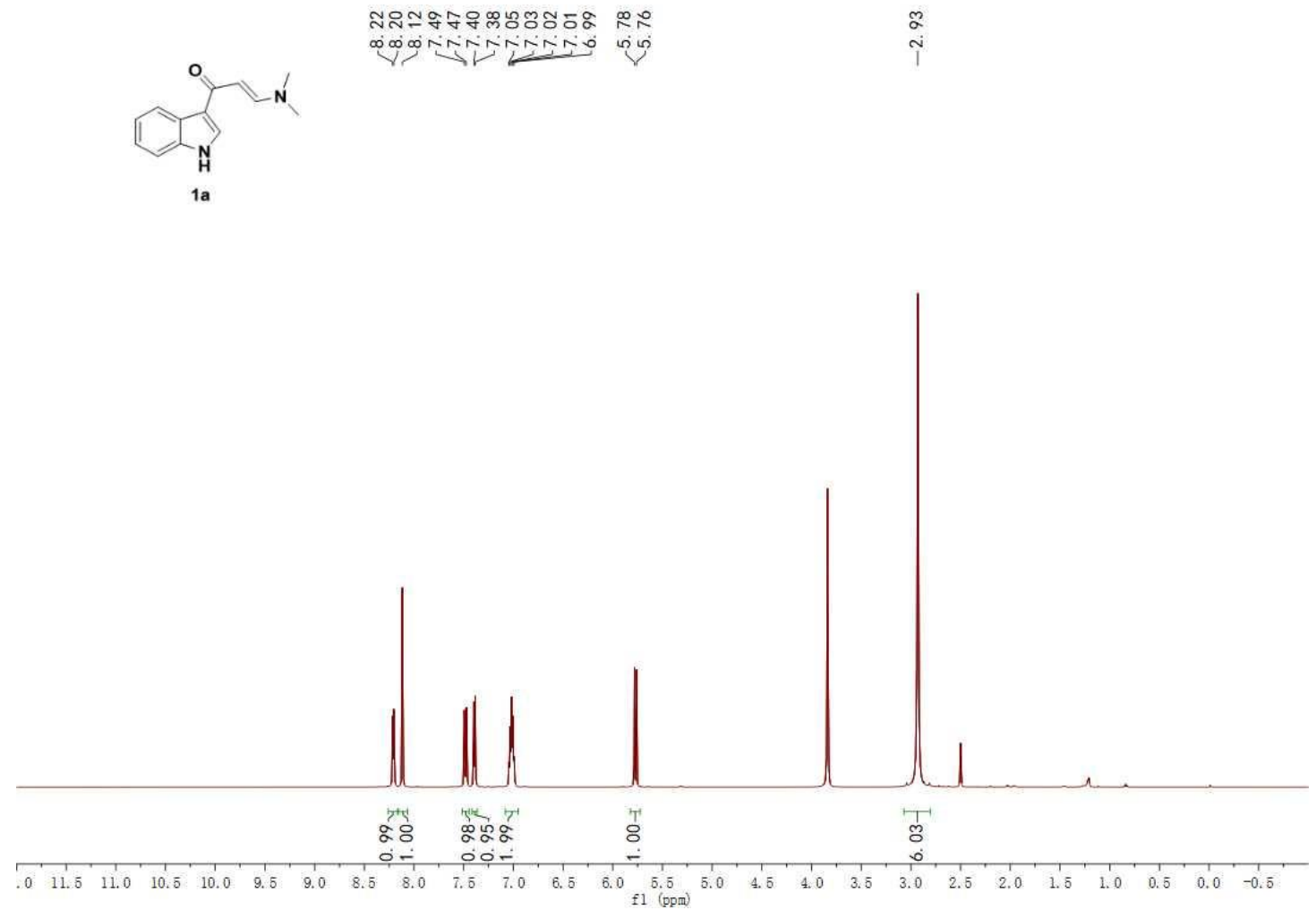

${ }^{13}$ C NMR (150 MHz, DMSO-d6)
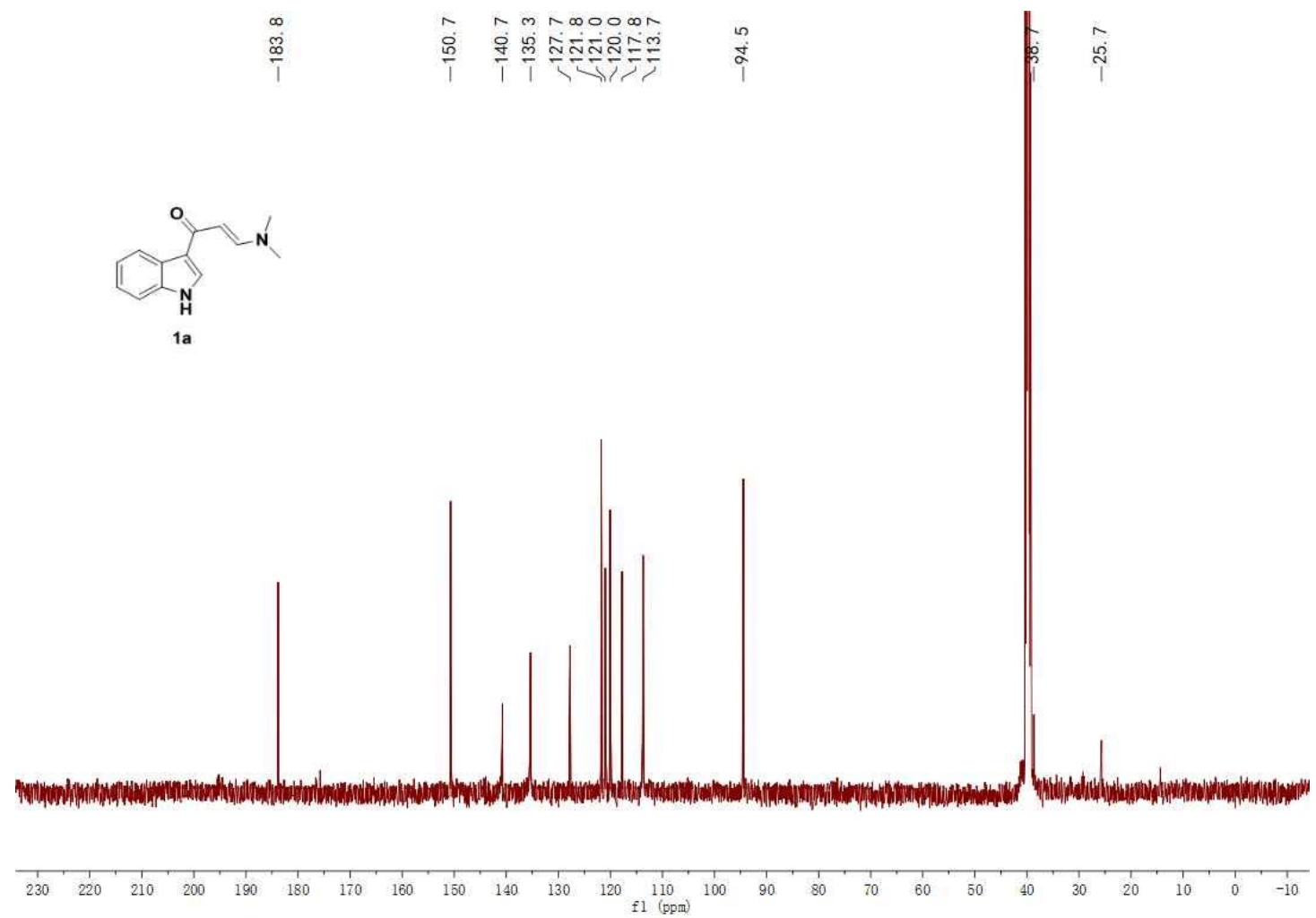
${ }^{1}$ H NMR (500 MHz, DMSO-d6)

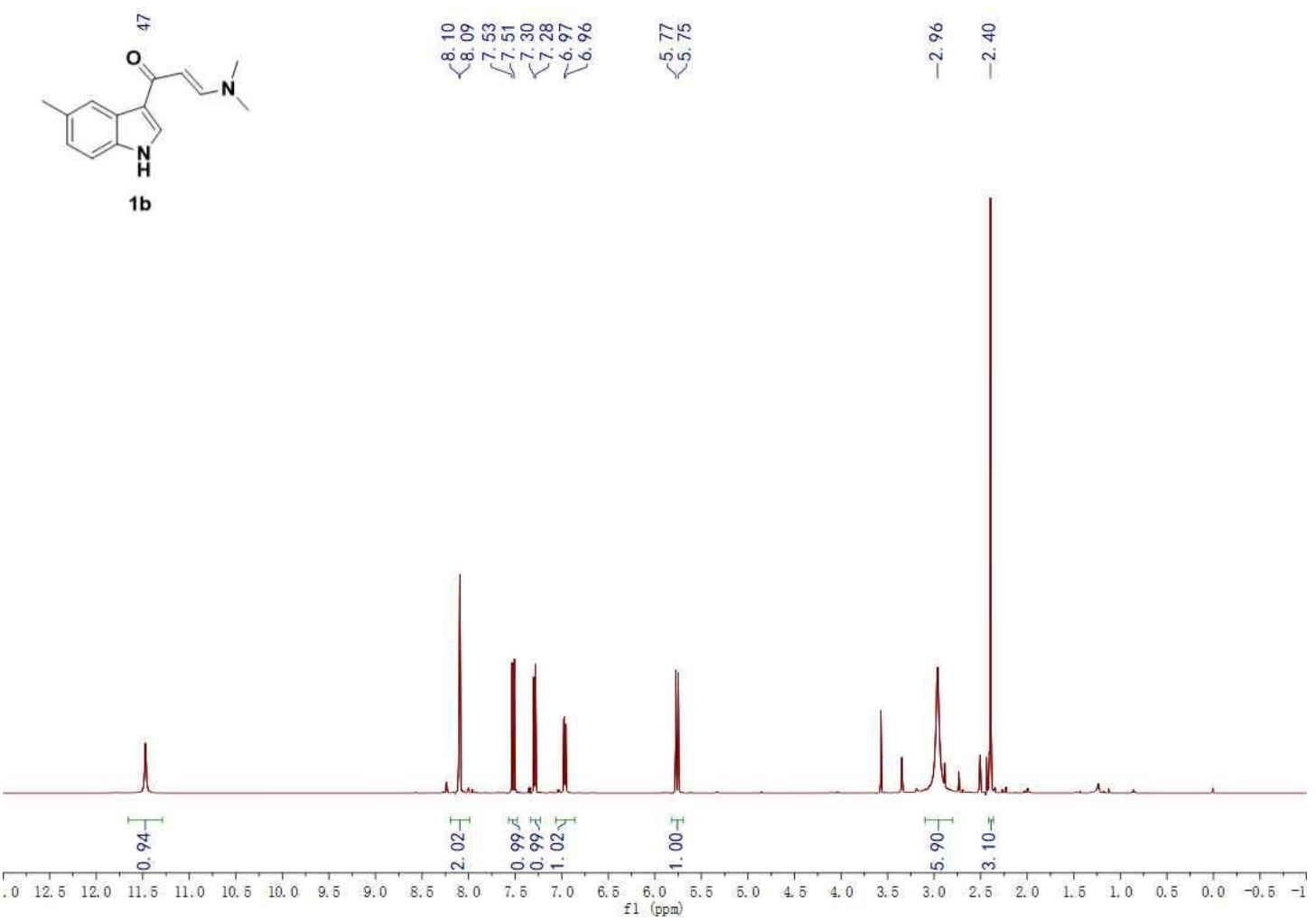

${ }^{13}$ C NMR (125 MHz, DMSO-d6)
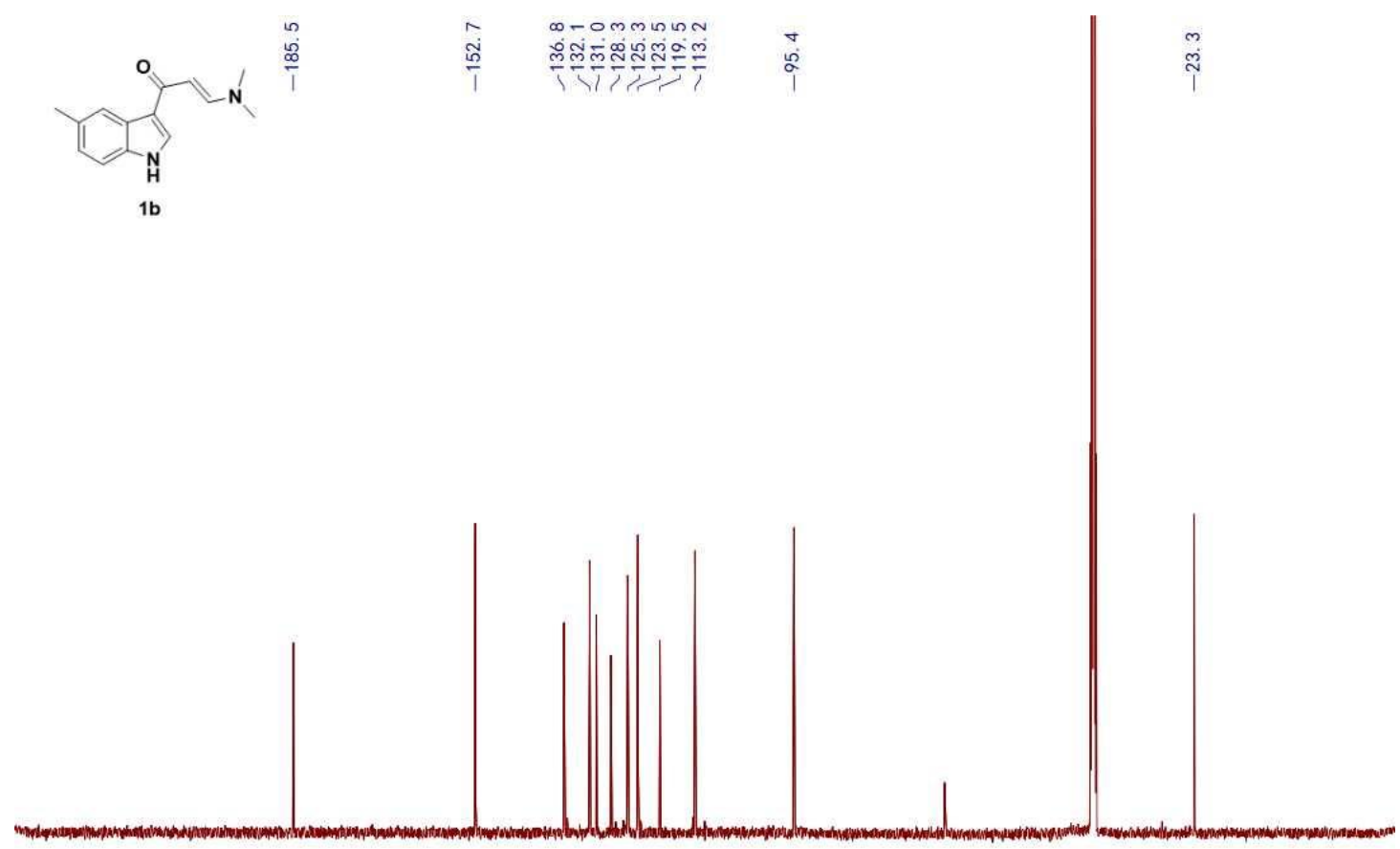

$\begin{array}{llllllllllllllllllllllllllllllllll}230 & 220 & 210 & 200 & 190 & 180 & 170 & 160 & 150 & 140 & 130 & 120 & 110 & 100 & 90 & 80 & 70 & 60 & 50 & 40 & 30 & 20 & 10 & 0 & -10\end{array}$ 
${ }^{1}$ H NMR (500 MHz, DMSO-d6)

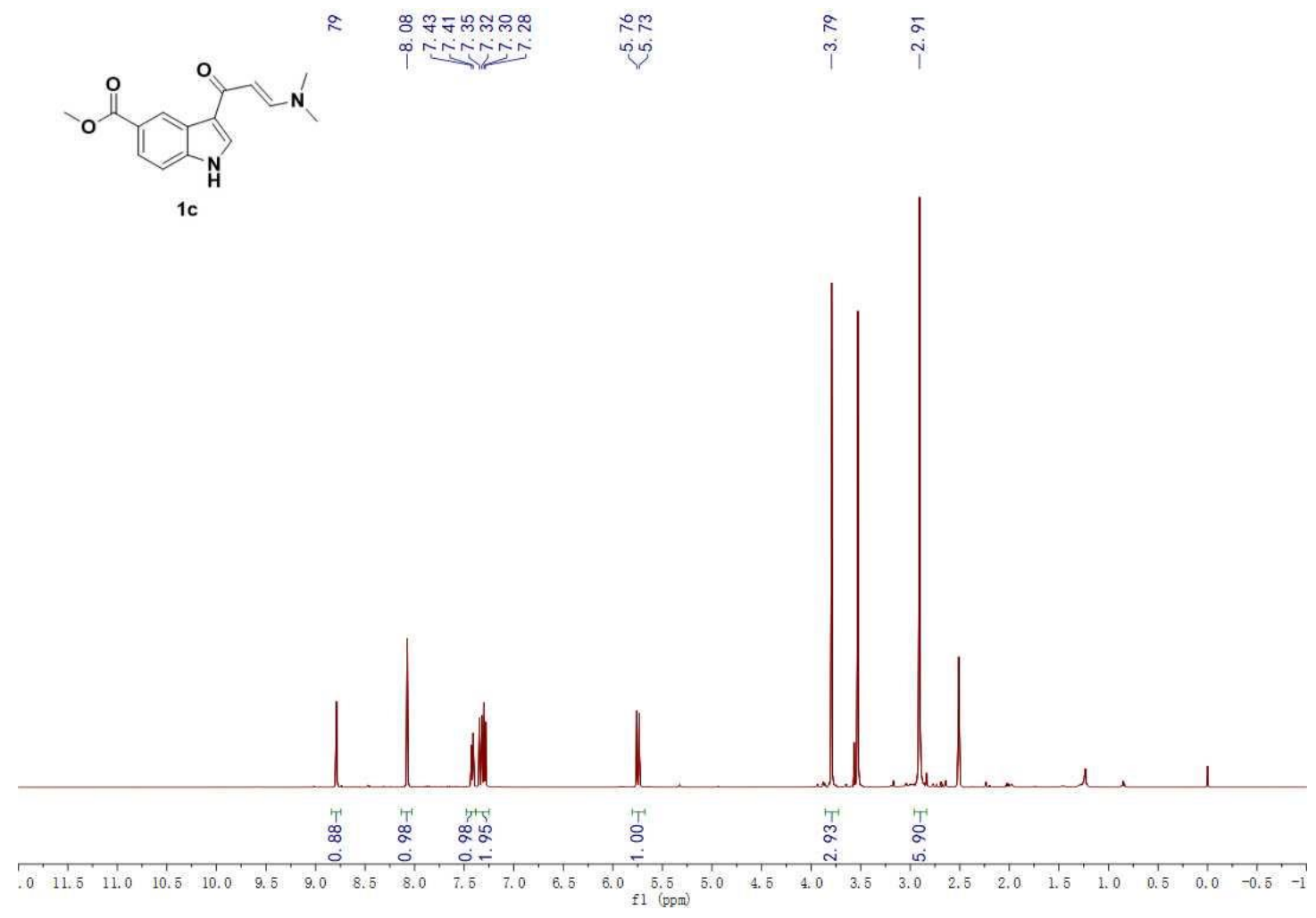

${ }^{13}$ C NMR (125 MHz, DMSO-d6)

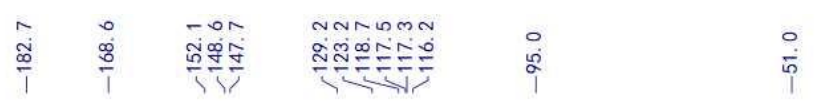

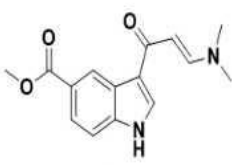

1c

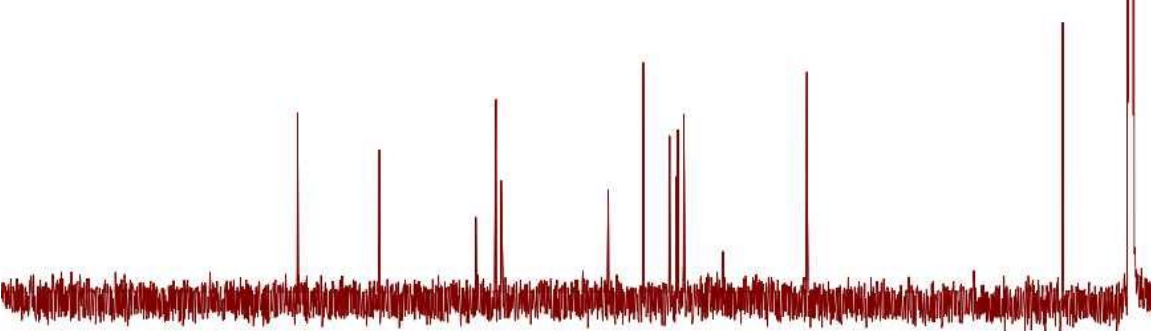

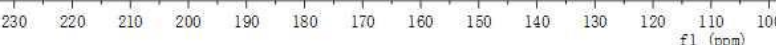


${ }^{1}$ H NMR (600 MHz, DMSO-d6)

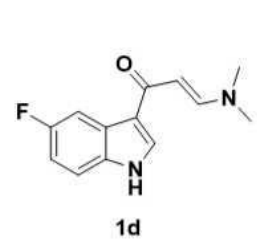

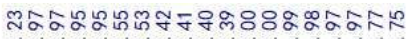

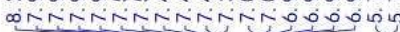

$\hat{\mathrm{a}}$

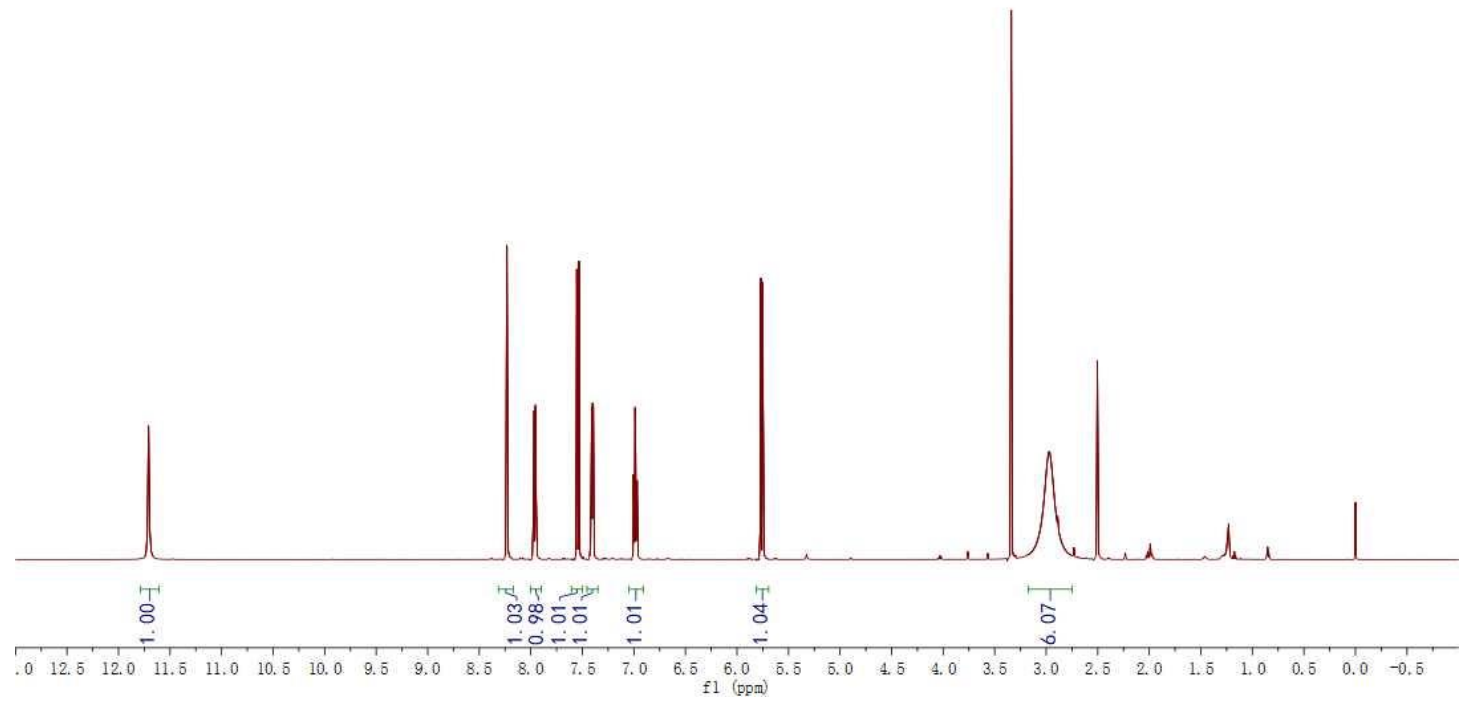

${ }^{13}$ C NMR (150 MHz, DMSO-d6)
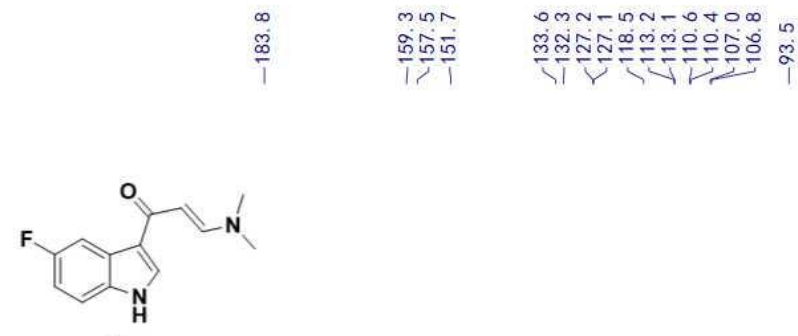

1d

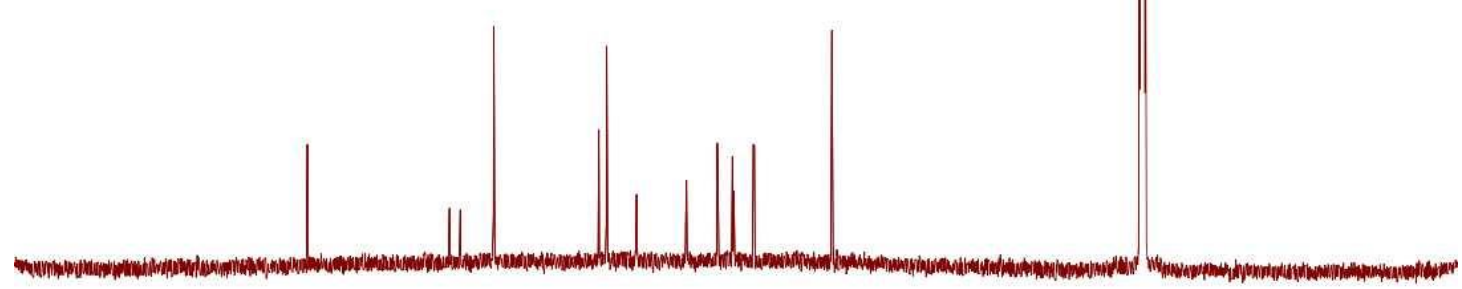

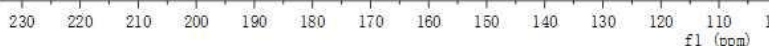


${ }^{1}$ H NMR (500 MHz, DMSO-d6)

$\stackrel{\infty}{=}$

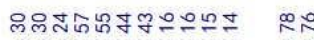

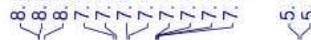

$\stackrel{\infty}{i}$
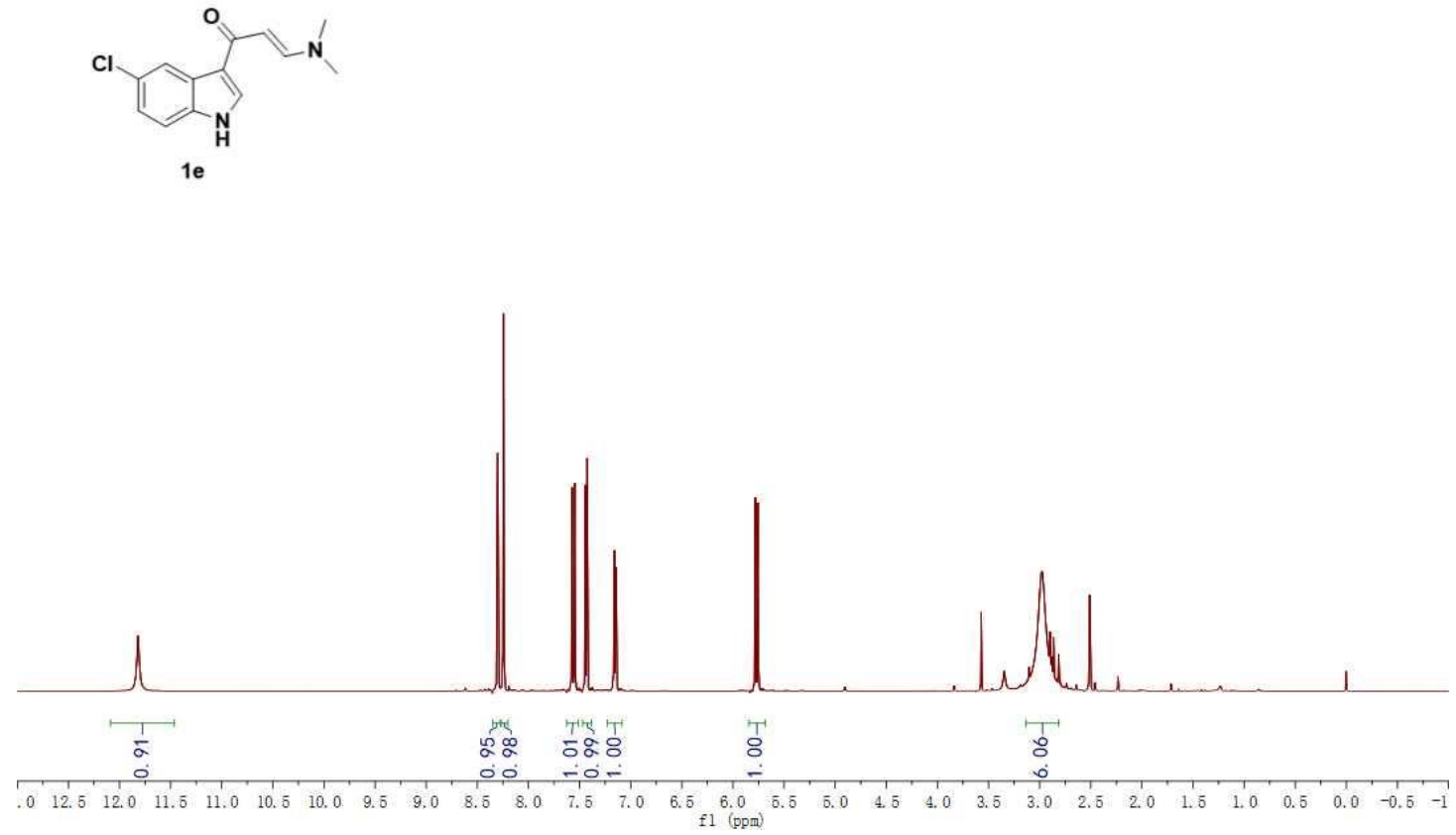

${ }^{13}$ C NMR (125 MHz, DMSO-d6)
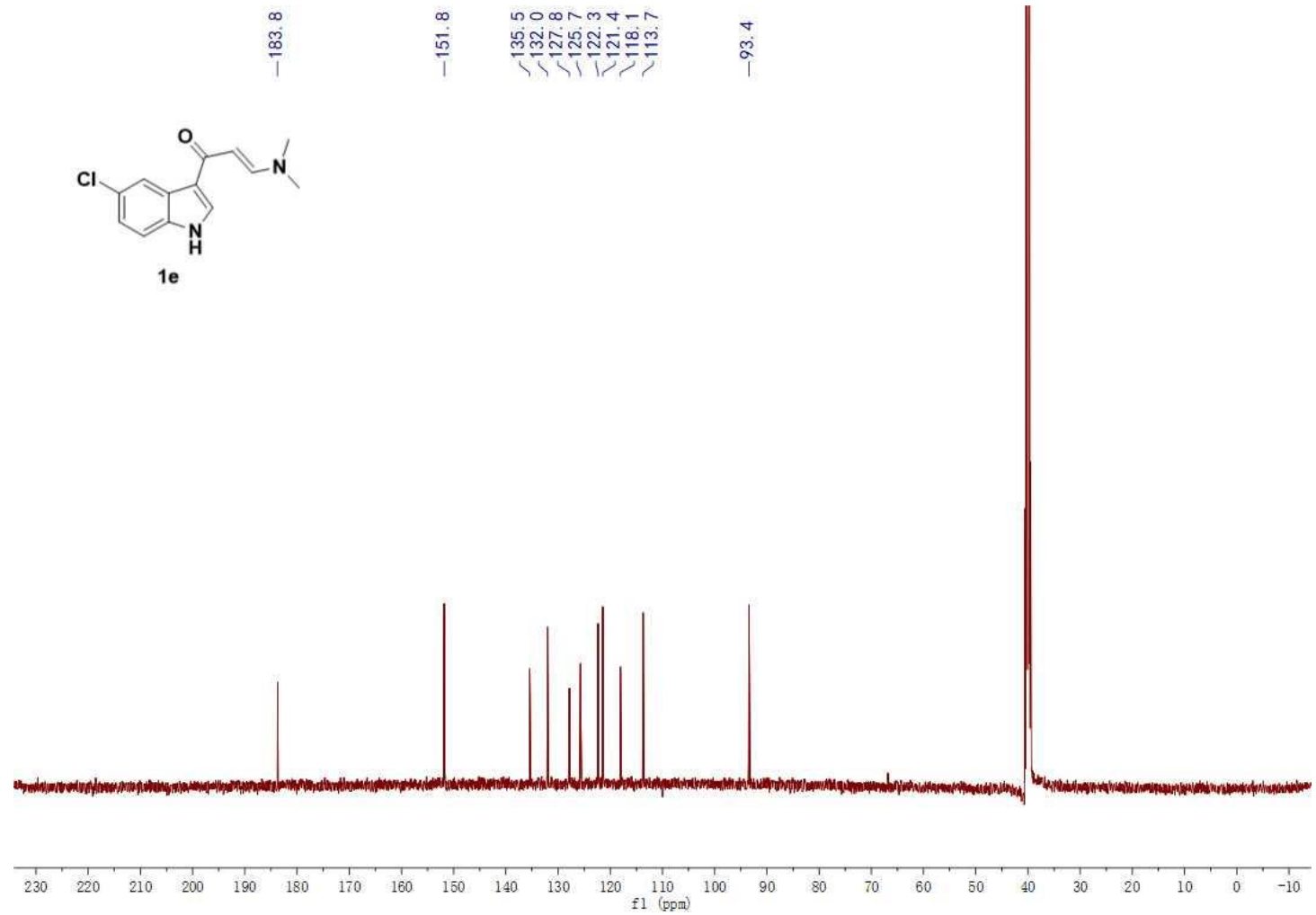
${ }^{1}$ H NMR (500 MHz, DMSO-d6)

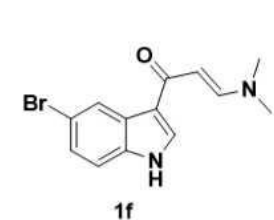

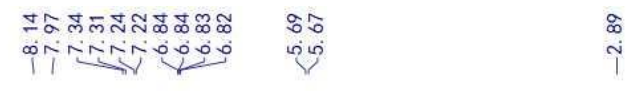

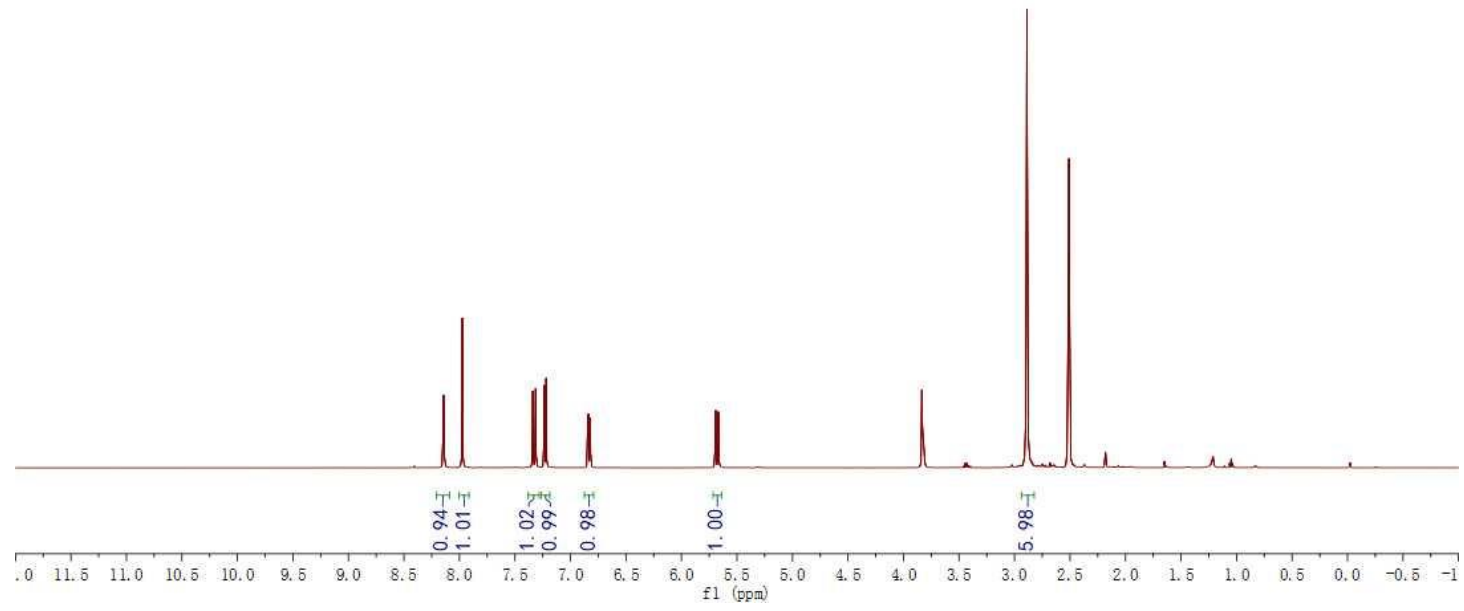

${ }^{13}$ C NMR (125 MHz, DMSO-d6)
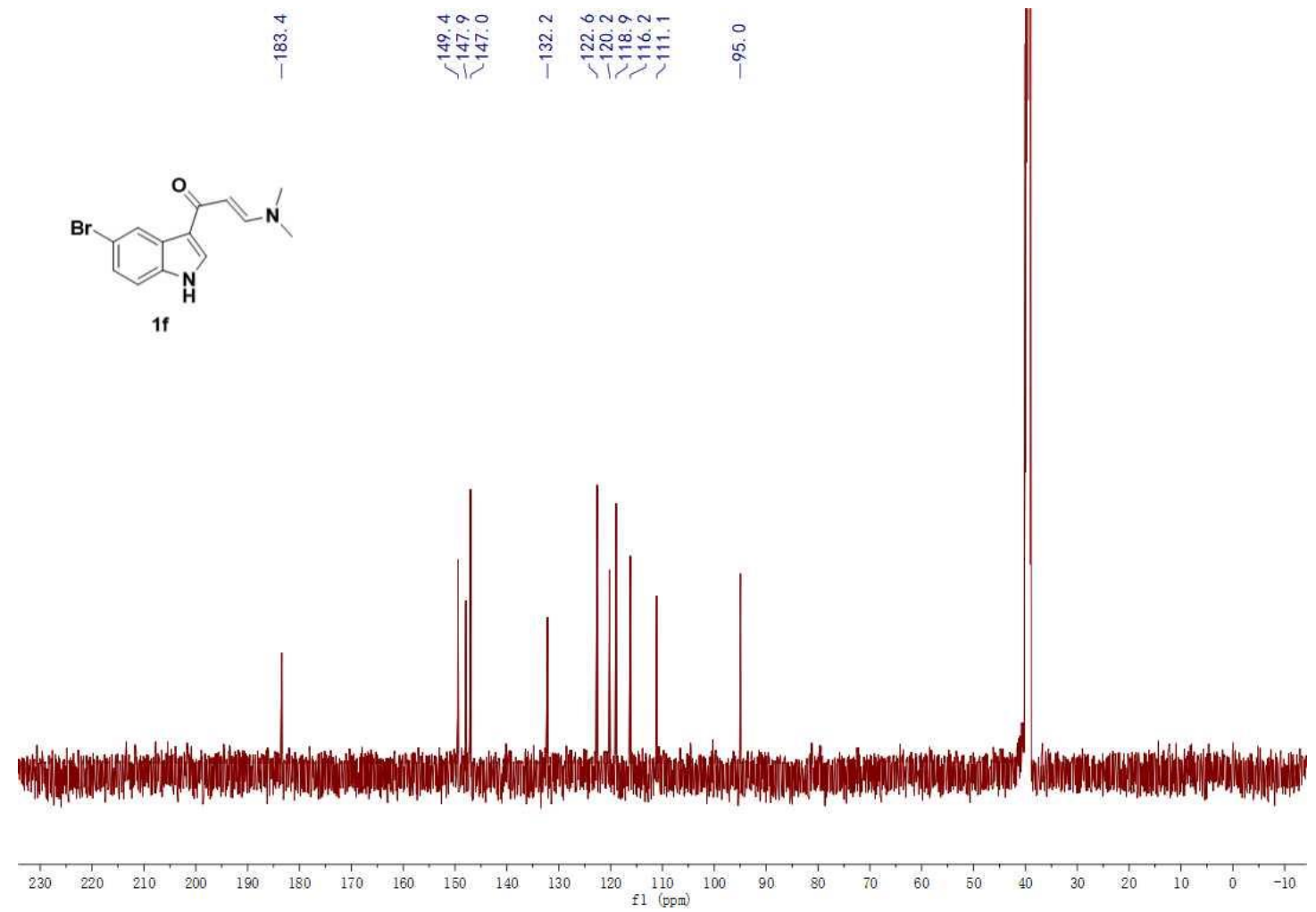
${ }^{1}$ H NMR (500 MHz, DMSO-d6)

$$
\stackrel{\gtrless}{i}
$$
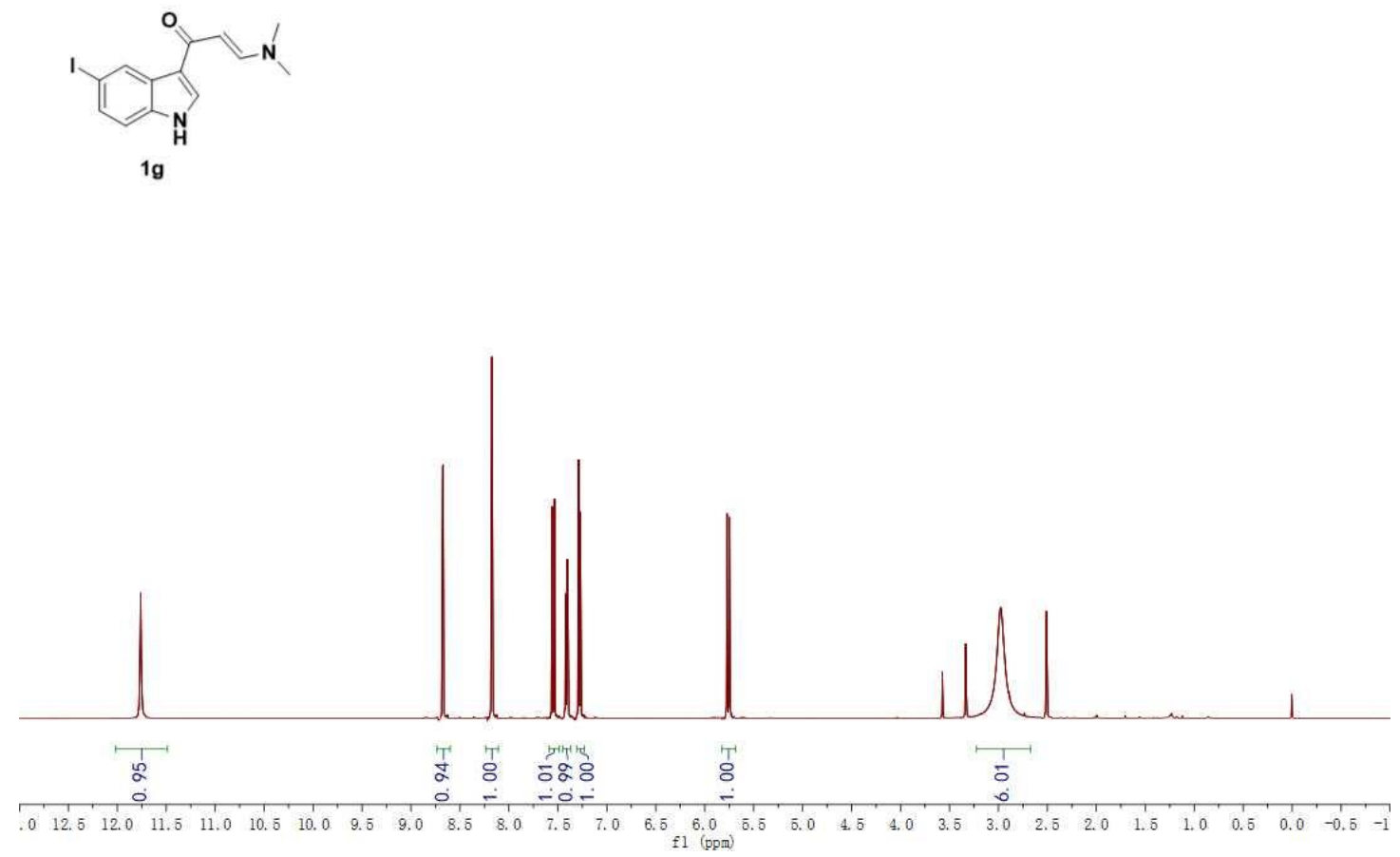

${ }^{13}$ C NMR (125 MHz, DMSO-d6)
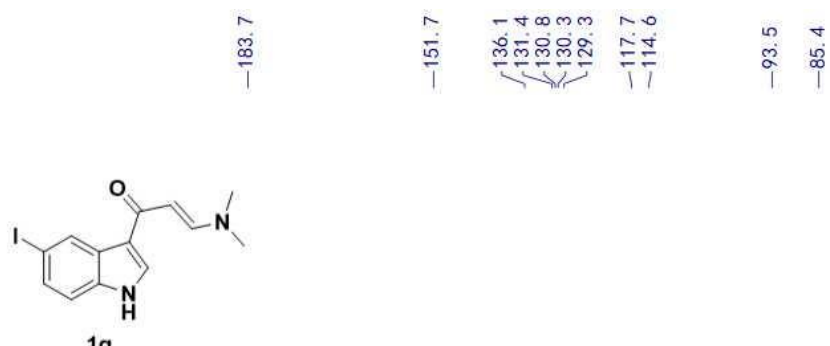

$1 \mathrm{~g}$

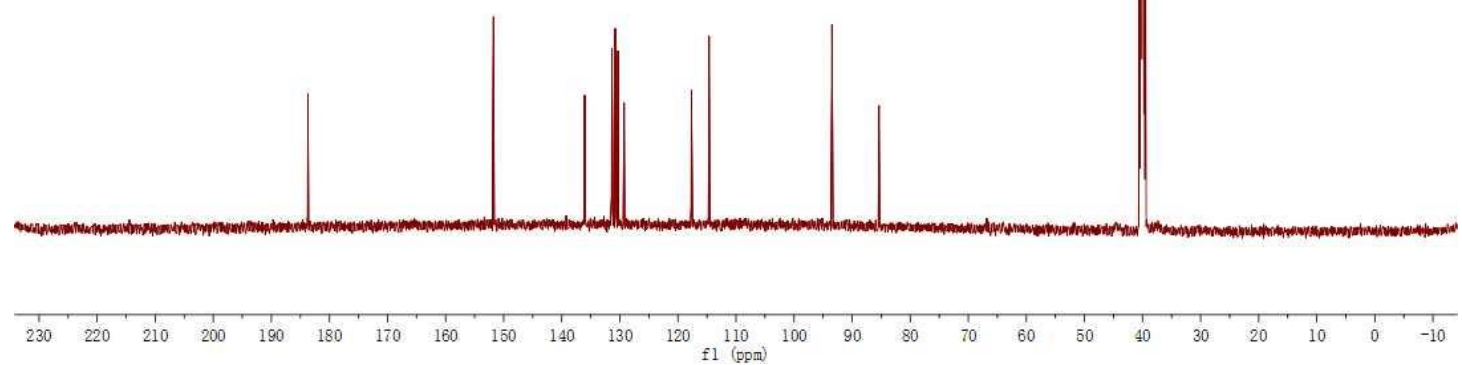


${ }^{1}$ H NMR (500 MHz, DMSO-d6)
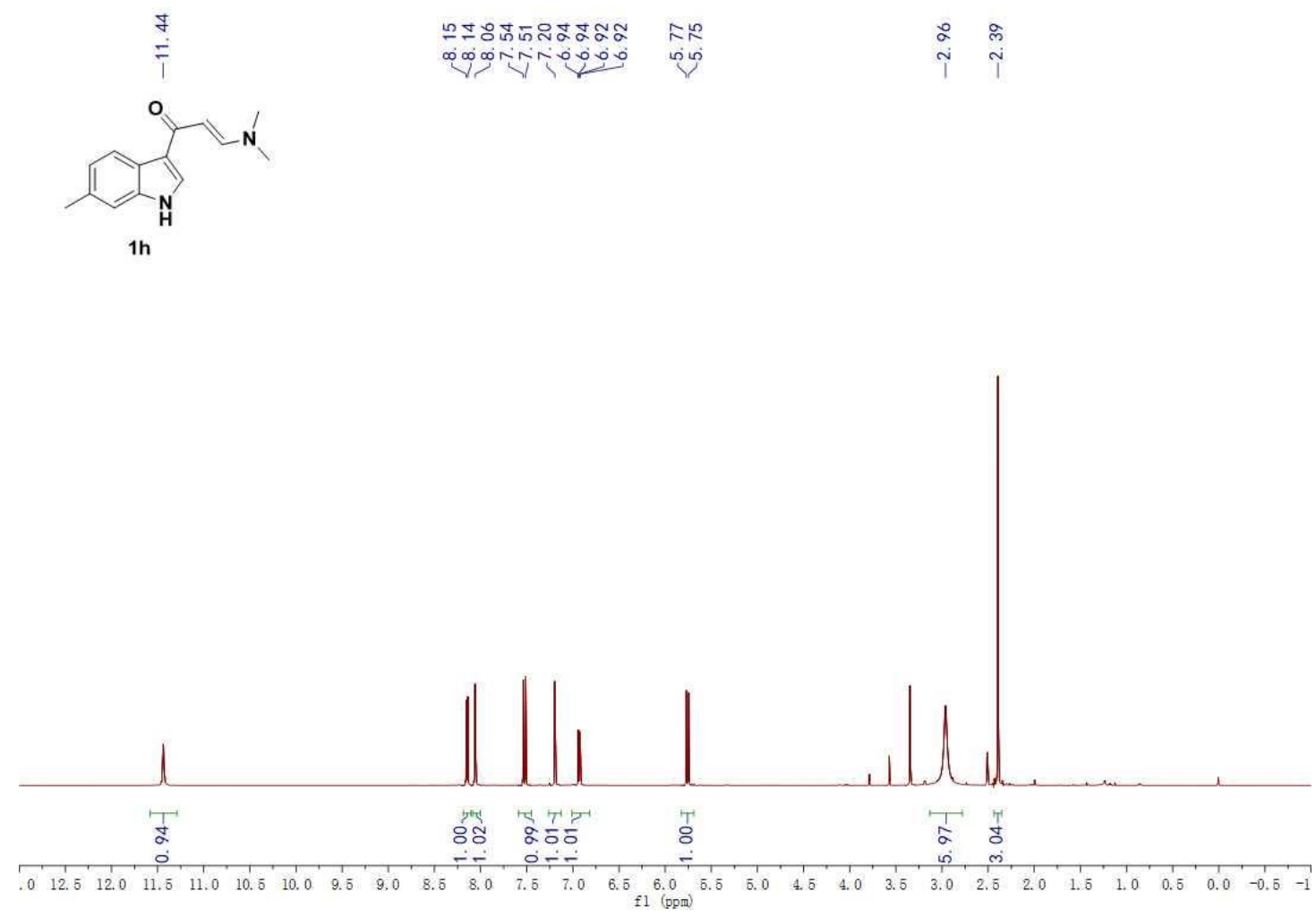

${ }^{13}$ C NMR (125 MHz, DMSO-d6)
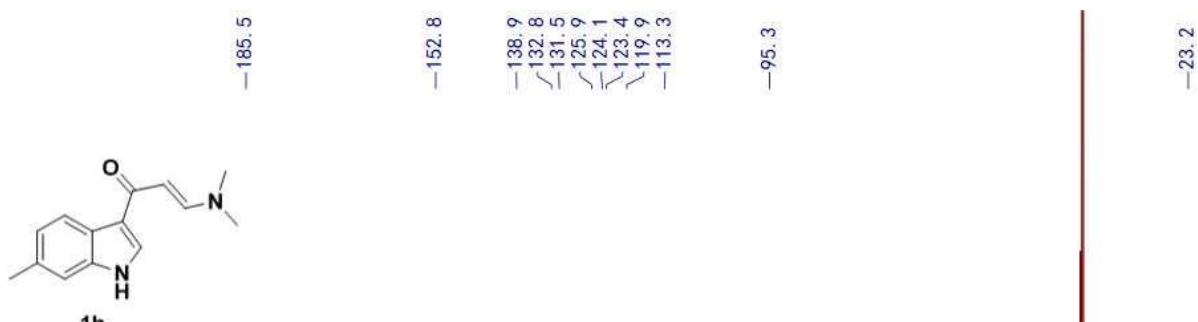

1h

$\stackrel{\text { ก }}{\text { ก }}$

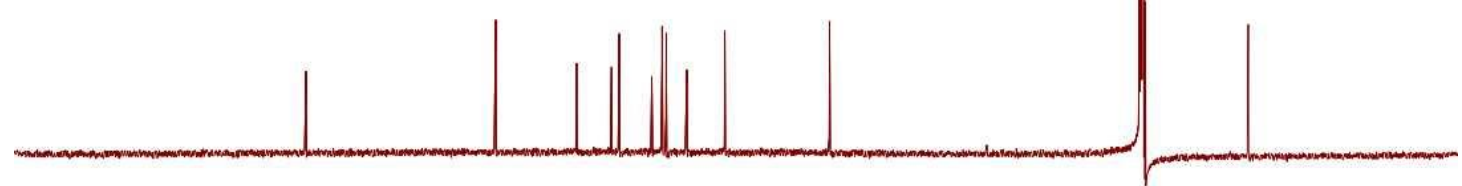

$\begin{array}{llllllllllllllllllllllllllllllllllll}230 & 220 & 210 & 200 & 190 & 180 & 170 & 160 & 150 & 140 & 130 & 120 & 110 & 100 & 90 & 80 & 70 & 60 & 50 & 40 & 30 & 20 & 10 & 0 & -10\end{array}$ 
${ }^{1}$ H NMR (500 MHz, DMSO-d6)

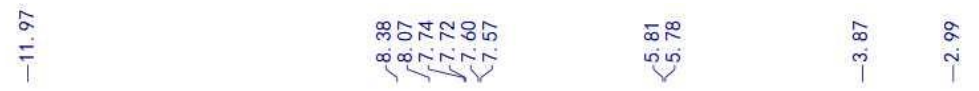<smiles>COC(=O)c1ccc2c(C(=O)C=CN(C)C)c[nH]c2c1</smiles>

$1 \mathrm{i}$

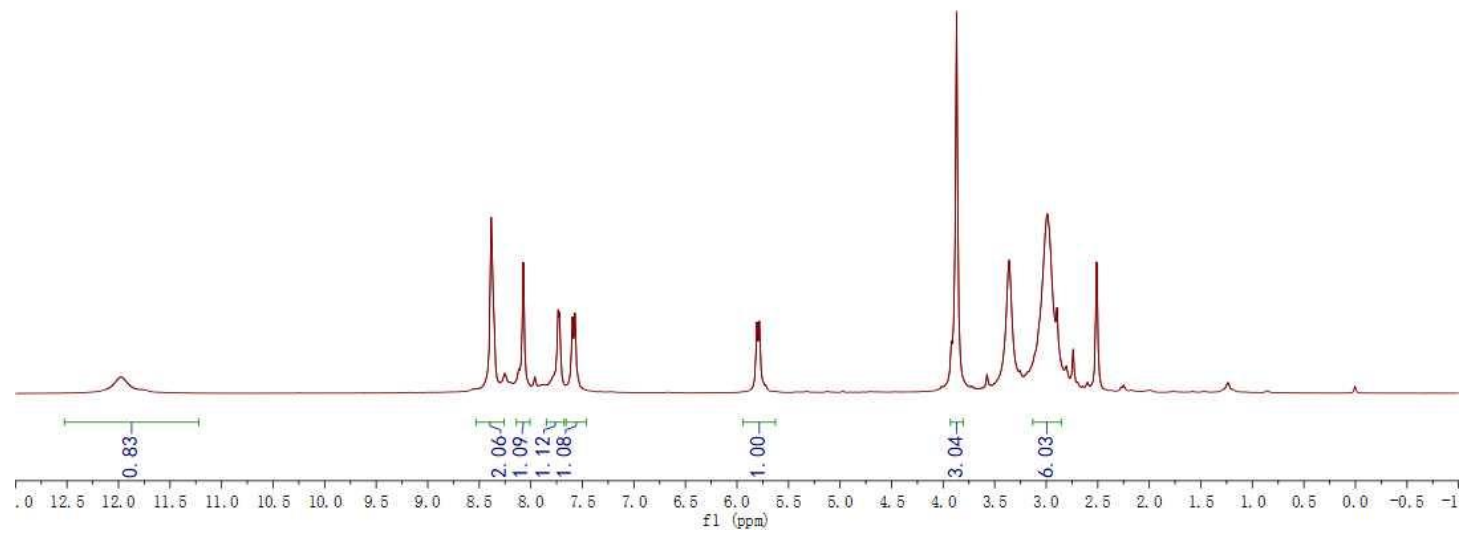

${ }^{13}$ C NMR (125 MHz, DMSO-d6)

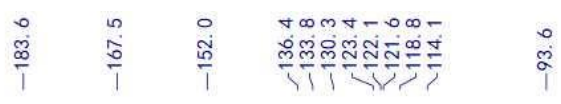

ֻึं<smiles>COC(=O)c1ccc2c(C(=O)C=NC(C)C)c[nH]c2c1</smiles>

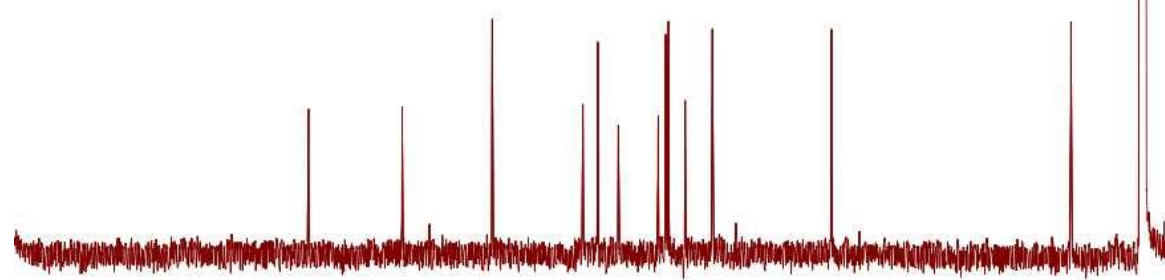

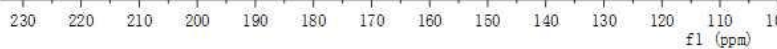


${ }^{1}$ H NMR (500 MHz, DMSO-d6)
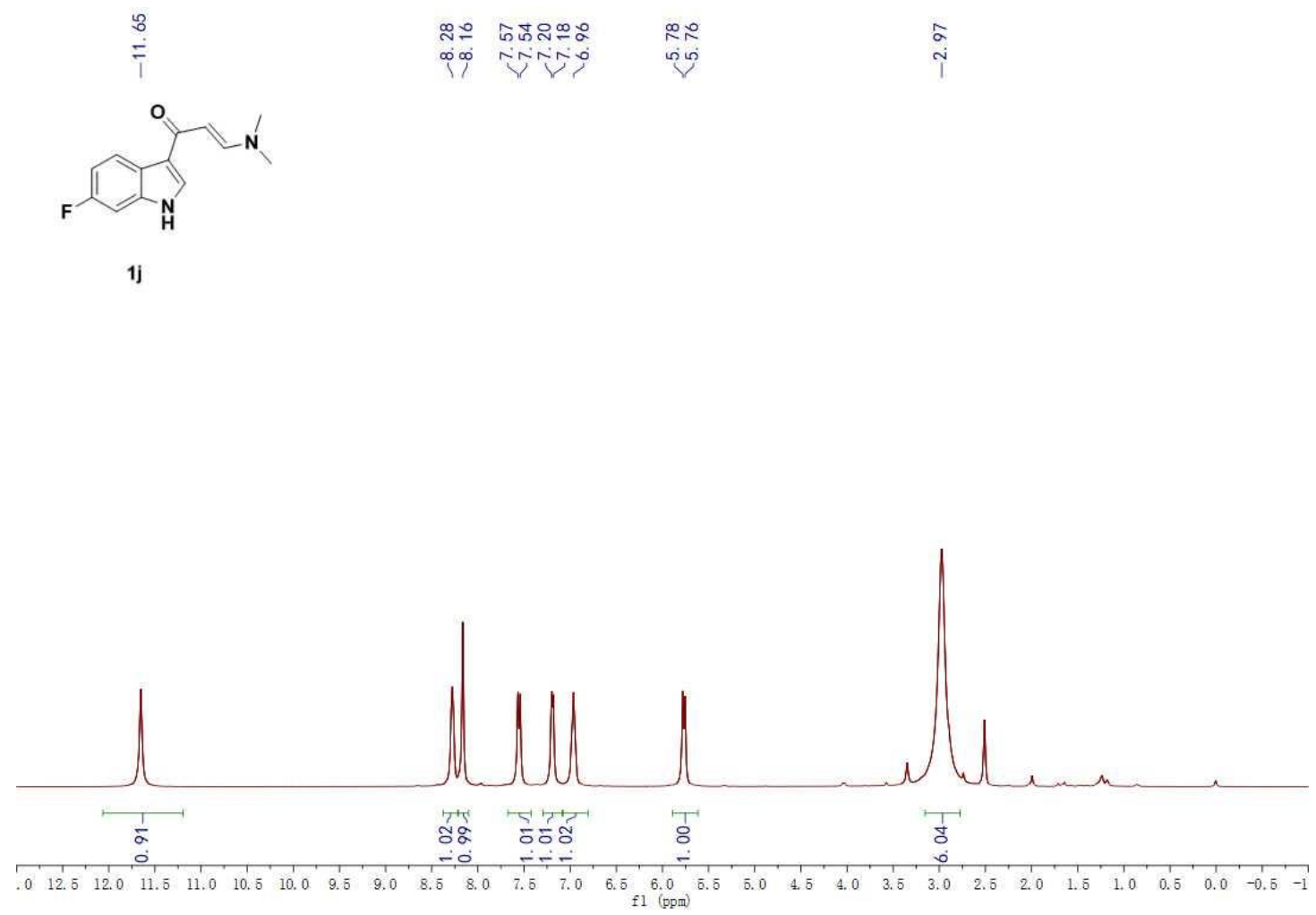

${ }^{13}$ C NMR (125 MHz, DMSO-d6)
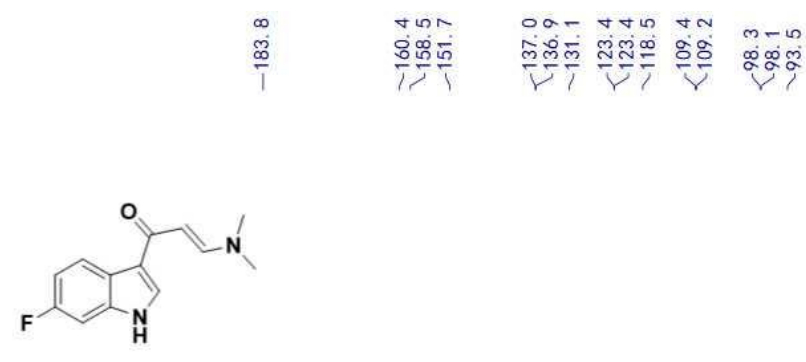

$1 \mathrm{j}$

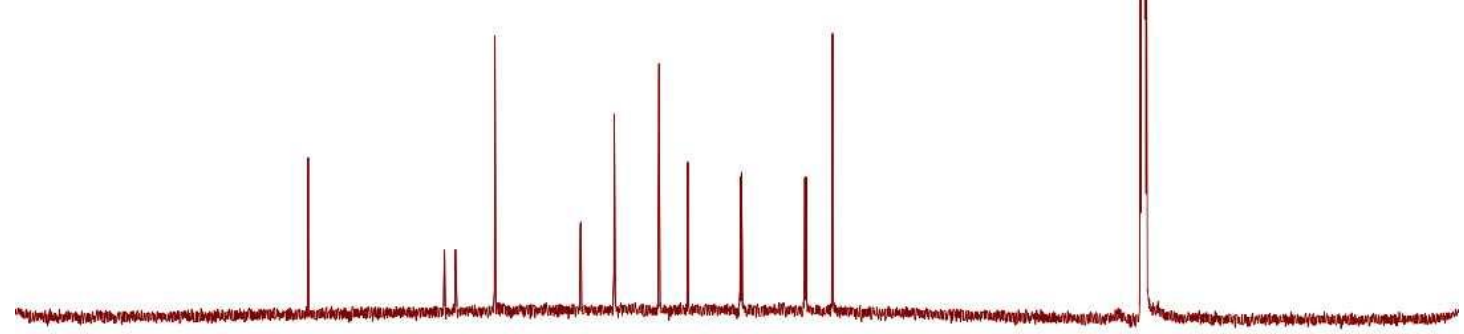

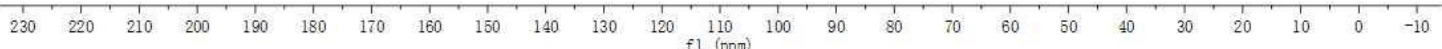


${ }^{1}$ H NMR (500 MHz, DMSO-d6)

$\stackrel{?}{i}$

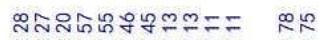

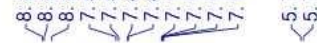

จิ<smiles>CN(C)/C=C/C(=O)c1c[nH]c2cc(Cl)ccc12</smiles>

$1 \mathrm{k}$

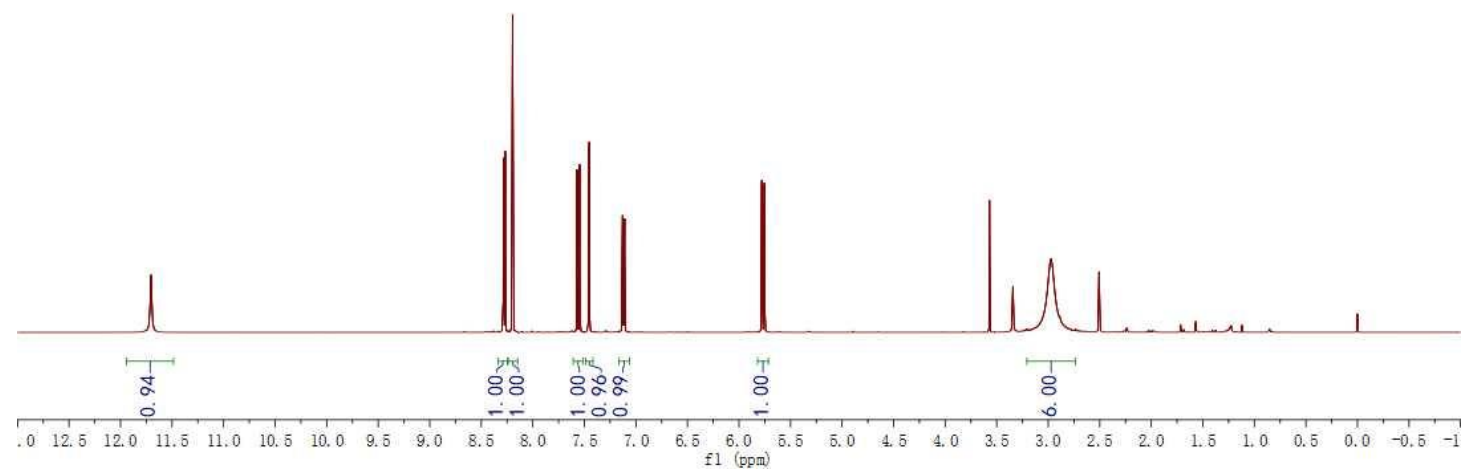

${ }^{13}$ C NMR (125 MHz, DMSO-d6)
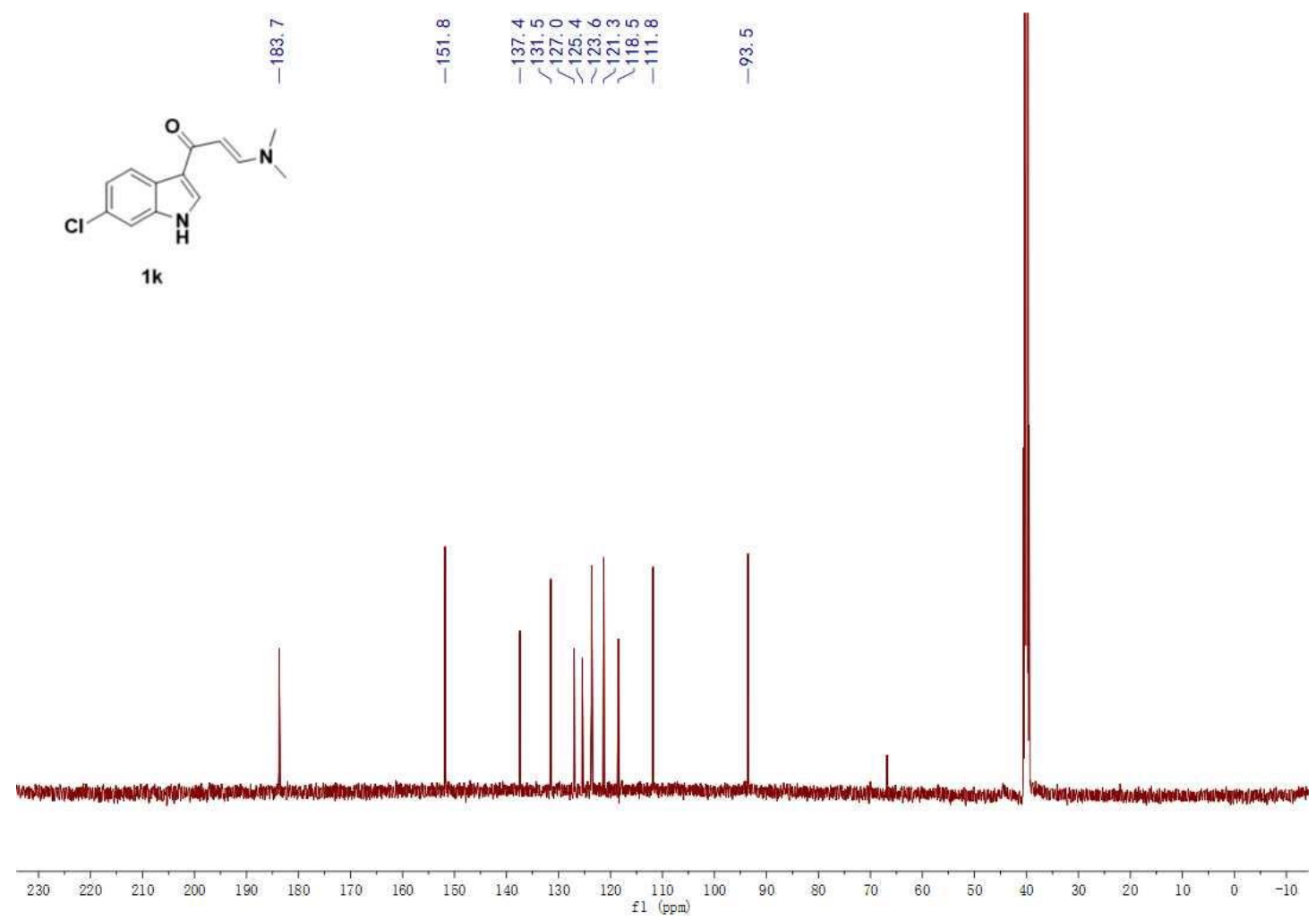
${ }^{1}$ H NMR (500 MHz, DMSO-d6)

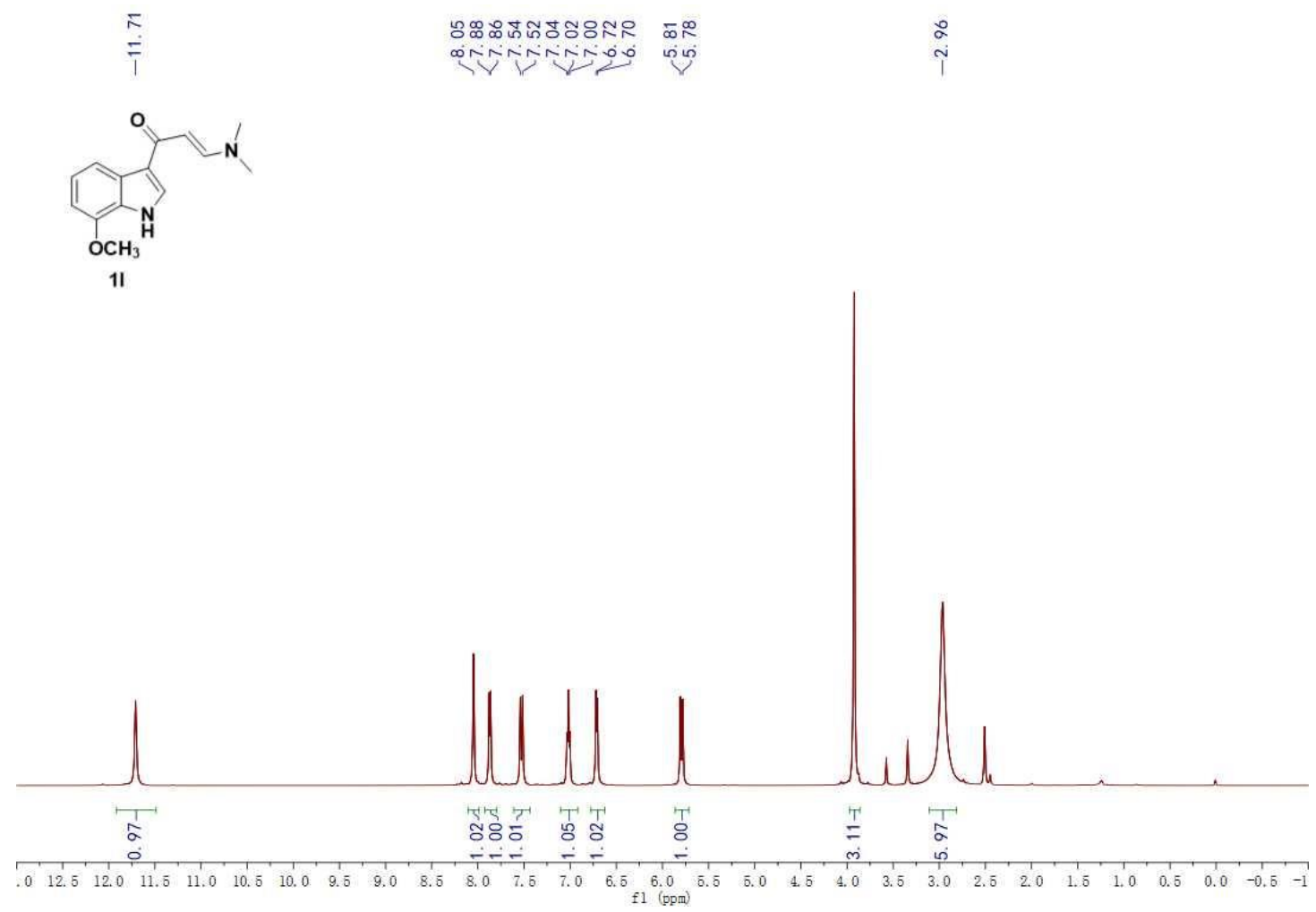

${ }^{13}$ C NMR (125 MHz, DMSO-d6)

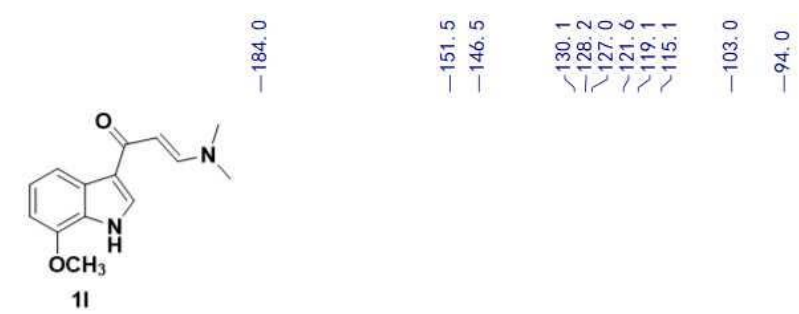

$\stackrel{\circ}{\stackrel{\circ}{0}}$

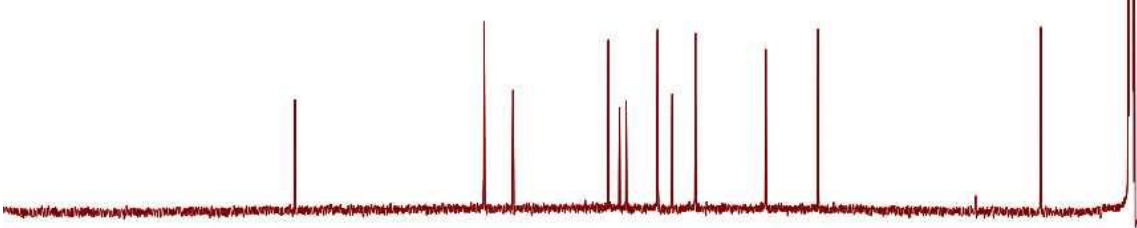

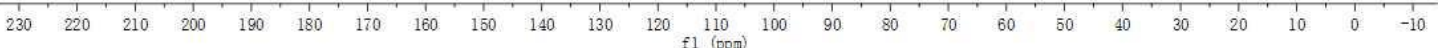


${ }^{1}$ H NMR (500 MHz, DMSO-d6)

$\frac{}{1}$

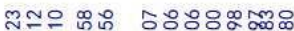

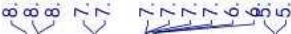

$\stackrel{\infty}{i}$

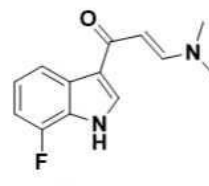

$1 \mathrm{~m}$

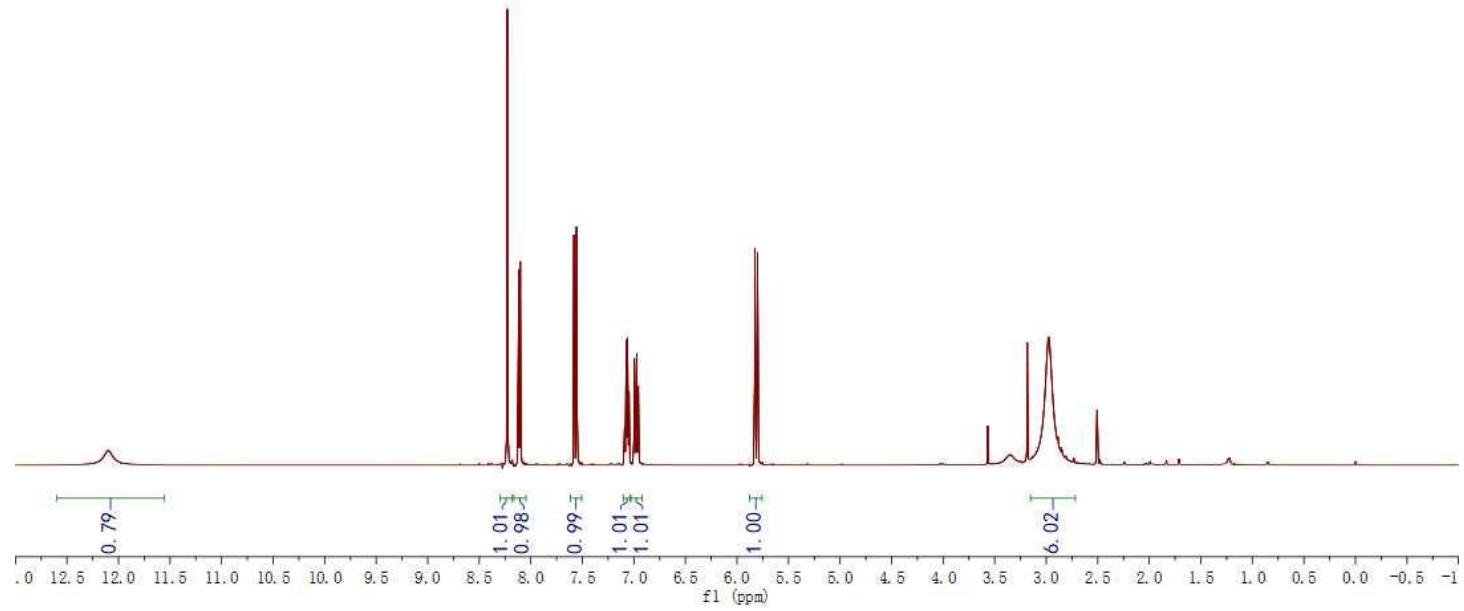

${ }^{13}$ C NMR (125 MHz, DMSO-d6)
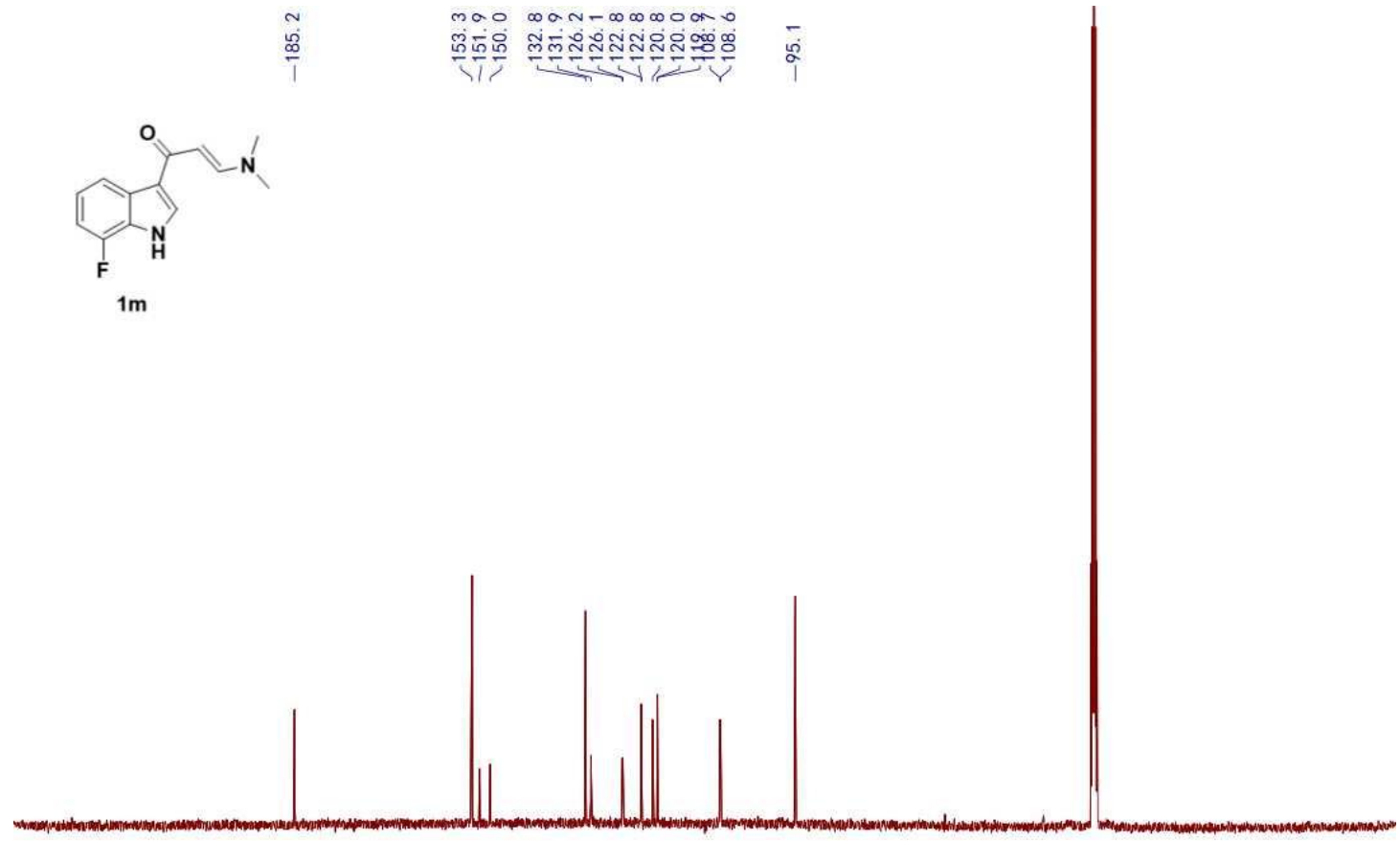

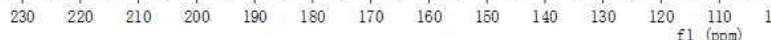


${ }^{1}$ H NMR (500 MHz, DMSO-d6)

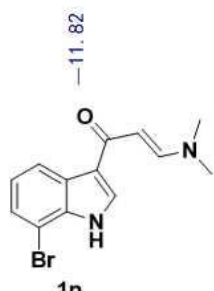

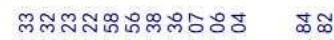

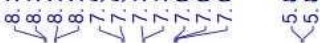

$\stackrel{4}{2}$

1n

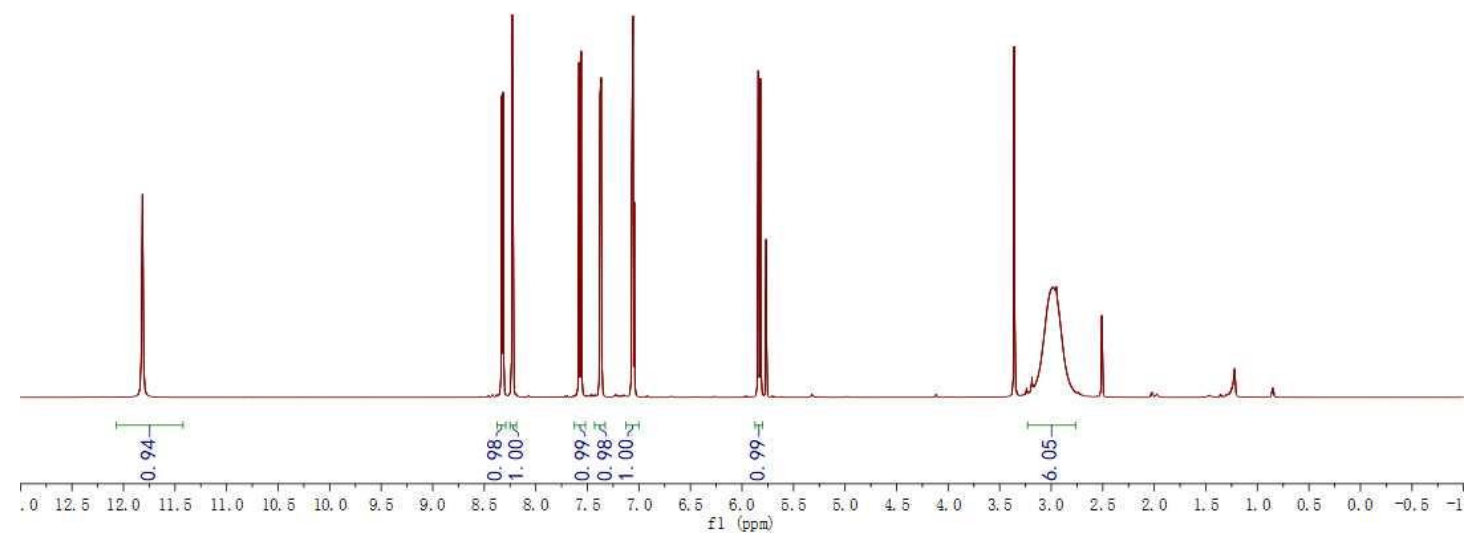

${ }^{13}$ C NMR (125 MHz, DMSO-d6)
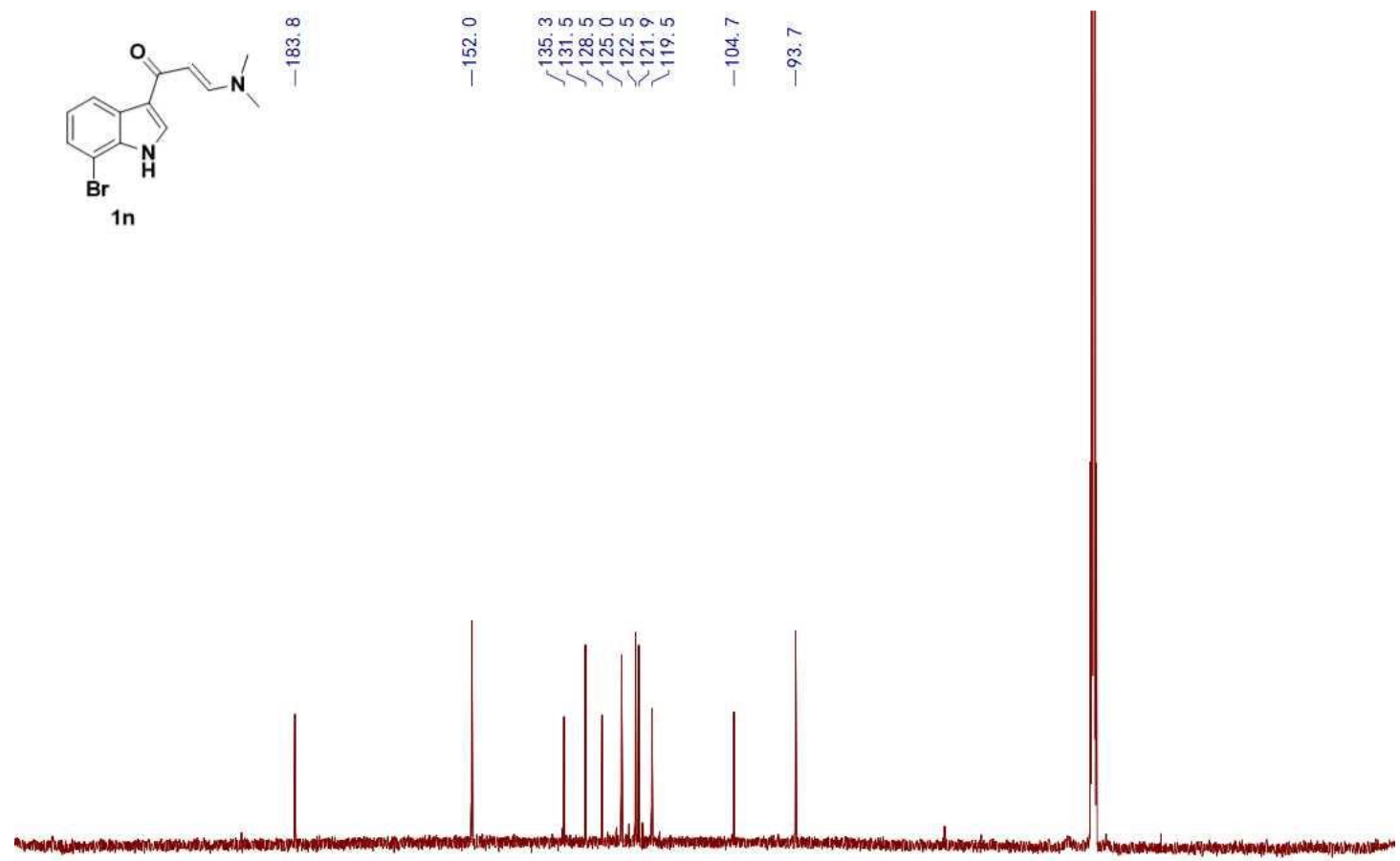

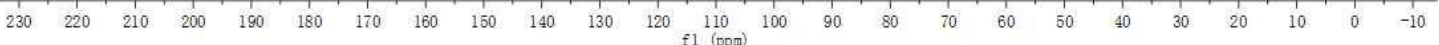


${ }^{1}$ H NMR (500 MHz, DMSO-d6)
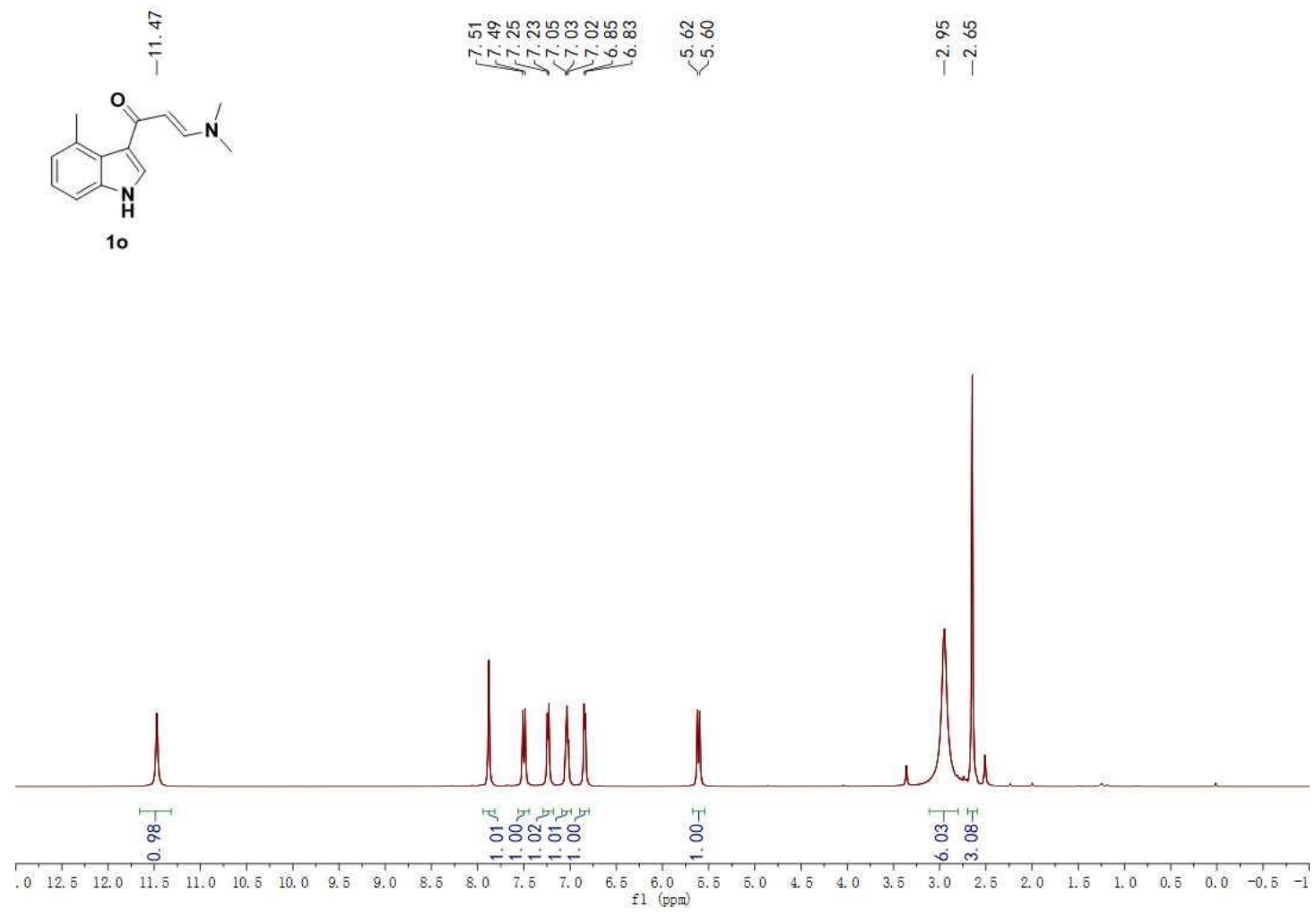

${ }^{13}$ C NMR (125 MHz, DMSO-d6)
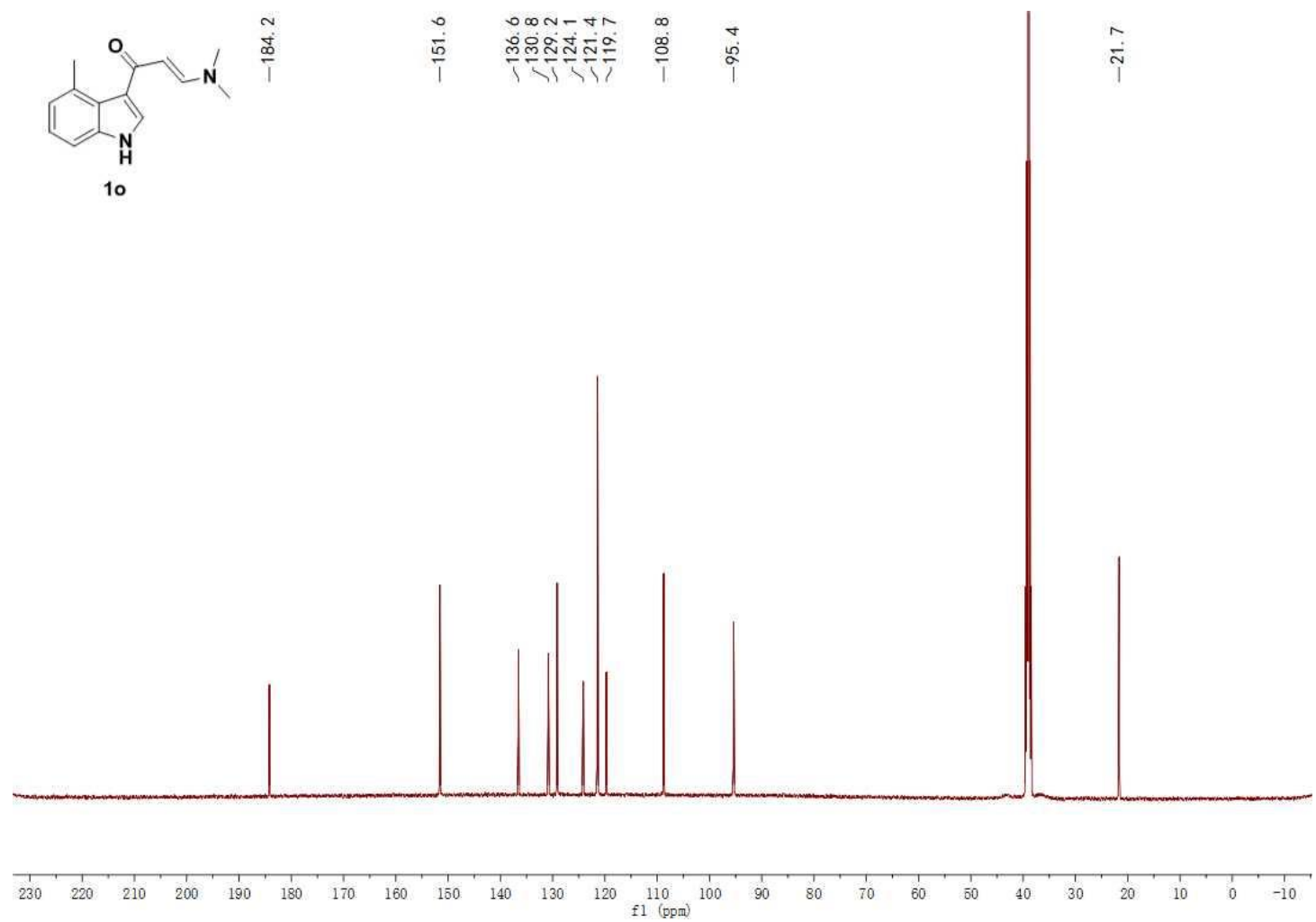
${ }^{1}$ H NMR (600 MHz, DMSO-d6)

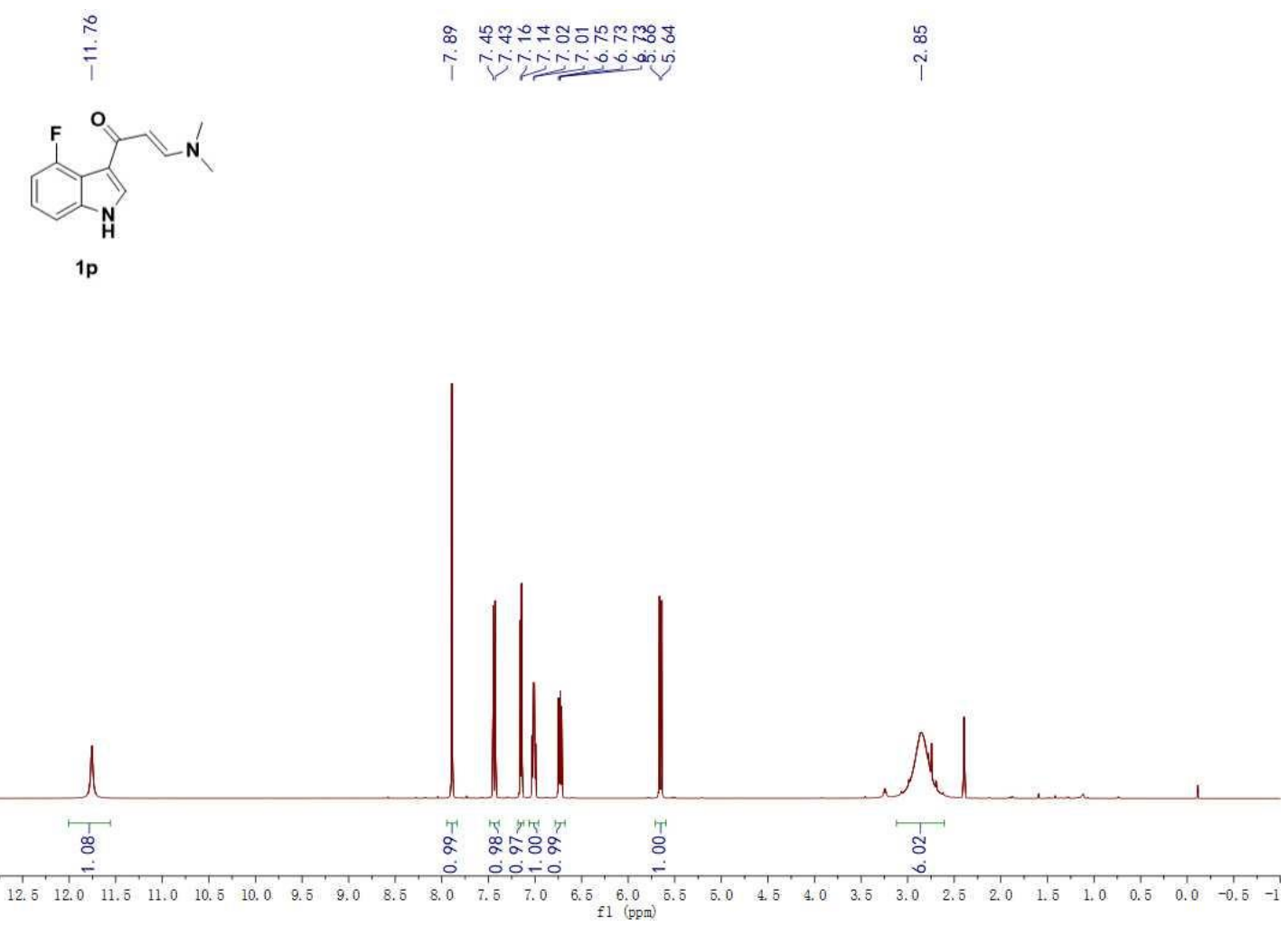

${ }^{13}$ C NMR (150 MHz, DMSO-d6)
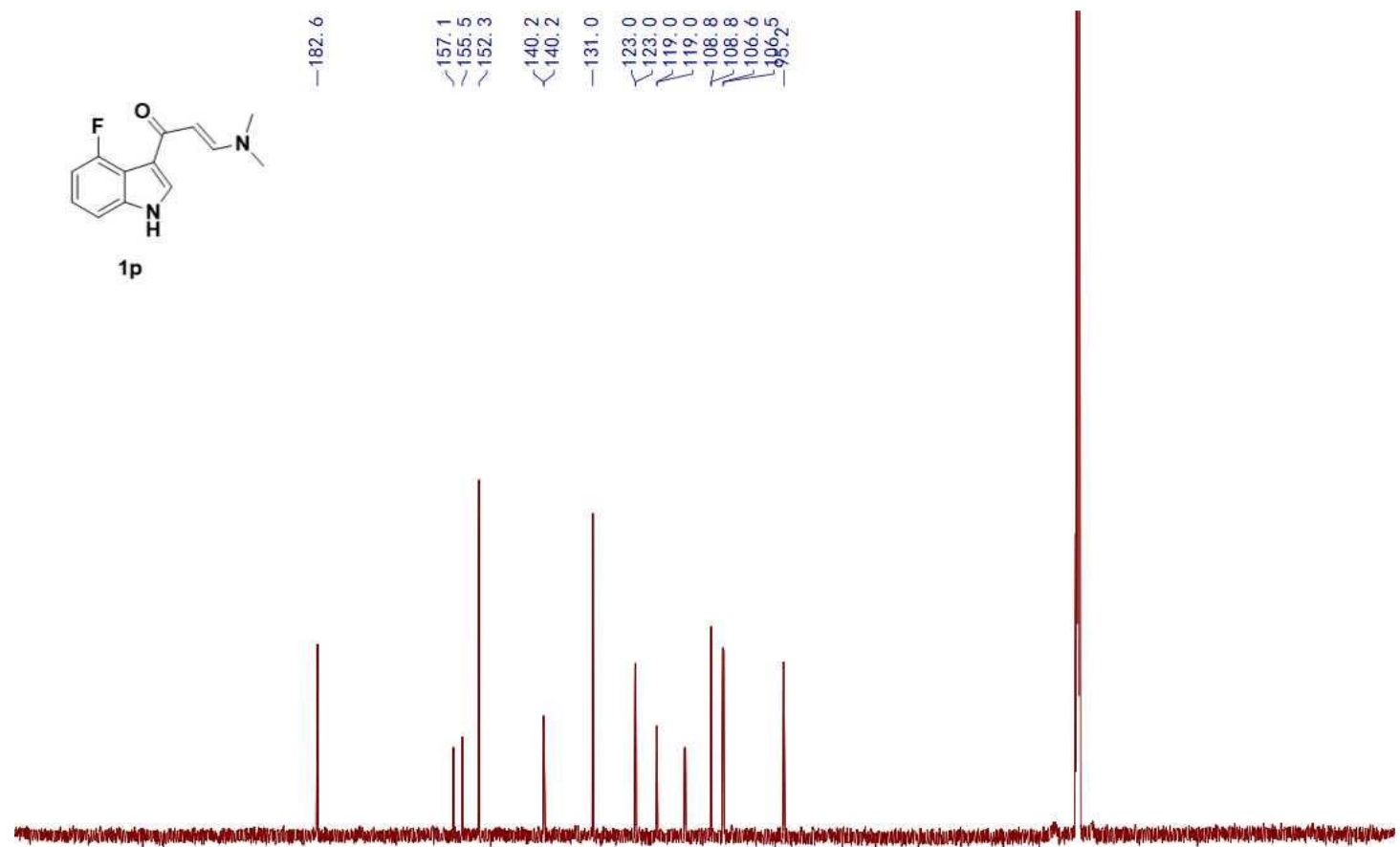

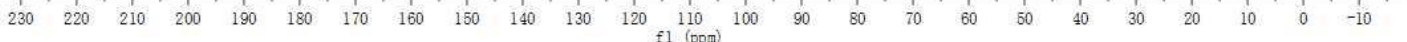


${ }^{1}$ H NMR (600 MHz, DMSO-d6)

$\stackrel{i}{\stackrel{1}{=}}$

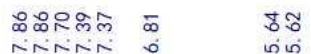

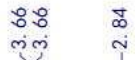

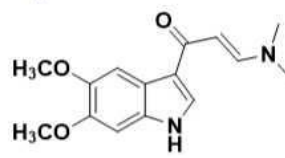

19

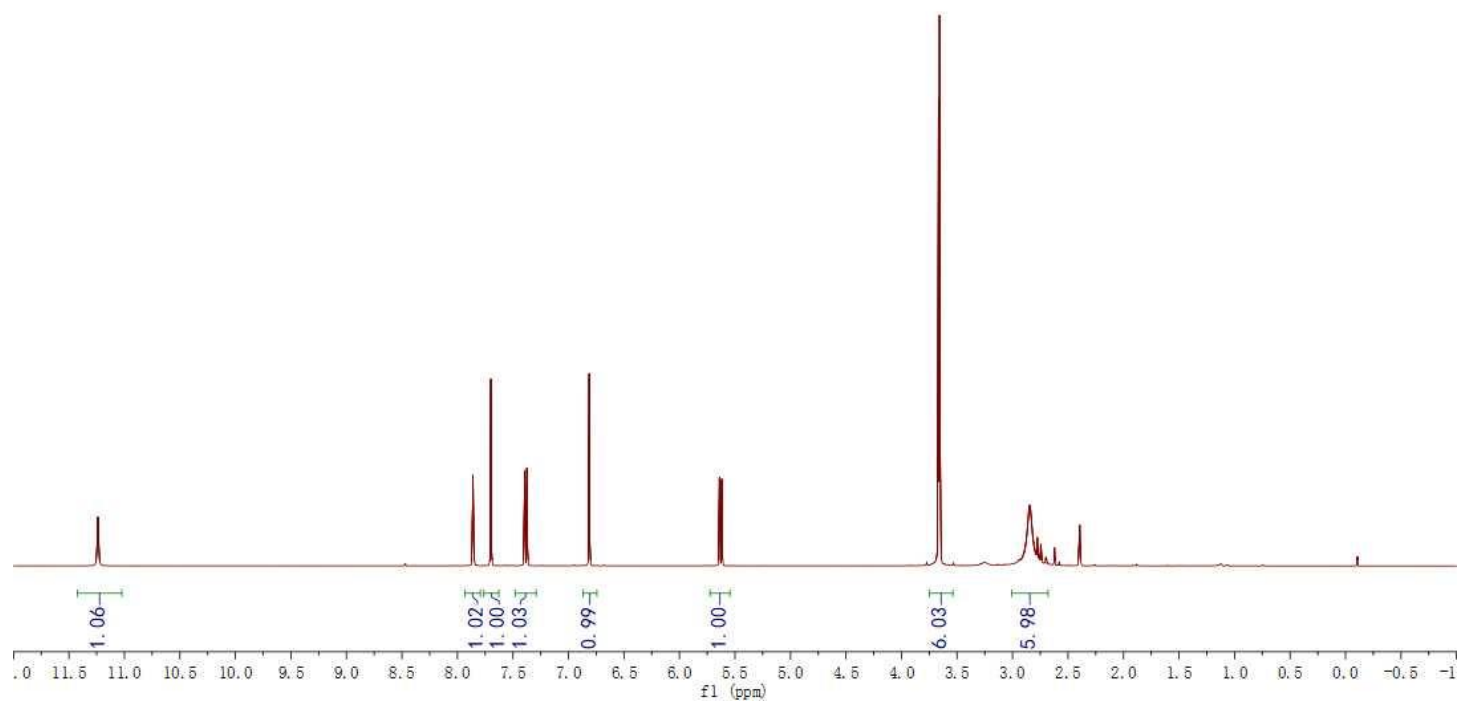

${ }^{13}$ C NMR (150 MHz, DMSO-d6)

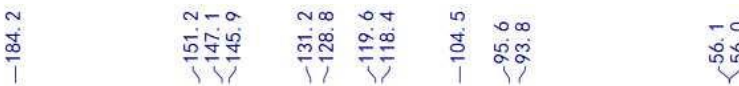

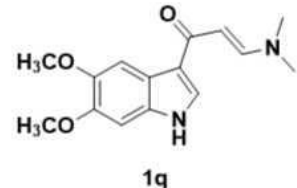

$1 q$

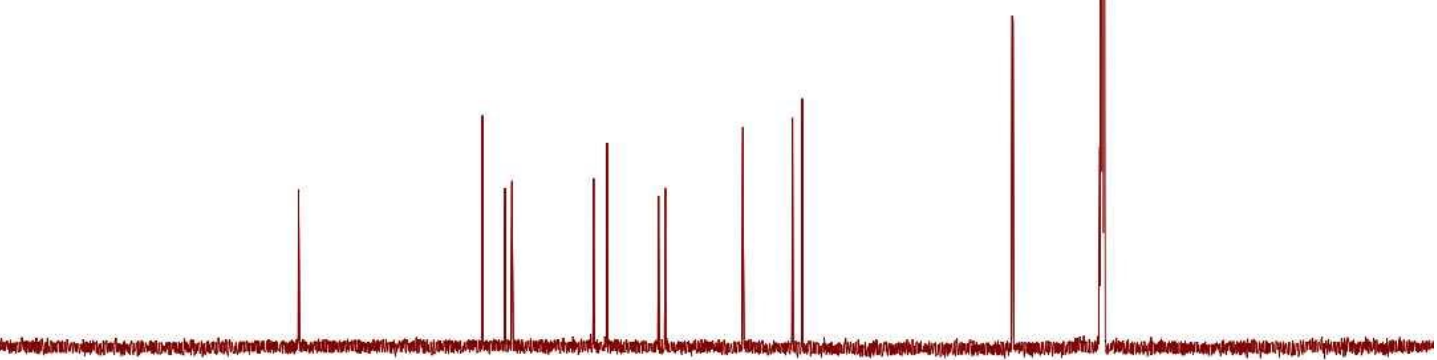

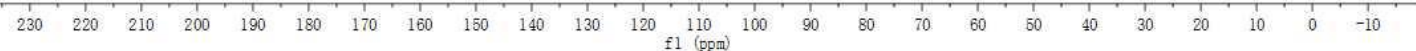


${ }^{1}$ H NMR (500 MHz, DMSO-d6)

\begin{tabular}{|c|c|c|}
\hline 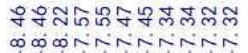 & $\begin{array}{l}\text { Ki: } \\
\text { nin }\end{array}$ & $\begin{array}{c}\infty \\
\infty \\
m\end{array}$ \\
\hline
\end{tabular}
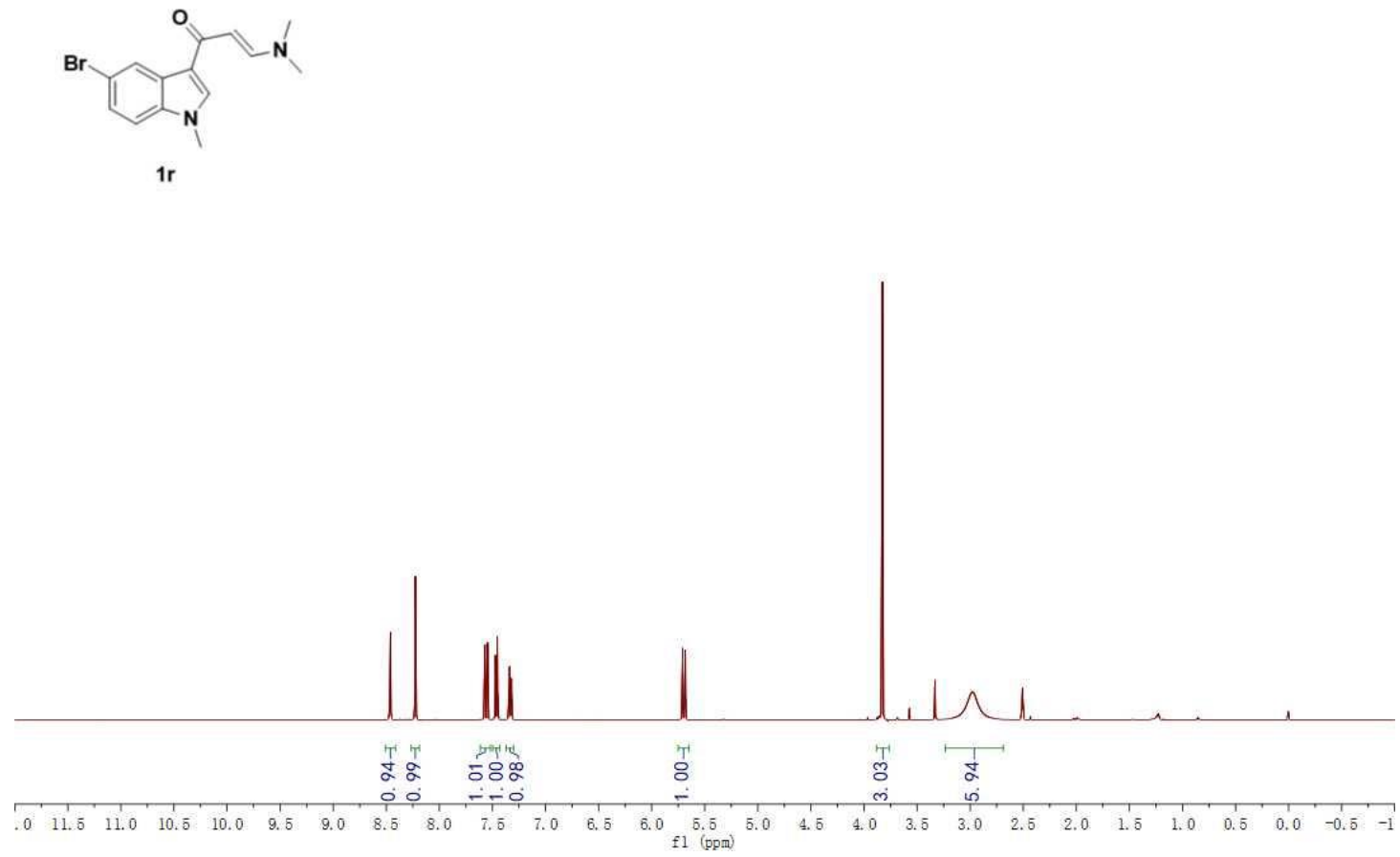

${ }^{13}$ C NMR (125 MHz, DMSO-d6)

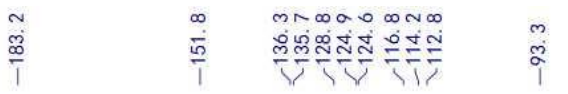

ल

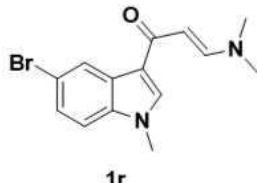

$1 \mathrm{r}$

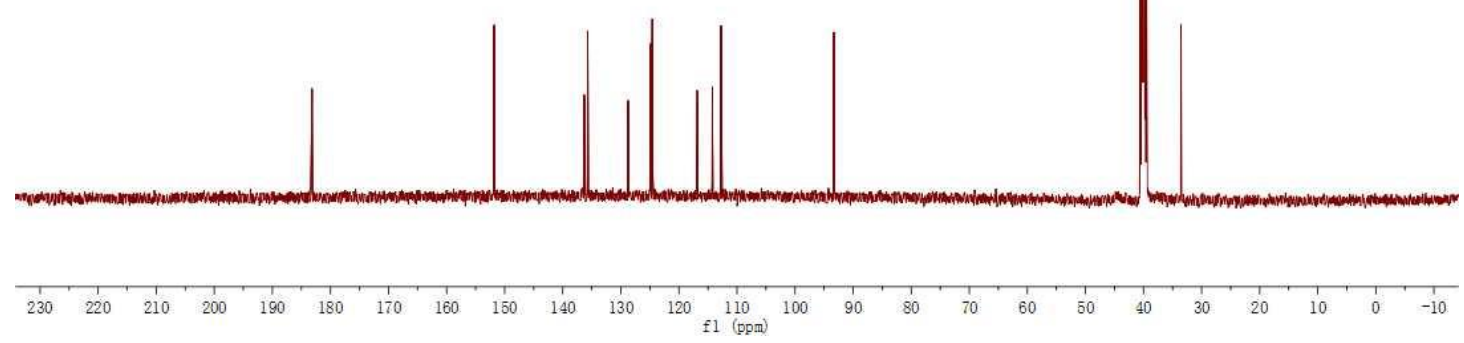


${ }^{1}$ H NMR (500 MHz, DMSO-d6)

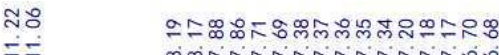

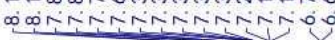

भํํㅇำ

ن⿺辶万

ळ్లిలి
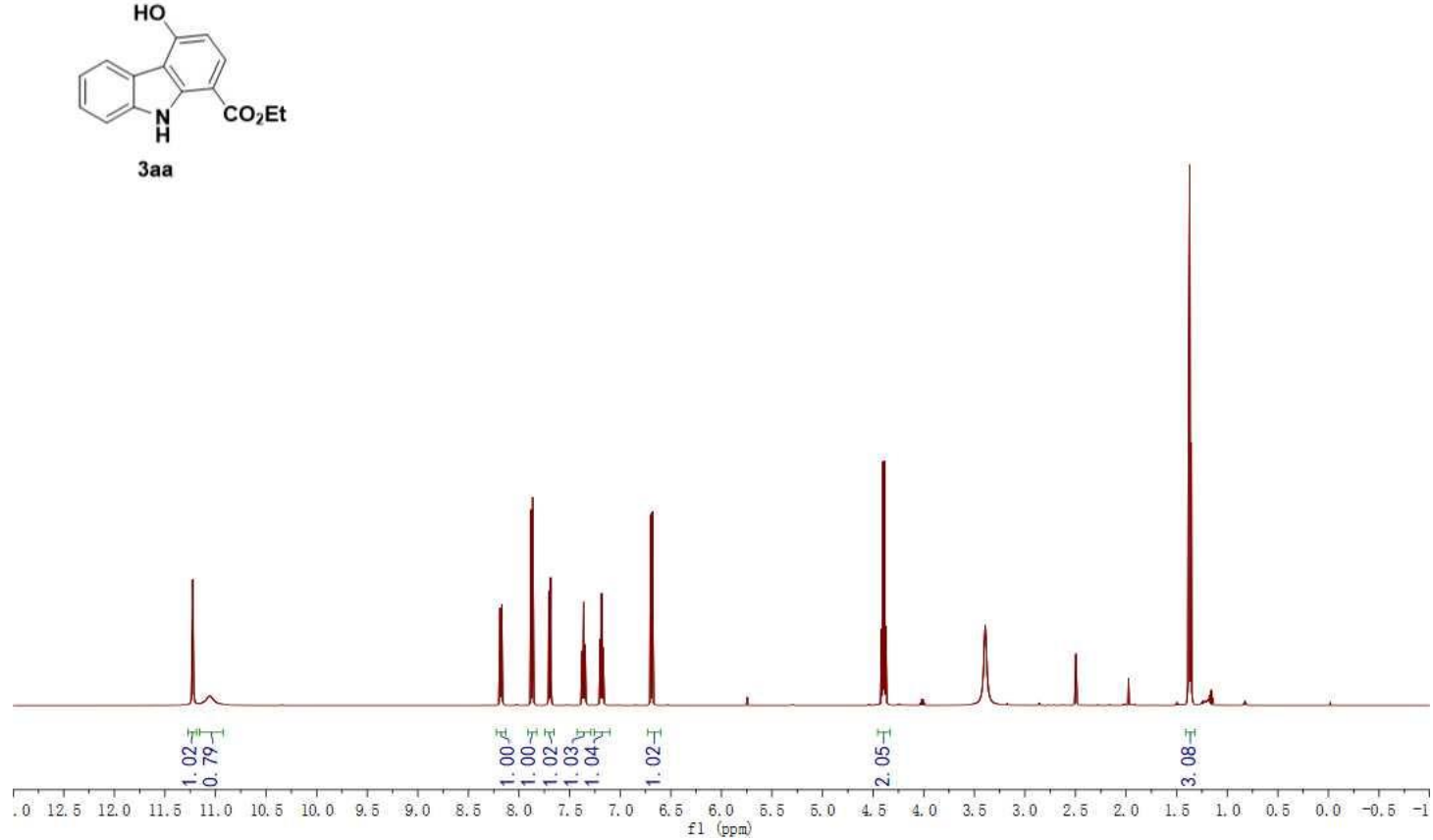

${ }^{13}$ C NMR (125 MHz, DMSO-d6)

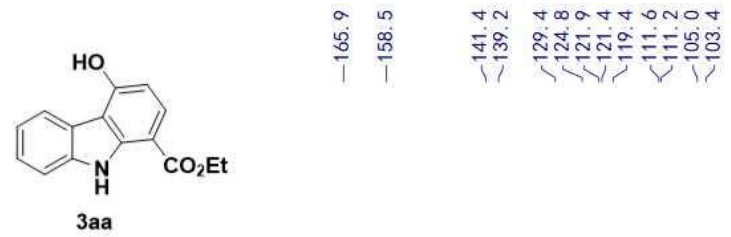

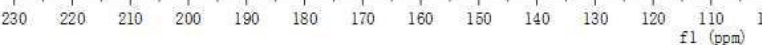


${ }^{1}$ H NMR (500 MHz, DMSO-d6)
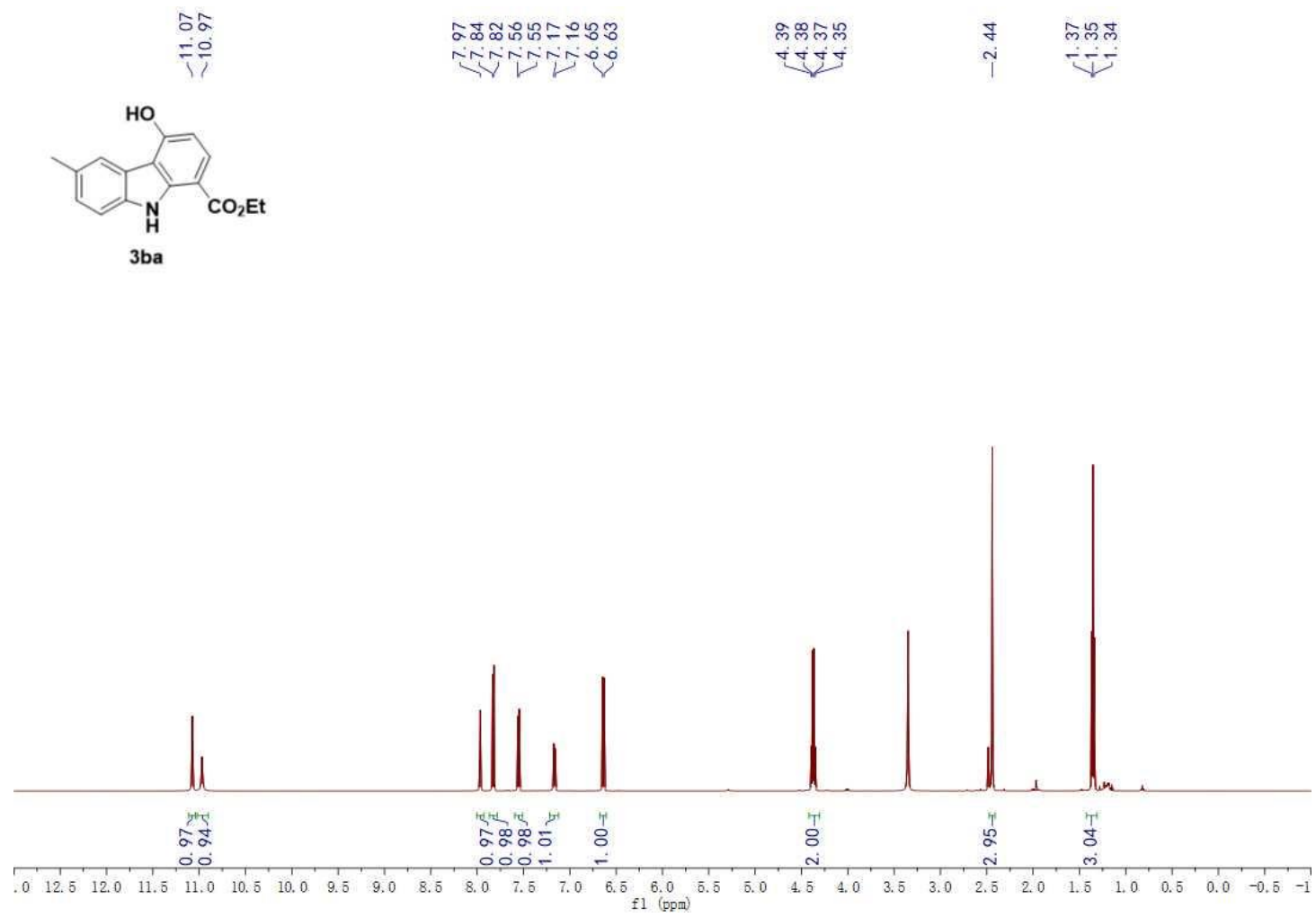

${ }^{13}$ C NMR (125 MHz, DMSO-d6)

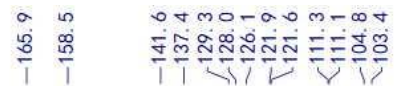

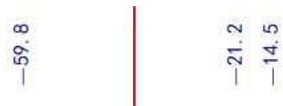

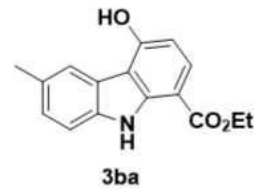

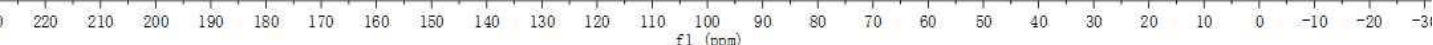


${ }^{1}$ H NMR (600 MHz, DMSO-d6)

है
$\frac{1}{1}$
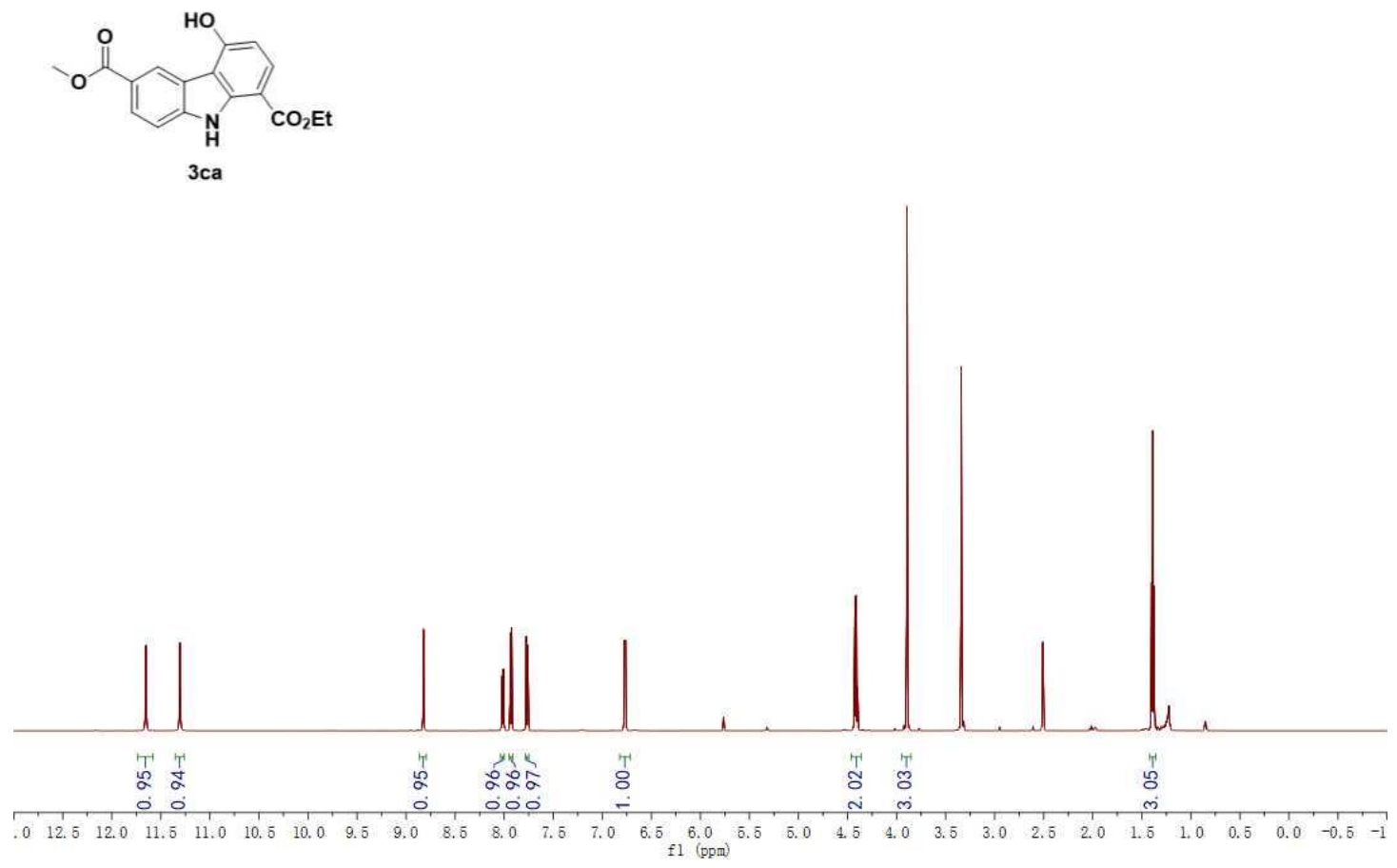

${ }^{13}$ C NMR (150 MHz, DMSO-d6)
भู่ะ์

迷向 
${ }^{1}$ H NMR (500 MHz, DMSO-d6)

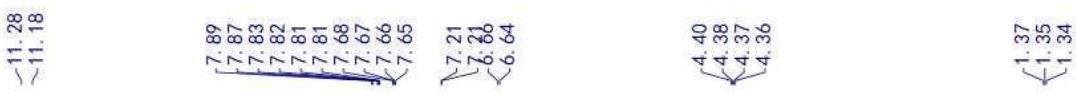
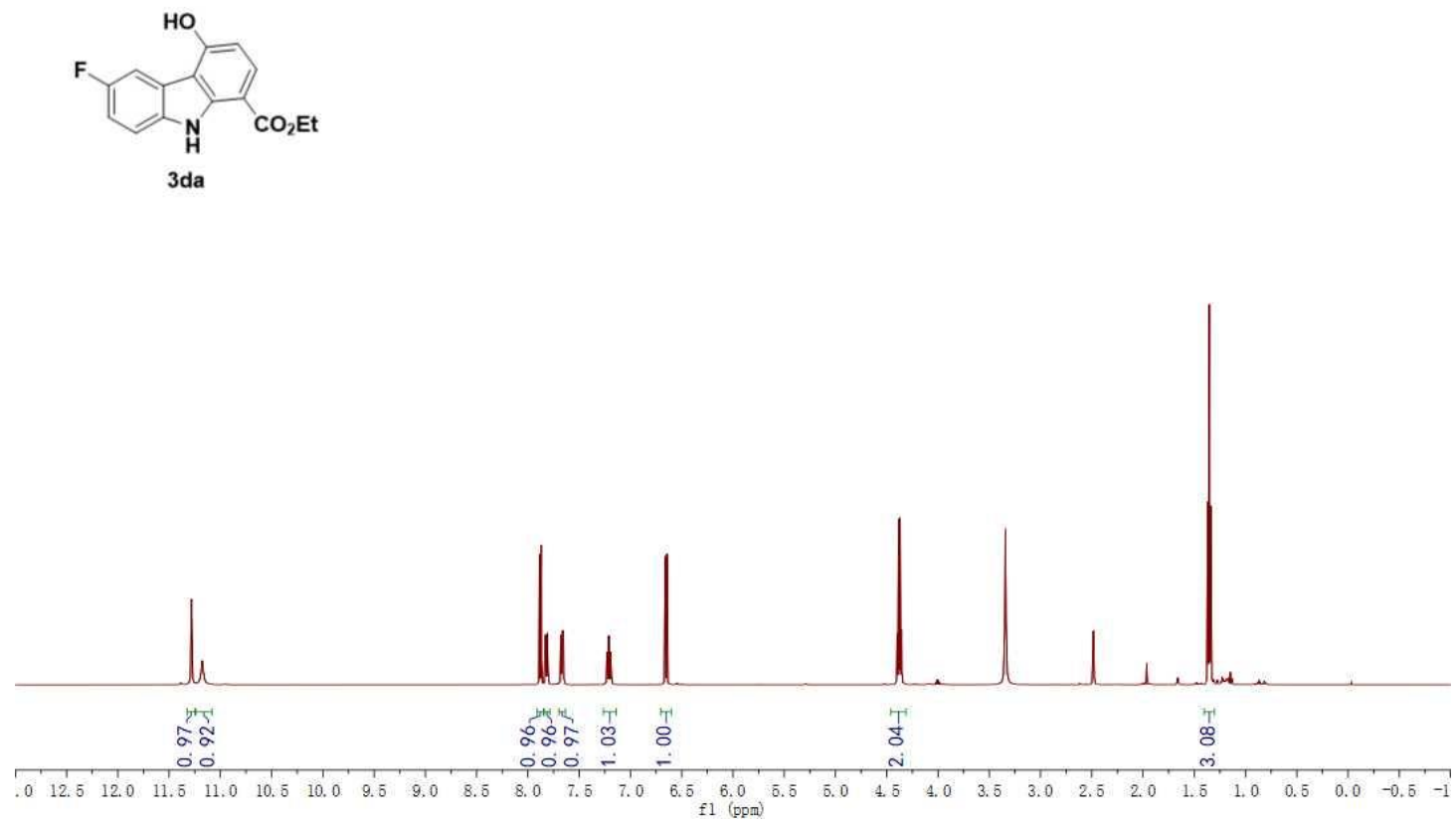

${ }^{13}$ C NMR (125 MHz, DMSO-d6)

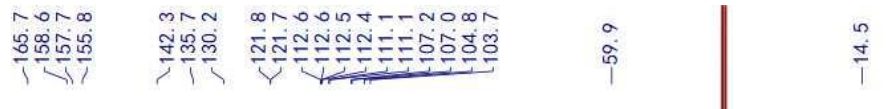
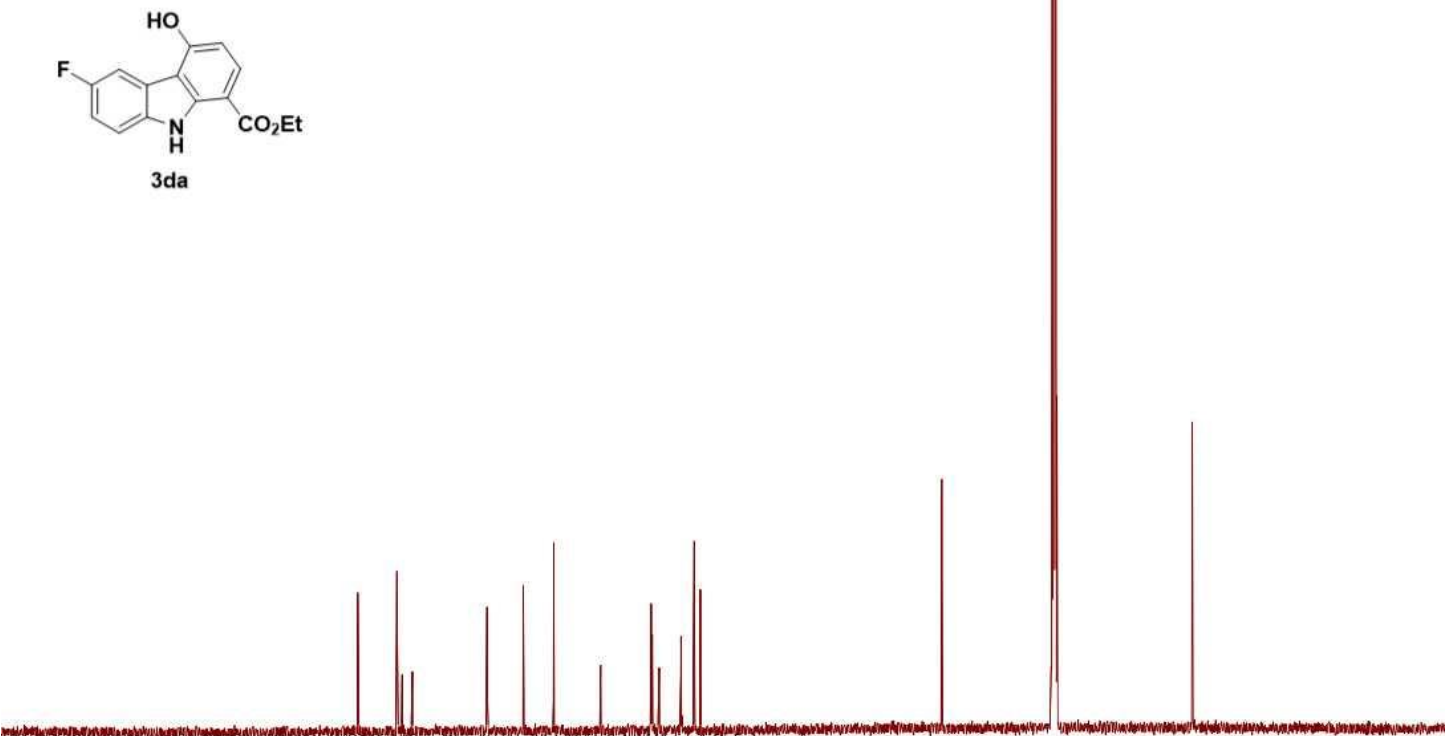

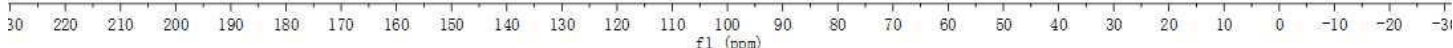


${ }^{1}$ H NMR (500 MHz, DMSO-d6)

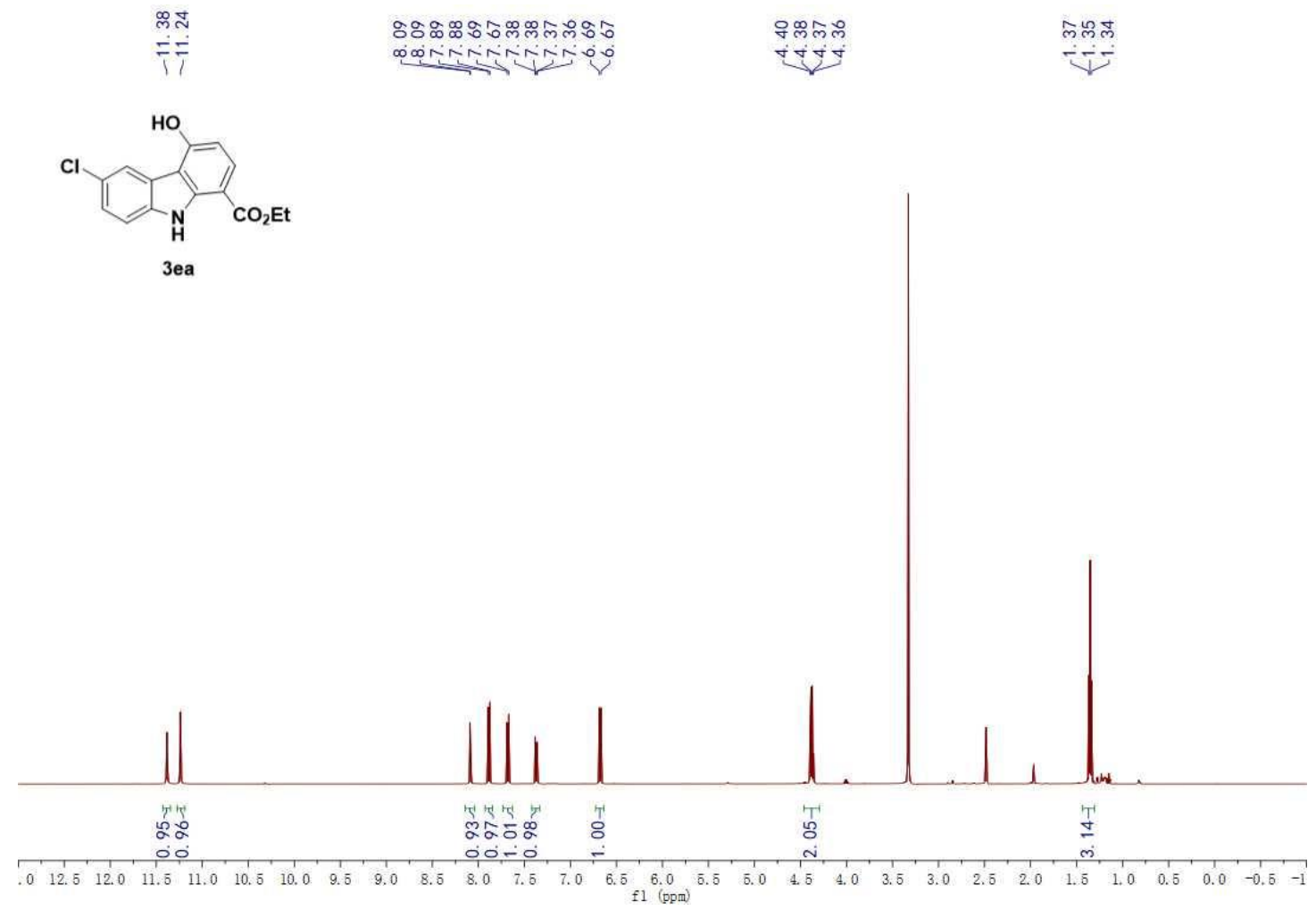

${ }^{13}$ C NMR (125 MHz, DMSO-d6)
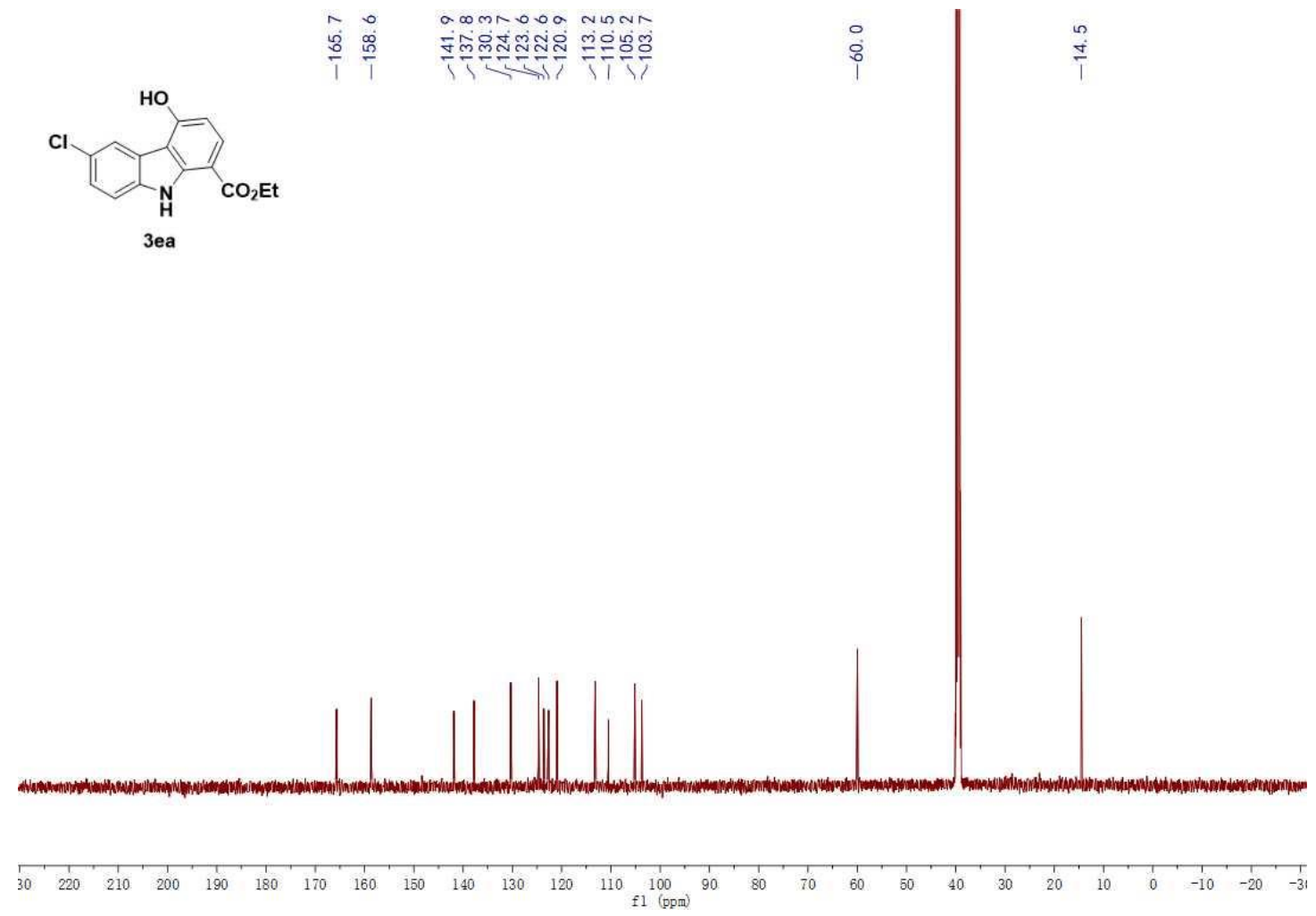
${ }^{1}$ H NMR (500 MHz, DMSO-d6)

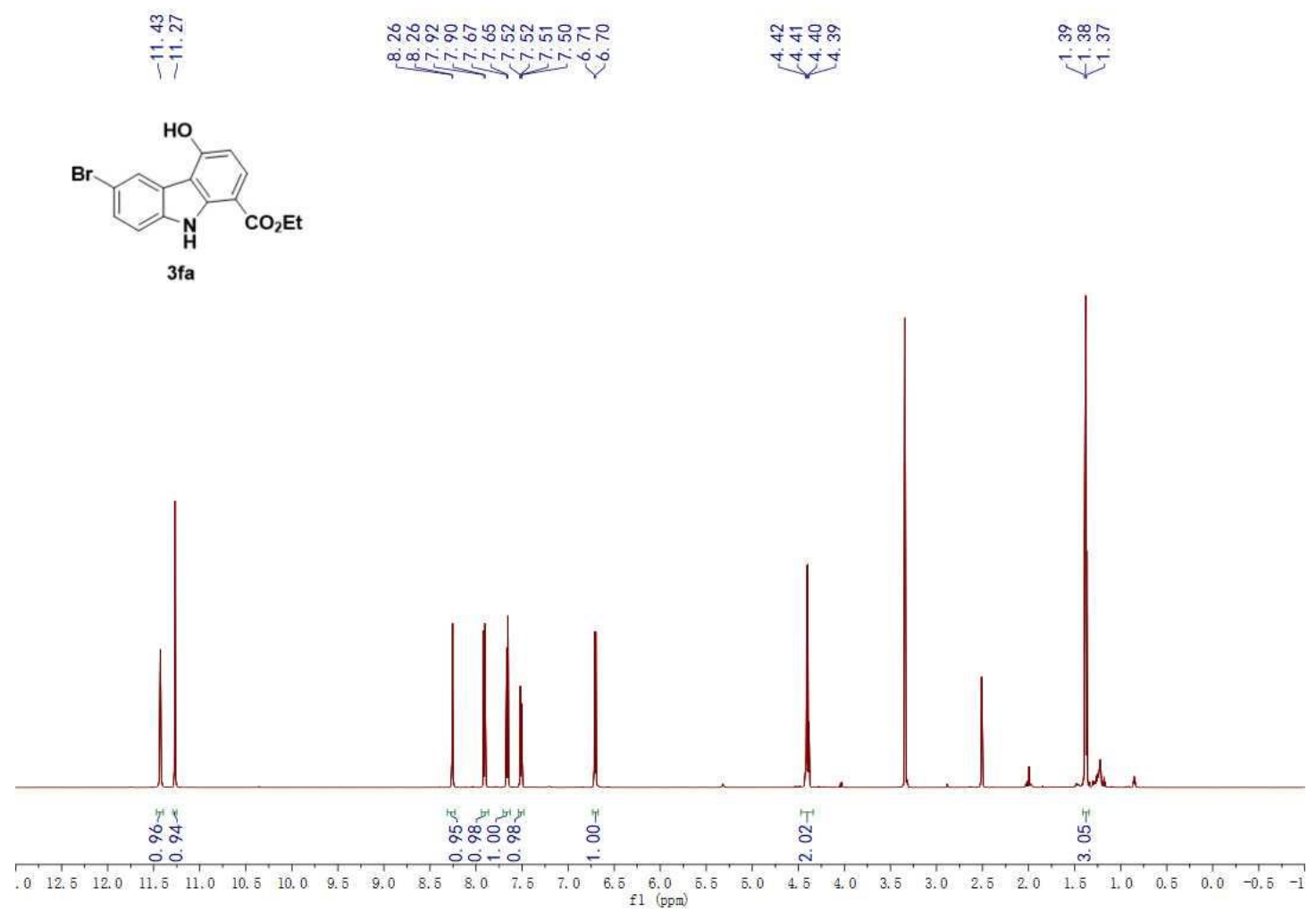

${ }^{13}$ C NMR (125 MHz, DMSO-d6)

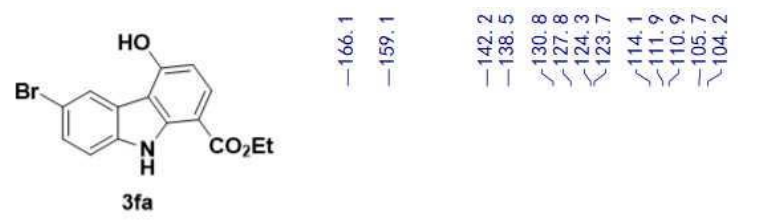

$$
\text { | }
$$

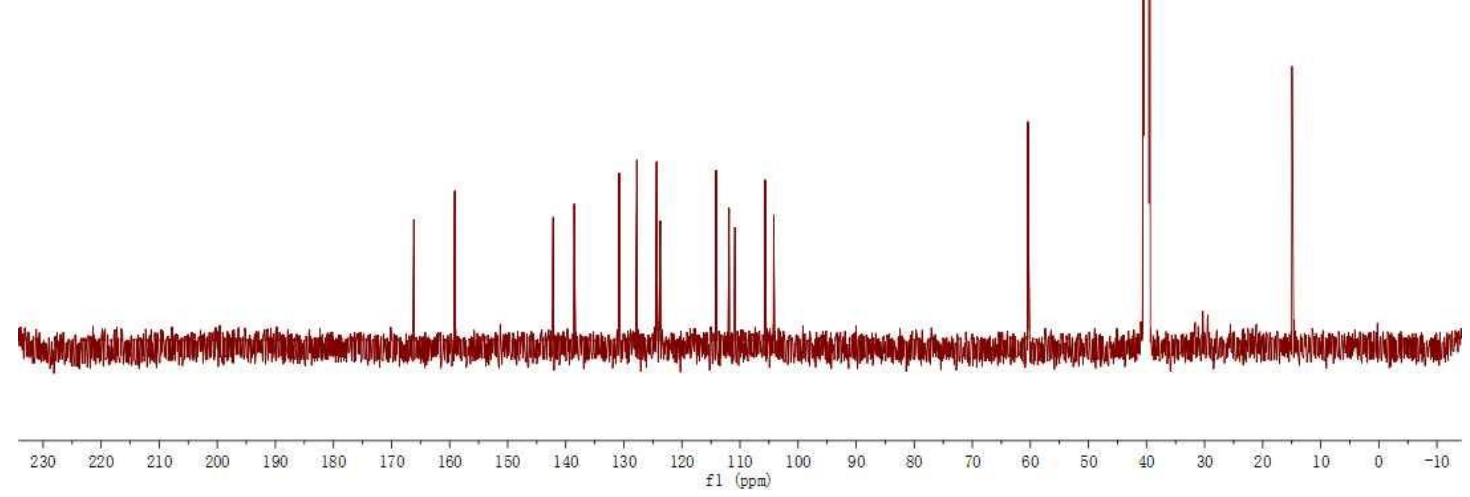


${ }^{1}$ H NMR (500 MHz, DMSO-d6)

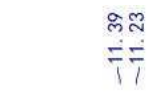

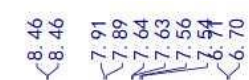

₹애

过茫

ळळ్
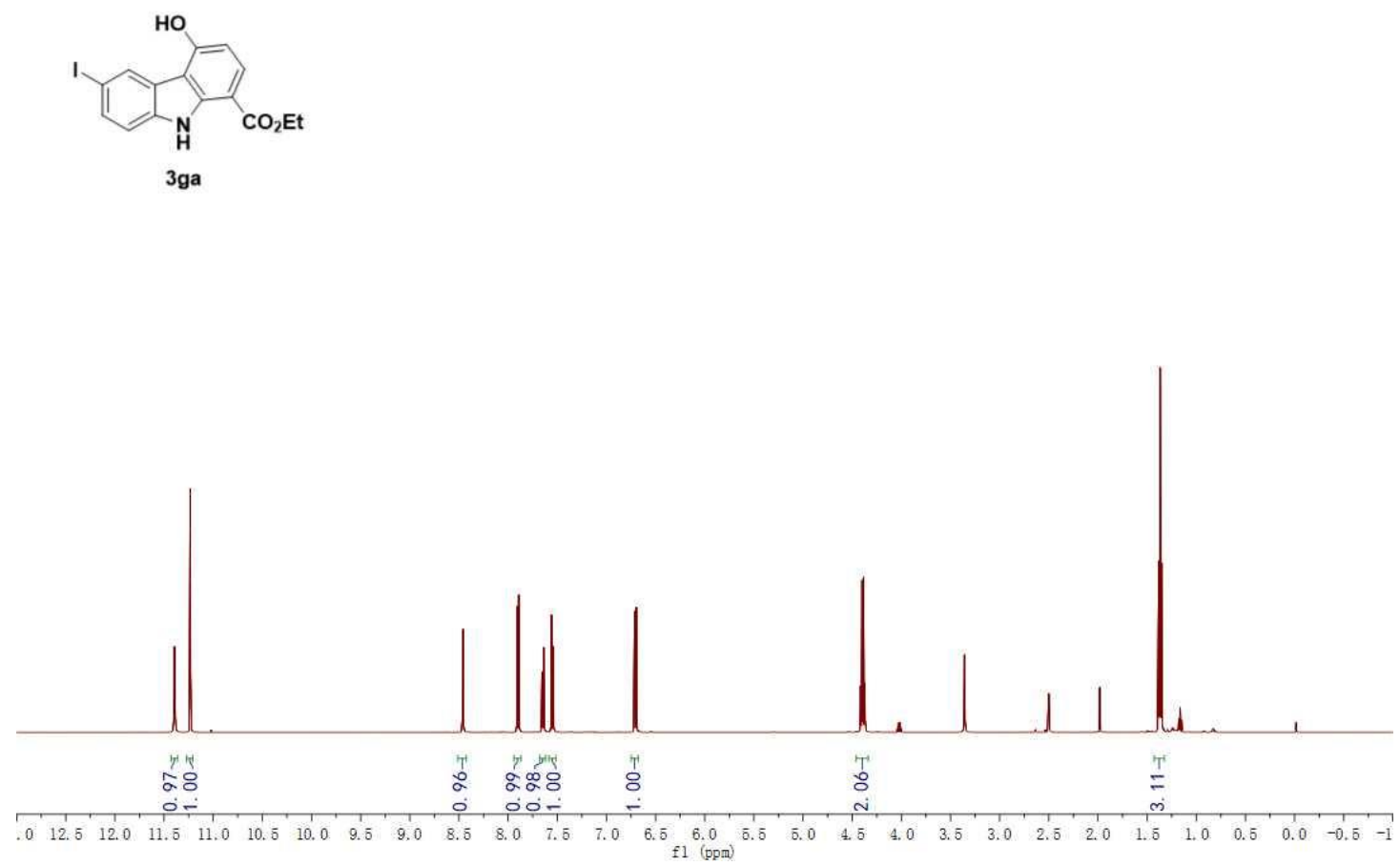

${ }^{13}$ C NMR (125 MHz, DMSO-d6)
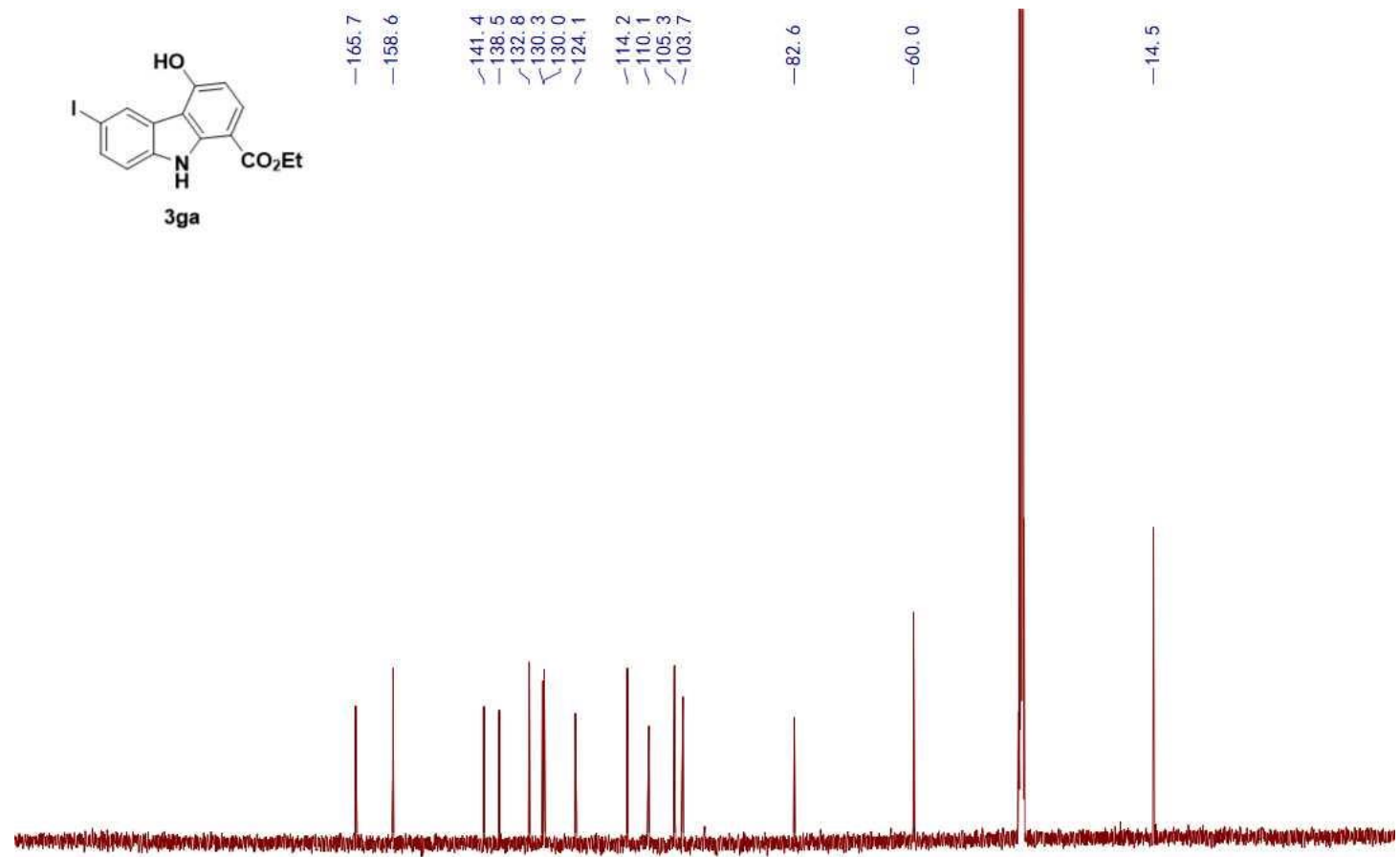

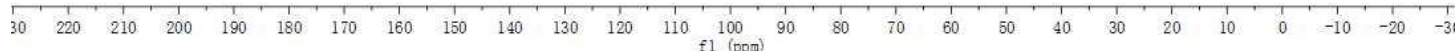


${ }^{1}$ H NMR (500 MHz, DMSO-d6)
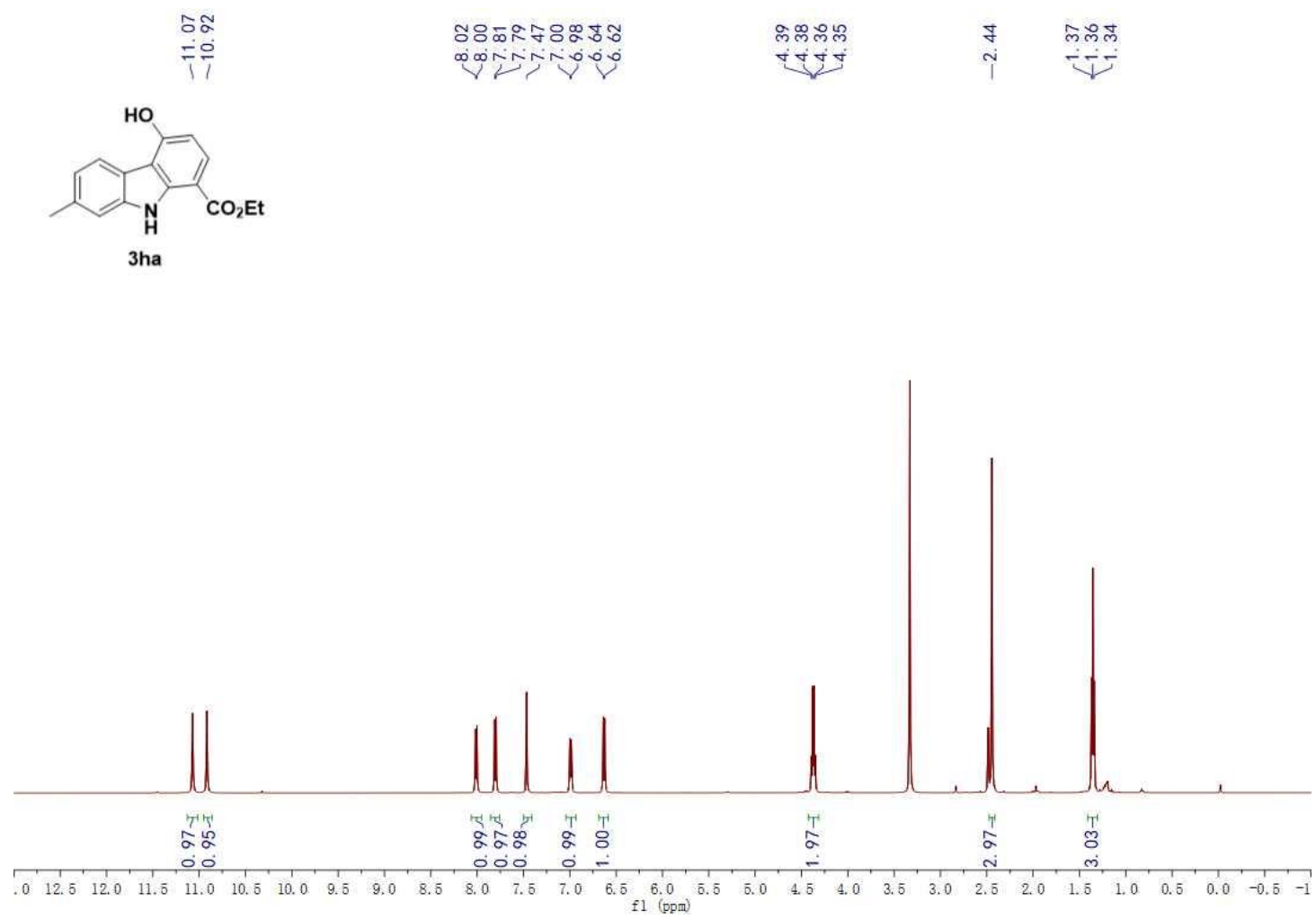

${ }^{13}$ C NMR (125 MHz, DMSO-d6)
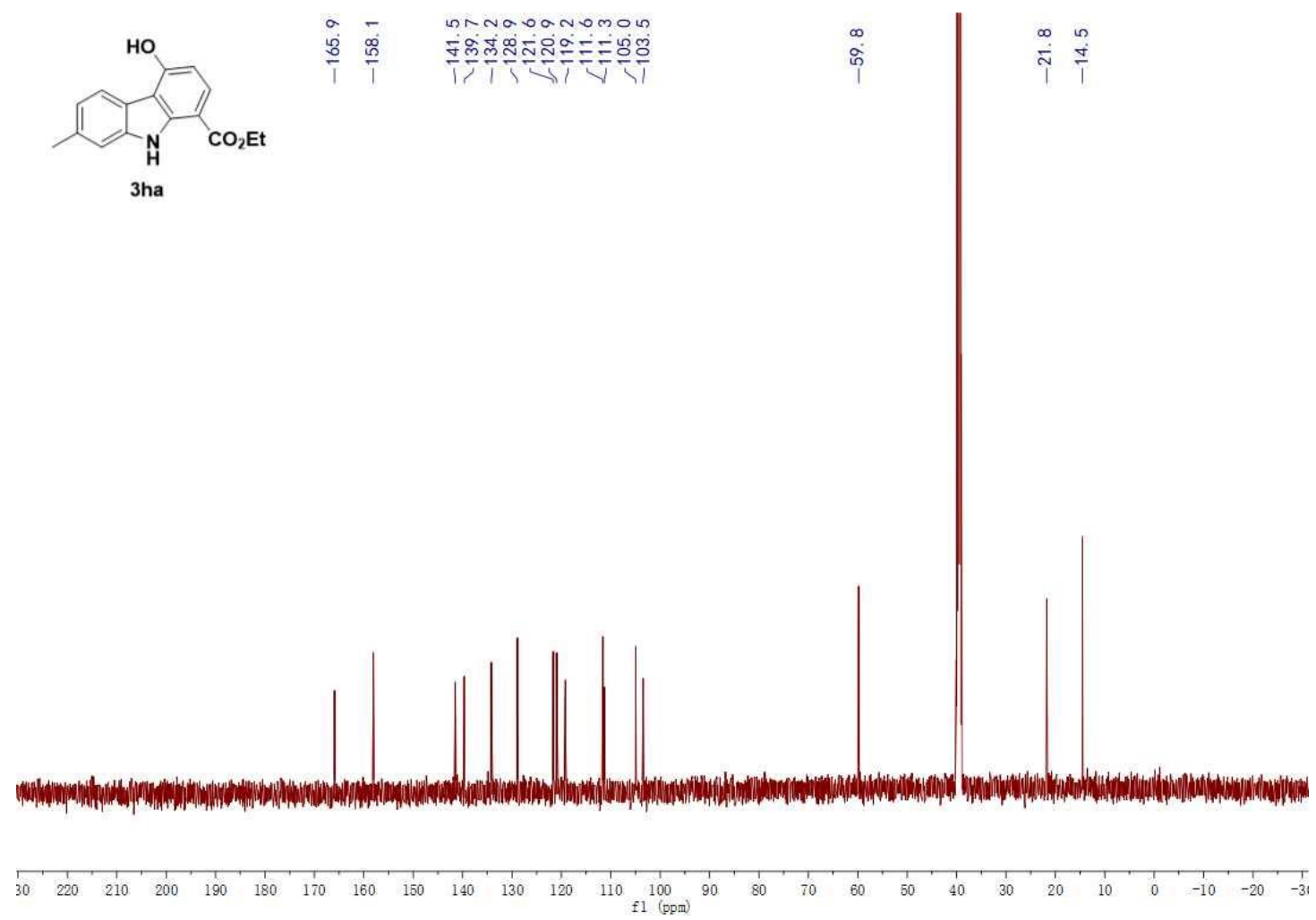
${ }^{1}$ H NMR (500 MHz, DMSO-d6)

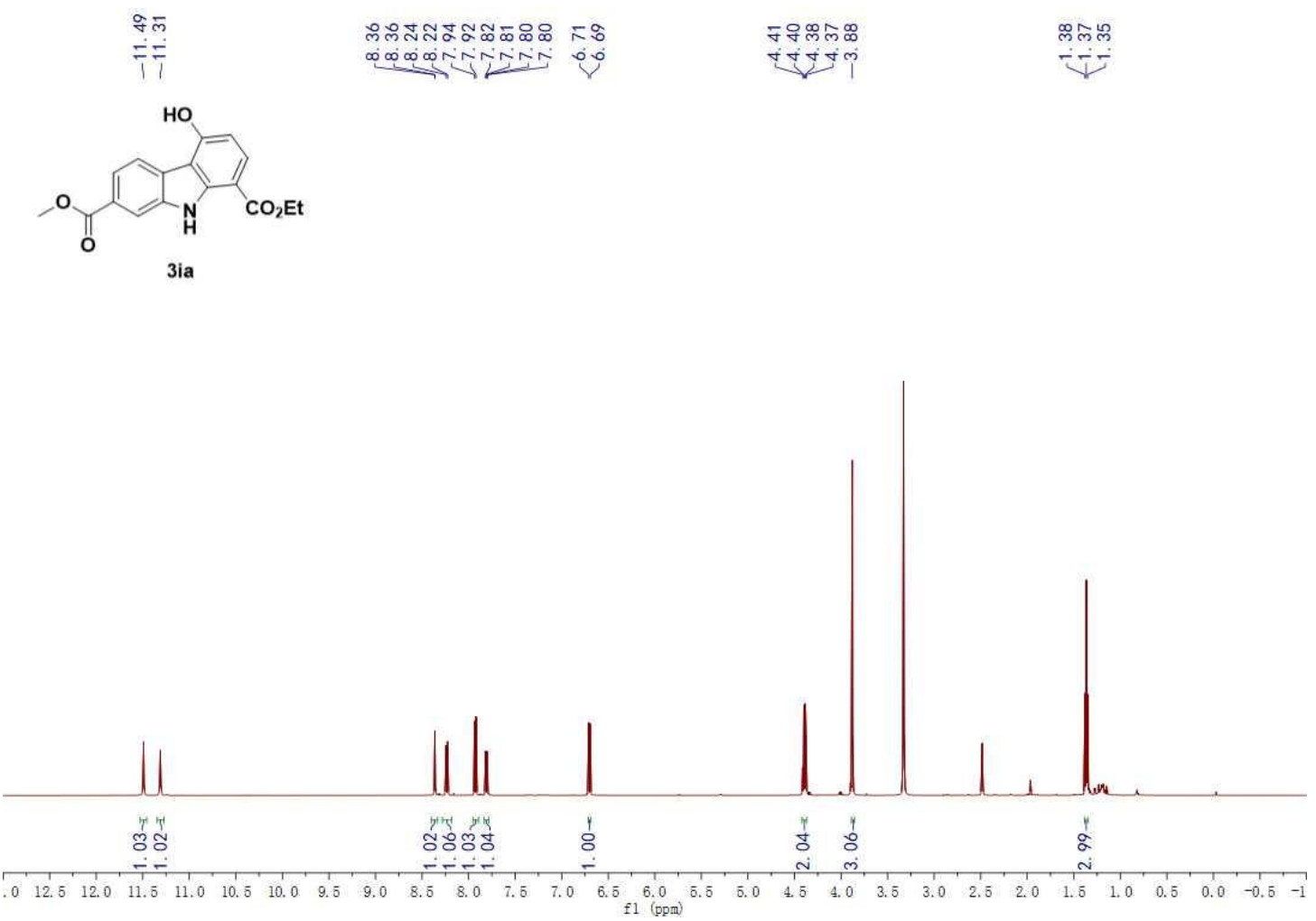

${ }^{13}$ C NMR (125 MHz, DMSO-d6)

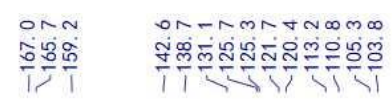

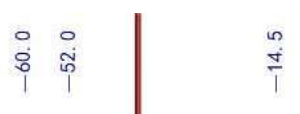
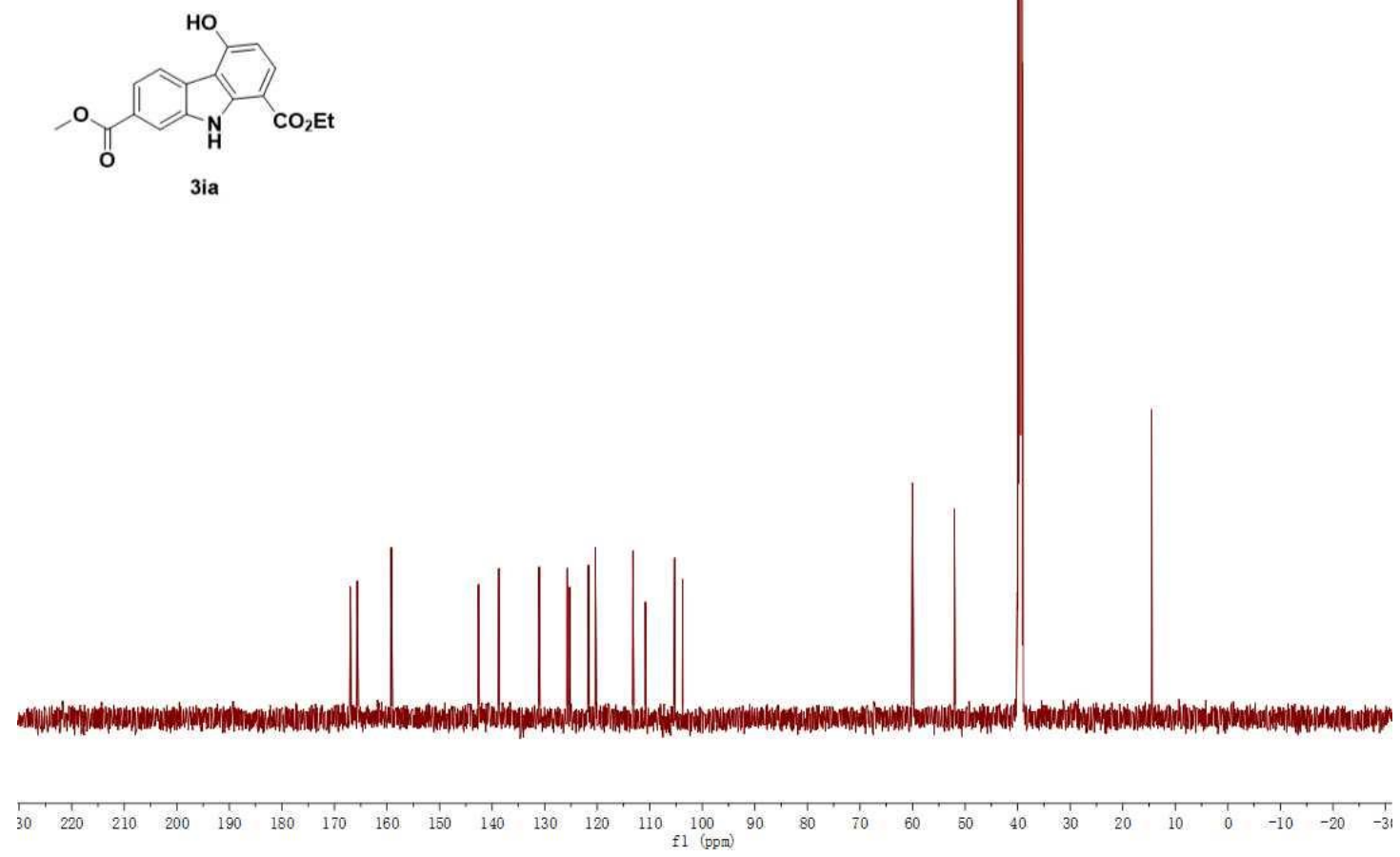
${ }^{1}$ H NMR (500 MHz, DMSO-d6)

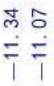

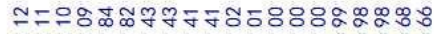

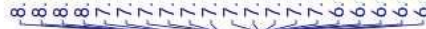

이요요

$\dot{i+i z}$

Hо

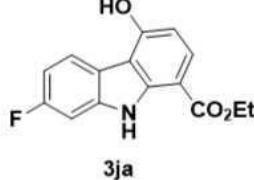

3ja

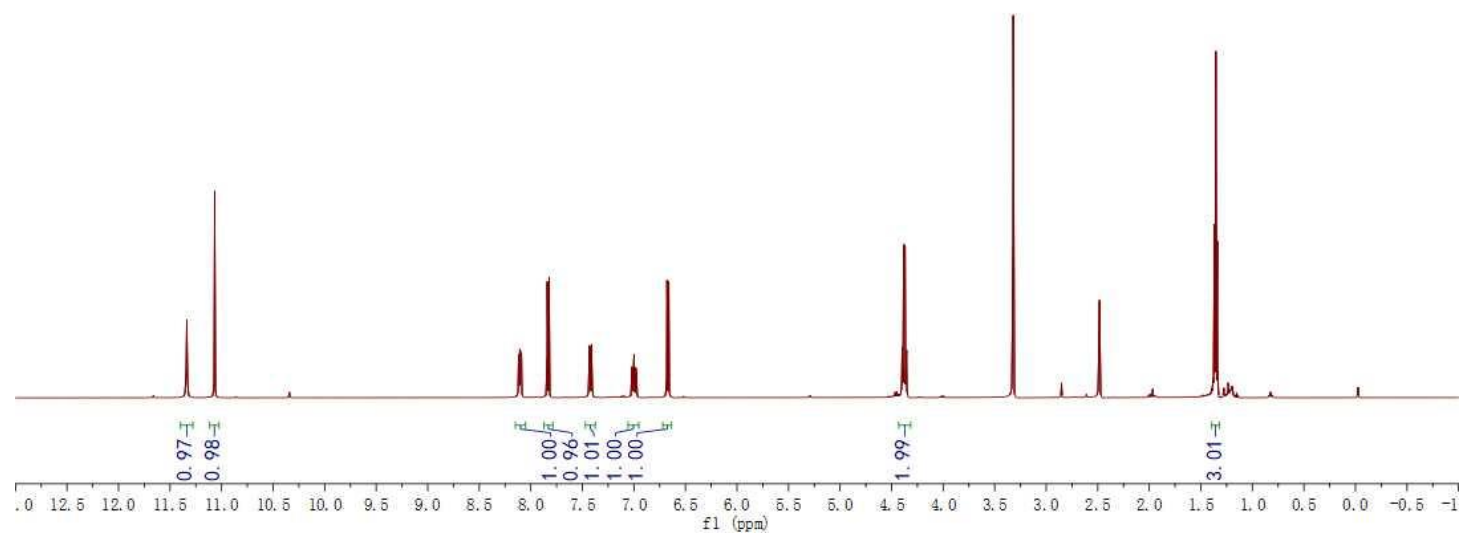

${ }^{13}$ C NMR (125 MHz, DMSO-d6)

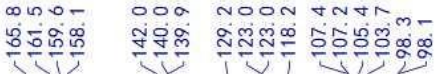

$\stackrel{1}{\circ} \quad \stackrel{0}{i}$
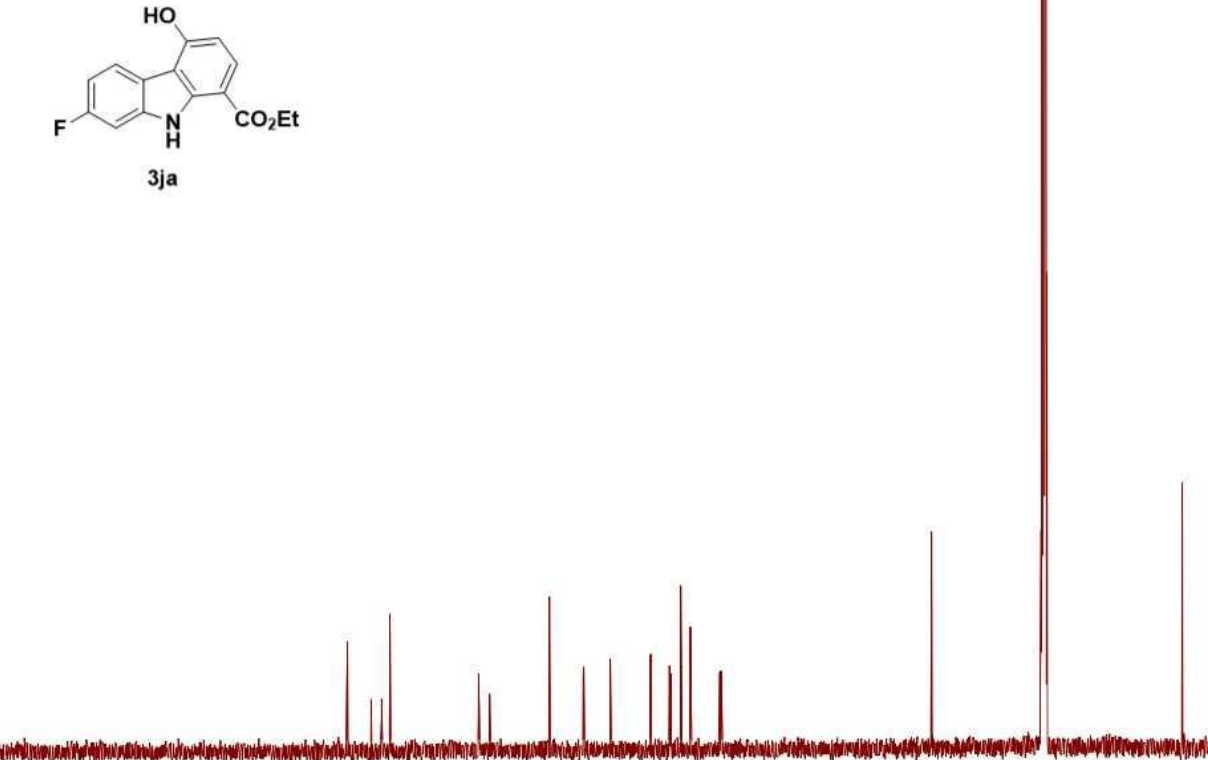

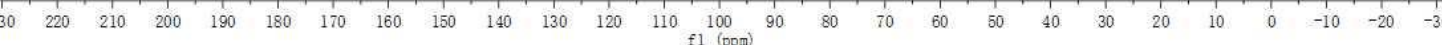


${ }^{1}$ H NMR (500 MHz, DMSO-d6)
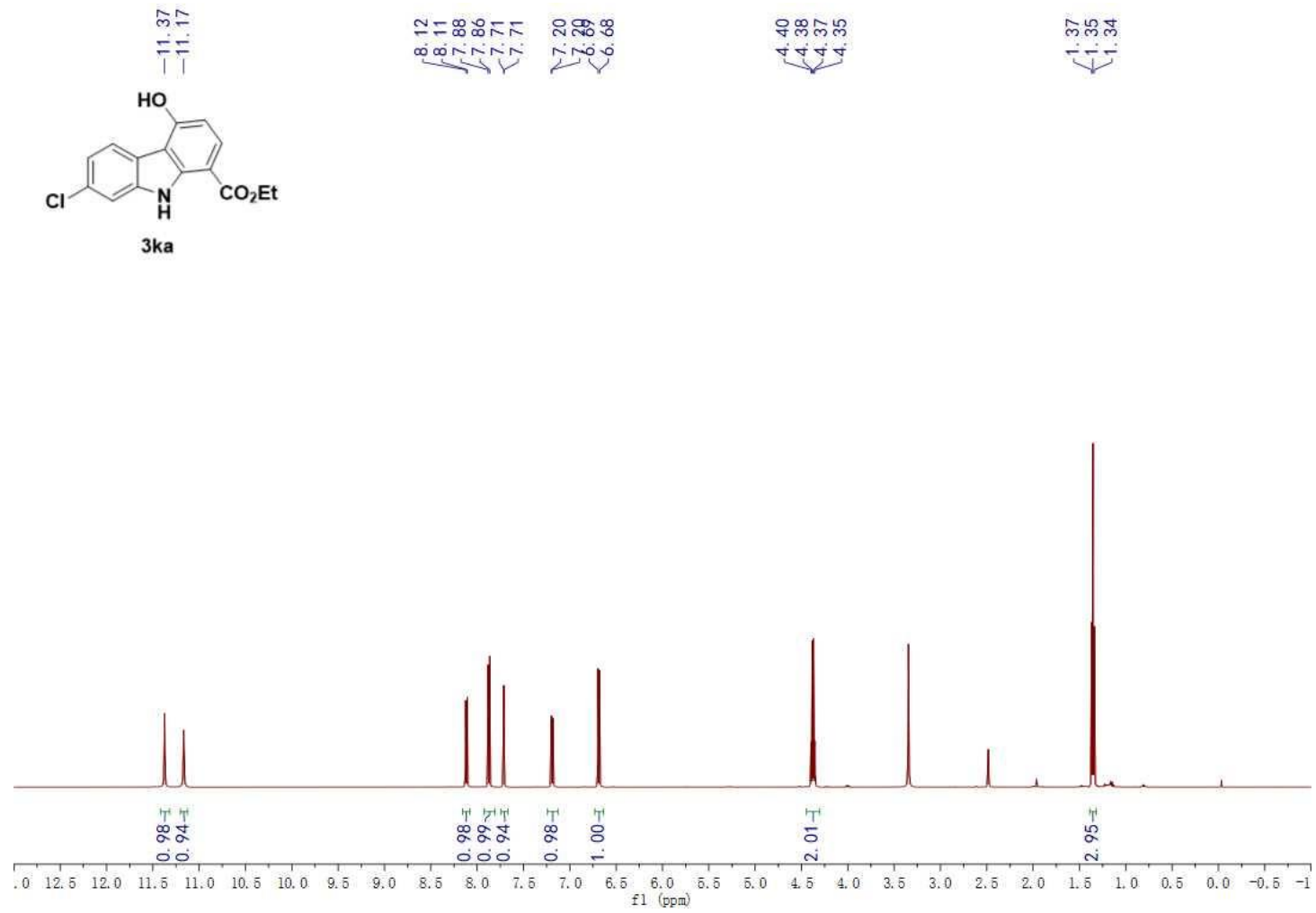

${ }^{13}$ C NMR (125 MHz, DMSO-d6)
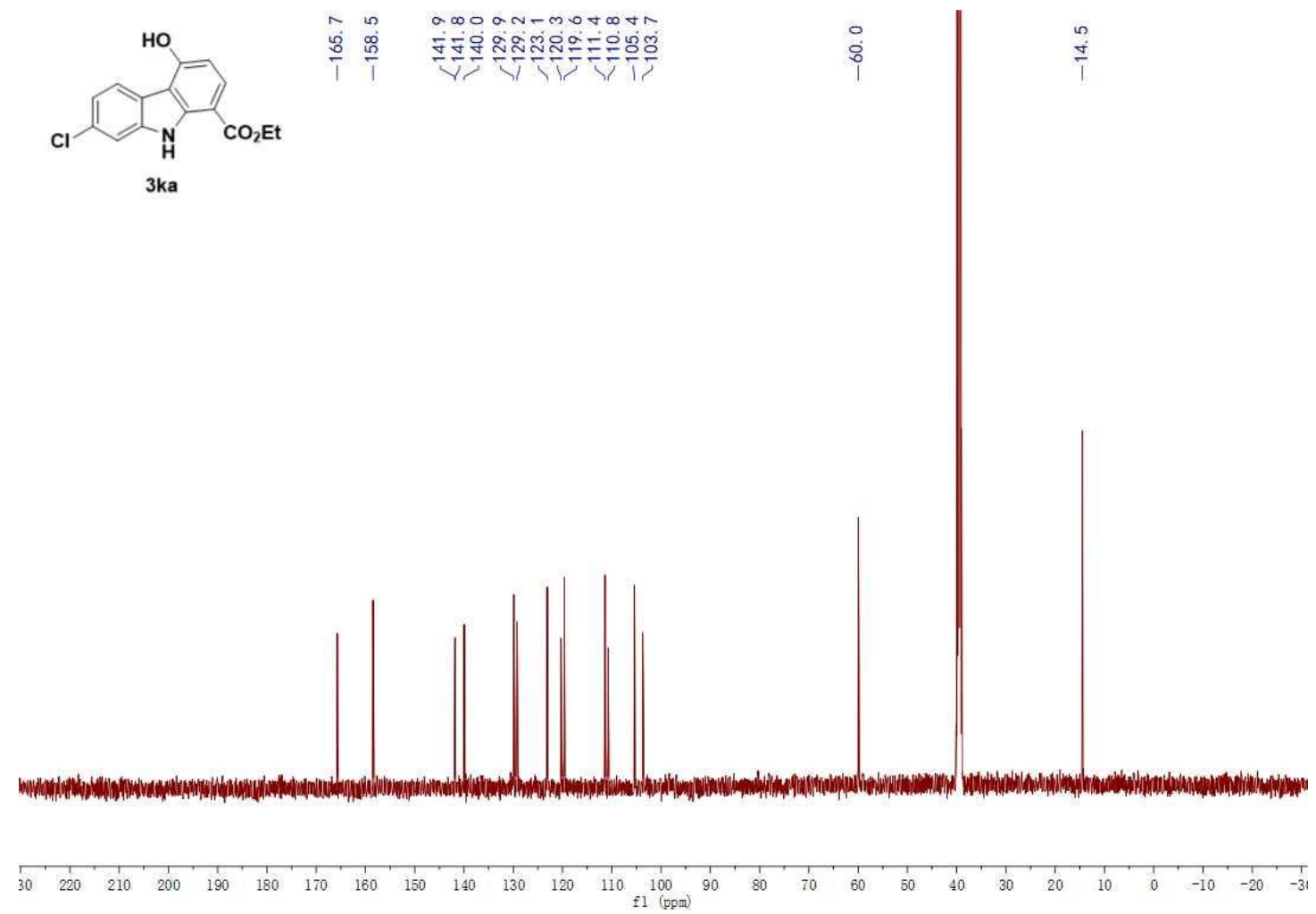
${ }^{1}$ H NMR (500 MHz, DMSO-d6)

$\stackrel{m}{=} \quad \stackrel{a}{i} \quad \stackrel{0}{i}$

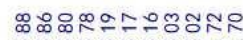

ninivinivio

ఫల్లోల్లం

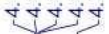

कूलेल

-
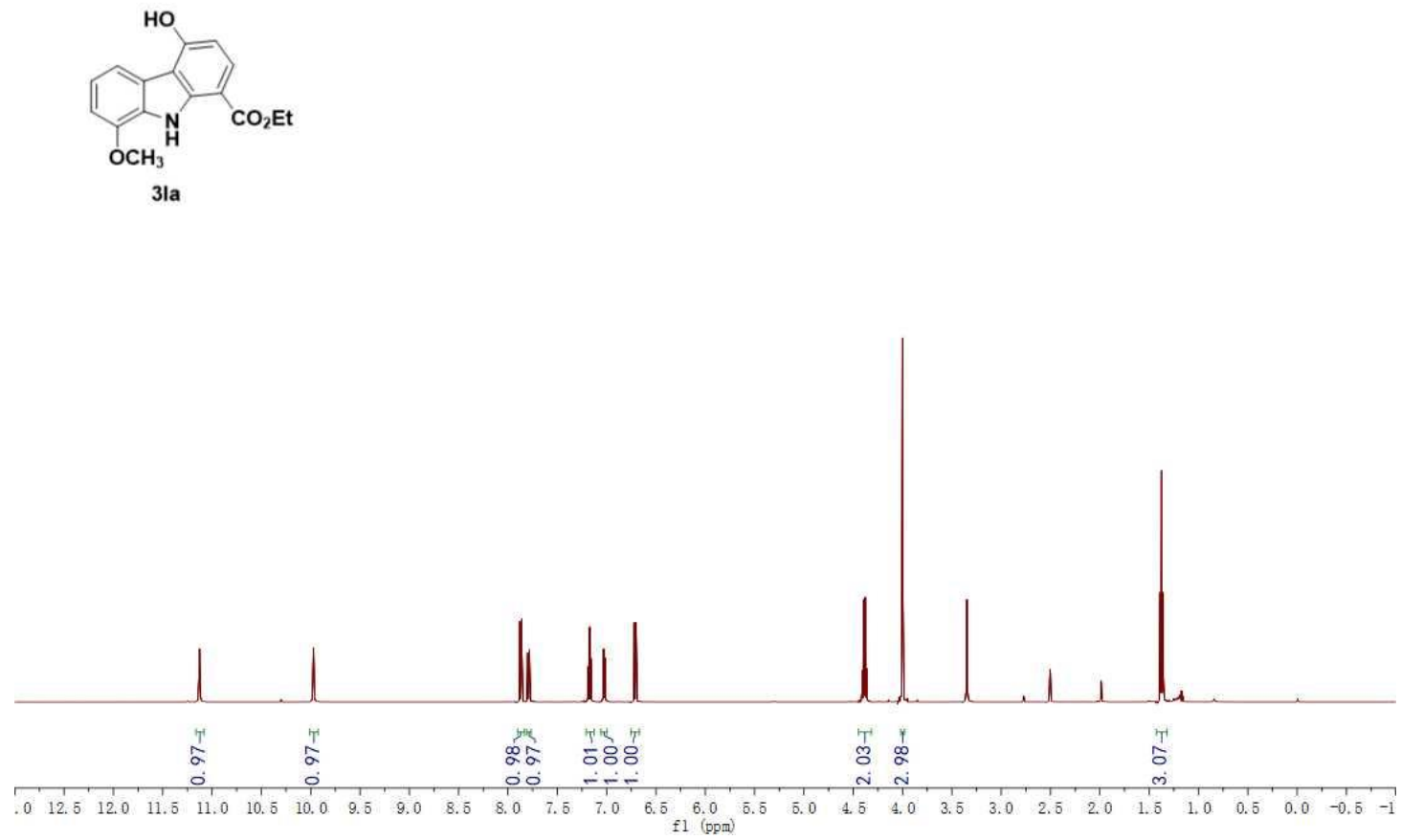

${ }^{13}$ C NMR (125 MHz, DMSO-d6)

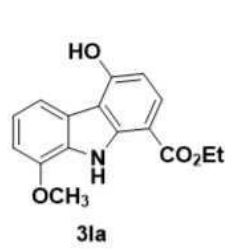

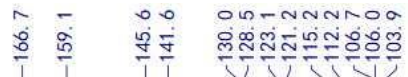

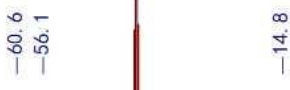

$31 \mathrm{a}$

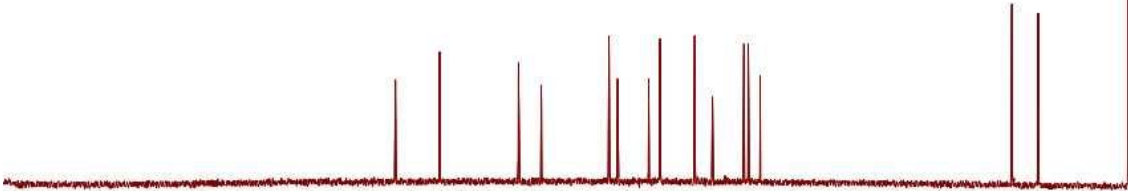

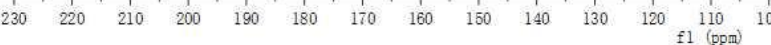


${ }^{1}$ H NMR (600 MHz, DMSO-d6)

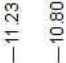

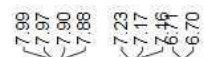

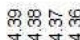

过文

Hо

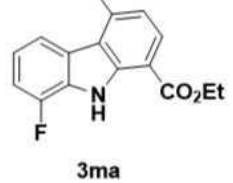

$3 \mathrm{ma}$

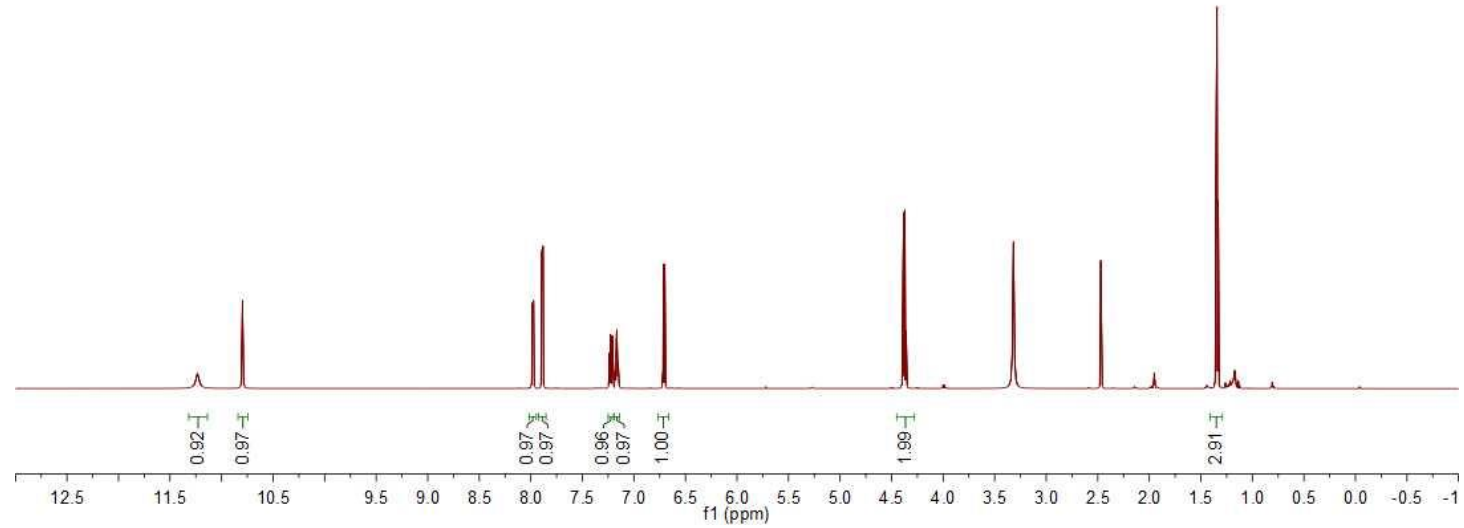

${ }^{13}$ C NMR (150 MHz, DMSO-d6)

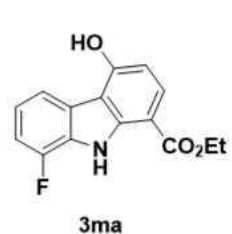

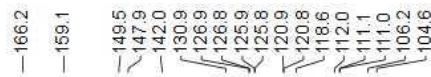

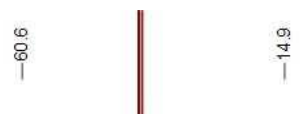

$3 \mathrm{ma}$

$\begin{array}{llllllllllll}230 & 220 & 210 & 200 & 190 & 180 & 170 & 160 & 150 & 140 & 130 & 120 \\ \mathrm{f} 1(\mathrm{ppm}) & 100\end{array}$ 
${ }^{1}$ H NMR (500 MHz, DMSO-d6)

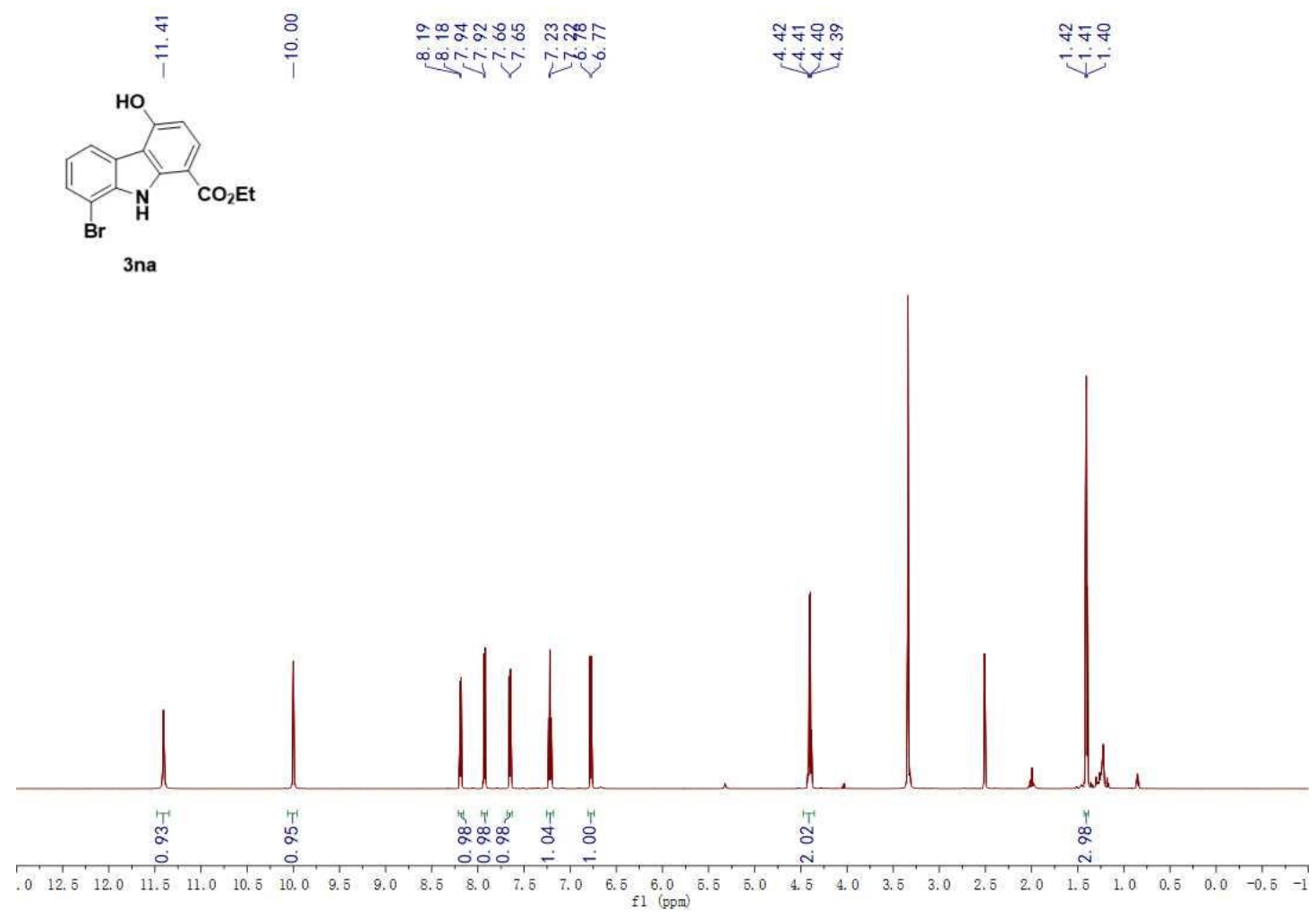

${ }^{13}$ C NMR (125 MHz, DMSO-d6)

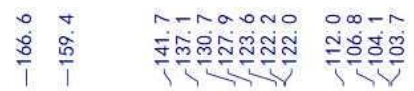

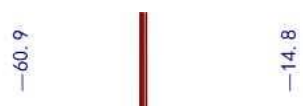
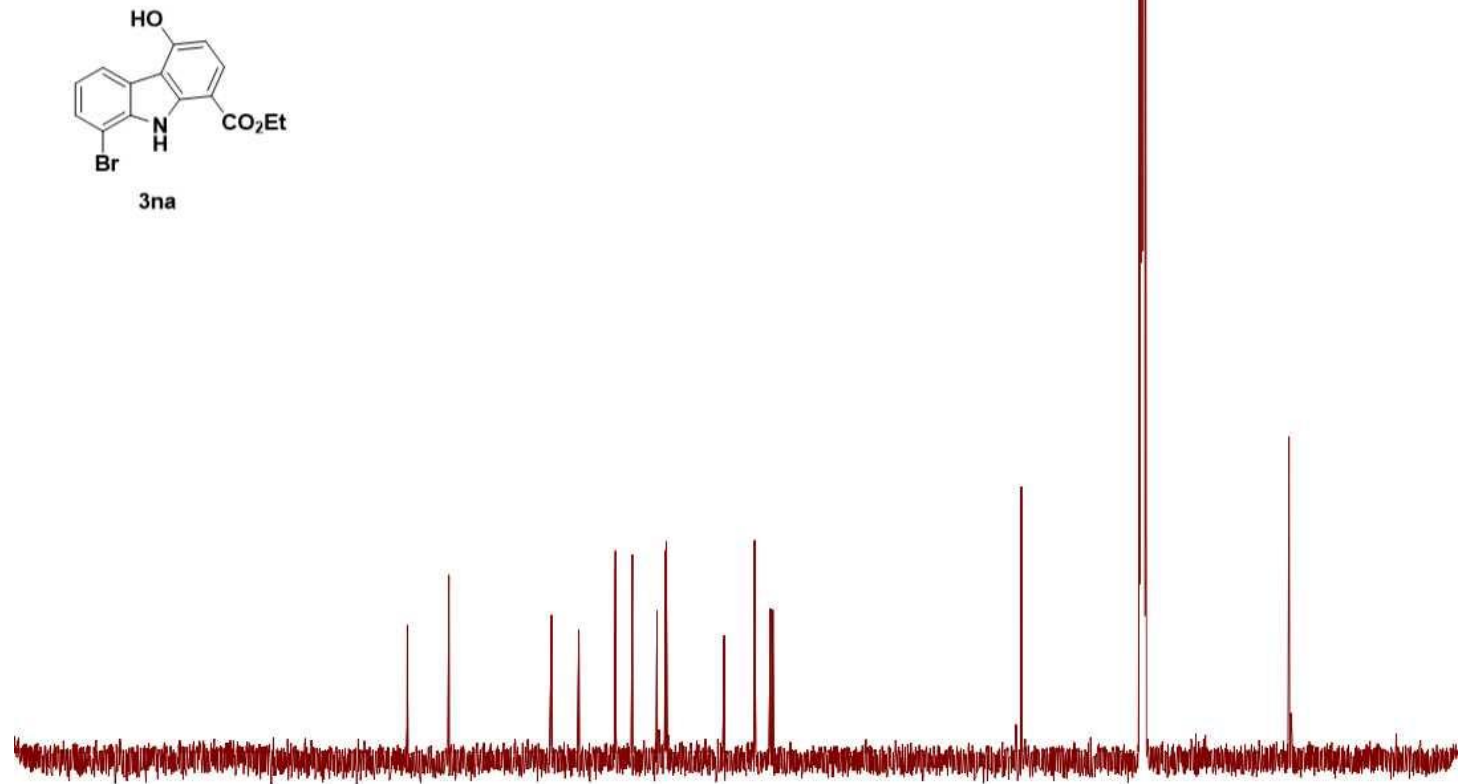

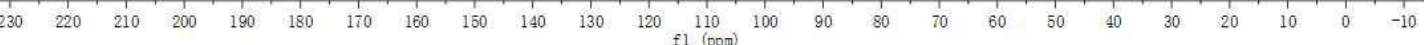


${ }^{1}$ H NMR (600 MHz, DMSO-d6)

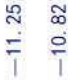

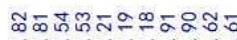

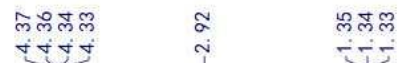
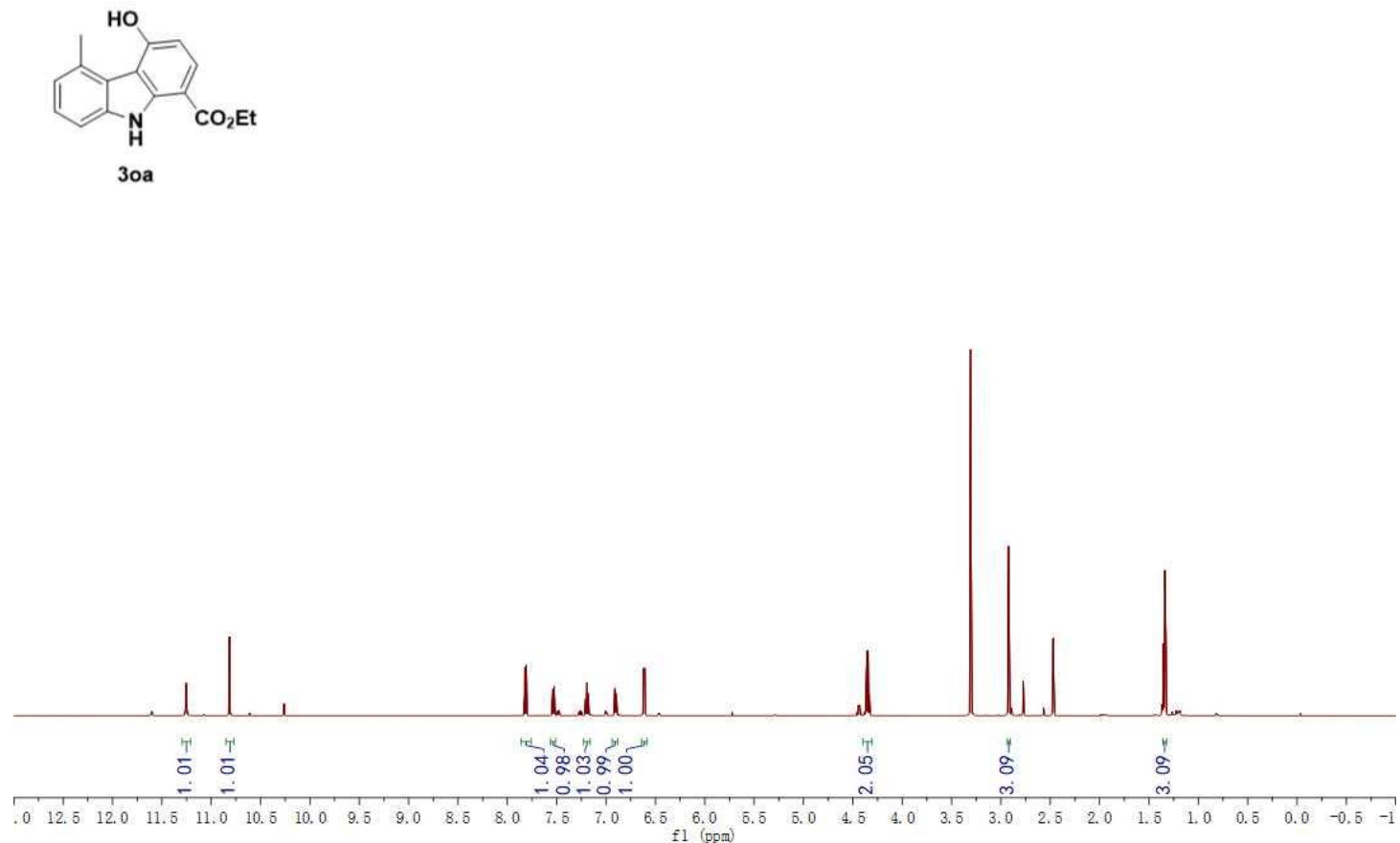

${ }^{13}$ C NMR (150 MHz, DMSO-d6)

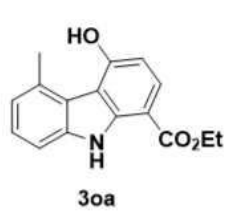

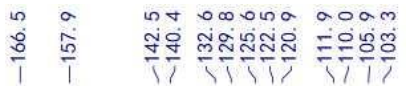

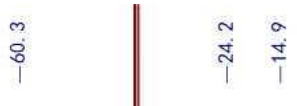

3oa

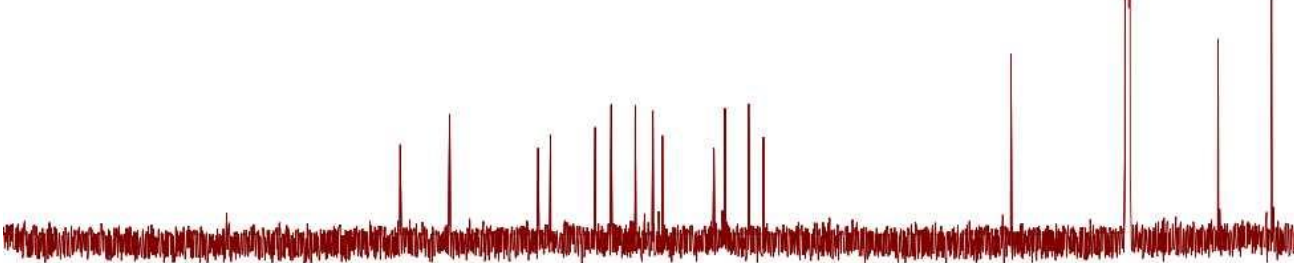

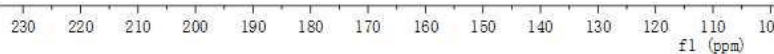


${ }^{1}$ H NMR (500 MHz, DMSO-d6)

$\stackrel{\overline{1}}{\overline{1}}$
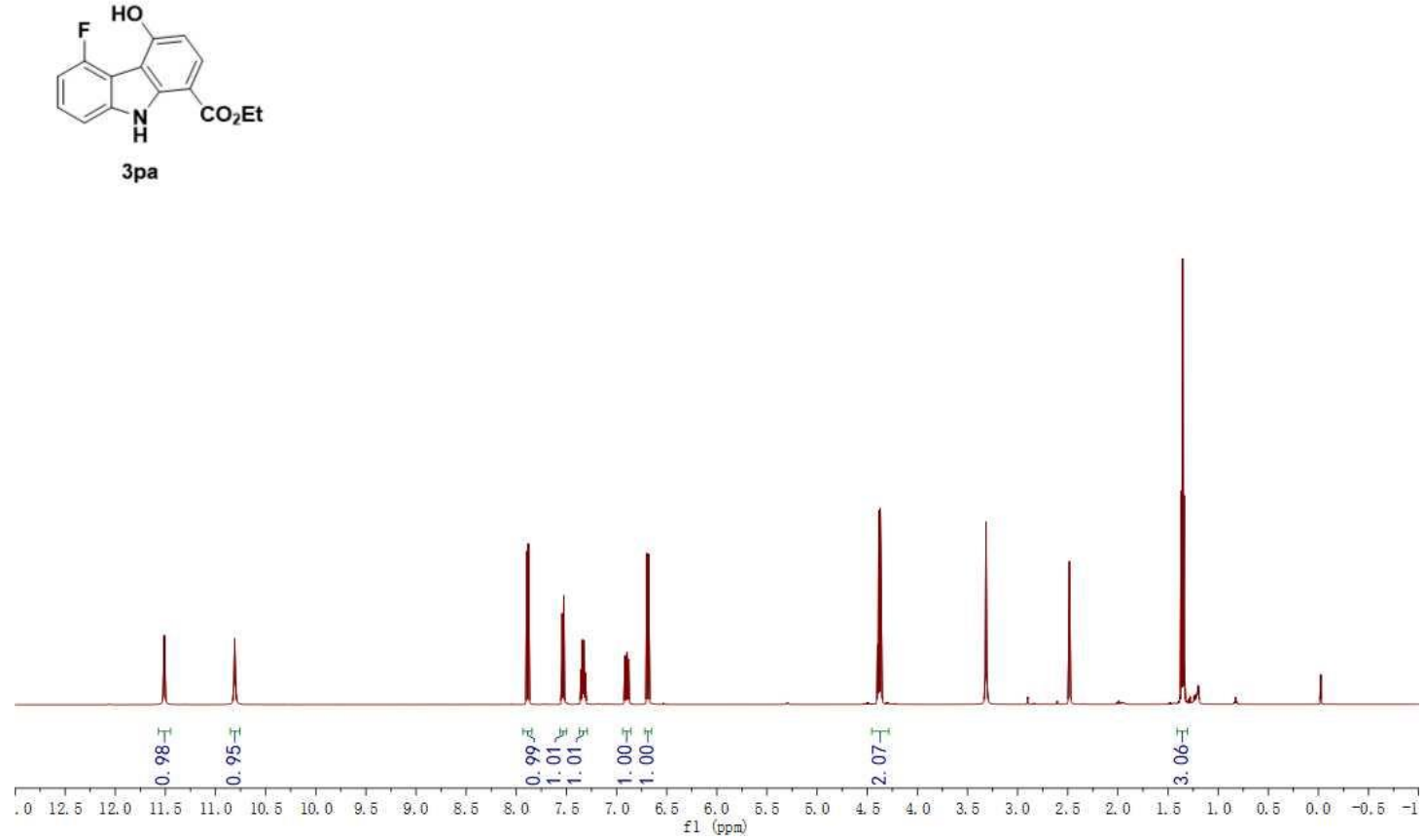

${ }^{13}$ C NMR (125 MHz, DMSO-d6)

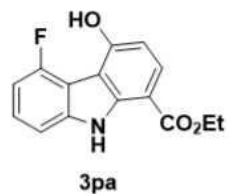

- 00 nN- nod - non

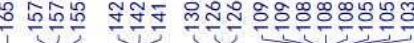

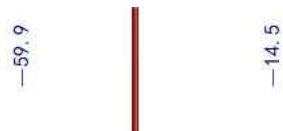

$3 \mathrm{pa}$

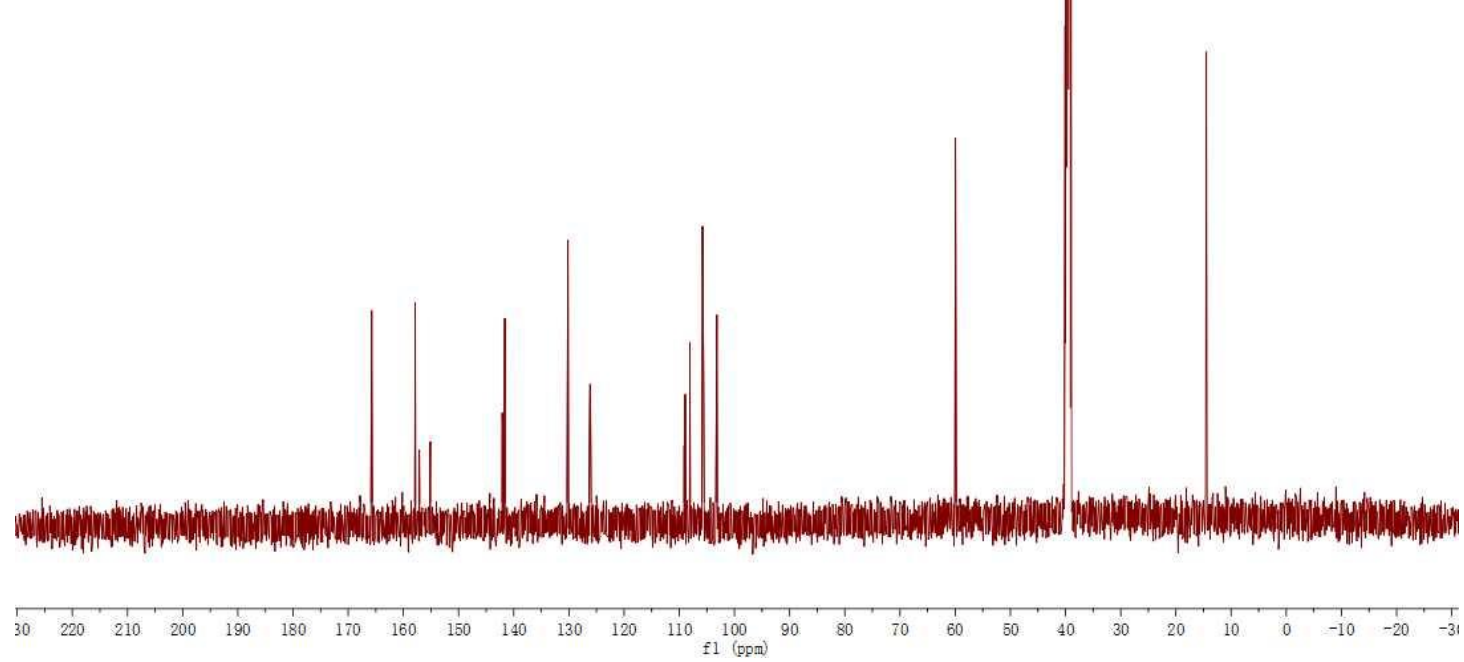


${ }^{1}$ H NMR (500 MHz, DMSO-d6)
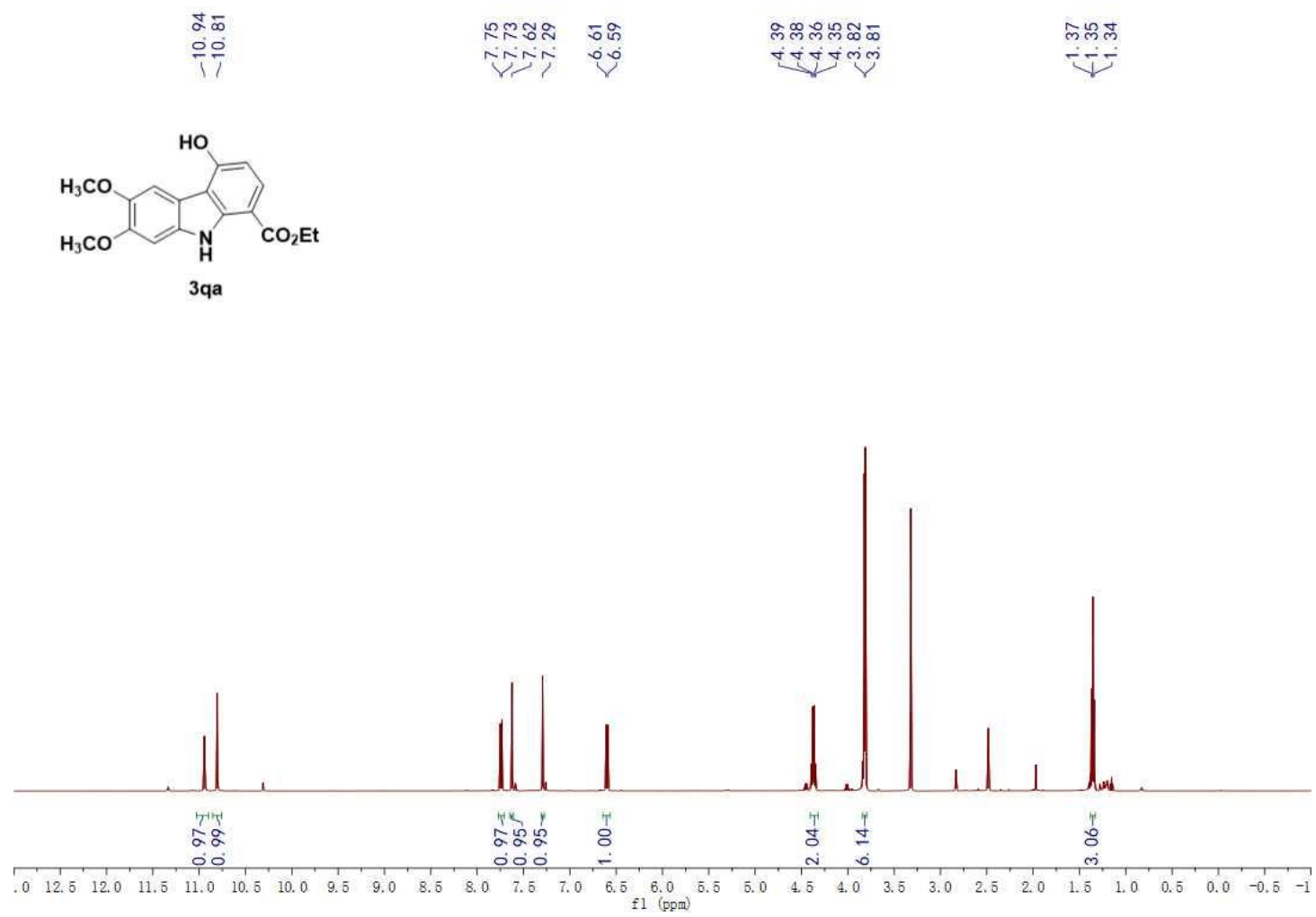

${ }^{13}$ C NMR (125 MHz, DMSO-d6)
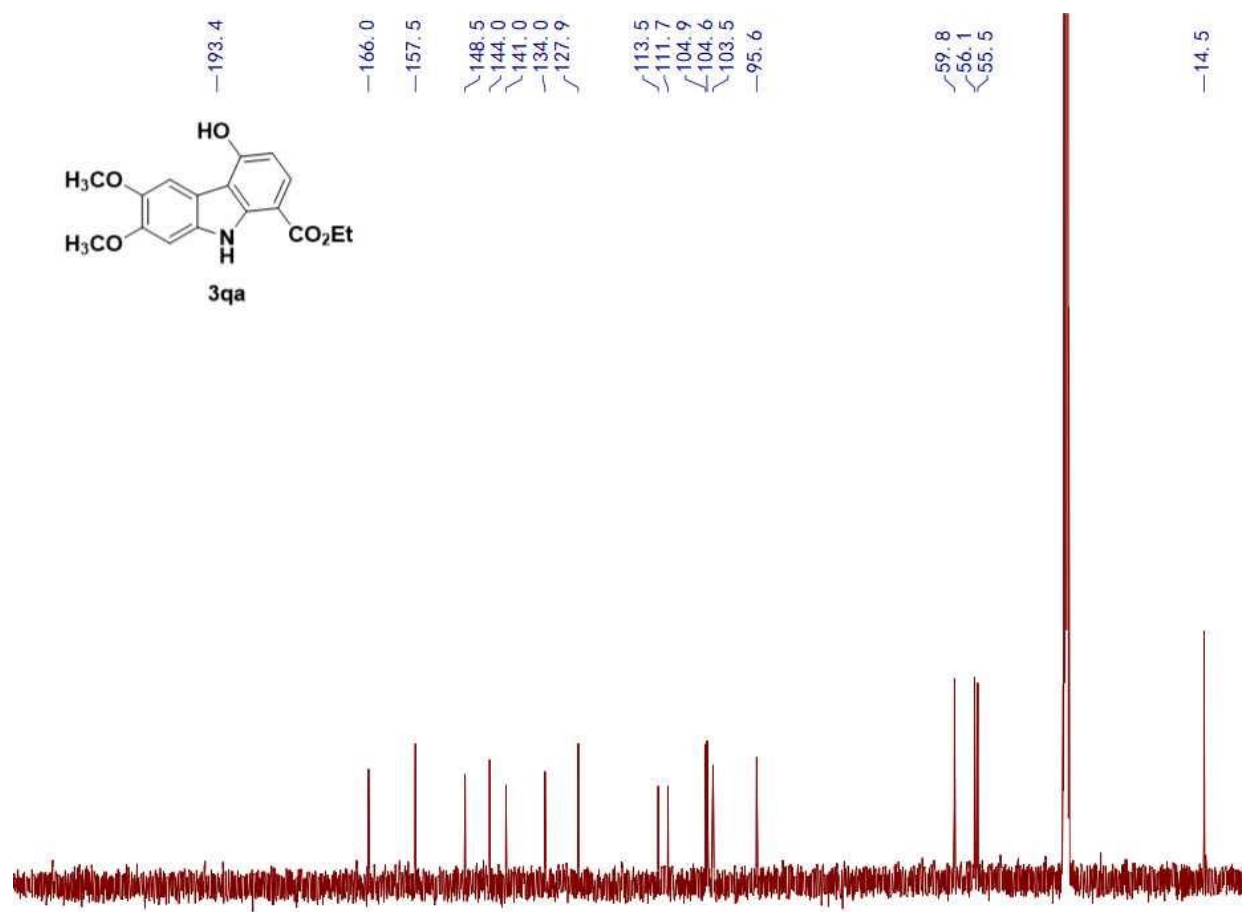

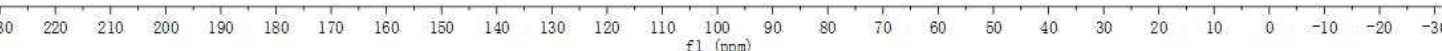


${ }^{1}$ H NMR (500 MHz, DMSO-d6)

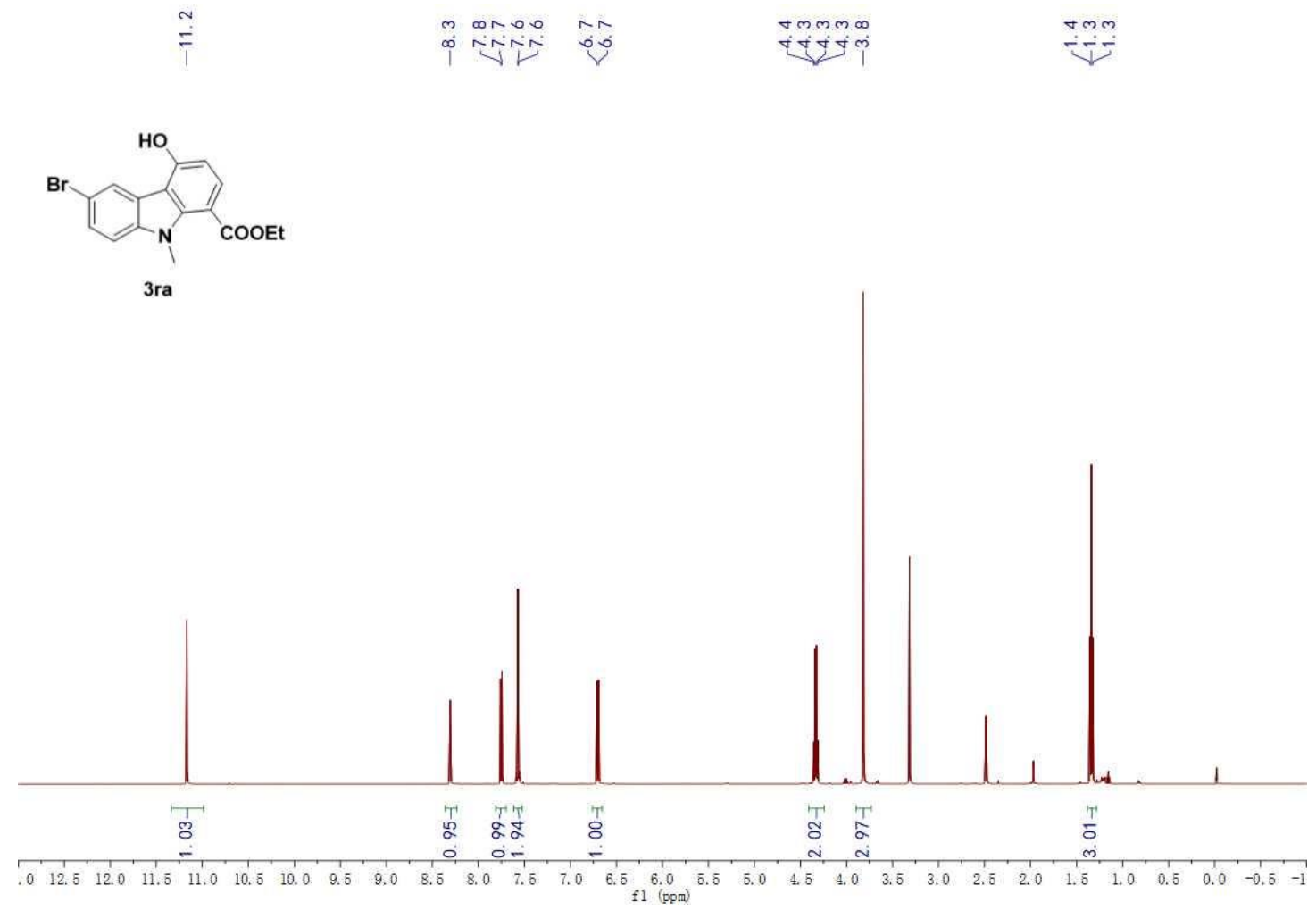

${ }^{13}$ C NMR (125 MHz, DMSO-d6)
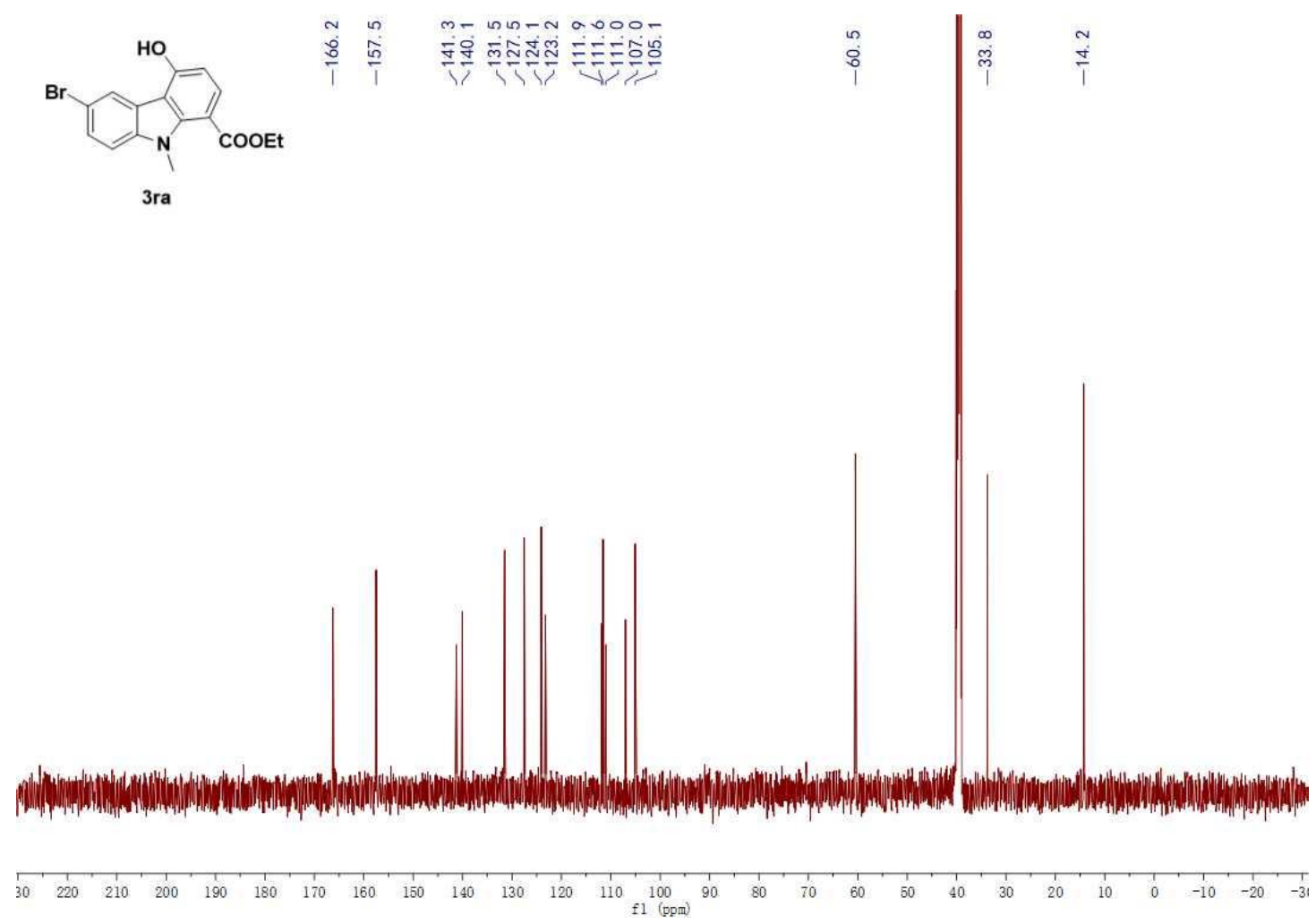
${ }^{1}$ H NMR (500 MHz, DMSO-d6)
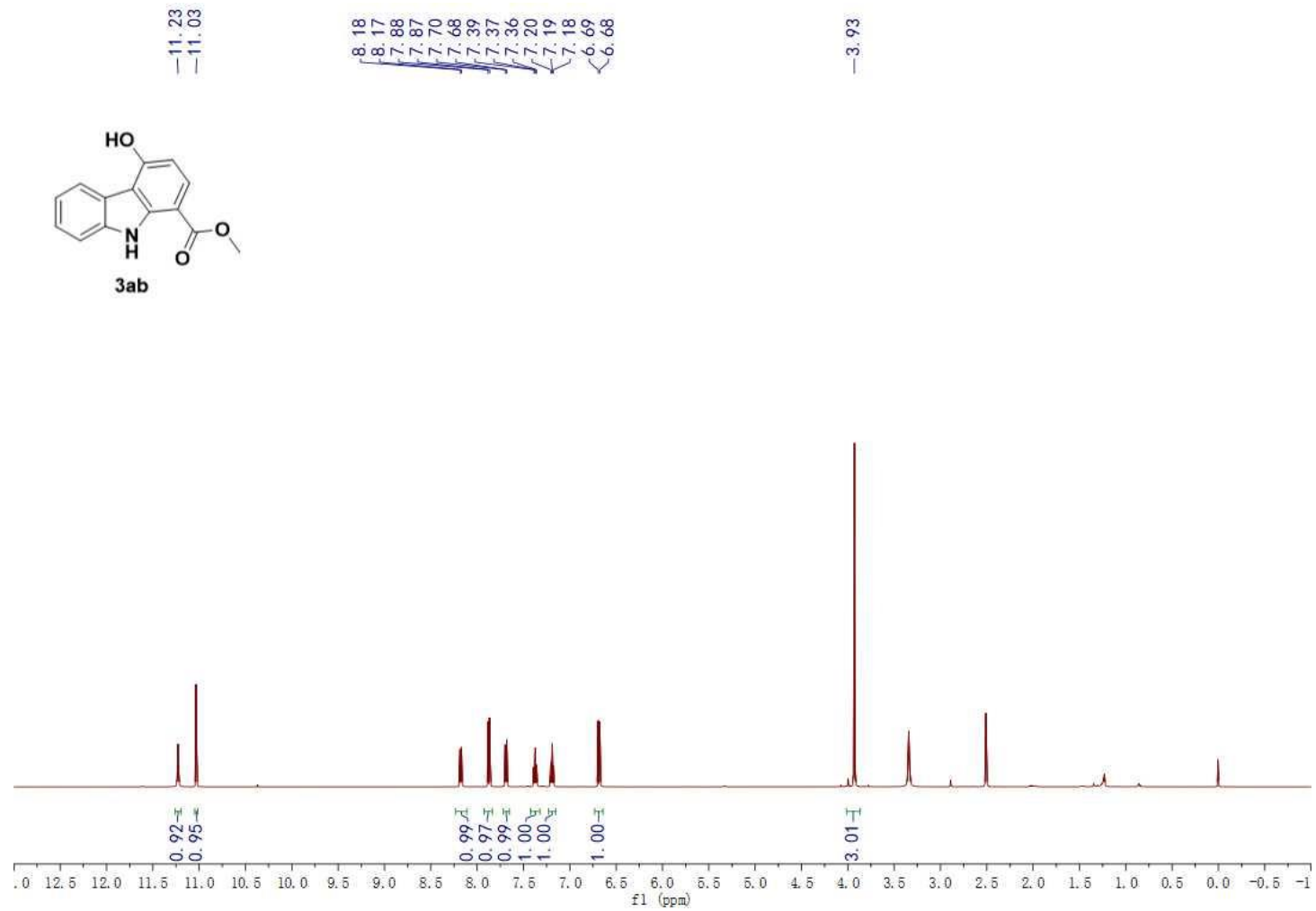

${ }^{13}$ C NMR (125 MHz, DMSO-d6)

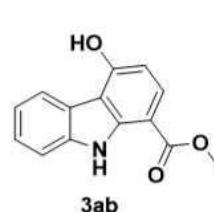

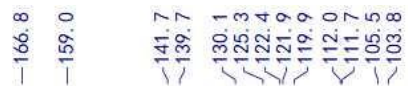

$\frac{9}{1}$

3ab

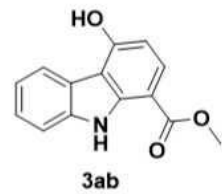

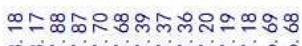

onninininino 
${ }^{1}$ H NMR (600 MHz, DMSO-d6)
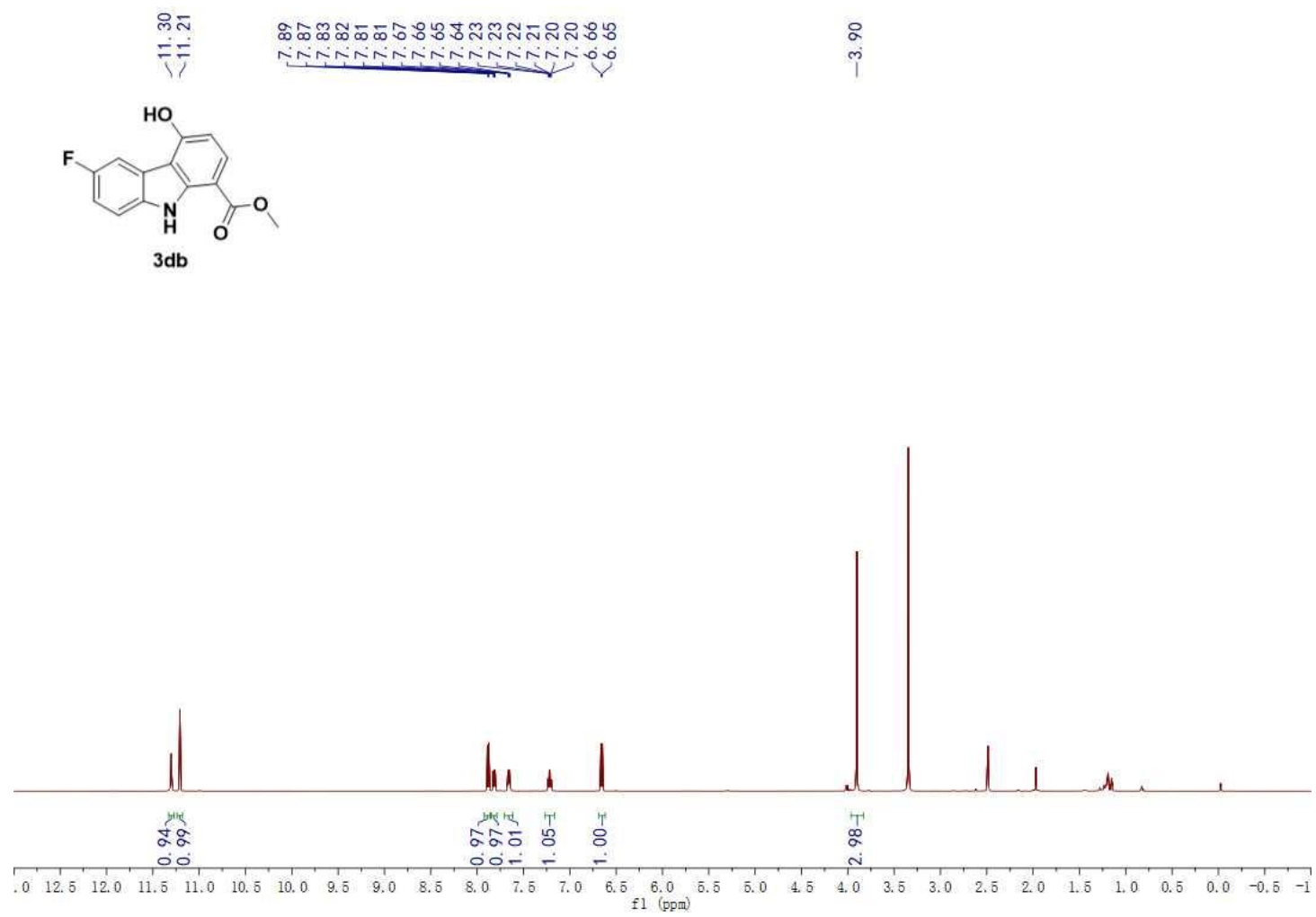

${ }^{13}$ C NMR (150 MHz, DMSO-d6)

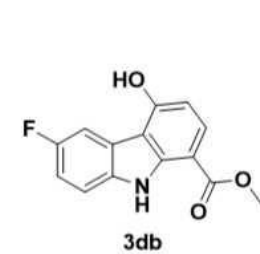

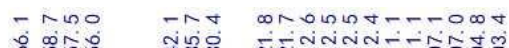

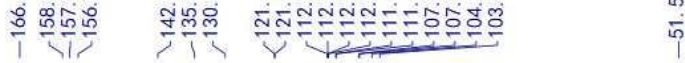

$3 \mathrm{db}$ 
${ }^{1}$ H NMR (500 MHz, DMSO-d6)
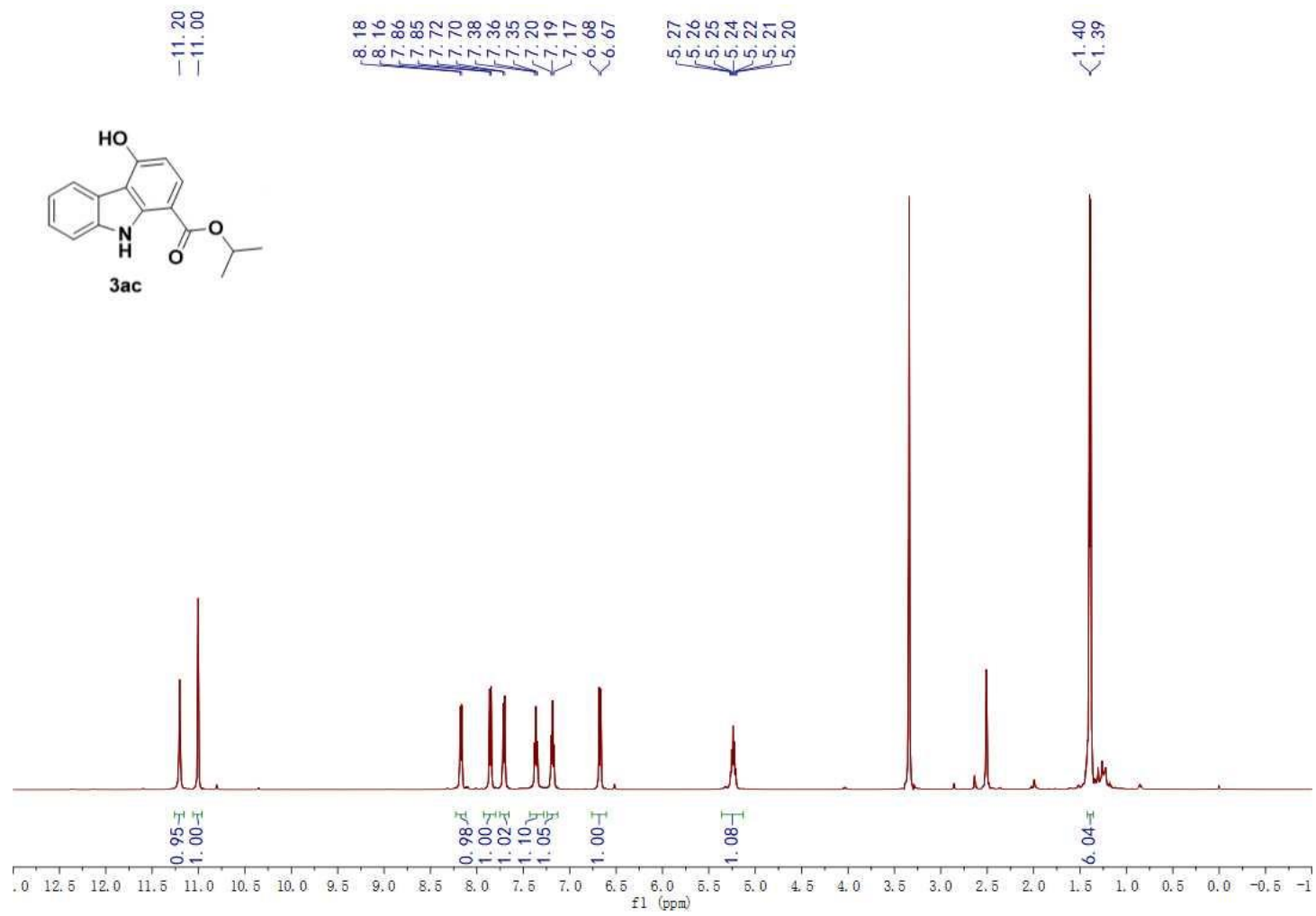

${ }^{13}$ C NMR (125 MHz, DMSO-d6)

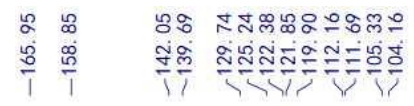

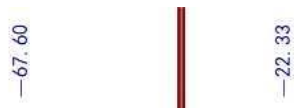
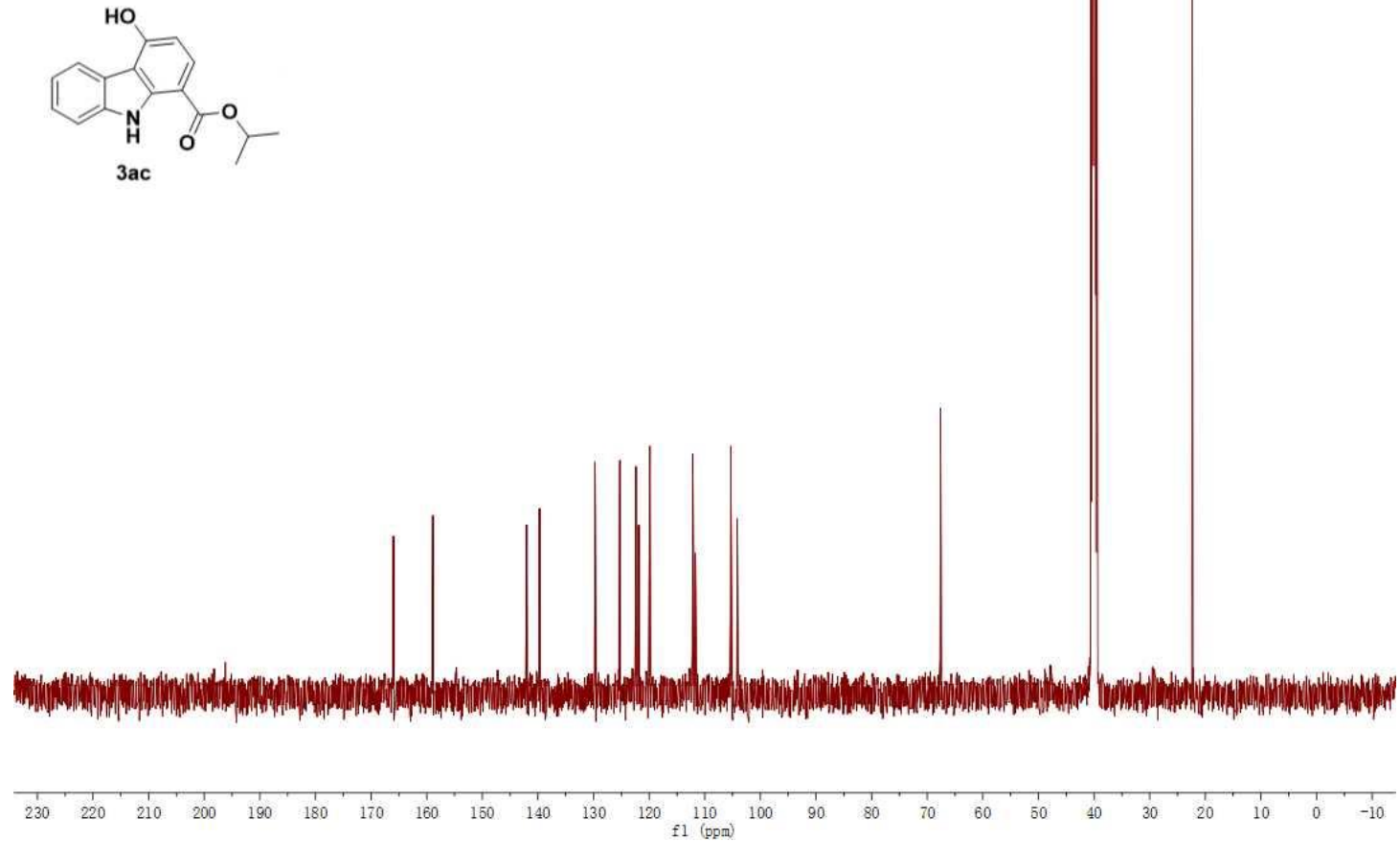
${ }^{1}$ H NMR (500 MHz, DMSO-d6)

$\underset{i=0}{i=\frac{1}{i}}$

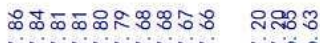

iniminiviti jió

ลกลลำ

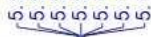

लेm

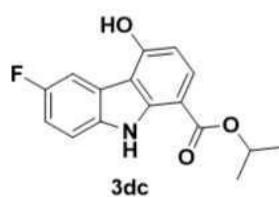

3dc

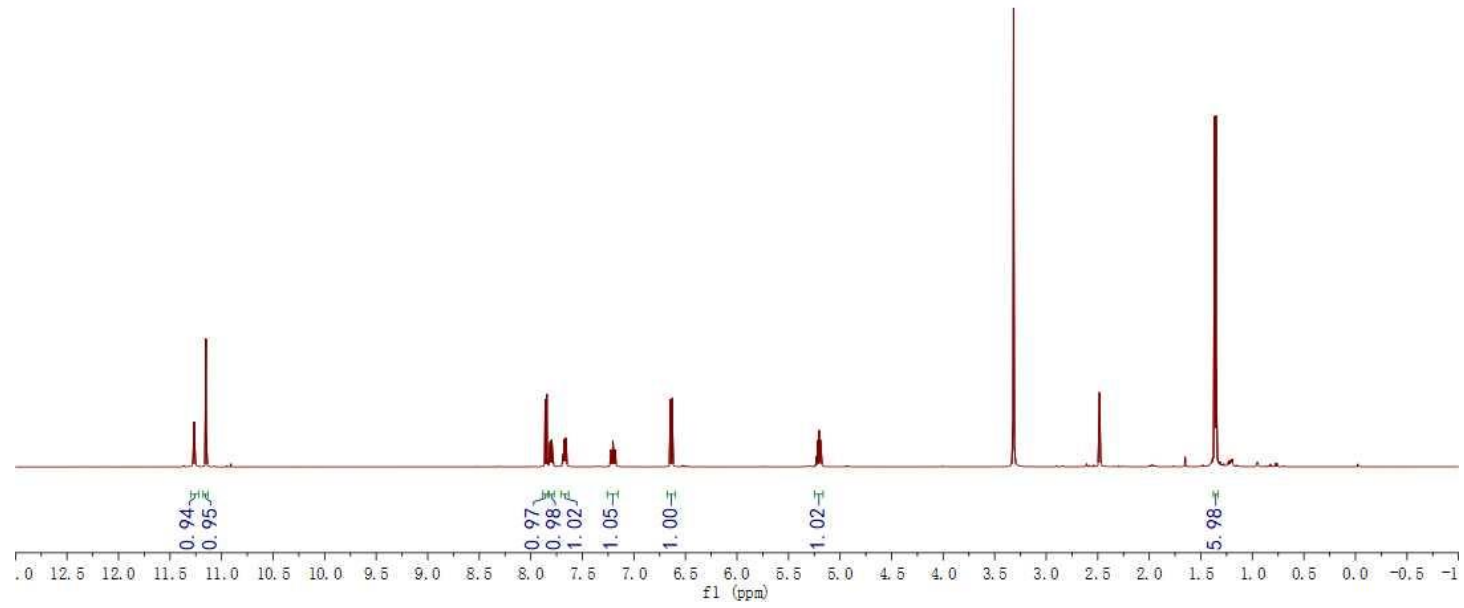

${ }^{13}$ C NMR (125 MHz, DMSO-d6)

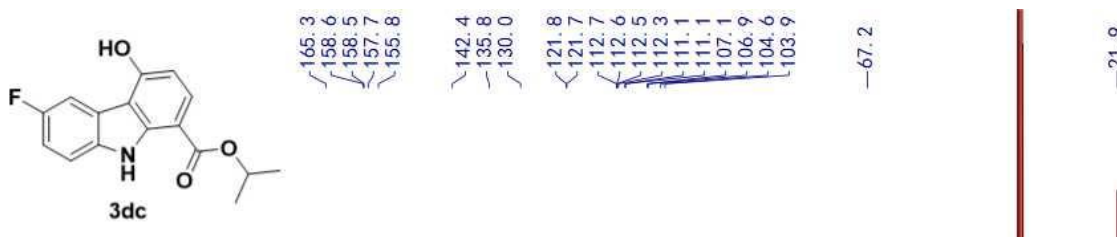

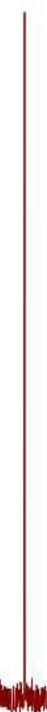

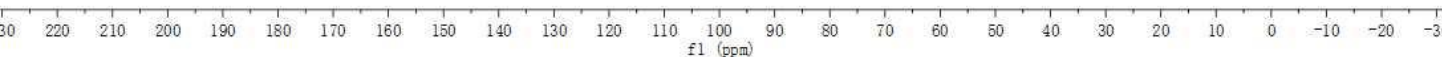


${ }^{1}$ H NMR (600 MHz, DMSO-d6)

여
$=\frac{0}{1}$

등ำㅇำ

कonitivio

มูกสกลำ

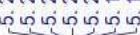

ষ

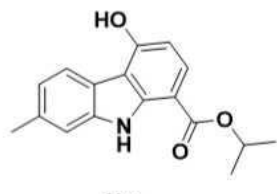

3hc

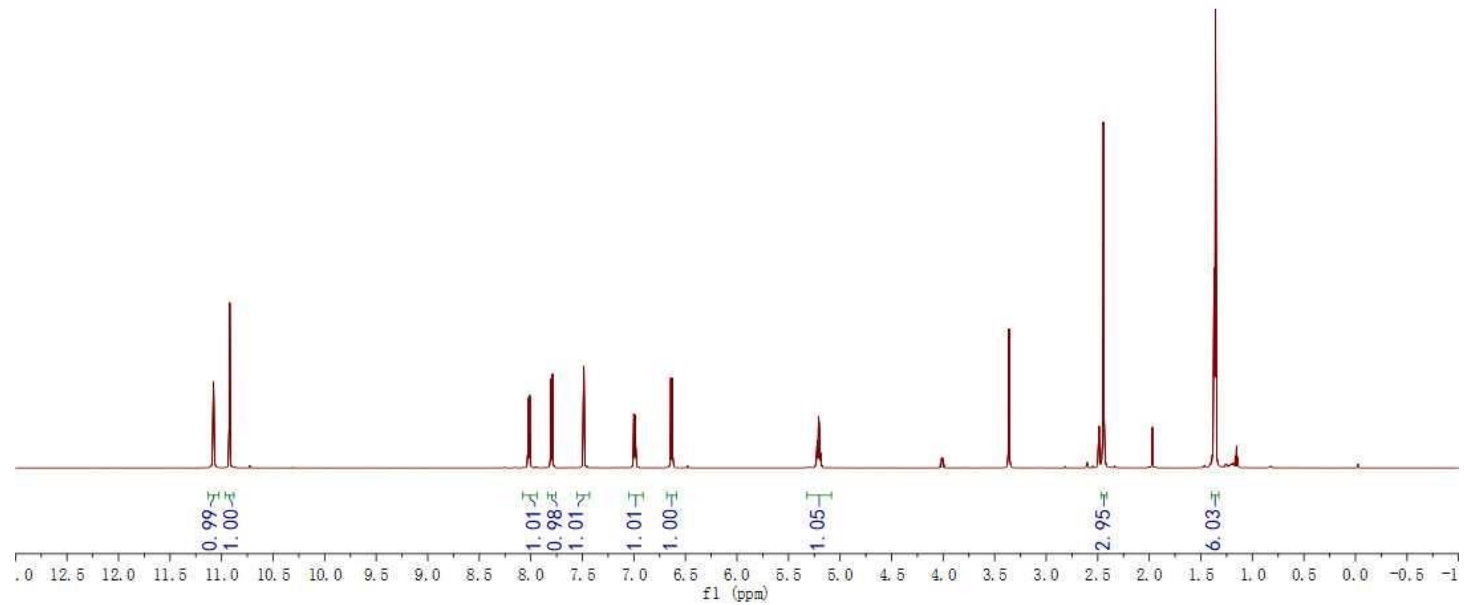

${ }^{13}$ C NMR (150 MHz, DMSO-d6)

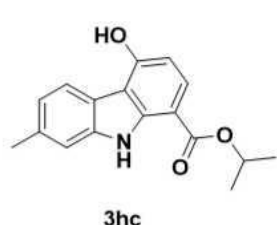

일

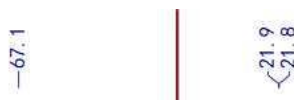

3he

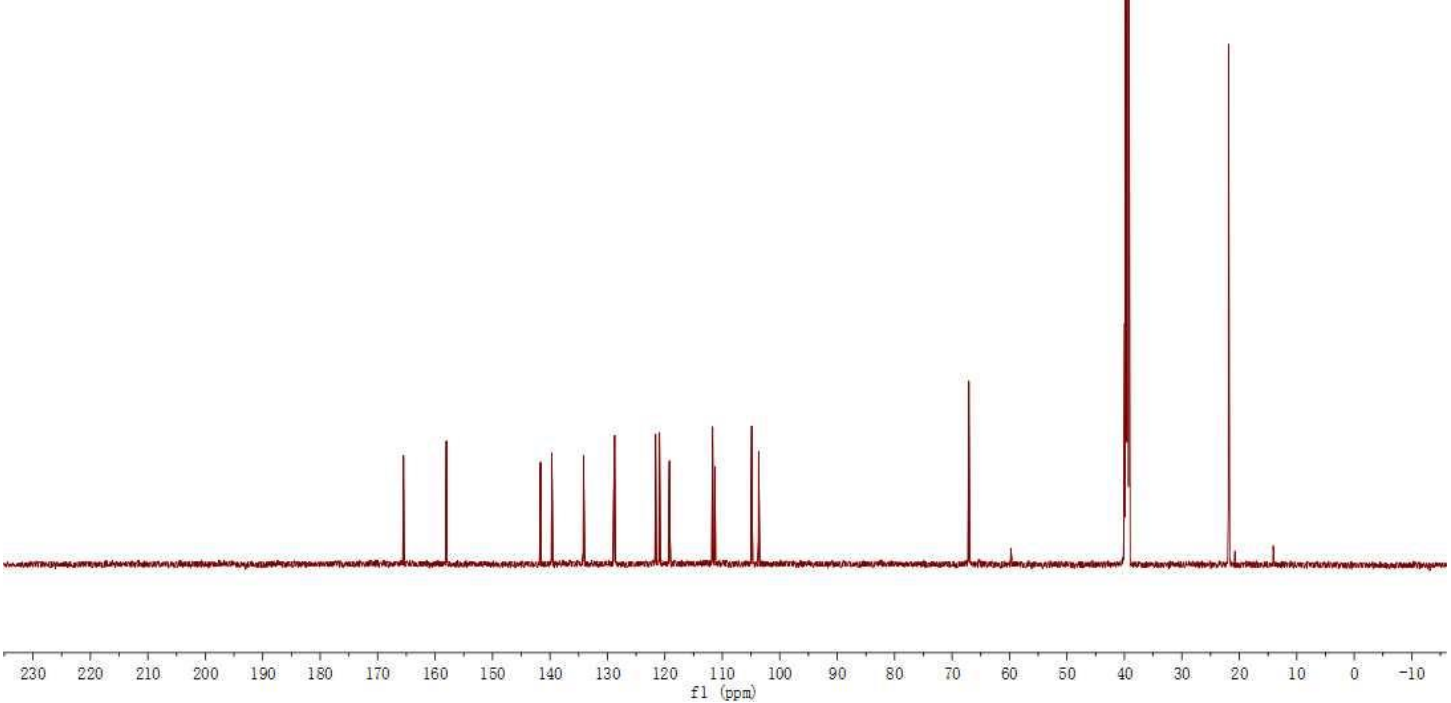


${ }^{1}$ H NMR (500 MHz, DMSO-d6)

$\stackrel{2}{2}$

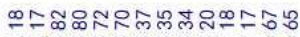

moninisinititio

$\mathrm{HO}$

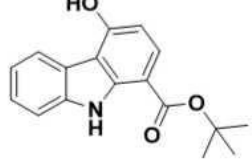

3ad

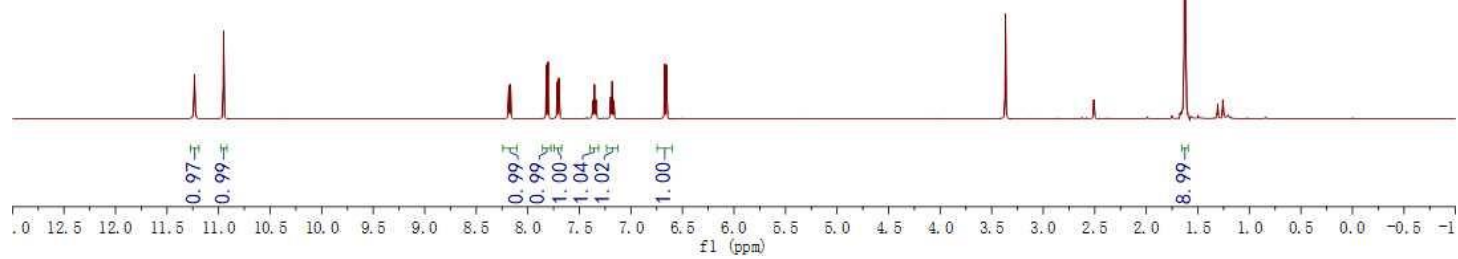

${ }^{13}$ C NMR (125 MHz, DMSO-d6)

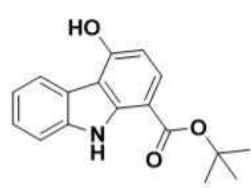

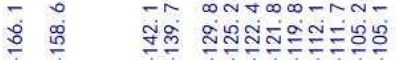

\begin{tabular}{l|l}
$\stackrel{\infty}{\infty}$ & $\stackrel{\infty}{1}$ \\
$\stackrel{\infty}{1}$ &
\end{tabular}

3ad

(1)

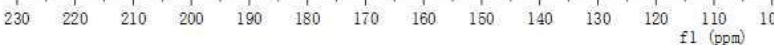


${ }^{1}$ H NMR (500 MHz, DMSO-d6)

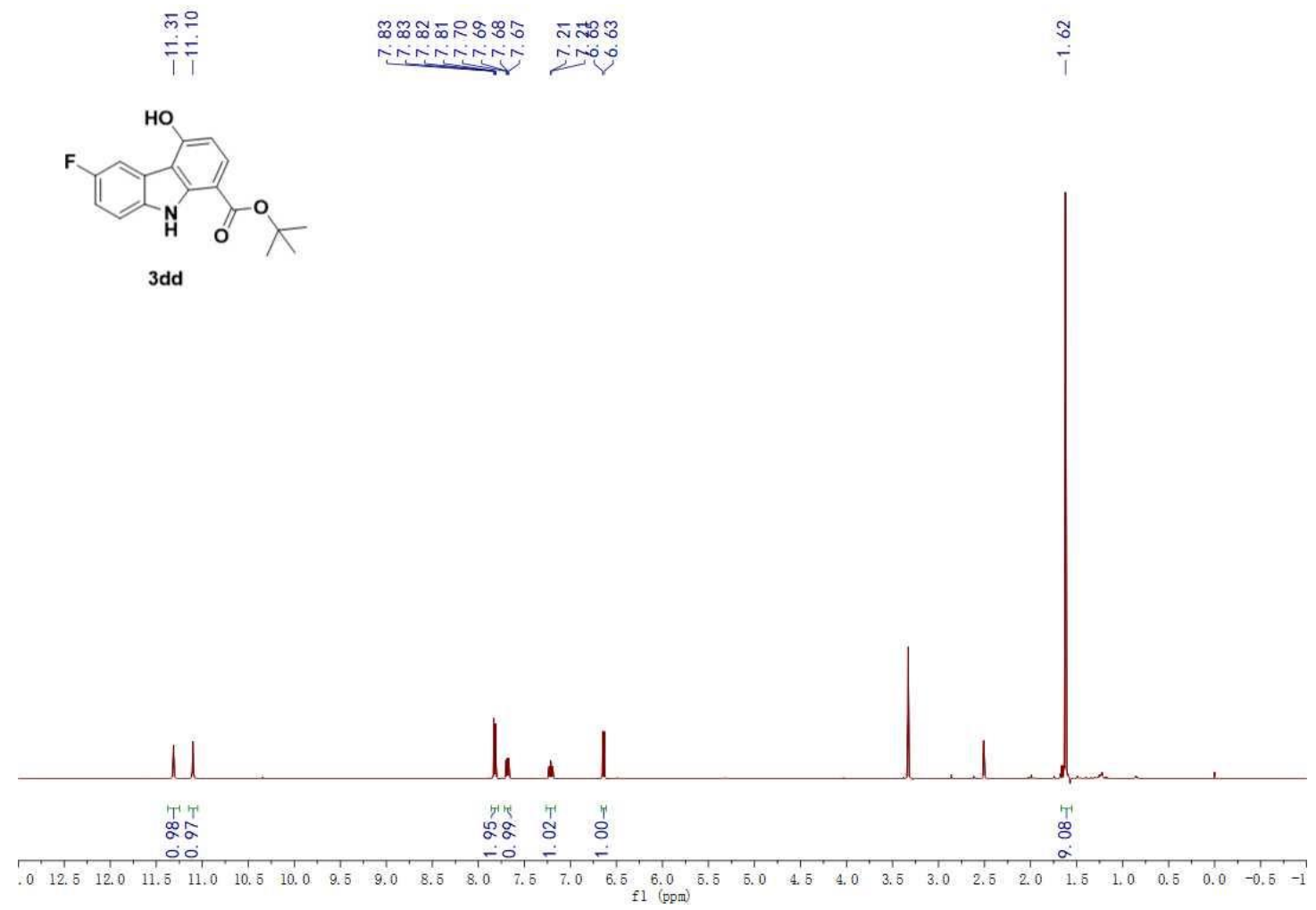

${ }^{13}$ C NMR (125 MHz, DMSO-d6)

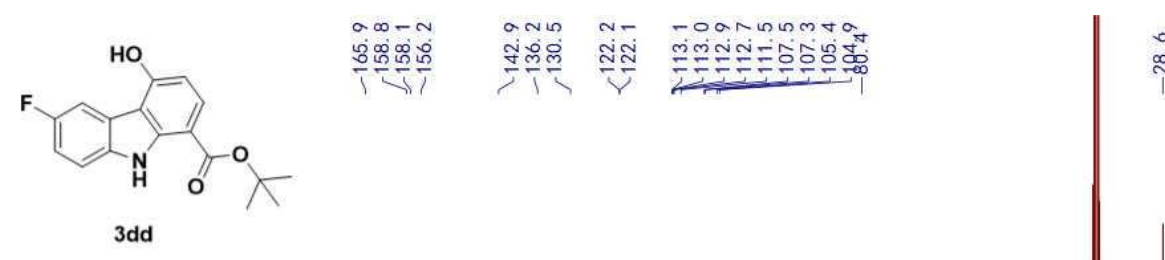

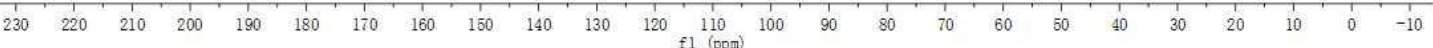


${ }^{1}$ H NMR (600 MHz, DMSO-d6)

\begin{tabular}{l}
$8 \infty$ \\
\hdashline \\
$=$ \\
1
\end{tabular}

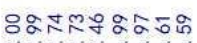

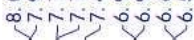

$\stackrel{i}{i} \frac{1}{4}$

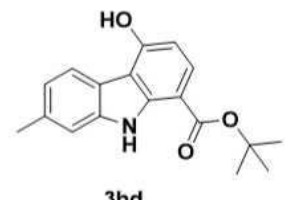

3hd

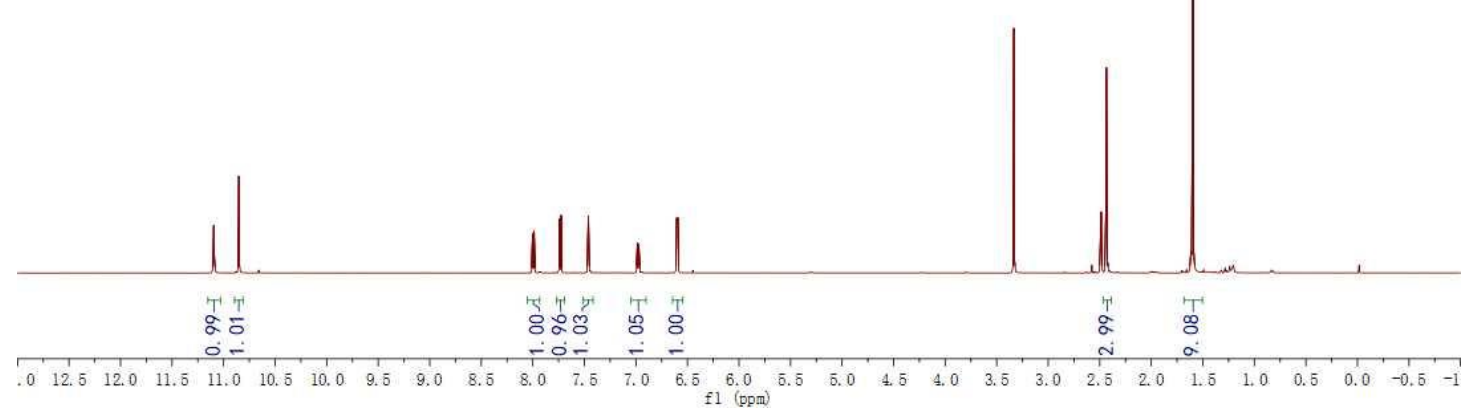

${ }^{13}$ C NMR (150 MHz, DMSO-d6)

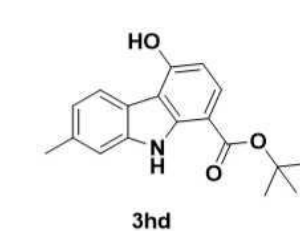

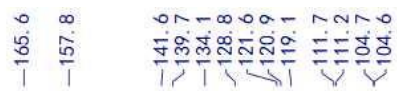

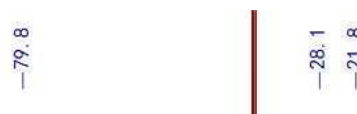

3hd

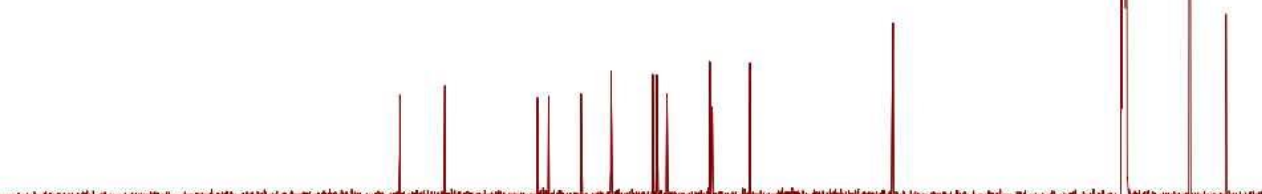

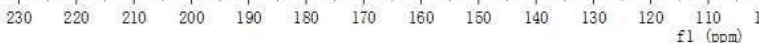


${ }^{1}$ H NMR (500 MHz, DMSO-d6)

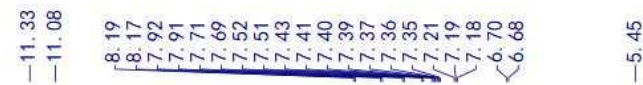
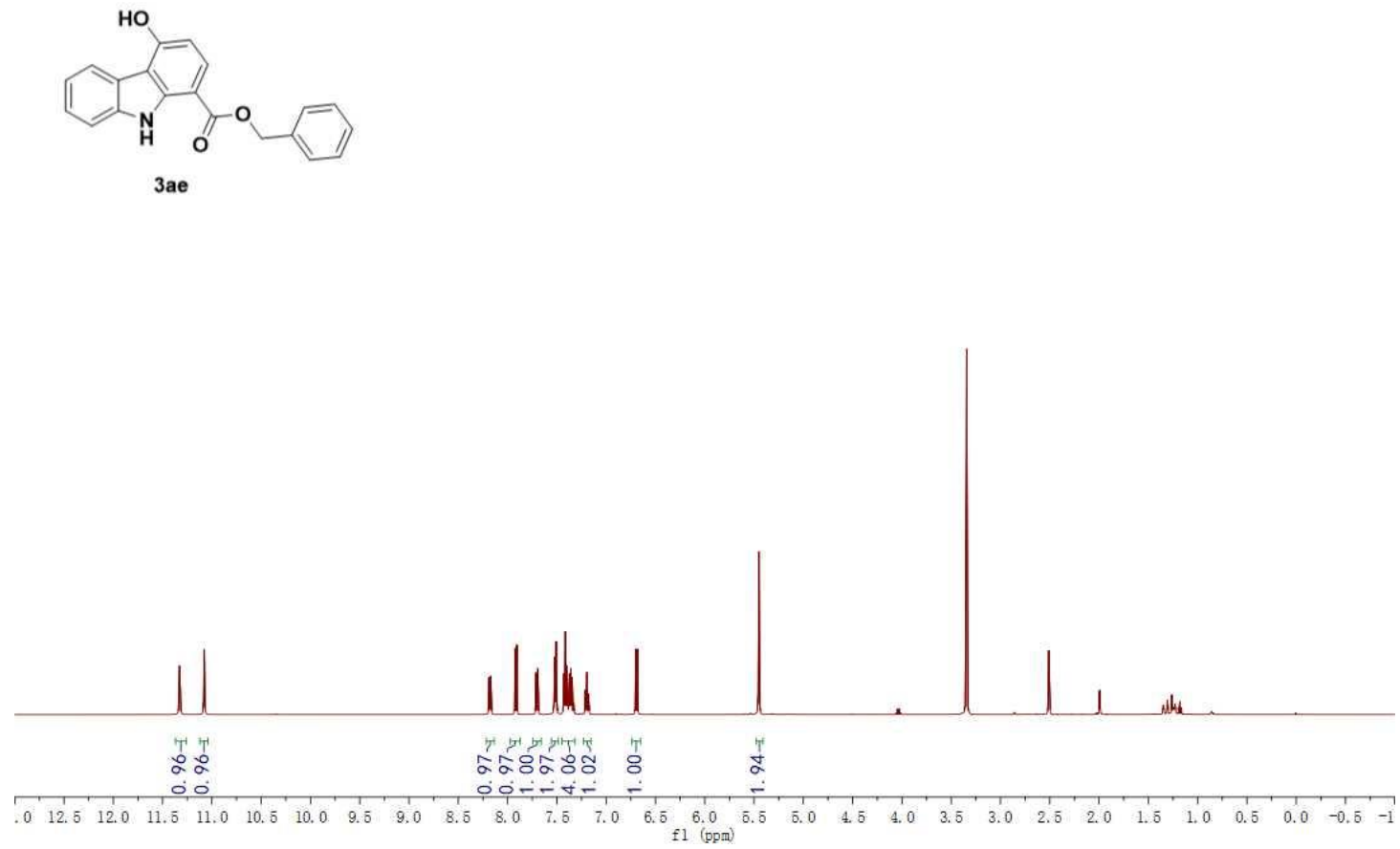

${ }^{13}$ C NMR (125 MHz, DMSO-d6)
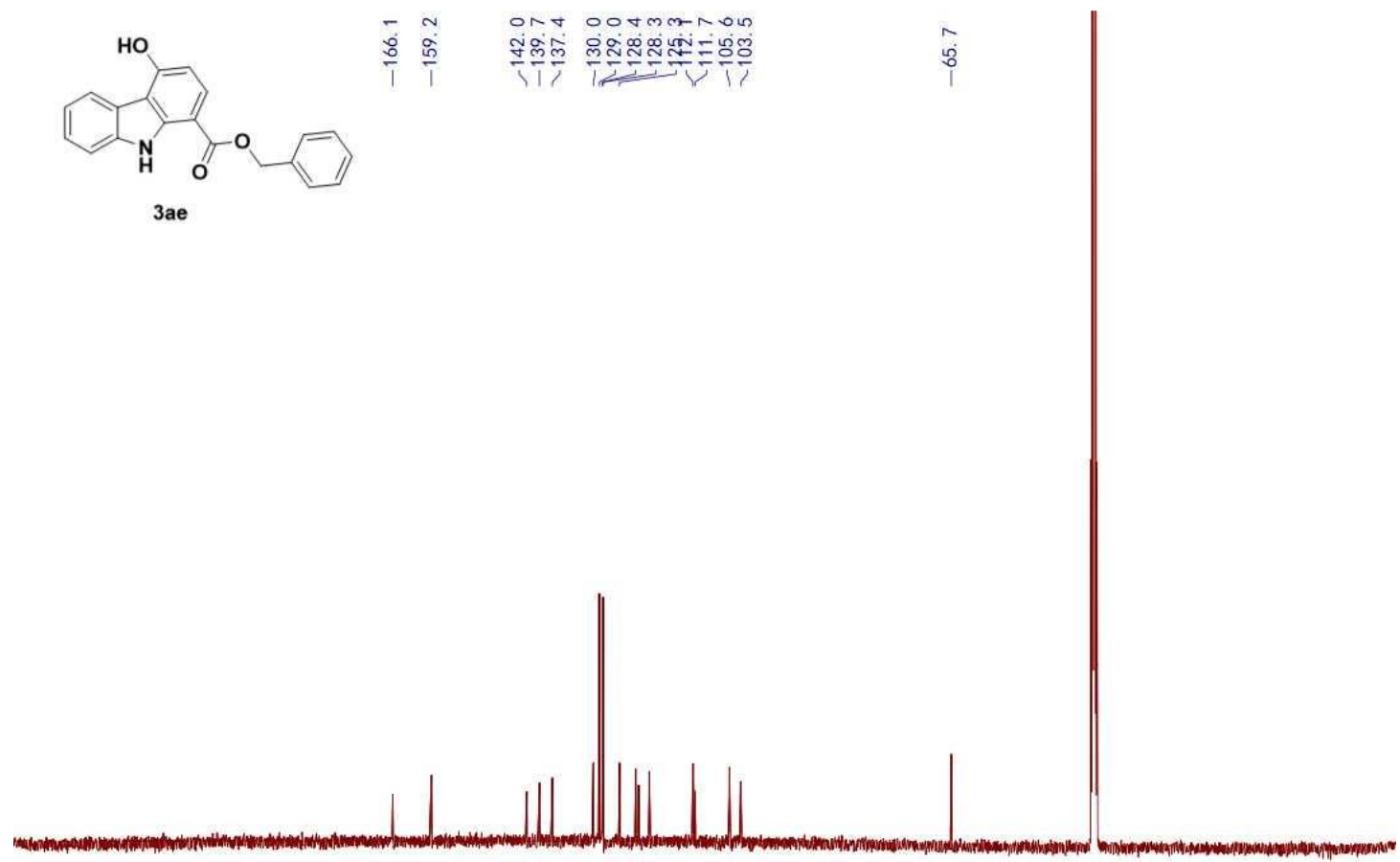

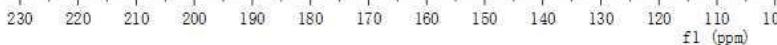


${ }^{1}$ H NMR (500 MHz, DMSO-d6)

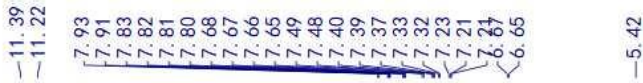

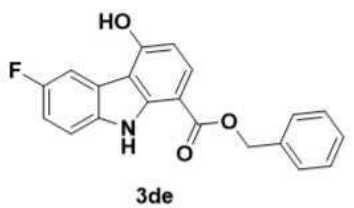

3de

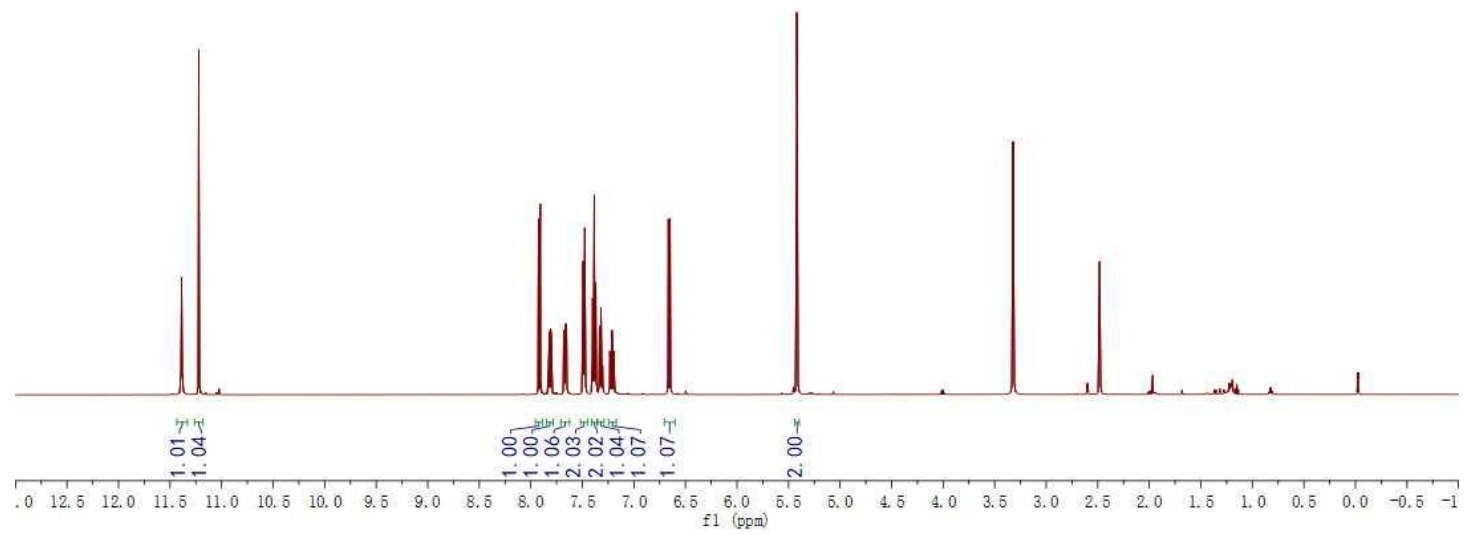

${ }^{13}$ C NMR (125 MHz, DMSO-d6)

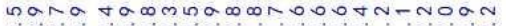

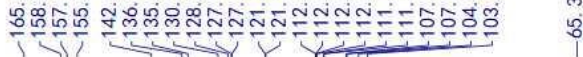

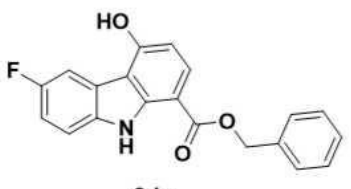

3de

\section{1
mi}


${ }^{1}$ H NMR (500 MHz, DMSO-d6)

$\infty$
$=\infty$
$=0$

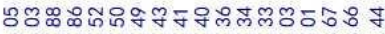

oninininininininiviog of

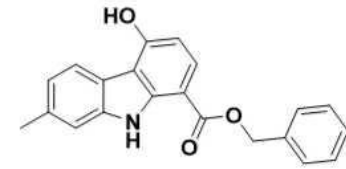

3he

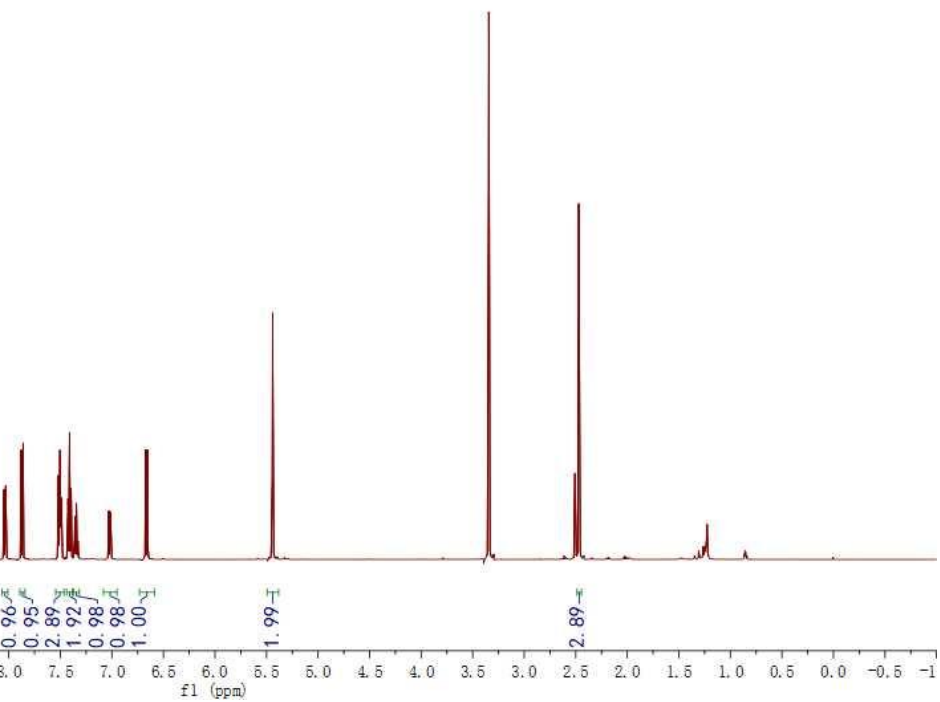

${ }^{13}$ C NMR (125 MHz, DMSO-d6)
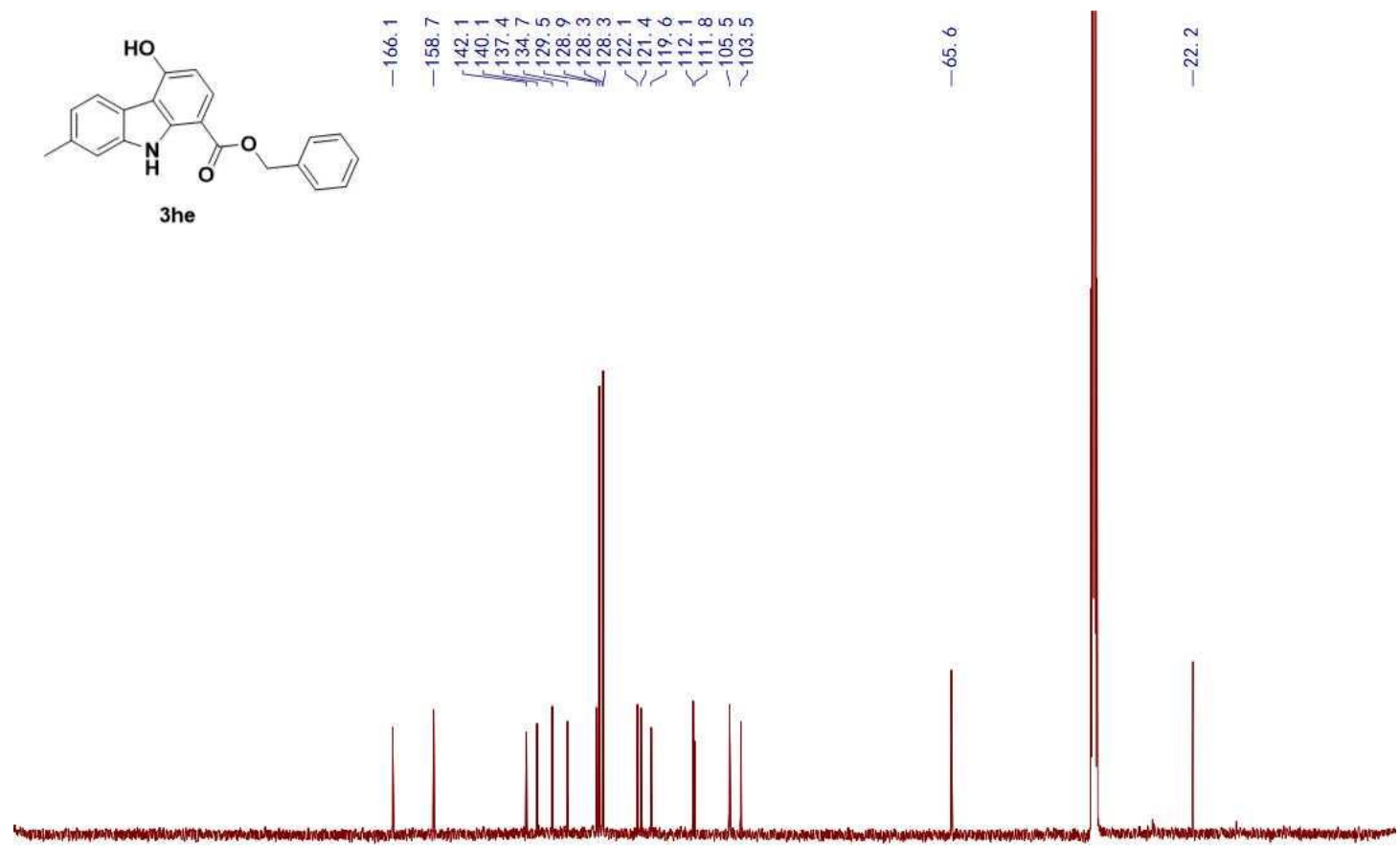

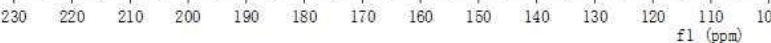


${ }^{1}$ H NMR (500 MHz, DMSO-d6)

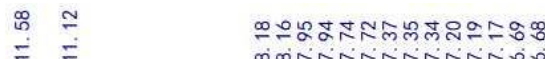

monitinitivinitio

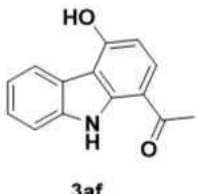

3af

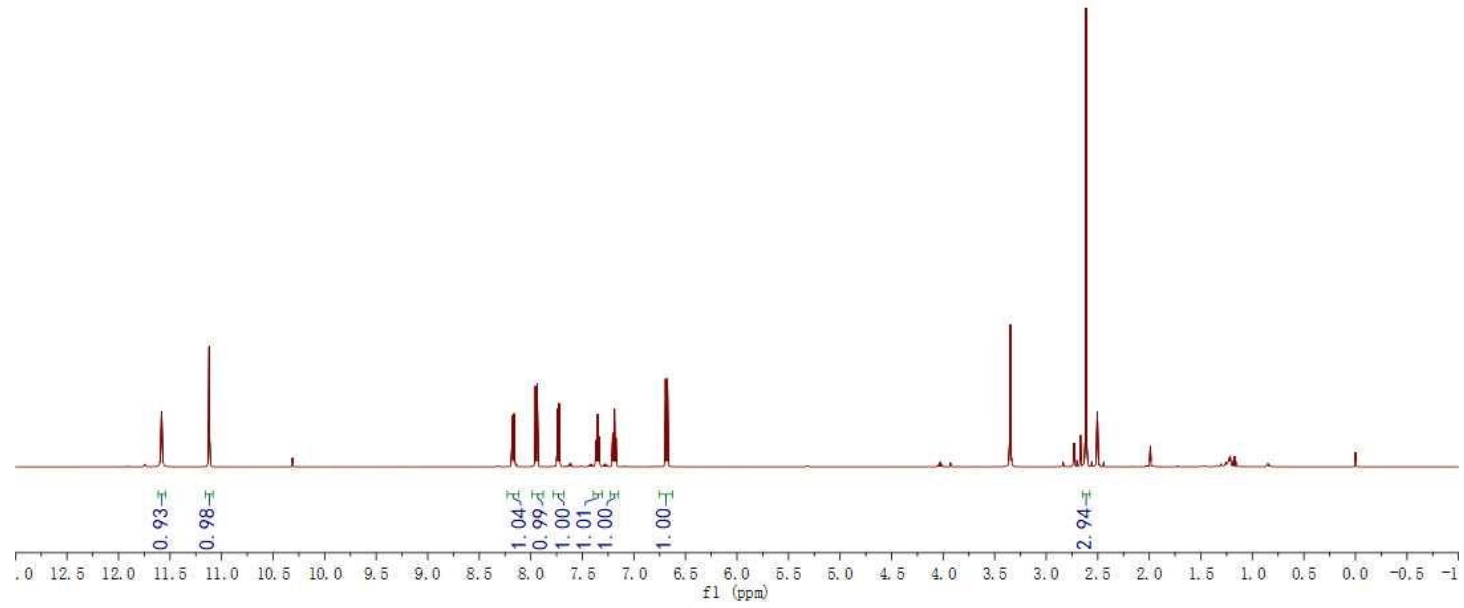

${ }^{13}$ C NMR (125 MHz, DMSO-d6)

$\begin{array}{lll}\infty & \infty & 0 m-N \infty 000000 \\ \stackrel{0}{l} & \infty\end{array}$

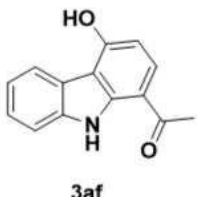

3af

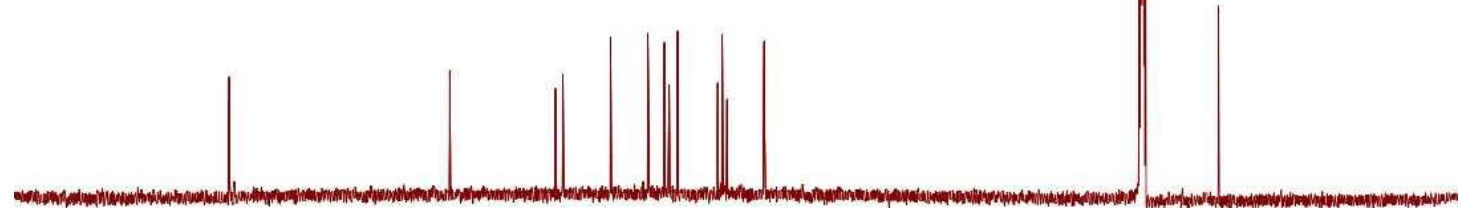

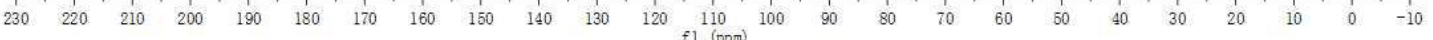


${ }^{1}$ H NMR (500 MHz, DMSO-d6)

7
$\overline{1}$
$\overline{1}$

HO

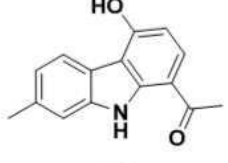

$3 d f$

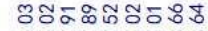

initivio

82

พิ

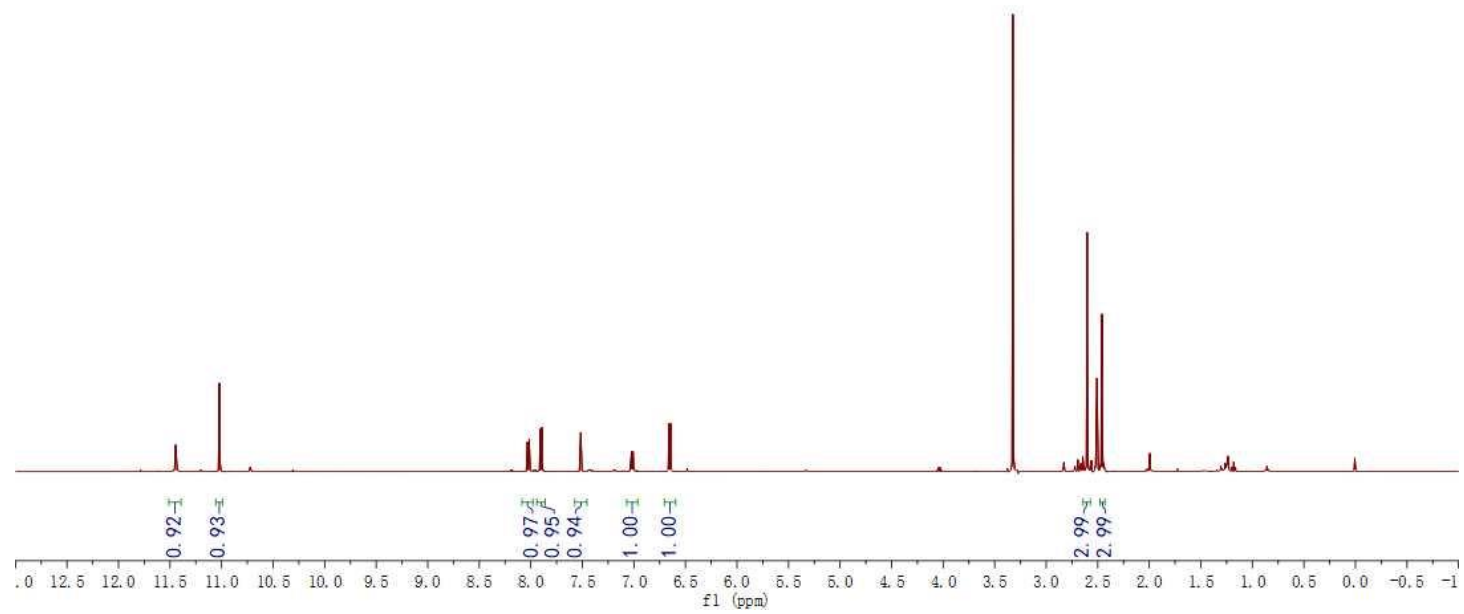

${ }^{13}$ C NMR (125 MHz, DMSO-d6)

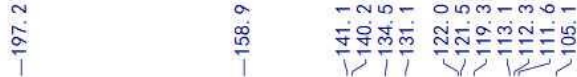

กิ่

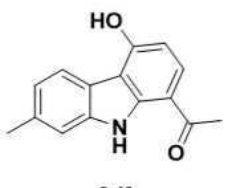

3df

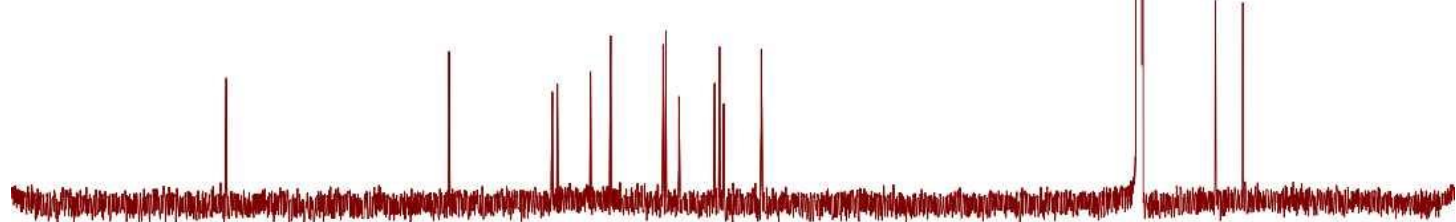

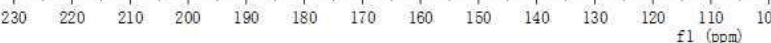


${ }^{1}$ H NMR (500 MHz, DMSO-d6)

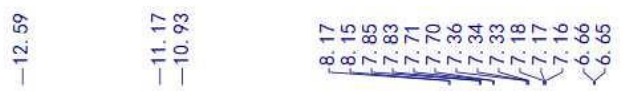
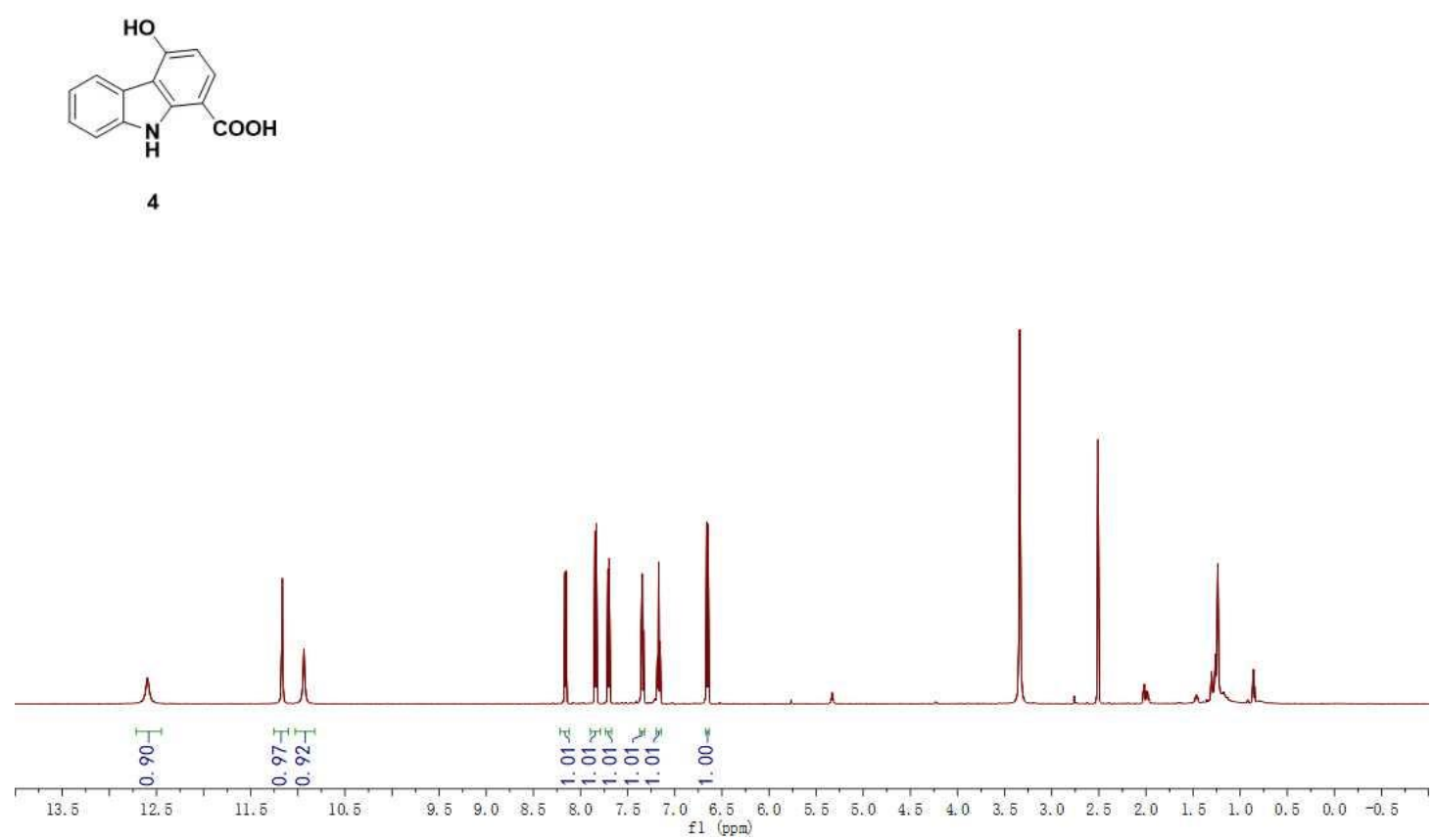

${ }^{13}$ C NMR (125 MHz, DMSO-d6)

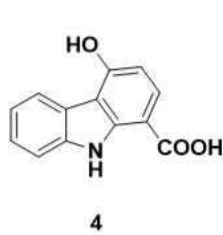

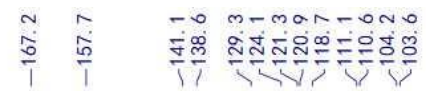

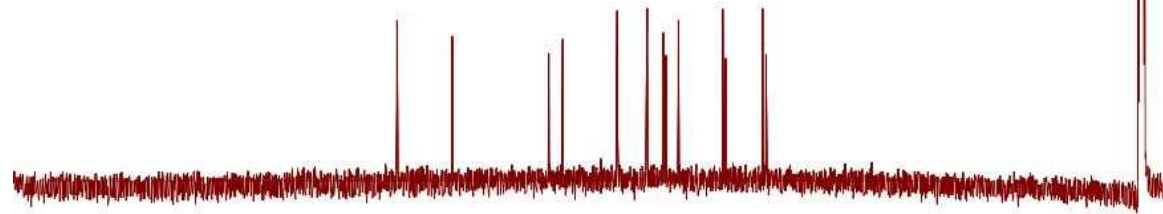

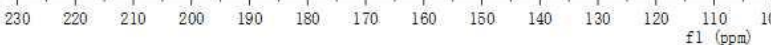


${ }^{1}$ H NMR (500 MHz, DMSO-d6)

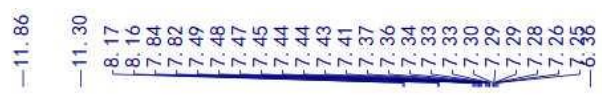

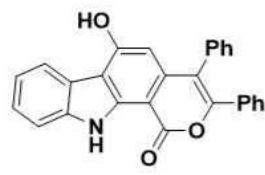

5

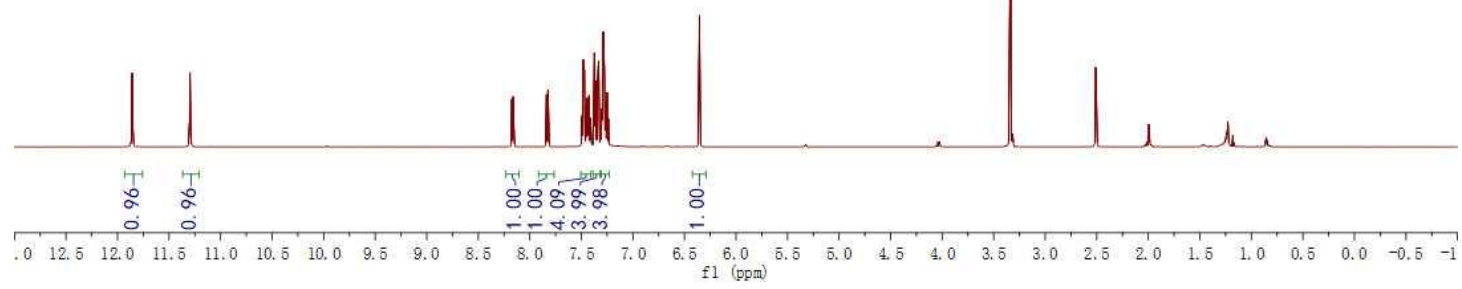

${ }^{13}$ C NMR (125 MHz, DMSO-d6)
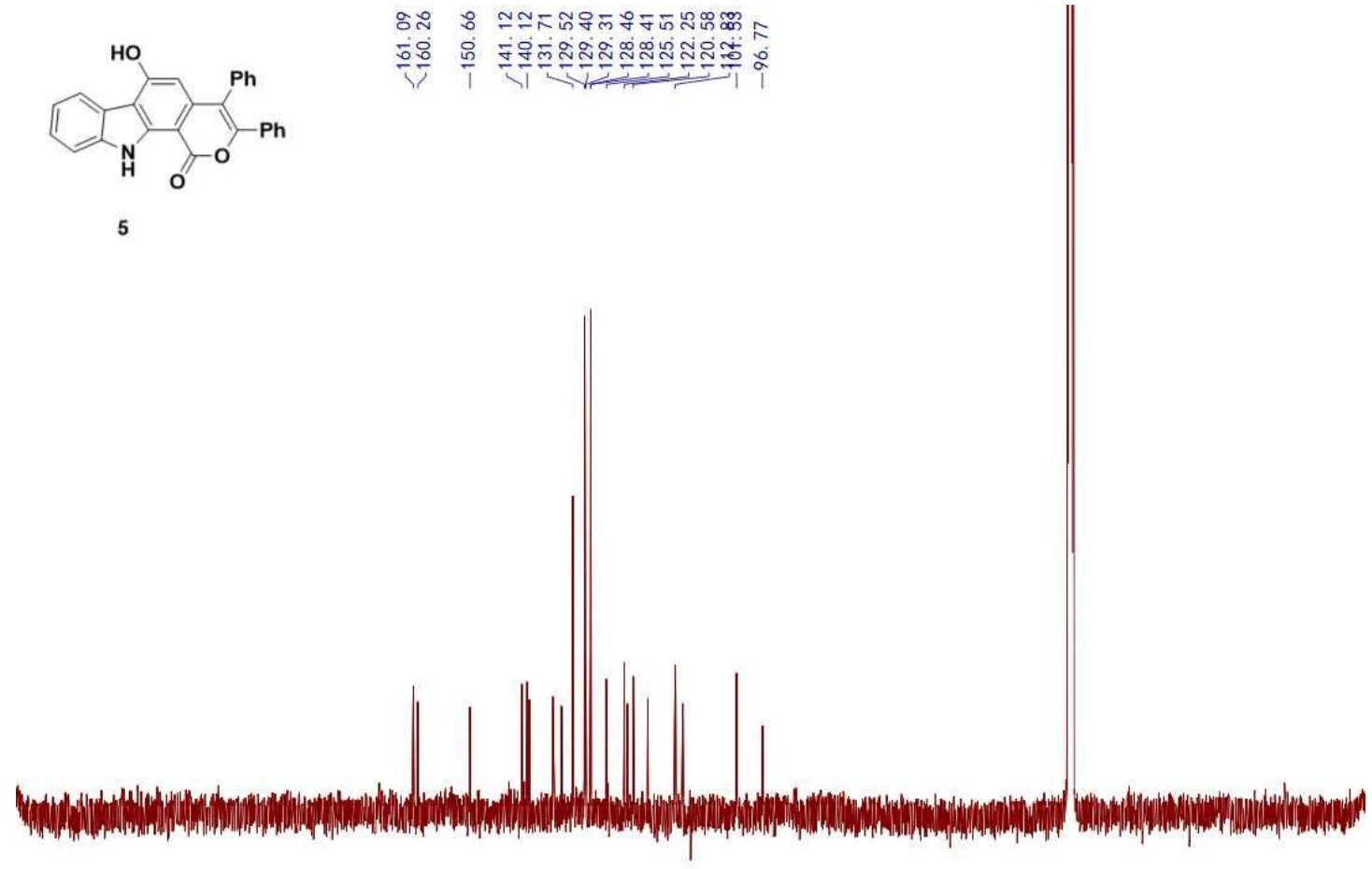

$\begin{array}{llllllllllll}230 & 220 & 210 & 200 & 190 & 180 & 170 & 160 & 150 & 140 & 130 & 120 \\ \mathrm{fl} 1(\mathrm{ppm}) & 100\end{array}$ 
${ }^{1}$ H NMR (500 MHz, DMSO-d6)
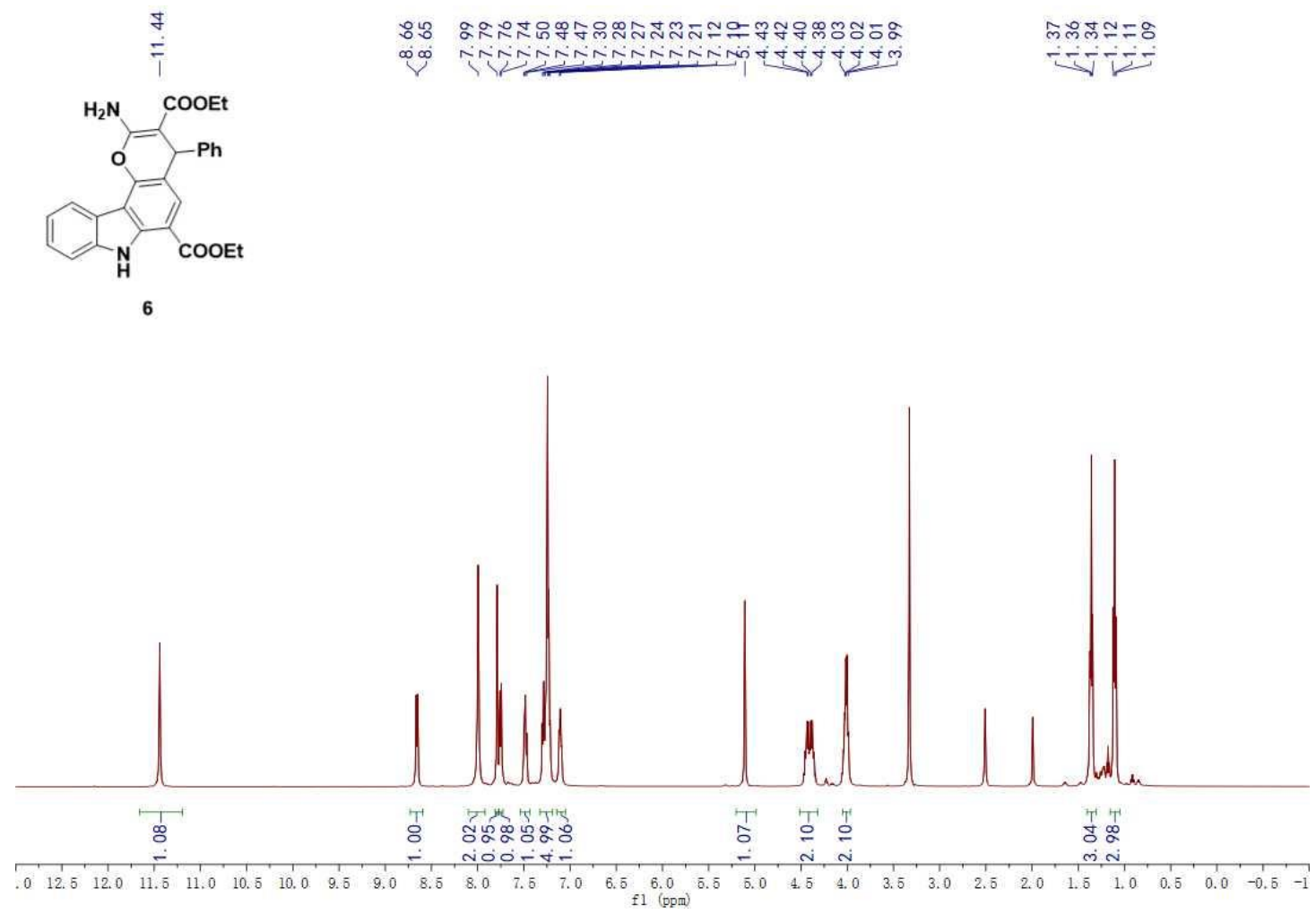

${ }^{13}$ C NMR (125 MHz, DMSO-d6)
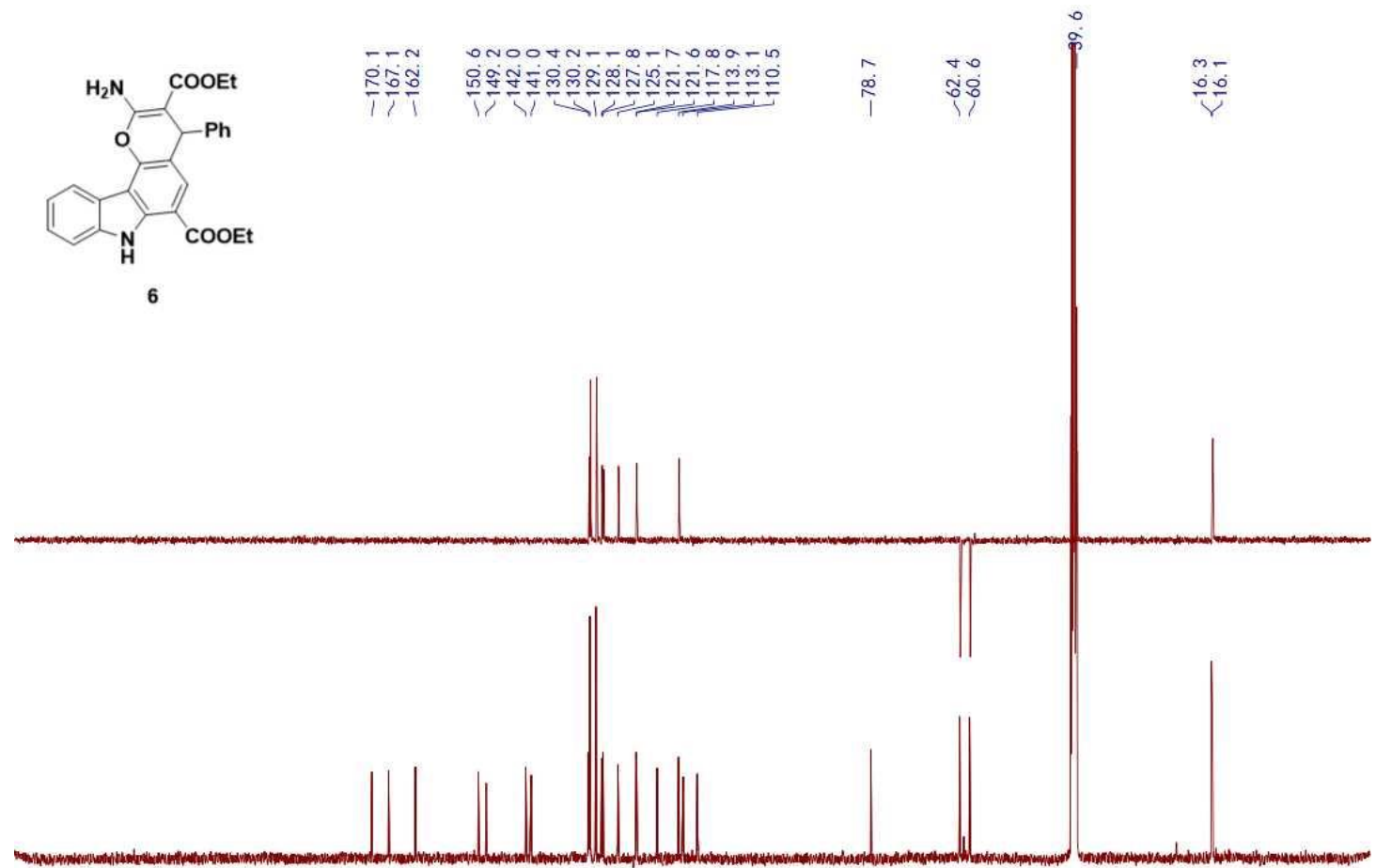

$\begin{array}{lllllllllllllllllllllllll}230 & 220 & 210 & 200 & 190 & 180 & 170 & 160 & 150 & 140 & 130 & 120 & 110 & 100 & 90 & 80 & 70 & 60 & 50 & 40 & 30 & 20 & 10 & 0 & -10\end{array}$ 